Preprint typeset in JHEP style. - HYPER VERSION

hep-th/0203048

\title{
Microscopic Formulation of Black Holes in String Theory
}

\author{
by Justin R. David ${ }^{a}$, Gautam Mandal ${ }^{b}$ and Spenta R. Wadia ${ }^{b}$ \\ ${ }^{a}$ Department of physics, \\ University of Californina, Santa Barbara, CA 93106, USA. \\ ${ }^{b}$ Tata Institute of Fundamental Research, \\ Homi Bhabha Road, Mumbai 400 005, India. \\ E-mail: justin@vulcan.physics.ucsb.edu, mandal@theory.tifr.res.in, \\ wadia@theory.tifr.res.in
}

\begin{abstract}
In this Report we review the microscopic formulation of the five dimensional black hole of type IIB string theory in terms of the D1-D5 brane system. The emphasis here is more on the brane dynamics than on supergravity solutions. We show how the low energy brane dynamics, combined with crucial inputs from AdS/CFT correspondence, leads to a derivation of black hole thermodynamics and the rate of Hawking radiation. Our approach requires a detailed exposition of the gauge theory and conformal field theory of the D1-D5 system. We also discuss some applications of the AdS/CFT correspondence in the context of black hole formation in three dimensions by thermal transition and by collision of point particles.
\end{abstract}

KEYWORDs: Black holes, String theory, D-branes, Gauge theory. 


\section{Contents}

1. Introduction 4

1.1 Quantum theory and general relativity

1.2 Black holes and the information puzzle 5

1.3 The string theory framework for black holes 9

1.4 Plan of this Report 11

2. Construction of classical solutions 18

2.1 Classical solutions of M-theory 18

2.1.1 The 2-brane solution of M-theory: M2 19

2.1.2 Intersecting M2-branes 22

2.2 The $6 \mathrm{D}$ black string solution of IIB on $T^{4}$

2.3 The extremal 5D black hole solution 25

2.4 Non-extremal five-dimensional black hole 27

2.4 .1 Geometry 29

2.4 .2 Hawking temperature and Bekenstein-Hawking entropy 29

2.4 .3 Comments on brane-antibrane and other non-BPS solutions 30

2.5 Supergravity Solution with Non-zero vev of $B_{N S}$

2.5.1 Asymptotically Flat Geometry 32

2.5 .2 Near Horizon Geometry 32

2.6 Near-horizon limit and $A d S_{3} \times S^{3}$

2.6.1 The three-dimensional anti de Sitter space or $\mathrm{AdS}_{3}$

2.6.2 The BTZ black hole 35

2.6 .3 The two-dimensional black hole 37

3. Semi-classical derivation of Hawking radiation 39

3.1 Minimal scalar 41

3.1.1 Absorption Cross-section: 43

3.1.2 Hawking radiation: 44

3.1 .3 Importance of near-horizon physics 44

3.2 Fixed scalars 45

4. The microscopic modeling of black hole and gauge theory of the D1-D5 system

4.1 The D1-D5 System and the $\mathcal{N}=4, U\left(Q_{1}\right) \times U\left(Q_{5}\right)$ gauge theory in 2-dimensions 46

4.2 The Potential Terms 50

4.3 D-Flatness Equations and the Moduli Space 51 
4.4 The Bound State in the Higgs Phase 52

4.5 The Conformally Invariant Limit of the Gauge Theory 54

4.6 Quick derivation of entropy and temperatures from CFT 54

4.7 D1 branes as solitonic strings of the D5 gauge theory 56

5. The SCFT on the orbifold $\mathcal{M}$

5.1 The $\mathcal{N}=4$ superconformal algebra 59

5.2 Free field realization of $\mathcal{N}=(4,4)$ SCFT on the orbifold $\mathcal{M}$

5.3 The $S O(4)$ algebra 60

5.4 The supergroup $S U(1,1 \mid 2)$

5.5 Short multiplets of $S U(1,1 \mid 2)$

5.6 The resolutions of the symmetric product 63

5.6.1 The untwisted sector 64

$5.6 .2 Z_{2}$ twists. 65

5.6 .3 Higher twists 67

5.7 The chiral primaries of the $\mathcal{N}=(4,4)$ SCFT on $\mathcal{M}$

5.7.1 The k-cycle twist operator 68

5.7.2 The complete set of chiral primaries 70

5.8 Short multiplets of $\mathcal{N}=(4,4)$ SCFT on $\mathcal{M}$

5.9 Stringy exclusion principle 73

6. Near horizon supergravity and SCFT 74

6.1 Classification of the supergravity modes 74

6.2 The supergravity moduli 77

$6.3 \mathrm{AdS}_{3} / \mathrm{CFT}_{2}$ correspondence 78

6.4 Supergravity moduli and the marginal operators 79

7. Location of the symmetric product 81

7.1 Dynamics of the decay of the D1-D5 system from gravity 84

7.2 The linear sigma model description of $R^{4} / Z_{2}$

7.3 The gauge theory relevant for the decay of the D1-D5 system 88

7.4 Dynamics of the decay of the D1-D5 system from gauge theory 93

7.5 The symmetric product 93

8. The microscopic derivation of Hawking radiation 95

8.1 Near horizon limit and Fermion boundary conditions 96

8.2 The black hole state 96

8.3 The coupling with the bulk fields for the D1-D5 black hole 99

8.4 Determination of the strength of the coupling $\mu$

8.4.1 Evaluation of the tree-level vertices in supergravity 101

8.4 .2 Two-point function 103

8.5 Absorption cross-section as thermal Green's function 104 
8.6 Absorption cross-section of minimal scalars from the D1-D5 SCFT 106 8.6.1 Absorption cross-section for the blow up modes 107

8.7 Fixed scalars 107

8.8 Intermediate Scalars 109

9. Non-renormalization theorems

9.1 The spectrum of short multiplets

9.2 Entropy and area 111

9.3 Hawking radiation 112

9.3.1 Independence of Hawking radiation calculation on moduli: Supergravity 112

9.3.2 Independence of Hawking radiation on moduli:SCFT 113

10. Strings in $A d S_{3}$

10.1 The S-dual of the D1-D5 system 118

10.2 String propagation on $A d S_{3}$

10.3 Spectrum of strings on $A d S_{3}$

10.4 Strings on Euclidean $A d S_{3}$

10.4 .1 The long string worldsheet algebra

10.5 Strings on the thermal $A d S_{3}$

11. Applications of $\mathrm{AdS}_{3}-\mathrm{CFT}_{2}$ duality 128

11.1 Hawking-Page transition in $\mathrm{AdS}_{3}$

11.2 Conical defects and particles in $A d S_{3}$

11.2.1 Black hole creation by particle collision 138

12. Concluding remarks and open problems 141

A. Euclidean derivation of Hawking temperature 144

B. A heuristic motivation for Rules1 and 2 in Section (2.4) 146

C. Coordinate systems for $\mathrm{AdS}_{3}$ and related spaces 148

C.1 $\mathrm{AdS}_{3}$

C.2 BTZ black hole 149

C.3 Conical spaces 150

C.4 Euclidean sections and Thermal Physics 151 


\section{Introduction}

\subsection{Quantum theory and general relativity}

Quantum theory and the general theory of relativity form the basis of modern physics. However, these two theories seem to be fundamentally incompatible. Quantizing general relativity leads to a number of basic problems:

1. Ultraviolet divergences render general relativity ill-defined as a quantum theory (see, e.g. S. Weinberg in [1]). This specifically means that if we perform a perturbation expansion around flat Minkowski space-time (which is a good first approximation to our world) then to subtract infinities from the divergent diagrams we have to add an infinite number of counterterms to the Einstein-Hilbert action with coefficients that are proportional to appropriate powers of the ultraviolet cutoff.

There is good reason to believe that string theory [2, 3] solves this ultraviolet problem because the extended nature of string interactions have an inherent ultraviolet cutoff given by the fundamental string length $\sqrt{\alpha^{\prime}}$. Furthermore, for length scales much larger than the string length the Einstein-Hilbert action emerges [4, [5 as a low energy effective action from string theory, with Newton's constant (for type II strings in ten dimensions) given by,

$$
G_{N}^{(10)}=8 \pi^{6} g_{s}^{2} \alpha^{\prime 4}
$$

where $g_{s}$ is the string coupling.

2. There are many singular classical solutions of general relativity (for standard textbooks on classical general relativity, see, e.g., [6, 7, 8, 9, 10]), including the Schwarzschild black hole and the Big Bang model of cosmology. Black holes and their higher dimensional analogues (black branes) also appear as solutions of low energy string theory. A quantum theory of gravity must (a) present an understanding regarding which of these singular geometries can arise from a well defined quantum mechanics in an appropriate limit, and (b) formulate such a quantum mechanics where possible. String theory has been able to "resolve" a class of singularities in this way, but a complete understanding of the issue of singularities is still lacking (see [11, 12, 13, 14, 15] for a partial list of related papers; 15 contains a review and a comprehensive list of references).

3. While the above problems are related to the high energy (short distance) behaviour of general relativity, there exists another basic problem when we quantize matter fields in the presence of a black hole, which does not ostensibly depend on high energy processes. This problem is called the information 
puzzle ([16, 17], for early reviews see, e.g., [1, 18, 19]). In the following we shall explain the issue in some detail and subsequently summarize the attempts within string theory to resolve the puzzle in a certain class of black holes.

Besides being a long-lasting problem of general relativity, this is an important problem for string theory for the following reason. String theory has been proposed as a theory that describes all elementary particles and their interactions. Presently the theory is not in the stage of development where it can provide quantitative predictions in particle physics. However if string theory can resolve some logical problem that arises in the applications of standard quantum field theory to general relativity, then it is a step forward for string theory.

4. Finally, any quantum theory of gravity should lead to an understanding of the problem of the cosmological constant (for reviews see, e.g. [20], 221], 22]).

\subsection{Black holes and the information puzzle}

Let us briefly review some general properties of black holes [6, 7, 8, 9, 10]. Black holes are objects which result as end points of gravitational collapse of matter. For an object of mass greater than roughly three solar masses (see, e.g. [23]), the gravitational force overcomes all other forces and the matter generically collapses into a black hole (in some exceptional cases a naked singularity might result). This would suggest that to specify a black hole it is necessary to give in detail the initial conditions of the collapse. As we will see below a black hole is completely specified by a few parameters only.

To introduce various concepts related to black holes we will discuss two examples of black holes. First, let us consider the Schwarzschild black hole in $3+1$ dimensions. It is a time independent, spherically symmetric solution of Einstein gravity without matter. The metric is given by

$$
d s^{2}=-\left(1-\frac{2 G_{N} M}{r}\right) d t^{2}+\left(1-\frac{2 G_{N} M}{r}\right)^{-1} d r^{2}+r^{2} d \Omega^{2}
$$

where $G_{N}$ is the Newton's constant ${ }^{1}$ and $d \Omega^{2}=\left(d \theta^{2}+\theta^{2} d \phi^{2}\right)$ is the metric on $S^{2}$. We have chosen units so that the velocity of light is $c=1$. The surface $r=2 G_{N} M$ is called the event horizon. It is a coordinate singularity $\left(g_{r r}=\infty\right)$ but not a curvature singularity (e.g., Ricci scalar is finite here). Light-like geodesics and time-like geodesics starting "inside" the event horizon $\left(r<2 G_{N} M\right)$ end up at $r=0$ (the curvature singularity) in a finite proper time. This means that

\footnotetext{
${ }^{1}$ We will use the notation $G_{N}^{(D)}$ or $G_{N}^{D}$ for Newton's constant in $D$ spacetime dimensions, reserving the default $G_{N}$ for $D=4$.
} 
classically the black hole is truly black, it cannot emit anything. Note that the solution is completely specified by only one parameter $M$, which coincides with the ADM mass (see (2.12)) of the black hole.

Next we consider the Reissner-Nördstrom (RN) black hole. It is a time independent, spherically symmetric solution of Einstein gravity coupled to the electromagnetic field. The solution is given by the following backgrounds:

$$
\begin{aligned}
d s^{2} & =-\left(1-\frac{2 G_{N} M}{r}+\frac{G_{N} Q^{2}}{r^{2}}\right) d t^{2}+\left(1-\frac{2 G_{N} M}{r}+\frac{G_{N} Q^{2}}{r^{2}}\right)^{-1} d r^{2}+r^{2} d \Omega^{2} \\
A_{0} & =\frac{Q}{r}, \quad A_{i}=0, i=1,2,3 .
\end{aligned}
$$

where $A_{0}$ is the time component of the vector potential. This solution carries a charge $Q$ (the Schwarzschild black hole, Eqn. (1.2), corresponds to the special case $Q=0)$. There are two coordinate singularities $\left(g_{r r}=\infty\right)$ at $r=r_{+}$(outer horizon) and $r=r_{-}$(inner horizon)

$$
r_{ \pm}=G_{N} M \pm \sqrt{\left(G_{N} M\right)^{2}-G_{N} Q^{2}}
$$

The event horizon coincides with the outer horizon $r=r_{+}$. When $M=|Q| / \sqrt{G_{N}}$, $r_{+}$coincides with $r_{-}$. Such a black hole is called an extremal black hole. Note that the black hole (1.3) is completely specified by its mass $M$ and the charge $Q$.

The D1-D5 black hole, which will be the main subject of this report, is similar to the RN black hole; it is charged under Ramond-Ramond fields in type IIB string theory and has the same causal structure, equivalently the same Penrose diagram (see Section 2.4.1), as the RN black hole.

The point $r=0$ is a curvature singularity for both the Schwarzschild solution (1.2) and the Reissner-Nördstrom solution (1.3). The singularity is "clothed" by the event horizon in the physical range of parameters $(M>0$ for Schwarzschild, $M>|Q| / \sqrt{G_{N}}$ for RN). The ranges of mass: $M<0$ for Schwarzschild, $M<$ $|Q| / \sqrt{G_{N}}$ for RN, are unphysical because these result in a naked singularity (see, e.g. [11). The limiting case for the RN black hole, $M=|Q| / \sqrt{G_{N}}$, is called an extremal black hole, as already mentioned above.

In general, collapsing matter results in black holes which are completely specified by the mass $M$, the $U(1)$ charges $Q_{i}$ and the angular momentum $J$. This is called the no hair theorem (see, e.g., [24, 25, 26, 27] for a review). Whatever other information (for example, multipole moments) present decays exponentially fast with a characteristic time $\tau=r_{h} / c$, during the collapse. $\left(r_{h}\right.$ is the radius of the horizon and $c$ is the speed of light). Thus, all detailed information carried by the collapsing matter is completely lost.

The other beautiful result of classical general relativity is the so called area law. It says that [10] the area of the horizon of a black hole cannot decrease with 
time, and if two (or more) black holes (of areas $A_{1}, A_{2}, \ldots$ ) merge to form a single black hole, the area $A$ of its horizon will satisfy

$$
A \geq A_{1}+A_{2}+\ldots
$$

This general result is easy to verify for the Schwarzschild and Reissner-Nördstrom black holes that we have discussed above.

Bekenstein argued that unless a black hole had entropy, an infalling hot body would lead to a violation of the second law of thermodynamics because the entropy of the hot body will be lost once it is absorbed by the black hole, thus causing entropy to decrease. He postulated that the entropy of a black hole is proportional to the area of the event horizon, the constant of proportionality being universal for all black holes. The area law now have natural thermodynamic interpretations and the macroscopic observables allowed by the no hair theorems play the role of macroscopic thermodynamic variables. In the classical theory, however, there is no notion of the absolute entropy of a system. Such a concept, as we shall see now, requires quantum theory.

In his pioneering work in the seventies, Hawking [16] quantized matter fields in the background geometry of a black hole. He found by that the Schwarzschild black hole is not truly black. A semi-classical calculation showed that it emits radiation with the spectrum of a black body at a temperature $\mathrm{T}$ given by

$$
T_{H}=\frac{\hbar}{8 \pi G_{N} M}
$$

The quantum nature of this effect is clearly evident from the fact that the temperature is proportional to $\hbar$. For the Reissner-Nördstrom black hole the temperature of Hawking radiation is

$$
T_{H}=\frac{\left(r_{+}-r_{-}\right) \hbar}{4 \pi r_{+}^{2}}
$$

where $r_{ \pm}$are defined in (1.4) as functions of $Q, M$. A brief derivation of the temperature formulae, using the Euclidean approach [28] is presented in Appendix A. One notes that the extremal Reissner-Nördstrom black hole has $T_{H}=0$ and does not Hawking radiate.

In general the Hawking temperature turns out to be a function of mass, the charge(s) and the angular momentum alone. Thus even semi-classical effects do not provide further information of the black hole. The works in the seventies culminated in the following laws of black holes [29, 30, 31, 32] which are analogous to the laws of thermodynamics.

1. First Law: Two neighbouring equilibrium states of a black hole, of mass $M$, charges $Q_{i}$ and angular momentum $J$, are related by

$$
d M=T_{H} d\left[\frac{A}{4 G_{N} \hbar}\right]+\Phi_{i} d Q_{i}+\Omega d J
$$


where $A$ is the area of the event horizon, $\Phi$ the electric surface potential and $\Omega$ the angular velocity. For the special case of the Reissner-Nördstrom black hole the first law reduces to

$$
d M=T_{H} d\left[\frac{A}{4 G_{N} \hbar}\right]+\Phi d Q .
$$

where $\Phi=Q / r_{+}$. This is explicitly verified in appendix A, Eqn. (A.11).

2. Second Law: Black holes have entropy $S$ given by

$$
\begin{aligned}
S & =\frac{A}{4 G_{N} \hbar}=\frac{1}{4} \frac{A}{A_{P l}} \\
A_{P l} & =\frac{G_{N} \hbar}{c^{3}}
\end{aligned}
$$

where we have reinstated in the last equation the speed of light $c$. The generalized second law says that the sum of the entropy of the black hole and the surroundings never decreases (this is a generalized form of (1.5)).

Formula (1.10), called the Bekenstein-Hawking entropy formula, is very important because it provides a counting of the effective number of degrees of freedom of a black hole which any theory of quantum gravity must reproduce. We note that $A_{P l}$ in (11.11) is a basic unit of area (Planck unit), involving all three fundamental constants $\left(A_{P l}=2.61 \times 10^{-66} \mathrm{~cm}^{2}\right.$ in four dimensions $)$. The Bekenstein-Hawking formula simply states that the entropy of a black hole is a quarter of its horizon area measured in units of $A_{\mathrm{Pl}}$.

The entropy of the Schwarzschild black hole, according to (1.10) is

$$
S=4 \pi G_{N} M^{2}
$$

while that of the $\mathrm{RN}$ black hole is

$$
S=\frac{\pi r_{+}^{2}}{G_{N} \hbar}
$$

The Hawking radiation as calculated in semi-classical general relativity is a mixed state. It turns out to be difficult to calculate the correlations between the infalling matter and outgoing Hawking particles in the standard framework of general relativity. Such a calculation would require a good quantum theory of gravity where controlled approximations are possible [33].

If we accept the semi-classical result that black holes emit radiation that is exactly thermal then it leads to the information puzzle (匹16, 17, 1, 18, 19]):

Initially the matter that formed the black hole is in a pure quantum mechanical state. Here in principle we know all the quantum mechanical correlations between the degrees of freedom of the system. In case the black hole evaporates completely 
then the final state of the system is purely thermal and hence it is a mixed state. This evolution of a pure state to a mixed state is in conflict with the standard laws of quantum mechanics which involve unitary time evolution which sends pure states to pure states.

Hence it would appear that we have to modify quantum mechanics, as was advocated by Hawking [17]. However, in the following we shall argue that if we replace the paradigm of quantum field theory by that of string theory (Section 1.3), we are able to retain quantum mechanics and resolve the information puzzle (for a certain class of black holes) by discovering the microscopic degrees of freedom of the black hole.

It is well worth pointing out that the existence of black holes in nature (for which there is mounting evidence [23]) compels us to resolve the conundrums that black holes present. One can perhaps take recourse to the fact that for a black hole whose mass is a few solar masses the Hawking temperature is very tiny $\left(\sim 10^{-8}\right.$ degree Kelvin), and not of any observable consequence. However the logical problem that we have described above cannot be wished away and its resolution makes a definitive case for the string paradigm as a correct framework for fundamental physics as opposed to standard local quantum field theory.

This assertion implicitly assumes that in string theory there exists a controlled calculational scheme to calculate the properties of black holes. Fortunately there does exist a class of black holes in type IIB string theory compactified on a 4manifold ( $T^{4}$ or $\left.K_{3}\right)$ which has sufficient supersymmetry to enable a precise calculation of low energy processes of this class of black holes. This aspect is the main focus of this review.

\subsection{The string theory framework for black holes}

We now briefly describe the conceptual framework of black hole thermodynamics in string theory. A black hole of a given mass $M$, charges $Q_{i}$ and angular momentum $J$ is defined by a density matrix (see also (8.2)):

$$
\rho=\frac{1}{\Omega} \sum_{i \in \mathcal{S}}|i\rangle\langle i|
$$

where $|i\rangle$ is a microstate which can be any of a set $\mathcal{S}$ of states (microcanonical ensemble) all of which are characterized by the above mentioned mass, charges and angular momentum.

A definition like (1.14) is of course standard in quantum statistical mechanics, where a system with a large number of degrees of freedom is described by a density matrix to derive a thermodynamic description. Using (1.14) thermodynamic quantities like the temperature, entropy and the rates of Hawking radiation can be derived for a black hole in string theory (see Section 8 for details). In particular 
the Bekenstein-Hawking formula is derived from Boltzmann's law:

$$
S=\ln \Omega
$$

where $\Omega$ is the number of microstates of the system. We are using units so that the Boltzmann constant is unity.

Given this we can calculate formulas of black hole thermodynamics just like we calculate the thermodynamic properties of macroscopic objects using standard methods of statistical mechanics. Here the quantum correlations that existed in the initial state of the system are in principle all present and are only erased by our procedure of defining the black hole state in terms of a density matrix. In this way one can account for not only the entropy of the system which is a counting problem but also the rate of Hawking radiation which depends on interactions.

Let us recall the treatment of radiation coming from a star, or a lump of hot coal. The 'thermal' description of the radiation coming is the result of averaging over a large number of quantum states of the coal. In principle by making detailed measurements on the wave function of the emitted radiation we can infer the precise quantum state of the emitting body. For black holes the reasoning is similar.

Hence in the string theory formulation the black hole can exist as a pure state: one among the highly degenerate set of states that are characterized by a small number of parameters. Let us also note that in Hawking's semi-classical analysis, which uses quantum field theory in a given black-hole space-time, there is no possibility of a microscopic construction of the black hole wave functions. To repeat, in string theory Hawking radiation is not thermal and in principle we can reconstruct the initial state of the system from the final state, which therefore resolves the information paradox.

We shall see that in the case of the five-dimensional black hole of type IIB string theory it is possible to construct a precise microscopic description of black hole thermodynamics. We now summarize the four basic ingredients we need to describe and calculate Hawking radiation for near extremal black holes which have low Hawking temperature:

1. The microscopic constituents of the black hole. In the case of the 5 -dimensional black hole of type IIB string theory the microscopic modeling is in terms of a system of D1-D5 branes wrapped on $S^{1} \times M_{4}$, where $M_{4}$ is a 4-dim. compact manifold, which can be either $T^{4}$ or $K_{3}$. In this report we will mainly focus on $T^{4}$.

2. The spectrum of the low energy degrees of freedom of the bound state of the D1-D5 system. These are derived at weak coupling and we need to know if the spectrum survives at strong coupling.

3. The coupling of the low energy degrees of freedom to supergravity modes. 
4. The description of the black hole as a density matrix. This implies expressions for decay and absorption probabilities which are related to S-matrix elements between initial and final states of the black hole.

To understand the microscopic calculation of Hawking rate in a nutshell, consider a black hole of mass $M$ and charge $Q$, described by a microcanonical ensemble $\mathcal{S}$. Consider the process of absorption of some particles by the black hole which changes the mass and charge to $M^{\prime}, Q^{\prime}$ (corresponding to another microcanonical ensemble, say $\mathcal{S}^{\prime}$ ). Let $\Omega\left(\Omega^{\prime}\right)$ be the total number of states in $\mathcal{S}$ (resp. $\mathcal{S}^{\prime}$ ). The absorption probability from a state $|i\rangle \in \mathcal{S}$ to a state $|f\rangle \in \mathcal{S}^{\prime}$ is given by

$$
P_{\text {abs }}(i \rightarrow f)=\frac{1}{\Omega} \sum_{i, f}|\langle f|S| i\rangle|^{2}
$$

where, by definition, we sum over the final states and average over the initial states. Similarly, the decay probability from a state $\mid i>\in \mathcal{S}^{\prime}$ to a state $\mid f>\in \mathcal{S}$ is given by

$$
P_{\text {decay }}(i \rightarrow f)=\frac{1}{\Omega^{\prime}} \sum_{i, f}|\langle f|S| i\rangle|^{2}
$$

Point (3) above allows us to calculate the matrix element $\langle f|S| i\rangle$ in string theory, thus leading to a calculation of absorption cross-section and Hawking rate. We will elaborate on this in detail in Section 8.

One of the important issues in this subject is that the string theory ingredients (points (1) and (2) above) are usually known in the case when the effective open string coupling is small. In this case the Schwarzschild radius $R_{s c h}$ of the black hole is smaller than the string length $l_{s}$ and we have a controlled definition of a string state. As the coupling is increased we go over to the supergravity description where $R_{s c h} \gg l_{s}$ and we have a black hole. Now it is an issue of dynamics whether the spectrum of the theory undergoes a drastic change or not, determining whether the description of states in weak coupling, which enabled a thermodynamic description, remains valid or not. In the model we will consider we will see that the description of the weak coupling effective Lagrangian goes over to strong coupling because of supersymmetry. It is an outstanding challenge to understand this problem when the weak coupling theory has little or no supersymmetry [34, 35, 36, 37]. We will briefly touch upon this issue in Section 11.

\subsection{Plan of this Report}

The plan of the rest of this Report is as follows.

In Section 2, we discuss in some detail the construction of the five-dimensional black hole solution in type IIB supergravity. We provide a brief background of how to construct BPS solutions in M-theory from first principles by explicitly

solving Killing spinor equations; we focus on the example of the M2 brane and 
its intersections. We then construct the D1-D5 black string solution by dualizing $\mathrm{M} 2 \perp \mathrm{M} 2$, and the extremal (BPS) D1-D5 black hole by dualizing M2 $\perp \mathrm{M} 2 \perp \mathrm{M} 2$. We then describe an algorithm of how to generate non-BPS solutions from BPS ones; we provide a motivation for this algorithm in Appendix B. We compute the Bekenstein-Hawking entropy and the Hawking temperature of these black holes. We next discuss how to generate non-zero $B_{\mathrm{NS}}$ in the D1-D5 system by exact dualities in supergravity. In the absence of the $B_{\mathrm{NS}}$ vev, the D1-D5 system is marginally bound and can fragment into clusters of smaller D1-D5 systems. We show, by constructing the exact supergravity solution with non-zero $B_{\mathrm{NS}}$, that the BPS mass formula becomes non-linear in the masses of the D1 and D5 branes and that a binding energy is generated. The picture of the bound state thus obtained is compared with gauge theory and conformal field theory in Sections 4 and 7. Next, we describe the appearance of $\mathrm{AdS}_{3}$ and BTZ black holes as the "near-horizon limit" of the D1-D5 string and the D1-D5 black hole, respectively; this discussion is used as background material (together with Appendix C) for the discussions of AdS/CFT in Sections 6 and 11. In the context of the near-horizon physics we also discuss a connection between the pure five-brane system and the two-dimensional black hole.

In Section 3, we describe the semiclassical derivation of absorption cross-section (also called graybody factor) and the rate of Hawking radiation for the nearextremal five-dimensional black hole constructed in Section 2. We describe various fluctuations around the black hole solutions. We specifically focus on (a) minimal scalars which couple only to the five-dimensional Einstein-frame metric, and (b) fixed scalars which couple also to other background fields like the Ramond-Ramond fluxes. We derive case (a) in more detail in which we show that the computation of the graybody factor and the Hawking rate essentially depends only on the nearhorizon limit. Both cases (a) and (b) are compared with the D1-D5 conformal field theory calculations in Section 8 where we find exact agreement with the results of Section 3. The agreement involves new first principles calculations in the D-brane conformal field theory which involve significant conceptual departures from the phenomenological approach adopted in the early calculations (see [38, 39, 40] for the original papers and [41] for a review). We mention the new points in detail in Section 8 (esp. Secs 8.6.1, 8.7). The results in Section 3 and Section 8 have a priori different regions of validity; a comparison therefore involves an extrapolation which is made possible by non-renormalization theorems discussed in Section 9.

In Section 4, we discuss the gauge theory for the D1-D5 system. This system is closely related to the five-dimensional black hole solution. The D1-D5 system, as discussed in Section 2, consists of $Q_{1}$ D1 branes and $Q_{5}$ D5 branes wrapped on a $T^{4}$ in type IIB theory. It is a black string in six dimensions. The five-dimensional black hole solution is obtained by wrapping the black string on a circle and introducing Kaluza-Klein momenta along this direction. For the purpose of understanding 
Hawking radiation from the five-dimensional black hole it is sufficient to study the low energy effective theory of the D1-D5 system. The low energy theory of the D1-D5 system is a $1+1$ dimensional supersymmetric gauge theory with gauge group $U\left(Q_{1}\right) \times U\left(Q_{5}\right)$. It has 8 supersymmetries. The matter content of this theory consists of hypermultiplets transforming as bi-fundamentals of $U\left(Q_{1}\right) \times U\left(Q_{5}\right)$. We review in detail the field content of this theory and its symmetries. The bound state of the D1 and D5 branes is described by the Higgs branch of this theory. We show the existence of this bound state when the Fayet-Iliopoulos terms are non-zero. The Higgs branch of the D1-D5 system flows in the infrared to a certain $\mathcal{N}=(4,4)$ superconformal field theory (SCFT). A more detailed understanding of this conformal field theory can be obtained by thinking of the bound D1 branes as solitonic strings of the D5 brane theory. From this point of view the target space of the SCFT is the moduli space of $Q_{1}$ instantons of a $U\left(Q_{5}\right)$ gauge theory on $T^{4}$. This moduli space is known to be a resolution of the orbifold $(\tilde{T})^{Q_{1} Q_{5}} / S\left(Q_{1} Q_{5}\right)$ which we denote by $\mathcal{M}$. $S\left(Q_{1} Q_{5}\right)$ stands for the symmetric permutation group of $Q_{1} Q_{5}$ elements. The torus $\tilde{T}^{4}$ can be distinct from the compactification torus $T^{4}$. We review the evidence for this result. The mere fact that the low energy theory is a (super)conformal field theory with a known central charge, is used in Section 4.6 to provide a short-hand derivation of the Bekenstein-Hawking entropy and Hawking temperature. The complete derivation, including that of the graybody factor and Hawking rate, is however possible only in Section 8 after we have discussed in detail the precise SCFT in Section 5,the couplings to the bulk fields in Section 6 and the location of the SCFT used in the moduli space of the $\mathcal{M}=(4,4) \mathrm{SCFT}$ on $\mathcal{M}$. It turns out the supergravity calculation and the SCFT are at different points in the moduli space and the reason they agree is due to the non-renormalization theorems discussed in section 9 .

In Section 5 we formulate the $\mathrm{SCFT}$ on $\mathcal{M}$, we will discuss in detail a realization of this orbifold SCFT as a free field theory with identifications. The symmetries of this SCFT including a new $S O(4)$ algebra will also be discussed. As the SCFT relevant for the D1-D5 system is a resolution of the SCFT on the orbifold $\mathcal{M}$, we construct all the marginal operators of this SCFT including operators which correspond to blowing up modes. We classify the marginal operators according to the short multiplets of the global part of the $\mathcal{N}=(4,4)$ superalgebra and the new $S O(4)$ algebra. We then explicitly construct all the short multiplets of the SCFT on $\mathcal{M}$ and classify them according to the global subgroup of the $\mathcal{N}=(4,4)$ superconformal algebra. These short multiplets are shown to be in one to one correspondence with the spectrum of short-multiplets of supergravity in the near horizon geometry of the D1-D5 system in section 6.

Now that we have discussed the microscopic degrees of freedom in section 6 we take the next step towards the microscopic understanding of Hawking radiation. We find the precise coupling of the fields of the supergravity to the microscopic 
SCFT. This is given by a specific SCFT operator $\mathcal{O}(z, \bar{z})$, which couples to the supergravity field $\phi$ in the form of an interaction.

$$
S_{\mathrm{int}}=\mu \int d^{2} z \phi(z, \bar{z}) \mathcal{O}(z, \bar{z})
$$

where $\mu$ is the strength of the coupling. As the $\mathcal{N}=(4,4)$ SCFT on $\mathcal{M}$ is an "effective" theory of the D1-D5 system, it is difficult to fix the coupling of this theory to the supergravity fields using ab-initio methods. We fix the operator in the SCFT corresponding to the supergravity field using the method of symmetries. The near horizon limit of the D1-D5 system exhibits enhanced symmetries. This is a special case of the AdS/CFT correspondence. As we have seen in section 2 the near horizon geometry of the D1-D5 system reduces to that of $A d S_{3} \times S^{3} \times T^{4}$. The AdS/CFT correspondence [42] states that string theory on $A d S_{3} \times S^{3} \times T^{4}$ is dual to the $1+1$ dimensional SCFT describing the Higgs branch of the D1-D5 system on $T^{4}$. For large $Q_{1} Q_{5}$ the radius of $S^{3}$ is large in string units. The Kaluza-Klein modes on $T^{4}$ is of the order of string length. Thus type IIB string theory on $A d S_{3} \times S^{3} \times T^{4}$ passes over to 6 dimensional $(2,2)$ supergravity on $A d S_{3} \times S^{3}$. We will work in the supergravity limit. The evidence for this correspondence comes from symmetries. The isometries of $A d S_{3}$ correspond to the global part of the Virasoro group of the SCFT. The R-symmetry of the SCFT is identified with the isometry of the $S^{3}$. The number of supersymmetries of the bulk get enhanced to 16 from 8 . These correspond to the global supersymmetries of the $\mathcal{N}=(4,4)$ superalgebra of the SCFT. Thus the global part of the superalgebra of the SCFT is identified with the $A d S_{3} \times S^{3}$ supergroup, $S U(1,1 \mid 2) \times S U(1,1 \mid 2)$. Therefore a viable strategy to fix the coupling is to classify both the bulk fields and the SCFT operators according to the symmetries. The question then would be if this procedure can fix the SCFT operator required for analysing Hawking radiation. We will review the classification of the entire set of Kaluza-Klein modes of the six-dimensional supergravity on $S^{3}$ as short multiplets of $S U(1,1 \mid 2) \times S U(1,1 \mid 2)$ [43]. We use symmetries, including the new global $S O(4)$ algebra, to identify the marginal operators constructed in section 5 with their corresponding supergravity fields. The enables us to identify the operators corresponding to the minimal scalars. We also identify the quantum numbers of the fixed scalars and the intermediate scalars.

From section 5 and section 6 we see that there is a one to one correspondence between the moduli of the $\mathcal{N}=(4,4)$ SCFT on $\mathcal{M}$ and the moduli of supergravity. In section 7 we address the question where in this moduli space is the free field orbifold SCFT, which we use in our calculations, with respect to the supergravity solution. Let us first examine the supergravity side. In section 2 we constructed the supergravity solution with the self-dual Neveu-Schwarz $B$ field turned on. The self dual NS B-field is a moduli of the D1-D5 background. The mass formula for these solutions was nonlinear and had a binding energy for the break up of the D1-D5 
system into constituent D1 and D5 branes. This is unlike the case when no moduli is turned on. The mass formula in that case is linear and the system is marginally stable with respect to decay into constituent branes. We see in section 7 that the effective theory from supergravity governing the decay of the D1-D5 system is a Liouville theory. This is derived by using probe branes near the boundary of $A d S_{3}$. These branes are called long strings. We then derive this Liouville theory from the D1-D5 gauge theory. As we have seen in section 4, absence of a bound state implies that the Fayet-Iliopoulos and $\theta$ terms have to be set to zero. In section 7 we see that the effective theory near the origin of the Higgs branch (i.e. when these terms are small but non-zero) is the same Liouville theory as seen in supergravity. This Liouville theory is strongly coupled and singular at the origin of the Higgs branch. The free orbifold SCFT on $\mathcal{M}$, on the other hand, is regular and finite. Thus we conclude that the free orbifold theory must correspond, (a) in the gauge theory to a point where the Fayet-Iliopoulos and/or $\theta$ terms do not vanish, equivalently (b) in supergravity when some of the moduli are turned on which lead to non-zero binding energy, as mentioned above. More specifically we will see that the orbifold theory corresponds to a point where the $\theta$ term in the gauge theory of the D1-D5 system is turned on. Thus the simple supergravity solution of Sections 2.3 and 2.4 is at a different point in moduli space compared to the SCFT. In section 9 we describe why calculations done in the free orbifold SCFT can be valid at the point in moduli space which corresponds to the simple supergravity solution.

In Section 8, we derive the thermodynamics of the five-dimensional black hole from the microscopic viewpoint (SCFT). We use the free field orbifold $\mathcal{N}=(4,4)$ SCFT on $\mathcal{M}$ as the microscopic theory. The D1-D5 black hole is identified as an excited state with definite left and right conformal weights over the Ramond sector of this theory. Using Cardy's formula we get the asymptotic state counting for a conformal field theory in terms of its central charge and the level number. Use of the Cardy formula in the SCFT for the black hole state shows that the entropy evaluated from SCFT precisely matches with the entropy of D1-D5 black hole. We then discuss Hawking radiation of minimal scalars from the D1-D5 black hole. In order to do this we have to fix the strength of the coupling $\mu$ of the minimal scalar to its boundary operator in (1.18). We determine this by matching the two point functions evaluated in the SCFT and supergravity. It is only here we use the more quantitative version of the AdS/CFT correspondence. Once the coupling, and hence $S_{i n t}$ is determined, Hawking radiation/absorption cross-section from the microscopic SCFT can be derived as a purely quantum scattering process. We formulate the absorption cross-section calculation from the SCFT as an evaluation of the thermal Green's function of the operators $\mathcal{O}$ in (1.18). The absorption crosssection evaluated from SCFT agrees with the semi-classical calculation evaluated in supergravity in section 3. In Sections 8.6 .1 and 8.7 we point out the differences between the present SCFT results and those in the early works (reviewed in [41]) 
which, adopted a phenomenological approach for the D-brane degrees of freedom as well as the coupling to supergravity fields. The phenomenological method had a discrepancy for 4 minimal scalars (which correspond to blow-up modes in the SCFT) and fixed scalars from the semi-classical results of Section 3. In fact using the phenomenological Dirac-Born-Infeld action for the D-brane degrees of there is no accounting at all for the minimal scalars corresponding to the blow-up modes. We show that the first principles calculation presented in Section 8 remove these discrepancies. We also outline the absorption cross-section calculation for the intermediate scalars.

Though the $\mathcal{N}=(4,4)$ SCFT and the near horizon moduli of supergravity agree, we have seen in section 7 that the free field orbifold theory is at a different point in moduli space compared to the supergravity solution. In section 9 we see why calculations done using the free orbifold theory are valid and agree with that of supergravity. We show how the spectrum of short multiplets, the entropy and the calculation of Hawking radiation are independent of moduli both on the SCFT and supergravity side due to non-renormalization theorems. In discussing non-renormalization theorems for Hawking radiation we first examine the dilute gas approximations and the low energy approximation made in the semi-classical calculation in section 3 and show how they appear in the SCFT.

In section 10 we go beyond the supergravity approximation in the study of the near horizon geometry, $A d S_{3} \times S^{3} \times T^{4}$ and study string theory in this curved background to explore the AdS/CFT correspondence. Since string theory in RamondRamond backgrounds are difficult to study it is convenient to study the near horizon geometry of the S-dual to the D1-D5 system, the NS1-NS5 brane system. We review the spectrum of strings in this background and show that the long strings found in section 7 play an important role in the completion of the spectrum. We then review the formulation of string theory on Euclidean $A d S_{3}$ and derive the world sheet algebra of the long string. It is seen, as expected from S-duality, that the world sheet theory is a lioville theory which coincides with that of the single long D1-string near near the boundary of $A d S_{3}$ in section 7 . We then review the elvaluation of the partition function of a gas of strings in thermal $A d S_{3}$. From this partition function it can be verified that long strings are present in the spectrum as expected from the analysis of the spectrum with the Lorentzian signature metric.

In Section 11, we discuss some applications of AdS/CFT correspondence (introduced in Section 6). In the first part we discuss the thermal phase transition (Hawking-Page) in $\mathrm{AdS}_{3}$ and how to understand it in terms of the dual CFT picture. We show that the Euclidean partition of asymptotically $\mathrm{AdS}_{3}$ spaces can be evaluated in the leading semiclassical approximation as a sum over an SL(2,Z) family of saddle-point configurations, of which two members are $\mathrm{AdS}_{3}$ and the BTZ black hole. We discuss the issue of boundary conditions of this partition function (see also Appendix C) and relate it to the boundary conditions of the CFT par- 
tition function. We calculate the BTZ partition function in $\mathrm{AdS}_{3}$ supergravity as well as in the boundary SCFT and show that they agree. We show that the supergravity partition function is dominated at low energies by $\mathrm{AdS}_{3}$, and high energies by the Euclidean BTZ black hole; from the CFT viewpoint this phenomenon gets related to the fact that at low temperatures the NS sector dominates and high temperatures the Ramond sector dominates. The modular invariance of the boundary SCFT points gets related to a similar modular invariance in the three-dimensional supergravity. We next describe some new spaces which are also asymptotically $\mathrm{AdS}_{3}$; these are conical spaces which are created by static point particles. We discuss various ways of understanding them as solutions of string theory, the simplest being the case of $Z_{N}$ cones which are described as orbifolds. We briefly discuss the CFT duals. We end the discussion with conical spaces created by moving point particles and describe the scenario in which two such particles with sufficient energy to form a BTZ black hole. The dual CFT is left as an open problem.

In Section 12 we conclude with a discussion of some open problems. 


\section{Construction of classical solutions}

The aim of this section will be to construct the classical solution representing the five-dimensional black hole in [44]. Rather than presenting the solution and showing that it solves the low energy equations of type II superstring, we will describe some aspects of the art of solution-building. There are many excellent reviews of this area (see, for example, [15, 46, 47, 48, 49, 50, 51, 52, 53], other general reviews on black holes in string/M theories include [54, 55, 56]), so we shall be brief. The method of construction of various classical solutions, we will see, will throw light on the microscopic configurations corresponding to these solutions.

Two widely used methods for construction of classical solutions are

(a) the method of harmonic superposition

(b) $O(d, d)$ transformations

We will mainly concentrate on the first one below. For a more detailed account including the second method, see, e.g., [50].

As is well-known by now, classical solutions of type II string theories can be obtained from those of M-theory [57, 58] through suitable compactification and dualities. We will accordingly start with classical solutions of M-theory, or alternatively, of 11-dimensional supergravity.

We should note two important points:

(a)For classical supergravity description of these solutions to be valid, we need the curvature to be small (in the scale of the 11-dimensional Planck length $l_{11}$ for solutions of M-theory, or of the string length $l_{s}$ for string theories)

(b) Since various superstring theories are defined (through perturbation theory) only in the (respective) weakly coupled regimes, in order to meaningfully talk about classical solutions of various string theories we need the string coupling also to be small.

For the RR charged type II solutions (charge Q) that we will describe below, both the above conditions can be met if $Q \gg 1 / g_{s} \gg 1$ (that is, $g_{s} Q \gg 1, g_{s} \ll 1$ ).

\subsection{Classical solutions of M-theory}

The massless modes of M-theory are those of 11-dimensional supergravity: the metric $G_{M N}$, the gravitino $\psi_{M}$ and a three-form $A_{M N P}^{(3)}, M=0,1, \ldots, 10$.

The (bosonic part of the) classical action is

$$
S_{11}=\frac{1}{2 \kappa_{11}^{2}} \int d^{11} x\left[\sqrt{-G}\left(R-\frac{1}{48}\left(d A^{(3)}\right)^{2}\right)-\frac{1}{6} A^{(3)} \wedge d A^{(3)} \wedge d A^{(3)}\right]
$$

There are two basic classical solutions of this Lagrangian, the M2 and M5 branes, whose intersections account for most stable supersymmetric solutions of M-theory [59, 60, 477. 


\subsubsection{The 2-brane solution of M-theory: M2}

It will suffice for us to describe only the M2-brane solution [61] in some detail. Statement of the problem: we want to find (a) a relativistic 2-brane solution of (2.1) (say stretching along $x^{1,2}$ ) with (b) some number of unbroken supersymmetries.

Condition (a) implies that the solution must have a $S O(2,1)_{0,1,2} \times S O(8)_{3,4, \ldots, 10}$ symmetry, together with translational symmetries along $x^{0,1,22}$. This uniquely leads to

$$
\begin{aligned}
& d s_{11}^{2}=e^{2 A_{1}(r)} d x^{\mu} d x_{\mu}+e^{2 A_{2}(r)} d x^{m} d x_{m} \\
& A^{(3)}=e^{A_{3}(r)} d t \wedge d x^{1} \wedge d x^{2}
\end{aligned}
$$

where $\mu=0,1,2$ denote directions parallel to the world-volume and $m=3, \ldots, 9,10$ denote the transverse directions. $r^{2} \equiv x^{m} x_{m}$.

Condition (b) implies that there should exist a non-empty set of supersymmetry transformations $\epsilon$ preserving the solution (2.2); in particular the gravitino variation

$$
\begin{aligned}
\delta_{\epsilon} \psi_{M} & =D_{M} \epsilon+\frac{1}{288}\left(\Gamma_{M}^{N P Q R}-8 \delta_{M}^{N} \Gamma^{P Q R}\right) F_{N P Q R} \epsilon=0, \text { where } \\
D_{M} \epsilon & \equiv\left(\partial_{M}+\frac{1}{4} \omega_{M}^{B C} \Gamma_{B C}\right) \epsilon
\end{aligned}
$$

must vanish for some $\epsilon$ 's.

It is straightforward to see that Eqn. (2.3) vanishes for $M=\mu$ (world volume directions) if

$$
\begin{aligned}
\partial_{\mu} \epsilon & =0, \\
A_{3} & =3 A_{1} \\
\text { and } \Gamma^{\hat{0} \hat{1} \hat{2}} \epsilon & =\epsilon
\end{aligned}
$$

where the caret ^ denotes local Lorenz indices. (Flipping the sign of $A^{(3)}$ would correspond to $-\epsilon$ on the right hand side of the last line of (2.4): this would correspond to an anti-brane solution in our convention.)

The $M=m$ (transverse) components of (2.3) give rise to the further conditions

$$
\begin{aligned}
A_{1} & =-2 A_{2} \\
\epsilon & =e^{A_{3} / 6} \epsilon_{0}
\end{aligned}
$$

\section{Harmonic equation}

\footnotetext{
${ }^{2}$ The subscripts denote which directions are acted on by the $S O$ groups. We denote spacetime coordinates by $x^{M}, M=0,1, \ldots, 10$.
} 
The equations (2.4) and (2.5) fix the three functions $A_{i}$ in (2.2) in terms of just one function, say $A_{3}$. It is easy to determine it by looking at the equation of motion of the three-form potential:

$$
\partial_{M}\left(\sqrt{-g} F^{M N P Q}\right)+\frac{1}{2 .(4 !)^{2}} \epsilon^{N P Q A B C D E F G H} F_{A B C D} F_{E F G H}=0
$$

The second term is clearly zero for our ansatz (2.2) for $A^{(3)}$. The first term, evaluated for $(P, Q, R)=(0,1,2)$ gives to

$$
\partial_{m} \partial_{m}\left(e^{-A_{3}}\right)=0
$$

Thus, the full M2 solution is given by

$$
\begin{aligned}
d s_{11}^{2} & =H^{1 / 3}\left[H^{-1} d x^{\mu} d x_{\mu}+d x^{m} d x_{m}\right] \\
A^{(3)} & =H^{-1} d t \wedge d x^{1} \wedge d x^{2}
\end{aligned}
$$

where $H=H(r)$ satisfies the harmonic equation in the transverse coordinates

$$
\partial_{m} \partial_{m} H=0
$$

The simplest solution for $H$, in an asymptotically flat space, is given by

$$
H=1+k / r^{6}
$$

Clearly, multi-centred solutions are also allowed:

$$
H=1+\sum_{i} \frac{k_{i}}{\left|\vec{x}-\overrightarrow{x_{i}}\right|^{6}}
$$

where $\vec{x}$ denotes the transverse directions $x^{m}$.

We note that, the constant, 1 , in (2.10) is essentially an integration constant. Clearly, it can also be zero; such choices have led to M/string theory solutions involving AdS spaces. The point of this remark is to emphasize that the nearhorizon geometry $(r \rightarrow 0)$, important in the context of AdS/CFT correspondence [42, 62, 63], in which $H=k / r^{6}$, corresponds to a complete solution in its own right without the appendage of the asymptotically flat regions. We will return to the AdS/CFT correspondence many times in this as well as later Sections.

\section{$\underline{A D M}$ mass}

The integration constant $k$ in $(2.10)$ affects the asymptotic fall-off of the metric as well as of the field strength, and is related to the ADM mass (per unit area of the 2-brane) $M$ and to the gauge charge (per unit area) $q$. Using the definitions ${ }^{3}$

$$
\begin{aligned}
M & =\int_{S^{7}} d^{7} \Sigma^{m}\left(\partial^{n} h_{m n}-\partial_{m} h\right), \\
q & =\int_{S^{7}} d^{7} \Sigma^{m} F_{m 012}, \\
\text { we get } M & =6 k \Omega_{7}=q
\end{aligned}
$$

\footnotetext{
${ }^{3}$ We follow the normalizations in [45] which differ from, e.g. [48].
} 
Here $S^{7}$ represents the sphere at $r^{2}=x^{m} x_{m}=\infty,{ }^{4} h_{M N} \equiv g_{M N}-\eta_{M N}, h \equiv$ $\sum_{M=1}^{10} h_{M M}$, and $\Omega_{n} \equiv 2 \pi^{(n+1) / 2} / \Gamma\left(\frac{n+1}{2}\right)$ is the volume of the unit sphere $S^{n}$.

\section{$\underline{B P S \text { nature }}$}

The mass-charge equality in the last equation (2.14) is characteristic of a "BPS solution". We provide a very brief introduction below. The 11-dimensional supersymmetry algebra [64] is

$$
\{Q, Q\}=C\left(\Gamma^{M} P_{M}+\Gamma^{M N} U_{M N}+\Gamma^{M N P Q R} V_{M N P Q R}\right),
$$

where $C$ is the charge conjugation matrix and $P, U$ and $V$ are various central terms. When (2.15) is evaluated [65] for the above M2 solution, we get

$$
\frac{1}{V_{2}}\left\{Q_{\alpha}, Q_{\beta}\right\}=\left(C \Gamma^{\hat{0}}\right)_{\alpha \beta} M+\left(C \Gamma^{\hat{1} \hat{2}}\right)_{\alpha \beta} Q,
$$

where we have used the notation

$$
P_{\hat{0}}=V_{2} M, \quad U_{\hat{1} \hat{2}}=V_{2} q,
$$

$V_{2}$ being the spatial volume of the 2-brane (assumed compactified on a large $T^{2}$ ).

Now, the positivity of the $Q^{2}$ operator implies that $M \geq q$ where the inequality is saturated when the right hand side of (2.16) has a zero eigenvector. For our solution (2.8), we see from (2.4) that the unbroken supersymmetry transformation parameter satisfies

$$
\left(1-\Gamma^{\hat{0} \hat{1} \hat{2}}\right) \epsilon=0
$$

This clearly leads to $M=q$. This is a typical example of how classical solutions with (partially) unbroken supersymmetries satisfy the extremality condition mass= charge.

We note here that the remaining half of the supersymmetry transformations, the complement of the ones in (2.18), are non-linearly realized in the M2 geometry and can be regarded as spontaneously broken supersymmetries. Interestingly, the supersymmetry variations under these transformations vanish in the near-horizon limit which has the geometry [66] $A d S_{4} \times S^{7}$. As a result the broken supersymmetry transformations reemerge as unbroken, leading to an enhancement of the number of supersymmetry charges $16 \rightarrow 32$ in the near-horizon limit, a fact that plays a crucial role in the AdS/CFT correspondence.

Identification as a "Black brane"

The M2-brane itself has a black hole geometry. If we compactify the directions 1,2 on a 2 -torus, we have a black hole solution in the remaining nine extended dimensions. The compactified solution is constructed by placing the multiple centres

\footnotetext{
${ }^{4}$ The total ADM mass, which diverges, includes integrals over $x^{1,2}$ as well; we ignore them here since we are interested in the mass per unit area. Similar remarks apply to the charge.
} 
$\overrightarrow{x_{i}}$ in $(2.11)$ at the sites of a lattice defining the 2-torus. The horizon is situated at $r=0$. The detailed geometry has been discussed in many places, e.g in [45]. Since our main object of interest is the five-dimensional black hole, and we will use the M2-brane as essentially a building block for that solution, we defer the geometrical discussion till we discuss the latter.

Without compactification too, the above solution is "black", but it has extensions in 1,2 directions and is called a black 2-brane.

\subsubsection{Intersecting M2-branes}

We will now use the above solution as a building block to construct more complicated solutions corresponding to intersecting branes.

$\underline{M 2 \perp M 2}$

We consider first two orthogonal M2 branes, along $x^{1,2}$ and $x^{3,4}$ respectively. The geometry of the solution corresponds to a spacetime symmetry consisting of rotations $S O(2)_{1,2} \times S O(2)_{3,4} \times S O(6)_{5,6,7,8,9,10}$ plus Killing vectors $\left(\partial_{t}, \partial_{1}, \ldots, \partial_{4}\right)$. This leads to

$$
\begin{aligned}
& d s_{11}^{2}=e^{2 A_{1}}\left(-d t^{2}\right)+e^{2 A_{2}}\left(d x_{1}^{2}+d x_{2}^{2}\right)+e^{2 A_{3}}\left(d x_{3}^{2}+d x_{4}^{2}\right)+e^{2 A_{4}} d x_{i} d x_{i} \\
& A^{(3)}=e^{A_{5}} d t \wedge d x^{1} \wedge d x^{2}+e^{A_{6}} d t \wedge d x^{3} \wedge d x^{4}
\end{aligned}
$$

Now, as before, the desire to have a BPS solution leads to existence of unbroken supersymmetry, or $\delta_{\epsilon} \psi_{M}=0$. This now yields four different type of equations, depending on whether the index $M$ is $0,\{1,2\},\{3,4\}$ or the rest. These express the six functions above in terms of two independent functions $H_{1}, H_{2}$. These functions turn out to harmonic in the common transverse directions when one imposes closure of SUSY algebra or equation of motion. The solution ultimately is

$$
\begin{aligned}
& d s_{11}^{2}=\left(H_{1} H_{2}\right)^{1 / 3}\left[-\frac{d t^{2}}{H_{1} H_{2}}+\frac{d x_{1}^{2}+d x_{2}^{2}}{H_{1}}+\frac{d x_{3}^{2}+d x_{4}^{2}}{H_{2}}+d x_{i} d x_{i}\right] \\
& A^{(3)}=\frac{1}{H_{1}} d t \wedge d x^{1} \wedge d x^{2}+\frac{1}{H_{2}} d t \wedge d x^{3} \wedge d x^{4}
\end{aligned}
$$

The above is an example of "harmonic superposition of branes". (see, e.g., 60]).

\section{Delocalized nature of the solution}

We note that the ansatz above (2.19), as well as the solution (2.20), represent a "delocalized solution". A localized $M 2 \perp M 2$ intersection would destroy translational symmetries along the spatial world-sheet of both the 2-branes. The subject of localized intersection is interesting in its own right (see, e.g. 67] which is especially relevant to the D1-D5 system), although we do not have space to discuss them here. The delocalization here involves "smearing" the first M2 solution along the directions $x^{3}, x^{4}$ (by using a continuous superposition in (2.11), see e.g. 477), and "smearing" the second M2 solution along $x^{1}, x^{2}$. 
$\underline{M 2 \perp M 2 \perp M 2}$

Extending the above method, we get the following supergravity solution for three orthogonal M2-branes, extending respectively along $x^{1,2}, x^{3,4}$ and $x^{5,6}$ :

$$
\begin{aligned}
d s_{11}^{2}= & \left(H_{1} H_{2} H_{3}\right)^{1 / 3}\left[\left(H_{1} H_{2} H_{3}\right)^{-1}\left(-d t^{2}\right)+H_{1}^{-1}\left(d x_{1}^{2}+d x_{2}^{2}\right)\right. \\
+ & \left.H_{2}^{-1}\left(d x_{3}^{2}+d x_{4}^{2}\right)+H_{3}^{-1}\left(d x_{5}^{2}+d x_{6}^{2}\right)+d x_{i} d x_{i}\right] \\
A^{(3)}= & H_{1}^{-1} d t \wedge d x^{1} \wedge d x^{2}+H_{2}^{-1} d t \wedge d x^{3} \wedge d x^{4} \\
& +H_{3}^{-1} d t \wedge d x^{5} \wedge d x^{6}
\end{aligned}
$$

\subsection{The $6 \mathrm{D}$ black string solution of IIB on $T^{4}$}

In the following we will construct solutions of type II string theories using the above M-theory solutions by using various duality relations which we will describe as we go along. For an early account of black p-brane solutions in string theory, see [68].

We apply the transformation $T_{567} R_{10}$ to the $M 2 \perp M 2$ solution (2.20):

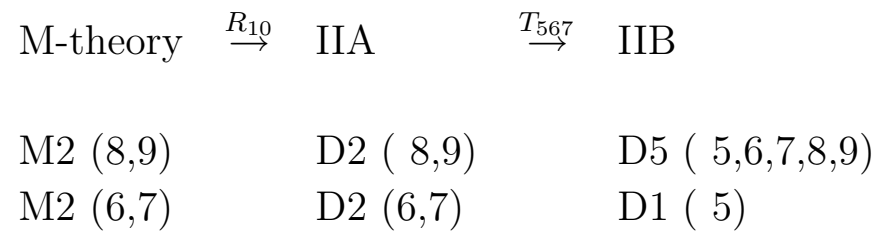

The first transformation $R_{10}$ denotes the reduction from M-theory to type IIA. To do this, one first needs to compactify the $M 2 \perp M 2$ solution along $x^{10}$ (by using the multi-centred harmonic functions, with centres separated by a distance $2 \pi R_{10}$ along $\left.x^{10}\right)$. Essentially, at transverse distances large compared to $R_{10}$, this amounts to replacement of the harmonic function $1 / r^{4}$ by $1 / r^{3}$ and a suitable modification of the integration constant to reflect the appropriate quantization conditions. At this stage, one still has 11-dimensional fields. To get to IIA fields, we use the reduction formula

$$
\begin{aligned}
d s_{11}^{2} & =\exp [-2 \phi / 3] d s_{10}^{2}+\exp [4 \phi / 3]\left(d x^{10}+C_{\mu}^{(1)} d x^{\mu}\right)^{2} \\
A & =B \wedge d x^{10}+C^{(3)}
\end{aligned}
$$

It is instructive to verify at this stage that the classical D2 solutions do come out of the M2-brane after these transformations. We use the notation $C^{(n)}$ for the $n$-Form Ramond-Ramond (RR) potentials in type II theories.

The second transformation $T_{567}$ involves a sequence of $\mathrm{T}$-dualities (for a recent account of T-duality transformations involving RR fields, see [69]). We denote by $T_{m}$ T-duality along the direction $x^{m} . T_{567}$ denotes $T_{5} T_{6} T_{7}$.

The final transformation, not explicitly written in the above table, is to wrap $x^{6,7,8,9}$ on $T^{4}$. We will denote the volume of the $T^{4}$ by

$$
V_{T^{4}} \equiv \alpha^{2}(2 \pi)^{4} \tilde{v}
$$


Assuming the number of the two orthogonal sets of M2-branes to be $Q_{5}, Q_{1}$ respectively, the final result is: $Q_{5}$ strings from wrapping D5 on $T^{4}$ and $Q_{1}$ D-strings. This is the D1-D5 system in IIB supergravity ${ }^{5}$, characterized by the following solution:

$$
\begin{aligned}
d s_{10}^{2} & =f_{1}^{-1 / 2} f_{5}^{-1 / 2}\left(-d t^{2}+d x_{5}^{2}\right)+f_{1}^{1 / 2} f_{5}^{1 / 2} d x_{i} d x^{i}+f_{1}^{1 / 2} f_{5}^{-1 / 2} d x_{a} d x^{a} \\
C_{05}^{(2)} & =-\frac{1}{2}\left(f_{1}^{-1}-1\right) \\
F_{i j k}^{(3)} & =\epsilon_{i j k l} \partial_{l} f_{5}, F^{(3)}=d C^{(2)} \\
e^{-2 \phi} & =f_{5} f_{1}^{-1} \\
f_{1,5} & =\left(1+r_{1,5}^{2} / r^{2}\right)
\end{aligned}
$$

Here $C^{(2)}$ is the 2-form RR gauge potential of type IIB string theory. The parameters $r_{1}, r_{5}$ are defined in terms of $Q_{1}, Q_{5}$, see Eqns. (2.28).

\section{Spacetime Symmetry}

The spacetime symmetry $\mathcal{S}$ of the above solution is:

$$
\mathcal{S}=S O(1,1) \times S O(4)_{E} \times ' S O(4)_{I} ’
$$

where $S O(1,1)$ refers to directions $0,5, S O(4)_{E}$ to directions $1,2,3,4$ ( $E$ for external) ' $S O(4)_{I}$ ' to directions $6,7,8,9$ ( $I$ stands for internal; the quotes signify that the symmetry is broken by wrapping the directions on a four-torus although for low energies compared to the inverse radii it remains a symmetry of the supergravity solution).

\section{Supersymmetry}

The unbroken supersymmetry can be read off either by recalling those of the Mtheory solution and following the dualities or by solving the Killing spinor equations (analogous to (2.3)). The result is:

$$
\begin{aligned}
\Gamma^{056789} \epsilon_{L} & =\epsilon_{R} \\
\Gamma^{05} \epsilon_{L} & =\epsilon_{R}
\end{aligned}
$$

The first line corresponds to the unbroken supersymmetry appropriate for the D5brane (extending in 5,6,7,8,9 directions). The second line refers to the D1-brane. (The superscripts in $\Gamma^{a b . .}$ denote local Lorenz indices like in (2.3), although we have dropped the carets.)

To solve Eqn. (2.26), we recast (2.26) as

$$
\Gamma^{6789} \epsilon_{L}=\epsilon_{L}, \epsilon_{R}=\Gamma^{05} \epsilon_{L}
$$

\footnotetext{
${ }^{5}$ Strictly speaking, we should wrap the D1-D5 string on a large circle to avoid the GregoryLaflamme instability [70].
} 
Since $\epsilon_{L}$ has 16 independent real components to begin with, the first equation cuts it down by a half, thus leaving 8 real components. These, by virtue of the second equation, completely determine $\epsilon_{R}$, thus leaving no further degrees of freedom.

Thus, there are eight unbroken real supersymmetries. Recall that type II string theory has 32 supersymmetries (i.e., $\epsilon_{L}, \epsilon_{R}$ each has 16 independent real components). In the near-horizon limit, eight of the broken supersymmetries reemerge as unbroken.

\subsection{The extremal 5D black hole solution}

Let us now compactify $x^{5}$ along a circle of radius $R_{5}$ and wrap the above solution along $x^{5}$ to get a spherically symmetric object in five dimensions. Let us also "add" gravitational waves (denoted $W$ ) moving to the "left" along $x^{5}$. This gives us the BPS version [71, 44] of the five-dimensional black hole. Adding such a wave can be achieved either (a) by applying the Garfinkle-Vachaspati transformation [72 to the black string solution (2.24) and wrapping it on $S^{1}$, or (b) augmenting the $M 2 \perp M 2$ solution $(2.20)$ by a third, transverse, set of M2-branes along $x^{5,10}$ (cf. (2.21)), and passing it through the same sequence of transformations $T_{567} R_{10}$ as before (see the Table in Section 2.2), with the result that the third set of M2 branes becomes a gravitational wave (= momentum mode) along the $x^{5}$-circle:

\begin{tabular}{|c|c|c|c|c|}
\hline M-theory & $\stackrel{R_{10}}{\longrightarrow}$ & IIA & $\stackrel{T_{567}}{\longrightarrow}$ & IIB \\
\hline M2 $(8,9)$ & & D2 $(8,9)$ & & D5 $(5,6,7,8,9)$ \\
\hline M2 $(6,7)$ & & D2 $(6,7)$ & & D1 ( 5$)$ \\
\hline M2 $(5,10)$ & & $\operatorname{NS1}(5)$ & & $\mathrm{W}(5)$ \\
\hline
\end{tabular}

The last transformation essentially reflects the fact that T-duality changes winding modes to momentum modes. ( $W$ denotes a gravitational wave and not a winding mode.)

The final configuration corresponds to D5 branes along $x^{5,6,7,8,9}$ and D1 branes along $x^{5}$, with a non-zero amount of (left-moving) momentum. If the number of the three sets of M2 branes are $Q_{1}, Q_{5}$ and $N$ respectively, then these will correspond to the numbers of D1-, D5-branes and the quantized left-moving momentum respectively.

The solution for the extremal five-dimensional D1-D5 black hole is thus given by

$$
\begin{aligned}
d s_{10}^{2}= & f_{1}^{-1 / 2} f_{5}^{-1 / 2}\left(-d u d v+\left(f_{n}-1\right) d u^{2}\right) \\
& +f_{1}^{1 / 2} f_{5}^{1 / 2} d x_{i} d x^{i}+f_{1}^{1 / 2} f_{5}^{-1 / 2} d x_{a} d x^{a} \\
C_{05}^{(2)}= & -\frac{1}{2}\left(f_{1}^{-1}-1\right) \\
F_{i j k}^{(3)}= & \epsilon_{i j k l} \partial_{l} f_{5}
\end{aligned}
$$




$$
\begin{aligned}
e^{-2 \phi} & =f_{5} f_{1}^{-1} \\
f_{1,5, n} & =\left(1+\left(\frac{r_{1,5, n}}{r}\right)^{2}\right)
\end{aligned}
$$

The parameters $r_{1}, r_{5}, r_{n}$ are defined in terms of $Q_{1}, Q_{5}, N$ respectively, see Eqn. (2.28).

\section{$\underline{\text { Symmetries }}$}

Curling up $x^{5}$ and adding momentum along it reduces the spacetime symmetry and supersymmetry of the solution (2.27), compared to (2.25),(2.26). Thus the spacetime symmetry is $S O(4)_{E} \times$ ' $\mathrm{SO}(4)_{I}$ ' while the number of supersymmetries is reduced to four due to an additional condition on the Killing spinor: $\Gamma^{05} \epsilon_{L, R}=\epsilon_{L, R}$.

\section{Charge quantization}

The parameters $r_{1,5, n}^{2}$ in (2.27) are related to the integer-quantized charges $Q_{1,5}$ and momentum $N$ by

$$
\begin{aligned}
& r_{1}^{2}=c_{1} Q_{1}, c_{1}=\frac{4 G_{N}^{5} R_{5}}{\pi \alpha^{\prime} g_{s}}=\frac{g_{s} \alpha^{\prime}}{\tilde{v}} \\
& r_{5}^{2}=c_{5} Q_{5}, c_{5}=g_{s} \alpha^{\prime} \\
& r_{n}^{2}=c_{n} N, c_{n}=\frac{4 G_{N}^{5}}{\pi R_{5}}=\frac{g_{s}^{2} \alpha^{\prime 2}}{\tilde{v} R_{5}^{2}}
\end{aligned}
$$

where

$$
G_{N}^{5}=\frac{G_{N}^{10}}{\left(2 \pi R_{5} V_{T^{4}}\right)}=\frac{\pi g_{s}^{2} \alpha^{\prime 2}}{4 \tilde{v} R_{5}^{2}}
$$

In the above we have used (1.1), (2.23). For a detailed discussion of quantization

conditions like (2.28), see, e.g. [73, 48, 45]. Here $G_{N}^{d}$ denotes the $d$-dimensional Newton's constant. $V_{T^{4}}$ is the volume of the four-torus in the directions $x^{6,7,8,9}$, while $R_{5}$ is the radius of the circle along $x^{5}$.

\section{Explicit five-dimensional form}

The background (2.27) is written in a ten-dimensional form. It is easy to derive the five-dimensional metric and other fields. For the five-dimensional Einstein metric we use the reduction formula

$$
d s_{10}^{2}=e^{2 \chi} d x_{a} d x^{a}+e^{2 \psi}\left(d x_{5}+A_{\mu} d x^{\mu}\right)^{2}+e^{-(8 \chi+2 \psi+\phi) / 3} d s_{5}^{2}
$$

(the first two exponential factors are simply the definitions of the scalars $\chi, \psi$; the factor in front of $d s_{5}^{2}$ can be found easily by demanding that $d s_{5}^{2}$ is the fivedimensional Einstein metric). Here $\mu=1,2,3,4$.

Using (2.30), the five-dimensional Einstein metric is given by

$$
\begin{aligned}
d s_{5}^{2} & =-f^{-2 / 3}(r) d t^{2}+f^{1 / 3}(r)\left(d r^{2}+r^{2} d \Omega_{3}^{2}\right) \\
f(r) & =f_{1}(r) f_{5}(r) f_{n}(r)
\end{aligned}
$$


where $f_{1,5, n}(r)$ are defined in (2.27).

\section{Area and Entropy}

The above metric has a horizon at $r=0$, which has a finite radius $R_{h}$ and a finite area $A_{h}$, given by

$$
A_{h}=2 \pi^{2} R_{h}^{3}, R_{h}=\left(r_{1} r_{5} r_{n}\right)^{1 / 3}
$$

The Bekenstein-Hawking entropy (1.10), is given by

$$
S=2 \pi^{2} \frac{\sqrt{c_{1} c_{5} c_{n}}}{4 G_{N}^{5}} \sqrt{Q_{1} Q_{5} N}=2 \pi \sqrt{Q_{1} Q_{5} N}
$$

where in the second step we have used

$$
\sqrt{c_{1} c_{5} c_{n}}=\frac{4 G_{N}^{5}}{\pi}
$$

which follows easily from (2.28). The fact that all "moduli" like the coupling and radii disappear from (2.33), and that the entropy is ultimately given only in terms of quantized charges, is remarkable.

We defer a discussion of the geometry of this solution till the next section 2.4 where we describe the non-extremal version.

\subsection{Non-extremal five-dimensional black hole}

We have explained above how to construct from first principles the BPS (hence extremal) version of the 5D black hole solution. We will now present an algorithm (without proof and specialized to intersections of M2) of how to generalize these constructions to their non-extremal (nonsupersymmetric) versions [74]. A heuristic motivation for this algorithm is presented in appendix B.

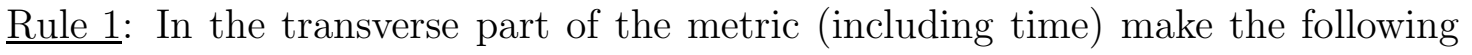
substitution:

$$
\begin{aligned}
d t^{2} & \rightarrow h(r) d t^{2}, \quad d x^{i} d x_{i} \rightarrow h^{-1}(r) d r^{2}+r^{2} d \Omega_{d-1}^{2} \\
h(r) & =1-\mu / r^{d-2}
\end{aligned}
$$

with the harmonic function now defined as

$$
H(r)=1+\tilde{Q} / r^{d-2}
$$

where $\tilde{Q}$ is a combination of the non-extremality parameter $\mu$ and some "boost" angle $\delta$ :

$$
\tilde{Q}=\mu \sinh ^{2} \delta
$$

(for multicentred solutions, $\tilde{Q}_{i}=\mu \sinh ^{2} \delta_{i}$.) 
Rule 2:

In the expression for $F_{4}=d A$, make the substitution

$$
\begin{aligned}
H & \rightarrow \tilde{H}(r)=1+\frac{\bar{Q}}{r^{d-2}+\tilde{Q}-\bar{Q}}=\left(1-\frac{\bar{Q}}{r^{d-2}} H^{-1}\right)^{-1}, \\
\bar{Q} & =\mu \sinh \delta \cosh \delta
\end{aligned}
$$

Applying this rule to the $M 2 \perp M 2 \perp M 2$ case (2.21), we get

$$
\begin{aligned}
d s_{11}^{2} & =\left(H_{1} H_{2} H_{3}\right)^{-1 / 3}\left[-H_{1} H_{2} H_{3} h d t^{2}+H_{1}\left(d y_{1}^{2}+d y_{2}^{2}\right)\right. \\
& +H_{2}\left(d y_{3}^{2}+d y_{4}^{2}\right)+H_{3}\left(d y_{5}^{2}+d y_{6}^{2}\right)+h^{-1} d r^{2} \\
& \left.+r^{2} d \Omega_{d-1}^{2}\right]
\end{aligned}
$$

The rest of the story is similar to the BPS case described in the previous subsection. Namely, we apply the duality transformation $T_{567} R_{10}$ as in the Table in Section 2.3: by first reducing the M-theory solution (2.38) to IIA and then T-dualizing to IIB, and finally wrapping it on $T^{4} \times S^{1}$.

Under the reduction from M-theory to type IIA in ten dimensions, we get

$$
\begin{aligned}
e^{-2 \phi} & =f_{1} f_{5}^{-1} \\
d s_{10}^{2} & =f_{1}^{-1 / 2} f_{5}^{-1 / 2}\left[-d t^{2}+d x_{5}^{2}\right. \\
& \left.+(1-h)\left(\cosh \alpha_{n} d t+\sinh \alpha_{n} d x_{5}\right)^{2}\right] \\
& +f_{1}^{1 / 2} f_{5}^{1 / 2}\left(\frac{d r^{2}}{h}+r^{2} d \Omega_{3}^{2}\right)+F_{1}^{1 / 2} F_{5}^{-1 / 2} d x_{a} d x^{a} \\
h & =1-r_{0}^{2} / r^{2}
\end{aligned}
$$

where $a=6, . ., 9,\left(r, \Omega_{3}\right)$ are polar coordinates for $x^{1,2,3,4} \cdot f_{1}, f_{5}\left(\right.$ also $f_{n}$ in (2.40) $)$ are defined as in (2.27), except that the parameters $r_{1}, r_{5}$ (also $r_{n}$ ) are no more defined by (2.28), but by their non-extremal counterparts (2.40), (2.41). The parameter $r_{0}^{2}$ is the same as the non-extremality parameter $\mu$ of (2.35), while $\alpha_{1,5, n}$ are related to the boost angle of (2.36).

This is still a IIA solution. In order to get the IIB version, we have to apply the sequence $T_{567}$. We omit the details here which are fairly straightforward. At the end, after we further use the Kaluza-Klein reduction (2.30) we get the following five-dimensional Einstein metric [75]:

$$
\begin{aligned}
d s_{5}^{2} & =-h f^{-2 / 3} d t^{2}+f^{1 / 3}\left(\frac{d r^{2}}{h}+r^{2} d \Omega_{3}^{2}\right) \\
f & =f_{1} f_{5} f_{n}=\left(1+r_{1}^{2} / r^{2}\right)\left(1+r_{5}^{2} / r^{2}\right)\left(1+r_{n}^{2} / r^{2}\right) \\
r_{1,5, n}^{2} & =r_{0}^{2} \sinh ^{2} \alpha_{1,5, n}
\end{aligned}
$$

There are six independent parameters of the metric: $\alpha_{1,5, n}, r_{0}, R_{5}, \tilde{v} \equiv V_{T^{4}} /\left(2 \pi l_{s}\right)^{4}$ $\left(l_{s}=\sqrt{\alpha^{\prime}}\right)$. The boost angles and the non-extremality parameters are related to 
the three charges and the mass $M$ as follows: $\left(F^{(3)} \equiv d C^{(2)}\right)$

$$
\begin{aligned}
Q_{1} & =\frac{V}{4 \pi^{2} g} \int e^{\phi} * F^{(3)}=\frac{r_{0}^{2} \sinh 2 \alpha_{1}}{2 c_{1}} \\
Q_{5} & =\frac{1}{4 \pi^{2} g_{s}} \int F^{(3)}=\frac{r_{0}^{2} \sinh 2 \alpha_{5}}{2 c_{5}} \\
N & =\frac{r_{0}^{2} \sinh 2 \alpha_{n}}{2 c_{n}} \\
M & =\frac{R_{5} \tilde{v} r_{0}^{2}}{2\left(\alpha^{\prime}\right)^{2} g_{s}^{2}}\left(\cosh 2 \alpha_{1}+\cosh 2 \alpha_{5}+\cosh 2 \alpha_{n}\right)
\end{aligned}
$$

There is another very interesting representation of the above-mentioned six param-

eters in terms of what looks like brane-, antibrane-numbers and left-, right-moving momenta:

$$
N_{1, \overline{1}}=\frac{r_{0}^{2} e^{ \pm 2 \alpha_{1}}}{4 c_{1}}, N_{5, \overline{5}}=\frac{r_{0}^{2} e^{ \pm 2 \alpha_{5}}}{4 c_{5}}, N_{L, R}=\frac{r_{0}^{2} e^{ \pm 2 \alpha_{n}}}{4 c_{n}}
$$

The coefficients $c_{1}, c_{5}, c_{n}$ are as in (2.28). Clearly

$$
N_{1}-N_{\overline{1}}=Q_{1}, N_{5}-N_{\overline{5}}=Q_{5}, N_{L}-N_{R}=N .
$$

The extremal limit corresponds to taking $r_{0} \rightarrow 0, \alpha_{1,5, n} \rightarrow \infty$ keeping the charges $Q_{1,5}, N$ finite. We comment on the brane-antibrane interpretation in Section 2.4.2.

\subsubsection{Geometry}

It is easy to see that the above solution is a five-dimensional black hole, with horizon at $r=r_{0}$. The horizon has a finite area $A_{h}$, given by

$$
\begin{aligned}
A_{h} & =2 \pi^{2} r_{0}^{3} \cosh \alpha_{1} \cosh \alpha_{5} \cosh \alpha_{n} \\
& =8 \pi G_{N}^{5}\left(\sqrt{N}_{1}+\sqrt{N}_{\overline{1}}\right)\left(\sqrt{N}_{5}+\sqrt{N}_{\overline{5}}\right)\left(\sqrt{N}_{L}+\sqrt{N}_{R}\right)
\end{aligned}
$$

Here we have used (2.34) and (2.42).

The fact that the horizon has a finite area indicates that the singularity lies "inside" $r=r_{0}$. It is not at $r=0$, however, which corresponds to the inner horizon (where light-cones "flip" the second time as one travels in). To locate the singularity one needs to use other coordinate patches which extend the manifold further "inside". The singularity is time-like and the Carter-Penrose diagram (Fig 1) is similar to that of the non-extremal Reissner-Nordstrom metric (see (1.3),(1.4)). The inner and outer horizons (cf. (1.4)) in the present case are $r_{-}=0, r_{+}=r_{0}$.

\subsubsection{Hawking temperature and Bekenstein-Hawking entropy}

By using the formula (A.9) we get the following Hawking temperature $(\hbar=1)$ (see Appendix A for details)

$$
\frac{1}{T_{H}}=2 \pi r_{0} \cosh \left(\alpha_{1}\right) \cosh \left(\alpha_{5}\right) \cosh \left(\alpha_{n}\right)
$$




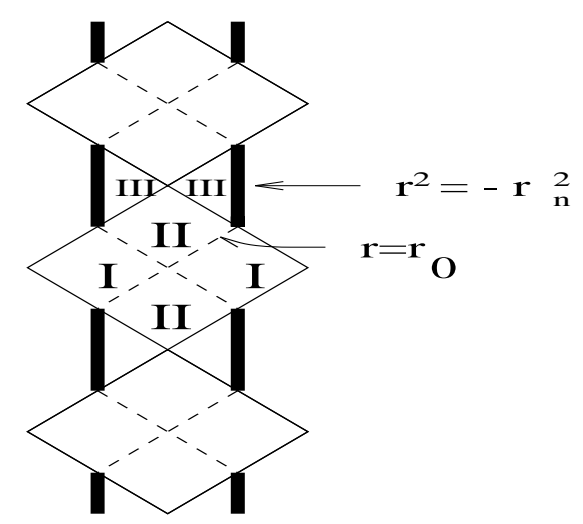

Figure 1: Carter-Penrose diagram for the non-extremal 5D black hole

We will compare this with the CFT result for the temperature (4.31) in Section 1 (see also Section 8).

By using the formula $S=A_{h} / 4 G_{N}^{5}$ (cf. (1.10)) and (2.44), we get

$$
S_{\mathrm{BH}}=2 \pi\left(\sqrt{N}_{1}+\sqrt{N}_{\overline{1}}\right)\left(\sqrt{N}_{5}+\sqrt{N}_{\overline{5}}\right)\left(\sqrt{N}_{L}+\sqrt{N}_{R}\right)
$$

Of course, the extremal entropy (2.33) corresponds to the special case $N_{R}=N_{\overline{1}}=$ $N_{\overline{5}}=0$ (use (2.43)). A somewhat more general case is when $N_{\overline{1}}={\sqrt{N_{\overline{5}}}}=0, N_{R} \neq$ 0 ; the entropy in that case is given by

$$
S_{\mathrm{BH}}=2 \pi \sqrt{Q_{1} Q_{5}}\left(\sqrt{N_{L}}+\sqrt{N_{R}}\right)
$$

The entropy formulae (2.33) and (2.46) are U-duality invariant, in the following sense. Consider an $S(3)$ subgroup of the U-duality group of type IIB on $T^{5}$, which permutes the three charges $Q_{1}, Q_{5}$ and $N$. Such an $S(3)$ is generated by

(a) $T_{6789}$ which sends $Q_{1} \rightarrow Q_{5}, Q_{5} \rightarrow Q_{1}, N \rightarrow N$, and

(b) $T_{9876} S T_{65}$ which sends $Q_{1} \rightarrow Q_{5}, Q_{5} \rightarrow N, N \rightarrow Q_{1}$.

The entropy formula (2.33) remains invariant under these permutations. Since the "anti"-objects are also permuted among each other by these U-duality transformations, we can say that the entropy formula (2.46) is also U-duality invariant.

\subsubsection{Comments on brane-antibrane and other non-BPS solutions}

It should be noted that the "brane-antibrane" representation of the above nonextremal black hole is only suggestive at the moment. The subject of supergravity representation of brane-antibrane and other non-BPS systems is very much open; for a partial list of papers see $[76,77,78,79,80,81,82,83,84,85,86$. .

\subsection{Supergravity Solution with Non-zero vev of $B_{N S}$}

Our discussion so far has been devoted to supergravity solutions in which the values of all the moduli fields were set to zero. Such solutions have the characteristic that 
the mass of the D1-D5 system is a sum of the charges that characterize the system. Such bound states are marginal, without any binding energy, and can fragment into clusters of D1-D5 branes. The corresponding CFT has singularities. In order to obtain a stable bound state and a non-singular CFT we have to turn on certain moduli fields. We will consider the case when $B_{N S}$ is non-zero.

The construction of the supergravity solution that corresponds to a $\frac{1}{4}$ BPS configuration, with a non-zero $B_{N S}$ was presented in [87, 88]. $B_{N S}$ has non-zero components only along the directions $6,7,8,9$ of the internal torus. From the view point of open string theory this is then a non-commutative torus.

Here we will summarize the result. The solution contains, besides D1 and D5 brane charges, D3 brane charges that are induced by the $B_{N S}$. For simplicity we consider only non-zero values for $B_{79}$ and $B_{68}$. The asymptotic values are given by $B_{79}^{(\infty)}=b_{79}$ and $B_{68}^{(\infty)}=b_{68}$. It is important that at least 2 components of the $B_{N S}$ are non-zero, in order to be able to discuss the self-dual and anti-self-dual components.

Below we present the full solution which can be derived by a solution generating technique. Details can be found in 88.

$$
\begin{aligned}
d s^{2}= & \left(f_{1} f_{5}\right)^{-1 / 2}\left(-d t^{2}+\left(d x^{5}\right)^{2}\right)+\left(f_{1} f_{5}\right)^{1 / 2}\left(d r^{2}+r^{2} d \Omega_{3}^{2}\right) \\
& +\left(f_{1} f_{5}\right)^{1 / 2}\left\{Z_{\varphi}^{-1}\left(\left(d x^{6}\right)^{2}+\left(d x^{8}\right)^{2}\right)+Z_{\psi}^{-1}\left(\left(d x^{7}\right)^{2}+\left(d x^{9}\right)^{2}\right)\right\}, \\
e^{2 \phi}= & f_{1} f_{5} / Z_{\varphi} Z_{\psi}, \\
B_{N S}^{(2)}= & \left(Z_{\varphi}^{-1} \sin \varphi \cos \varphi\left(f_{1}-f_{5}\right)+b_{68}\right) d x^{6} \wedge d x^{8} \\
& +\left(Z_{\psi}^{-1} \sin \psi \cos \psi\left(f_{1}-f_{5}\right)+b_{79}\right) d x^{7} \wedge d x^{9}, \\
F^{(3)}= & \cos \varphi \cos \psi \tilde{K}^{(3)}+\sin \varphi \sin \psi K^{(3)}, \\
F^{(5)}= & Z_{\varphi}^{-1}\left(-f_{5} \cos \varphi \sin \psi K^{(3)}+f_{1} \cos \psi \sin \varphi \tilde{K}^{(3)}\right) \wedge d x^{6} \wedge d x^{8} \\
& +Z_{\psi}^{-1}\left(-f_{5} \cos \psi \sin \varphi K^{(3)}+f_{1} \cos \varphi \sin \psi \tilde{K}^{(3)}\right) \wedge d x^{7} \wedge d x^{9}, \\
Z_{\varphi, \psi}= & +\frac{\mu_{\varphi, \psi}}{2}\left(\frac{\alpha^{\prime}}{r^{2}}\right), \\
\mu_{\varphi}= & \mu_{1} \sin ^{2} \varphi+\mu_{5} \cos ^{2} \varphi, \mu_{\psi}=\mu_{1} \sin ^{2} \psi+\mu_{5} \cos ^{2} \psi .
\end{aligned}
$$

Here $b_{68}$ and $b_{79}$ are arbitrary constants which we have added at the end by a T-duality transformation that shifts the NS B-field by a constant. Note that for $\varphi=\psi=0$ and $b_{68}=b_{79}=0$, the above solution reduces to the known solution for D1-D5 system without B-field.

The above solution depends upon 4 parameters $\mu_{1}, \mu_{5}$, and the angles $\phi$ and $\psi$, and in general represents a system of D1, D5 and D3 branes. Since we are seeking a solution that has no source D3 branes we require that the D3 brane charges are only induced by the presence of the non-zero $B_{N S}$. This leads to certain conditions on the solutions which we do not derive here, but whose physical implication we analyze. We discuss both the asymptotically flat and near horizon geometry. 


\subsubsection{Asymptotically Flat Geometry}

In this case the induced D3 brane charges along the $(5,7,9)$ and $(5,6,8)$ directions are

$$
Q_{3}=B_{79}^{(\infty)} Q_{5}, \quad Q_{3}^{\prime}=B_{68}^{(\infty)} Q_{5},
$$

where $B_{79}^{(\infty)}=b_{79}, \quad B_{68}^{(\infty)}=b_{68}$. There is an induced contribution to the D1 brane charge. The charge $Q_{1 s}$ of the source D1 branes is

$$
Q_{1 s}=Q_{1}-b_{68} b_{79} Q_{5} .
$$

while the D5 brane charge remains unaffected by the moduli.

$\underline{\text { Mass }}$

Let us now study the mass formula as a function of the charges and the moduli. The mass corresponding to the $\frac{1}{4}$ BPS solution [46], which coincides with the ADM mass, is given in terms of the appropriate charges by

$$
M^{2}=\left(Q_{1}+Q_{5}\right)^{2}+\left(Q_{3}-Q_{3}^{\prime}\right)^{2}
$$

This can in turn be expressed in terms of $Q_{1 s}, Q_{5}$ and $b_{68}, b_{79}$

$$
M^{2}=\left(Q_{1 s}+b_{68} b_{79} Q_{5}+Q_{5}\right)^{2}+Q_{5}^{2}\left(b_{68}-b_{79}\right)^{2}
$$

We must consider the mass as a function of the moduli, holding $Q_{1 s}$ and $Q_{5}$ fixed. We see that for non-zero moduli we have a true bound state that turns marginal when the moduli are set to zero. To locate the values of the moduli which minimize the mass, we extremize the mass with respect to the moduli. The extremal values of the moduli are

$$
b_{68}=-b_{79}= \pm \sqrt{Q_{1 s} / Q_{5}-1},
$$

This says that the $B_{N S}$ moduli are self-dual, in the asymptotically flat metric. The mass at the critical point of the true bound state is then given by

$$
M^{2}=4 Q_{1 s} Q_{5}
$$

\subsubsection{Near Horizon Geometry}

In this case, absence of D3-brane sources is ensured if we set

$$
Q_{3}^{(h)}=B_{79}^{(h)} Q_{5}, \quad Q_{3}^{(h)^{\prime}}=B_{68}^{(h)} Q_{5},
$$

where

$$
\begin{aligned}
& B_{68}^{(h)}=\frac{\mu_{1}-\mu_{5}}{\mu_{\varphi}} \sin \varphi \cos \varphi+b_{68}, \\
& B_{79}^{(h)}=\frac{\mu_{1}-\mu_{5}}{\mu_{\psi}} \sin \psi \cos \psi+b_{79},
\end{aligned}
$$


are the horizon values of the two nonzero components of the B-field. Moreover, we see can that in this case

$$
\frac{B_{68}^{(h)}}{\mu_{\psi}}=-\frac{B_{79}^{(h)}}{\mu_{\varphi}},
$$

which is the self-duality condition on the B-field in the near horizon geometry. We also note that the volume of $T^{4}$ at the horizon is given by

$$
V_{T^{4}}^{(h)}=\frac{\mu_{1} \mu_{5}}{\mu_{\varphi} \mu_{\psi}}=\frac{Q_{1 s}^{(h)}}{Q_{5}} .
$$

The D1-brane charge that arises from source D1-branes in this case is given by

$$
Q_{1 s}^{(h)}=Q_{1}^{(h)}-B_{68}^{(h)} B_{79}^{(h)} Q_{5} .
$$

One can show that

$$
Q_{1 s}^{(h)}=Q_{1 s}
$$

where $Q_{1 s}$ is given by (2.60). Thus we see that not only do the parameters $b_{68}$ and $b_{79}$ have the same values here as in the asymptotically flat case, even the source D1-branes are identical, despite the total D1-brane charges being very different in the two cases.

$\underline{\text { Mass }}$

The $\frac{1}{4}$ BPS mass formula in terms of the various charge densities in this case is

$$
\left(\frac{M^{(h)}}{V_{T^{4}}^{(h)}}\right)^{2}=\left(\frac{Q_{1}^{(h)}}{V_{T^{4}}^{(h)}}+Q_{5}\right)^{2}+\left(\frac{Q_{3}^{(h)}}{\sqrt{g_{77} g_{99}}}-\frac{Q_{3}^{(h)^{\prime}}}{\sqrt{g_{66} g_{88}}}\right)^{2}
$$

Using (2.55)-(2.61) it can be easily seen that

$$
\left(M^{(h)}\right)^{2}=V_{T^{4}}^{(h)}\left(4 Q_{1 s} Q_{5}\right) .
$$

Apart from the extra factor of the $T^{4}$ volume in the near horizon geometry, this is exactly the same as (2.54). The extra volume factor correctly takes into account the difference in the 6-dimensional Newton's constant between the asymptotically flat and near horizon geometries because of the difference in the $T^{4}$ volume in the two cases. We have already seen that the B-field is automatically self-dual in the near horizon geometry and that the volume of $T^{4}$ satisfies the condition given by (2.59) and (2.60). We now see that the mass of the bound state is already at the fixed point value. Thus the solution we have here provides an explicit demonstration of the attractor mechanism [89].

The significance of this solution is that it is the description of a stable bound state in the near horizon geometry. As we shall discuss later this situation corresponds to a non-singular dual CFT. 


\subsection{Near-horizon limit and $A d S_{3} \times S^{3}$}

In this section we will exhibit the form of the classical solution in the near horizon limit of Maldacena [12]. This subsection, together with Appendix C, will be used as background for discussions of AdS/CFT correspondence in Sections 6 and 11.

The basic idea of the near horizon limit is that, near the horizon of a black hole or a black brane, the energies of particles as seen by the asymptotic observer get red-shifted:

$$
E_{\infty}=\sqrt{g_{00}} E
$$

For the metric (2.24) the red-shift factor is

$$
\sqrt{g_{00}}=\left(f_{1} f_{5}\right)^{-1 / 4}
$$

Clearly as $r \rightarrow \infty$ the red shift factor is unity. However near the horizon we get the equation

$$
E_{\infty}=\frac{r}{R} E
$$

where $R$, Eqn. (2.73), is the length scale that characterizes the geometry. In the near-horizon region, defined by

$$
r \ll R
$$

we see that the energy observed by the asymptotic observer goes to zero for finite values of E. This means that near the horizon (defined by (2.67)) an excitation of arbitrary energy looks massless. For massless modes this means that they have almost infinitely long wavelengths and for massive modes they appear as long wavelength massless excitations. If one examines the potential energy of a particle in the above geometry then in the near horizon limit the potential barrier becomes very high so that the modes near the horizon cannot get out. In the exact limit of $Q_{1}$ and $Q_{5}$ going to infinity the horizon degrees of freedom become exactly massless and decouple from the bulk degrees of freedom. As we shall see later it is in this limit that the bulk string theory is dual to a SCFT which also exhibits massless behavior in the infrared.

\subsubsection{The three-dimensional anti de Sitter space or $\mathrm{AdS}_{3}$}

We now apply these ideas to the metric of the D1-D5 black string with the KK charge $N=0$, namely (2.24).

In the region (2.67) the metric and other backgrounds are still given by (2.24), except that the harmonmic functions change to

$$
f_{1}=\frac{16 \pi^{4} g_{s} \alpha^{\prime 3} Q_{1}}{V_{4} r^{2}}, \quad f_{5}=\frac{g_{s} \alpha^{\prime} Q_{5}}{r^{2}},
$$

Here $r^{2}=x_{1}^{2}+x_{2}^{2}+x_{3}^{2}+x_{4}^{2}$ denotes the distance measured in the transverse direction to all the D-branes. The above metric differs from (2.24) only in that the harmonic functions do not have the "1" term any more (see remarks after (2.11)). 
A more precise scaling limit of the geometry is given by [90]

$$
\begin{aligned}
& \alpha^{\prime} \rightarrow 0, \frac{r}{\alpha^{\prime}} \equiv U=\text { fixed } \\
& v \equiv \frac{V_{4}}{16 \pi^{4} \alpha^{\prime 2}}=\text { fixed, } \quad g_{6}=\frac{g_{s}}{\sqrt{v}}=\text { fixed }
\end{aligned}
$$

In this limit the metric in (2.24) becomes

$$
d s^{2}=\alpha^{\prime}\left(d s_{3}^{2}+d s^{2}\left[S^{3}\right]+d s^{2}\left[T^{4}\right]\right)
$$

where

$$
d s_{3}^{2}=\left[\frac{U^{2}}{l^{2}}\left(-d x_{0}^{2}+d x_{5}^{2}\right)+l^{2} \frac{d U^{2}}{U^{2}}\right],
$$

represents three-dimensional anti-de Sitter space $\mathrm{AdS}_{3}$ (see Appendix C, Eq. (C.10) and

$$
\begin{aligned}
d s^{2}\left[S^{3}\right] & =l^{2} d \Omega_{3}^{2}, \\
d s^{2}\left[T^{4}\right] & =\sqrt{\frac{Q_{1}}{v Q_{5}}}\left(d x_{6}^{2}+\ldots d x_{9}^{2}\right),
\end{aligned}
$$

represent a three-sphere and a four-torus. Thus the near horizon geometry is that of $A d S_{3} \times S^{3} \times T^{4}$. Our notation for coordinates here is as follows: $A d S_{3}:\left(x_{0}, x_{5}, r\right)$, $S^{3}:\left(\Omega_{3}=(\chi, \theta, \phi)\right), T^{4}:\left(x_{6}, x_{7}, x_{8}, x_{9}\right) . r, \chi, \theta, \phi$ are spherical polar coordinates for the directions $x_{1}, x_{2}, x_{3}, x_{4}$. The length scale $l$ is the dimensionless radius of $S^{3}$ and the anti-de Sitter space:

$$
l=\frac{R}{\sqrt{\alpha^{\prime}}}, R=\sqrt{\alpha^{\prime}}\left(g_{6}^{2} Q_{1} Q_{5}\right)^{1 / 4} .
$$

Note that the effective string coupling in the near horizon limit is given by

$$
g_{e f f}=g_{6} \sqrt{Q_{1} / Q_{5}}
$$

The formulas for the blackhole entropy and temperature, which depend only on the near horizon properties of the geometry, do not change in the near horizon limit.

In Section 6 we will discuss in detail the symmetries of the near-horizon geometry (2.70). The spacetime symmetries as well as supersymmetries get enhanced compared to (2.25), (2.26).

\subsubsection{The BTZ black hole}

The above discussion was about the near-horizon limit of the six dimensional black string. We now turn to the near-horizon limit of the five-dimensional black hole (2.40). The near horizon scaling limit is given by [91, 90]

$$
\alpha^{\prime} \rightarrow 0, \quad r \rightarrow 0, \quad r_{0} \rightarrow 0
$$


with

$$
\begin{gathered}
U \equiv \frac{r}{\alpha^{\prime}}=\text { fixed } \quad U_{0} \equiv \frac{r_{0}}{\alpha^{\prime}}=\text { fixed } \\
v \equiv \frac{V_{4}}{16 \pi^{4} \alpha^{\prime 2}}=\text { fixed } \quad g_{6}=\frac{g_{s}}{\sqrt{v}}=\text { fixed } \quad R_{5}=\text { fixed }
\end{gathered}
$$

In this limit the metric of the D1-D5 black hole (cf. Eqn. (2.39)) reduces to the following

$$
d s^{2}=\alpha^{\prime}\left(d s_{3}^{2}+d s^{2}\left[S^{3}\right]+d s^{2}\left[T^{4}\right]\right)
$$

where the metric on the 3 -sphere and 4-torus are still given by (2.72), whereas

$$
d s_{3}^{2}=\frac{\alpha^{\prime} U^{2}}{l^{2}}\left(-d x_{0}^{2}+d x_{5}^{2}\right)+\frac{\alpha^{\prime} U_{0}^{2}}{l^{2}}\left(\cosh \sigma d t+\sinh \sigma d x_{5}^{2}\right)^{2}+\frac{\alpha^{\prime} l^{2}}{U^{2}-U_{0}^{2}} d U^{2}
$$

represents now the BTZ geometry, as we will show below. Thus the near-horizon geometry is BTZ $\times T^{4} \times S^{3}$. Our co-ordinate definitions here are as follows: $t, \phi, \tilde{r}$ refer to BTZ co-ordinates, $\Omega_{3}$ stands for the $S^{3}$ and $x_{6}, x_{7}, x_{8}, x_{9}$ stand for the co-ordinates of $T^{4}$. To identify $d s_{3}^{2}$ with the BTZ metric (i.e., the black hole in three-dimensional anti de-Sitter space discovered by [92]), we make the coordinate redefinitions given below [91, 90]

$$
\begin{aligned}
\tilde{r}^{2} & =\left(U^{2}+U_{0}^{2} \sinh ^{2} \sigma\right) \frac{R_{5}^{2}}{l^{2}} \\
r_{+} & =\frac{R_{5} U_{0} \cosh \sigma}{l} \\
r_{-} & =\frac{R_{5} U_{0} \sinh \sigma}{l} \\
\phi=\frac{x_{5}}{R_{5}}, & \quad t=\frac{l x^{0}}{R_{5}}
\end{aligned}
$$

The metric (2.77) in these new coordinates is given by

$$
d s_{3}^{2}=-\frac{\alpha^{\prime}\left(\tilde{r}^{2}-r_{+}^{2}\right)\left(\tilde{r}^{2}-r_{-}^{2}\right)}{l^{2} \tilde{r}^{2}} d t^{2}+\frac{\alpha^{\prime} \tilde{r}^{2} l^{2}}{\left(\tilde{r}^{2}-r_{+}^{2}\right)\left(\tilde{r}^{2}-r_{-}^{2}\right)} d r^{2}+\alpha^{\prime} \tilde{r}^{2}\left(d \phi+\frac{r_{+} r_{-}}{\tilde{r}^{2} l} d t\right)^{2}
$$

In this form, the metric coincides with that of a BTZ black hole (cf. Eqs. (C.11), (C.13)), with mass $M$ and angular momentum $J$ given by (cf. (C.12))

$$
M=\frac{r_{+}^{2}+r_{-}^{2}}{l^{2}}, \quad J=\frac{\sqrt{\alpha^{\prime}} 2 r_{+} r_{-}}{l}
$$

The mass $M$ and the angular momentum $J$ for the BTZ black hole are related to the parameters of the D1-D5 black hole by

$$
\frac{M}{2}=L_{0}+\bar{L}_{0}=\frac{N_{L}+N_{R}}{Q_{1} Q_{5}}, \quad \frac{J}{2 \sqrt{\alpha^{\prime} l}}=L_{0}-\bar{L}_{0}=\frac{N_{L}-N_{R}}{Q_{1} Q_{5}}
$$


where $N_{L}, N_{R}$ are defined in (2.42) and $L_{0}, \bar{L}_{0}$ are the levels of the SCFT. The extremal limit is given by $r_{+}=r_{-}$. From (2.80) and (2.81) we see that in the extremal limit $N_{R}=0$ as expected for the D1-D5 black hole.

It is important to mention the global properties of the metric (2.79), especially in relation to those of the $\mathrm{AdS}_{3}$ metric in (2.70) above. Let us consider the simplest BTZ solution first, namely with $r_{+}=r_{-}=0$. Substituting these values in (2.79) we find the metric is given by

$$
\frac{d s_{3}^{2}}{\alpha^{\prime}}=-\frac{\tilde{r}^{2}}{l^{2}} d x_{0}^{2}+\frac{l^{2}}{\tilde{r}^{2}} d r^{2}+\tilde{r}^{2} d \phi^{2}
$$

By comparison with (2.70) one can see that this metric is locally $A d S_{3}$ except for the global identification $\phi \equiv \phi+2 \pi$. This of course reflects the fact that the $r_{+}=r_{-}=0 \mathrm{BTZ}$ solution corresponds to the near-horizon limit of the D1-D5 string (with $N_{L}=N_{R}=0$ ) wrapped on a circle along $x^{5} \equiv x^{5}+2 \pi R_{5}$.

This periodic identification has two important implications:

(a) Firstly, that the zero-mass BTZ black hole is a quotient of the $\mathrm{AdS}_{3}$ space by a discrete isometry. Indeed, as has been shown in 90] the global property of the more general near-horizon solution (2.79) also corresponds to an appropriate quotient of $\mathrm{AdS}_{3}$ by a discrete isometry, consistent with the expected global properties of BTZ black holes [92].

(b) Secondly, the difference between the geometry of the zero mass $B T Z$ black hole and that of the $\mathrm{AdS}_{3}$ (although identical locally) leads to an important difference in the boundary conditions for the fermions. For the case of $\mathrm{AdS}_{3}$ the fermions are anti-periodic in $\phi$ and for the zero mass BTZ black hole they are periodic in $\phi$. One can easily see that the constant time slice of the metric in (2.71) has the topology of a disk. This forces the fermions to be anti-periodic in $\phi$ for $\mathrm{AdS}_{3}$. For the case of the zero mass BTZ black hole in (2.82), the constant time slice has a singularity at $\tilde{r}=0$. Therefore the fermions can be both periodic or anti-periodic. An analysis of the Killing spinors in the background of the zero mass BTZ black hole shows that the fermions in fact have to be periodic [93].

\subsubsection{The two-dimensional black hole}

In this subsubsection we will discuss the near-horizon geometry of a related (pure 5-brane) system and its connection to the two dimensional black hole [94, 95, 96].

Consider the supergravity solution of the non-extremal black hole in type IIB string theory (2.40) with $Q_{1}=0$ and $\sigma=0$. The D1-D5 supergravity solution then reduces to the non-extremal D5-brane. We will now show that there is a near horizon region where the geometry can be approximated to that of two dimensional black hole [94, 95, 96]. The ten dimensional geometry with $Q_{1}=0$ is given by

$$
e^{-2 \phi}=\frac{1}{g_{s}^{2}}\left(1+\frac{r_{5}^{2}}{r^{2}}\right)
$$




$$
\begin{aligned}
H_{\theta \phi \psi} & =Q_{5} \alpha^{\prime} \\
d s^{2} & =\left(1+\frac{g_{s} Q_{5} \alpha^{\prime}}{r^{2}}\right)^{-\frac{1}{2}}\left(-\left(1-\frac{r_{0}^{2}}{r^{2}}\right) d t^{2}+d x_{5}^{2}+\cdots d x_{9}^{2}\right) \\
& +\left(1+\frac{g_{s} Q_{5} \alpha^{\prime}}{r^{2}}\right)\left(\frac{d r^{2}}{1-\frac{r_{0}}{r^{2}}} d r^{2}+r^{2} d \Omega_{3}^{2}\right)
\end{aligned}
$$

To obtain the near horizon geometry We use the IMSY limit 97 for the case of D5 branes. This is given by

$$
\begin{array}{r}
U=\frac{r}{\alpha^{\prime}}=\text { fixed, } \quad U_{0}=\frac{r_{0}}{\alpha^{\prime}}=\text { fixed }, \\
g_{\mathrm{YM}}^{2}=(2 \pi)^{3} g_{s} \alpha^{\prime}=\text { fixed } \\
\text { with } \alpha^{\prime} \rightarrow 0
\end{array}
$$

Here $g_{\mathrm{YM}}$ is the Yang-Mills coupling on the D5-brane. This geometry is nonconformal and the dilaton depends on the scale $U$. For $\sqrt{Q_{5}} \ll g_{\mathrm{YM}} U$, the string coupling and the curvature in string units is large, and therefore the valid discription of the background is obtained by performing an S-duality. The solution reduces to the near horizon geometry of non-extremal NS 5-branes. The metric and the dilaton after S-duality is given by

$$
\begin{aligned}
e^{2 \phi} & =\frac{(2 \pi)^{3} Q_{5}}{g_{\mathrm{YM}}^{2} U^{2}} \\
d s^{2} & =-\left(1-\frac{U_{0}^{2}}{U^{2}}\right) d t^{2}+\left(d x_{5}^{2}+\cdots d x_{9}^{2}\right)+g_{s} \alpha^{\prime} Q_{5}\left(\frac{d U^{2}}{U^{2}\left(1-\frac{U_{0}}{U}\right)}+d \Omega_{3}^{2}\right)
\end{aligned}
$$

Here we have scaled the metric by $g_{s}$ so that the 10 dimenional Newton's constant is invariant. To see this is the metric of the (2d black hole $) \times S^{3} \times R^{5}$ we change coordinates by substituting $U=U_{0} \cosh \gamma$. Then we get

$$
\begin{aligned}
e^{2 \phi} & =\frac{(2 \pi)^{3} Q_{5}}{g_{\mathrm{YM}}^{2} U_{0}^{2}} \frac{1}{\cosh ^{2} \gamma} \\
d s^{2} & =-\tanh ^{2} \gamma d t^{2}+g_{s} \alpha^{\prime} Q_{5}\left(d \gamma^{2}+d \Omega_{3}^{2}\right)+\left(d x_{5}^{6}+\cdots d x_{9}^{2}\right)
\end{aligned}
$$

Now it is easily seen that the geometry reduces to that of ( $2 \mathrm{~d}$ black hole $) \times S^{3} \times R^{5}$. This near horizon limit of extremal NS 5-branes was obtained in 98. 


\section{Semi-classical derivation of Hawking radiation}

We described in some detail the construction of the D1-D5 black hole, (2.27), (2.40), in the last section. We will now address the issue of absorption and Hawking radiation by this black hole. Both absorption and Hawking radiation involve interesting questions, as we remarked in the introduction. For instance, classically the black hole only absorbs and does not emit. One of our goals will be to ultimately interpret this in the microscopic model, explaining thereby a crucial aspect of the event horizon. Secondly, the semiclassical treatment of Hawking radiation leads to the information puzzle, and we would like to see how standard scattering processes described in terms of the microscopic model gives rise to such a radiation within a unitary quantum theory.

Before we proceed to the microscopic description, however, we will devote the present section to briefly review the (semi)classical calculations of absorption/emission of particles (in the type IIB spectrum) by the D1-D5 black hole (2.40).

The absorption cross-section and emission rate of a particular field depend on how the field propagates and backscatters from the geometry of the black hole. We will look at the equation of propagation of scalar fluctuations.

We begin by writing down the IIB Lagrangian [99, 100, 101] compactified on $T^{5}$ (of which the D1-D5 black hole (2.40), (2.41) is a solution):

$$
\begin{aligned}
S_{5}= & \frac{1}{2 \kappa_{5}^{2}} \int d^{5} x \sqrt{-g}\left[R-\frac{4}{3}\left(\partial_{\mu} \phi_{5}\right)^{2}-\frac{1}{4} G^{a b} G^{c d}\left(\partial_{\mu} G_{a c} \partial^{\mu} G_{b d}+e^{2 \phi_{5}} \sqrt{G} \partial_{\mu} C_{a c} \partial^{\mu} C_{b d}\right)\right. \\
& \left.-\frac{e^{-4 \phi_{5} / 3}}{4} G_{a b} F_{\mu \nu}^{a} F^{b \mu \nu}-\frac{e^{2 \phi_{5} / 3}}{4} \sqrt{G} G^{a b} H_{\mu \nu a} H_{b}^{\mu \nu}-\frac{e^{\left(4 \phi_{5} / 3\right)}}{12} \sqrt{G} H_{\mu \nu \lambda}^{2}\right]
\end{aligned}
$$

Notation: $a, b, \ldots=5, \ldots, 9$ denote the directions along $T^{5}$, while $\mu, \nu, \ldots=$ $0, \ldots, 4$ denote the non-compact directions. We have included in the above Lagrangian only the following ten-dimensional fields:

- the ten dimensional dilaton $\phi$,

- the ten-dimensional string-frame metric $d s^{2} \quad{ }^{6}$ written as

$$
d s^{2}=g_{\mu \nu} d x^{\mu} d x^{\nu}+G_{a b}\left(d y^{a}+A_{\mu}^{a} d x^{\mu}\right)\left(d y^{b}+A_{\nu}^{b} d x^{\nu}\right)
$$

which identifies $A_{\mu}^{a}$ as the KK vector fields,

- and the RR 2-form field $C^{(2)}$ written as

$$
C^{(2)}=C_{\mu \nu} d x^{\mu} \wedge d x^{\nu}+C_{a b} d x^{a} \wedge d x^{b}
$$

\footnotetext{
${ }^{6}$ related to the Einstein frame metric $d s_{E}^{2}$ as $d s^{2}=\exp [\phi / 2] d s_{E}^{2}$
} 
The various fields appearing in (3.1) are defined in terms of the above fields, as follows

- the five-dimensional dilaton $\phi_{5}=\phi_{10}-(1 / 4) \ln \left[\operatorname{det}_{a b} G_{a b}\right]$,

- the KK field strengths $F^{a}=d A^{a}$,

- and the $H$-fields given by 99

$$
\begin{aligned}
& H_{\mu \nu a}=\mathrm{F}_{a \mu \nu}-C_{a b} F_{\mu \nu}^{b} \\
& H_{\mu \nu \lambda}=\partial_{\mu} \mathrm{B}_{\nu \lambda}-\frac{1}{2} A_{\mu}^{a} \mathrm{~F}_{a \nu \lambda}-\frac{1}{2} \mathrm{~A}_{a \mu} F_{\nu \lambda}^{a}+\text { cyc.perm. }
\end{aligned}
$$

where

$$
\mathrm{A}_{a \mu}=C_{\mu a}+C_{a b} A_{\mu}^{b}, \mathrm{~F}_{a}=d \mathrm{~A}_{a}, \mathrm{~B}_{\mu \nu}=C_{\mu \nu}+A_{[\mu}^{a} \mathrm{~A}_{\nu] a}-A_{\mu}^{a} C_{a b} A_{\nu}^{b}
$$

The five-dimensional Newton's constant $16 \pi\left(G_{N}^{5}\right)^{2} \equiv 2 \kappa_{5}^{2}$ is defined as in (2.29).

We will now simplify the Lagrangian even further, by assuming that (a) of the KK-gauge fields only $A_{\mu}^{5}$ is non-zero and is of the "electric" type (b) $C_{a b}=0$. This is a consistent truncation, and D1-D5 black hole (2.49) is a solution of the truncated system.

We will consider below two separate sets of scalar fluctuations:

1. This set of fluctuations $h_{a b}, a \neq b, a, b=6,7,8,9$ are defined by

$$
G_{a b}=f_{1}^{1 / 2} f_{5}^{-1 / 2}\left(\delta_{a b}+h_{a b}\right), a, b=6,7,8,9
$$

Recall that $\left\langle G_{a b}\right\rangle=f_{1}^{1 / 2} f_{5}^{-1 / 2} \delta_{a b}$ represents the background value (cf. (2.39)).

2. This set of fluctuations are defined by

$$
\begin{aligned}
& \text { - } e^{2 \nu} \equiv G_{66} \text { (assumed equal to } G_{77}=G_{88}=G_{99} \text { ), } \\
& \text { - } \phi \equiv \phi_{5}+\frac{1}{2} \nu_{5} \text {, where } e^{2 \nu_{5}}=G_{55}, \\
& \text { - } \lambda \equiv \frac{3}{4} \nu_{5}-\frac{1}{2} \phi_{5} \text {. }
\end{aligned}
$$

For the fluctuations in Case 1, the action (3.1) reduces to the following action

$$
S=-\frac{1}{8 \kappa_{5}^{2}} \int d^{5} x \sqrt{-g} \partial_{\mu} h_{a b} \partial^{\mu} h_{a b}
$$

whereas for the three fluctuations in Case 2, the action (3.1) reduces to

$$
\begin{aligned}
S & =\frac{1}{2 \kappa_{5}^{2}} \int d^{5} x \sqrt{-g}\left[R-\left(\partial_{\mu} \phi\right)^{2}\right. \\
& \left.-\frac{4}{3}\left(\partial_{\mu} \lambda\right)^{2}-4\left(\partial_{\mu} \nu\right)^{2}-\frac{1}{4} e^{\frac{8}{3} \lambda}\left(F_{\mu \nu}^{5}\right)^{2}-\frac{1}{4} e^{-4 / 3 \lambda+4 \nu} \mathrm{F}_{5, \mu \nu}^{2}-\frac{1}{12} e^{4 / 3 \lambda+4 \nu} H_{\mu \nu \lambda}^{2}\right](3.5)
\end{aligned}
$$


The background values of the 5-D Einstein metric $g_{\mu \nu}$ and the other fields are to be read off from the D1-D5 black hole solution (2.40), (2.41).

Note that $h_{a b}, a \neq b$ and $\phi$ couple only to the $g_{\mu \nu}$ (see Eqn. (2.40)); because of this property they are called "minimal scalars". On the other hand, $\nu, \lambda$ couple to the dilaton and the RR fields as well; these are called "fixed scalars" because their value at the horizon cannot be arbitrarily chosen but are fixed by the charges $Q_{1}, Q_{5}, N$.

In the following we will first calculate the absorption crosssection for minimal scalars, and later briefly mention the case of fixed scalars.

\subsection{Minimal scalar}

For the semiclassical absorption/emission [38, 39, 40], all we need is the equation for propagation of the fluctuation $h_{a b}$ (or $\phi$ ) on the black hole metric $g_{\mu \nu}$. We will denote the minimal scalar fluctuation generically by the symbol $\varphi$; since it couples only to the five-dimensional Einstein metric (3.4), the equation of motion is

$$
D_{\mu} \partial^{\mu} \varphi=0
$$

For the five-dimensional black hole metric (2.40) the above equation becomes for the s-wave mode:

$$
\left[\frac{h}{r^{3}} \frac{d}{d r}\left(h r^{3} \frac{d}{d r}\right)+f w^{2}\right] R_{w}(r)=0
$$

where $\varphi=R_{w}(r) \exp [-i w t]$.

The idea behind the absorption calculation is very simple. In terms of $\psi=$ $r^{3 / 2} R$ the above equation becomes

$$
\left[-\frac{d^{2}}{d r_{*}^{2}}+V_{w}\left(r_{*}\right)\right] \psi=0
$$

where

$$
V_{w}\left(r_{*}\right)=-w^{2} f+\frac{3}{4 r^{2}}\left(1+2 r_{0}^{2} / r^{2}-3 r_{0}^{4} / r^{4}\right)
$$

The shape of the potential is given by (Fig 2). Absorption is caused by the tunnelling of an incoming wave into the "pit of the potential".

\section{Near and Far solutions:}

It is not possible to solve the wave equation exactly. However, we can devise near and far zones where the potential simplifies enough to admit known solutions. If the zones have an overlap region then matching the near and far wave-functions and their radial derivatives will provide the solution for our purpose. In the following we will closely follow [40]. We will work in the following range of frequency and parameters

$$
\begin{aligned}
r_{0}, r_{n} & \ll r_{1}, r_{5} \\
w r_{5} & \ll 1
\end{aligned}
$$




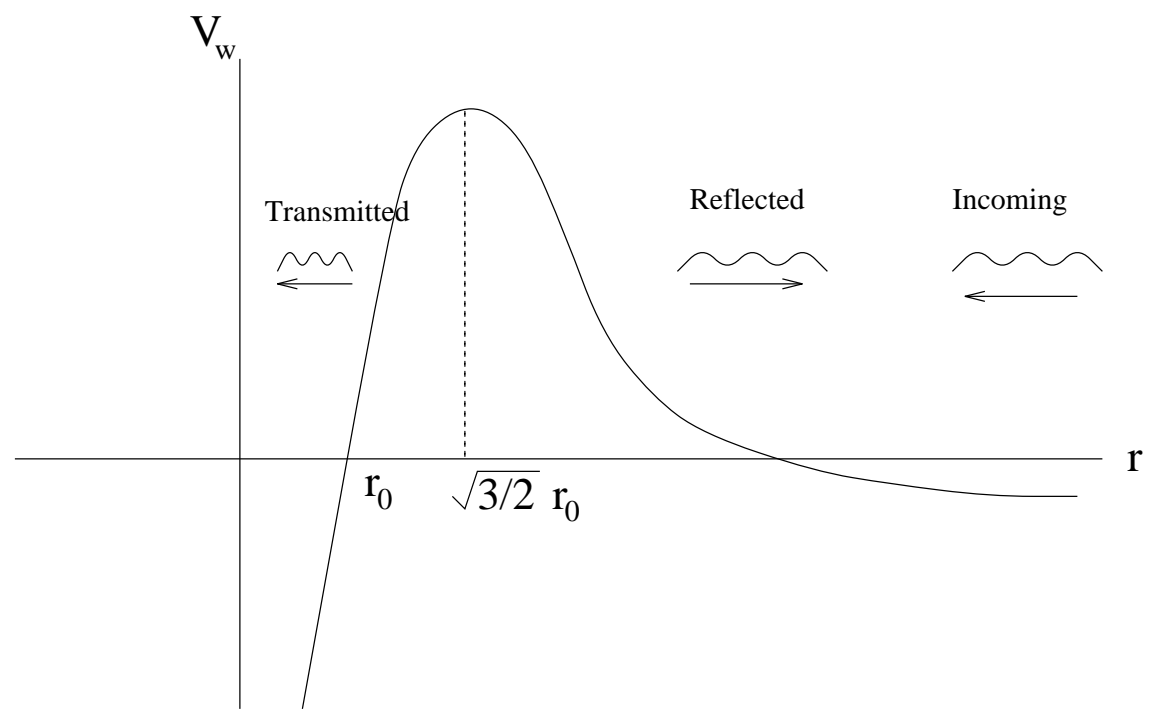

Figure 2: Potential for minimal scalar

The far and near solutions will be matched at an intermediate point $r_{m}$ such that

$$
r_{0}, r_{n} \ll r_{m} \ll r_{1}, r_{5}, \quad w r_{1} \ll r_{m} / r_{1}
$$

The existence of such an intermediate point $r_{m}$ is guaranteed by (3.9).

Far zone $\left(r>r_{m}\right)$ :

Here the potential $V_{w}$ becomes (in terms of $\rho=w r$ )

$$
V_{w}(\rho)=-w^{2}\left(1-\frac{3}{4 \rho^{2}}\right)
$$

This gives a Bessel equation, so that

$$
\begin{aligned}
\psi & =\alpha F(\rho)+\beta G(\rho) \\
F(\rho) & =\sqrt{\pi \rho / 2} J_{1}(\rho), \quad G(\rho)=\sqrt{\pi \rho / 2} N_{1}(\rho)
\end{aligned}
$$

Using $R=r^{-3 / 2} \psi$ and the asymptotic forms of the above Bessel functions, we find the following asymptotic form for $R$ :

$$
R=\frac{1}{r^{3 / 2}}\left[\frac{e^{i w r}}{2}\left(\alpha e^{-i 3 \pi / 4}-\beta e^{-i \pi / 4}\right)+\frac{e^{-i w r}}{2}\left(\alpha e^{i 3 \pi / 4}-\beta e^{i \pi / 4}\right)\right]
$$

Near zone $\left(r<r_{m}\right)$ :

Here we have

$$
\frac{h}{r^{3}} \frac{d}{d r}\left(h r^{3} \frac{d}{d r} R\right)+\left[\frac{\left(w r_{n} r_{1} r_{5}\right)^{2}}{r^{6}}+\frac{w^{2} r_{1}^{2} r_{5}^{2}}{r^{4}}\right] R_{w}(r)=0
$$


which is a Hypergeometric equation, with solution 40]

$$
\begin{aligned}
R & =A \tilde{F}+B \tilde{G} \\
\tilde{F} & =z^{-i(a+b) / 2} F(-i a,-i b, 1-i a-i b, z) \\
\tilde{G} & =z^{i(a+b) / 2} F(-i a,-i b, 1-i a-i b, z) \\
z & =\left(1-r_{0}^{2} / r^{2}\right), a=w /\left(4 \pi T_{R}\right), b=w /\left(4 \pi T_{L}\right)
\end{aligned}
$$

where we have introduced two parameters $T_{L}, T_{R}$, given by

$$
T_{L, R}=\frac{r_{0}}{2 \pi r_{1} r_{5}} e^{ \pm \alpha_{n}}=\frac{1}{2 \pi r_{0} \sinh \left(\alpha_{1}\right) \sinh \left(\alpha_{5}\right) \exp \left[\mp \alpha_{n}\right]}
$$

These, as will see in Section 5, play the role of 'left-' and 'right'-moving temperatures (cf. Eqn. (4.29)). In the second step we have used the definition of the gravitational lengths $r_{1}, r_{5}$ in (2.40).

We now impose on the "near solution" (3.15) the condition that the wave at the horizon should not have any outcoming component: it should be purely ingoing (no "white hole"). This gives $B=0$.

\section{Matching}

We now match $R$ and $d R / d r$ between the near and far regions at some point $r_{m}$ in the overlap region. This gives

$$
\sqrt{\pi / 2} w^{3 / 2} \alpha / 2=A \frac{\Gamma(1-i a-i b)}{\Gamma(1-i b) \Gamma(1-i a)}, \quad \beta / \alpha \ll 1
$$

\section{$\underline{\text { Fluxes }}$}

The equation (3.6) for $R$ implies $\frac{d}{d r} \mathcal{F}=0$, where

$$
\mathcal{F}(r)=\frac{1}{2 i}\left[R^{*} h r^{3} d R / d r-\text { c.c. }\right]
$$

In order to find out what fraction of the flux gets absorbed at the horizon, we compute the ratio

$$
R_{1}=\mathcal{F}\left(r_{0}\right) / \mathcal{F}^{i n}(\infty)=r_{0}^{2} \frac{a+b}{w\left|e_{1}\right|^{2}} w^{3} \pi / 2
$$

where the superscript "in" indicates the flux calculated from the "ingoing" part of the wave at infinity.

\subsubsection{Absorption Cross-section:}

In order to define absorption cross-section in the standard way, one has to consider plane waves and not $s$-waves. It is easy to derive that

$$
e^{-i w z}=\left(4 \pi / w^{3}\right) e^{-i w r} Z_{000}+\text { other partial waves }
$$


where we use the notation $Z_{l m_{1} m_{2}}$ for the $S_{3}$ analogs of the spherical harmonics $Y_{l m}$. From this and the standard definition of absorption cross-section we get

$$
\sigma_{a b s}=\left(4 \pi / w^{3}\right) R_{1}
$$

which evaluates to [40]

$$
\sigma_{a b s}=2 \pi^{2} r_{1}^{2} r_{5}^{2} \frac{\pi w}{2} \frac{\exp \left(w / T_{H}\right)-1}{\left(\exp \left(w / 2 T_{R}\right)-1\right)\left(\exp \left(w / 2 T_{L}\right)-1\right)}
$$

where $T_{L, R}$ is given by (3.16), and $T_{H}$, to be identified below with the Hawking temperature, is given by the harmonic mean

$$
\frac{1}{T_{H}}=\frac{1}{2}\left(\frac{1}{T_{L}}+\frac{1}{T_{R}}\right)=2 \pi r_{0} \sinh \left(\alpha_{1}\right) \sinh \left(\alpha_{5}\right) \cosh \left(\alpha_{n}\right)
$$

Note that in the regime (3.9), the Hawking temperature agrees with (2.45). We will make this comparison in Section 4.6 where we will also compare the temperatures (3.16), (3.23) with the values obtained from the D1-D5 CFT (see also Section 8).

In the $w \rightarrow 0$ limit, one gets [38]

$$
\sigma_{a b s}=A_{h}
$$

where $A_{h}$ denotes the area of the event horizon.

\subsubsection{Hawking radiation:}

The semiclassical calculation of Hawking radiation is performed through the standard route of finding Bogoliubov coefficients representing mixing of negative and positive frequency modes due to evolution from "in" to "out" vacua, defined with respect to Minkowski observers existing in the asymptotically flat regions at $t=-\infty$ and $t=+\infty$ respectively [16] (see, e.g. [18, 102 for more leisurely derivations).

The rate of radiation is given by

$$
\Gamma_{H}=\sigma_{a b s}\left(e^{w / T_{H}}-1\right)^{-1} \frac{d^{4} k}{(2 \pi)^{4}}
$$

As we remarked above, the Hawking temperature, given by Eqns. (3.23), agrees with the temperature (2.45) in the region (3.9) (see Section 4.6).

We will see in Section 8 how $\Gamma_{H}$ and $\sigma_{a b s}$ are reproduced in the D-brane picture.

\subsubsection{Importance of near-horizon physics}

It is interesting to note two points for later use:

(a) The "near zone" described above is simply the near-horizon region as in the AdS/CFT context (see Sections 6 and 8), 
(b) With the inequality $\beta \ll \alpha$ in (3.17) the solution (3.13) in the Far zone simply becomes

$$
R=\frac{\alpha}{\sqrt{2}} e^{i w r}
$$

which is just a free incoming wave, with flux $\mathcal{F}^{i n}(\infty)=|\alpha|^{2}$. As we saw (Eqn. (3.19) ), the parameter $\alpha$ also disappears from the ultimate calculation because of the division by the flux at infinity.

Thus, at the end of the day, it is only the near-horizon geometry, together with the mere existence of the asymptotically flat region, which ultimately determines the absorption cross-section (3.22) and the Hawking flux (3.25).

\subsection{Fixed scalars}

The graybody factor for the fixed scalars phi, $\lambda$ [101, 103, 104, 105, 106] (also reviewed in [107]) follows from a similar, but more involved, analysis of the (coupled) equations of motion of these two fields which follow from the action (3.5). These were solved for general $Q_{1}, Q_{5}, N$ in [104]. The method for computing the absorption cross-section and the Hawking rate is similar to those employed for the minimal scalars. As we noted above, the important ingredient in the semiclassical calculation is the near-horizon equation of motion; this turns out be Eq. (8.57). Using this, we arrive at the following result for the absorption cross-section for fixed scalars (for $w, T_{R} \ll T_{L}$ )

$$
\sigma_{a b s}=\frac{1}{4} A_{h}\left(w r_{n}\right)^{2}\left(1+\frac{4 \pi^{2} T_{H}^{2}}{w^{2}}\right)
$$

where the temperatures $T_{L}, T_{R}, T_{H}$ are defined in (3.16), (3.23), and the area $A_{h}$ of the horizon is defined in (2.44).

As we will see in Section 8, understanding the absorption and emission of fixed scalars from D-brane models is a subtle problem[104]. Resolution of this problem requires [108] a new insight from AdS/CFT correspondence about coupling of Dbranes to supergravity. 


\section{The microscopic modeling of black hole and gauge theory of the D1-D5 system}

In section 2.2 we discussed the supergrativy solution of the D1-D5 black string solution. The solution with $N=0$, then it it consists of $Q_{1}$ D1-branes and $Q_{5}$ D5-branes. The realization that solitons carrying Ramond-Ramond charges can be represented at weak string coupling by open strings with Dirichlet boundary conditions [109] allows the formulation of the microscopic theory for the D1-D5 system. We will be interested in only low energy degrees of freedom of the D1-D5 system, and thus we ignore all the massive string modes. There are two ways to proceed in the study of the massless modes, and we shall discuss both of them. The first method is a description in terms of a 2-dimensional gauge theory of the D-branes and the second method involves identifying D1 branes with instantons of a 4 dimensional gauge theory. The latter description is more accurate and is valid for instantons of all sizes. The 2-dimensional gauge theory description is valid near the point in the moduli space of instantons when the instantons have shrunk to zero size [110]. We will discuss this more approximate description first and detail the domain of validity of this description.

\subsection{The D1-D5 System and the $\mathcal{N}=4, U\left(Q_{1}\right) \times U\left(Q_{5}\right)$ gauge theory in 2-dimensions}

Consider type IIB string theory with five coordinates, say $x^{5} \cdots x^{9}$, compactified on $S^{1} \times T^{4}$. The microscopic model for the solution (2.27) with $N=0$ consists $Q_{1}$ D1-branes and $Q_{5}$ D5-branes [71, 44]. The D1-branes are along the $x^{5}$ coordinate compactified to a circle $S^{1}$ of radius $R_{5} \equiv R$, while the D5-branes are parallel to $x^{5}$ and $x^{6}, \cdots, x^{9}$ compactified on a torus $T^{4}$ of volume $V_{T^{4}} \equiv V_{4}$. The charge $N$ is related to the momenta of the excitations of this system along $S^{1}$. We will work in the following region of parameter space:

$$
\begin{aligned}
& V_{4} \sim O\left(\alpha^{\prime 2}\right) \\
& R \gg l_{s} \equiv \sqrt{\alpha^{\prime}}
\end{aligned}
$$

Let us briefly discuss the implications of the above region in parameter space. The size of the torus $T^{4}$ is of the order of string scale the masses of the winding and momentum modes of the strings are of order of $1 / l_{s}$. This implies that for energies $E \ll 1 / l_{s}$ we can neglect these modes. On the other hand the radius of the $S^{1}$ is much larger than string scale. Therefore for $E \ll 1 / l_{s}$ the winding modes can be neglected but one has to retain all the momentum modes. Effectively we can then treat the circle as non-compact. We now discuss the symmetries preserved by this configuration of D-branes. The $S O(1,9)$ symmetry of 10 dimensions is broken down to $S O(1,1) \times S O(4)_{E} \times S O(4)_{I}$. The $S O(4)_{E}$ stands for rotations 


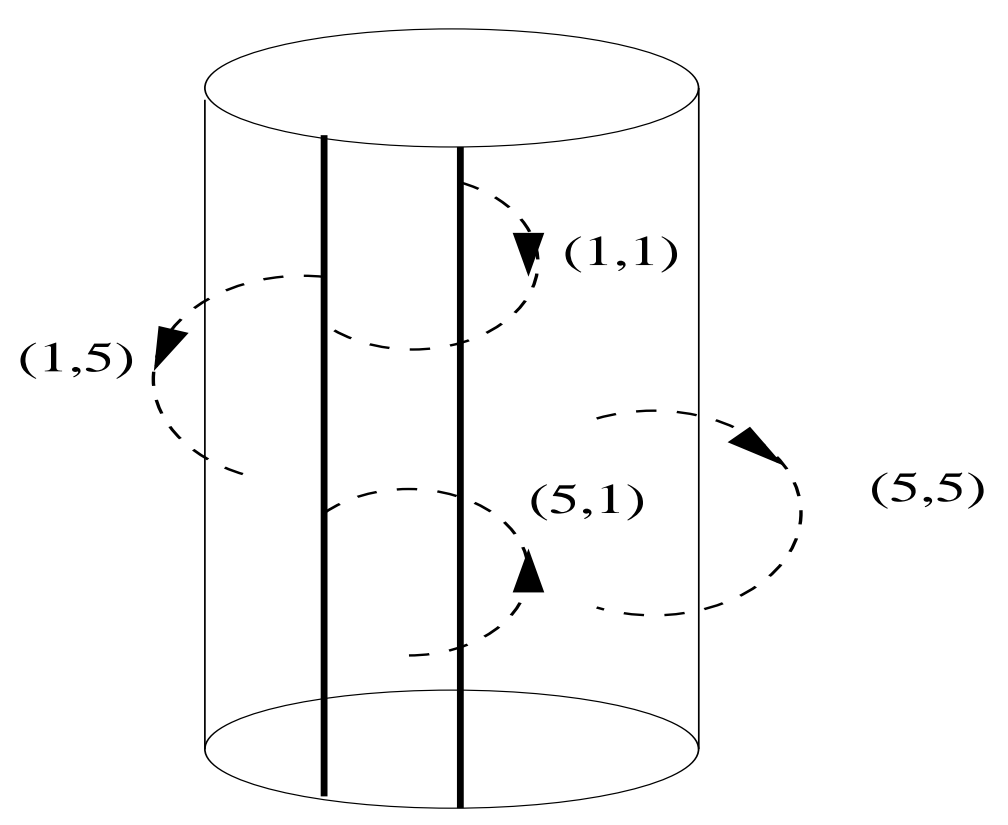

Figure 3: Open strings in the D1-D5 system

of the $6,7,8,9$ directions. As the 6,7,8,9 directions are compactified on the torus $T^{4}$, the $S O(4)_{I}$ symmetry is also broken. But we can still use the $S O(4)_{I}$ algebra to classify states and organize fields. This configuration of D-branes preserves 8 supersymmetries out of the 32 supersymmetries of type IIB theory. From the fact that we are retaining only momentum modes along the $x^{5}$ the low energy effective action for the collective modes of this D-brane configuration is $1+1$ dimensional.

More precisely, we shall see that the low-energy dynamics of this D-brane system is described by a $U\left(Q_{1}\right) \times U\left(Q_{5}\right)$ gauge theory in two dimensions with $\mathcal{N}=4$ supersymmetry [48, 111]. The gauge theory will be assumed to be in the Higgs phase because we are interested in the bound state where the branes are not separated from each other in the transverse direction. In order to really achieve this and prevent branes from splitting off we will turn on the Fayet-Iliopoulos parameters. We shall show in section 6.2 that in supergravity these parameters correspond to the vev of the Neveu-Schwarz $B_{N S}$. In principle we can also turn on the $\theta$ term in the gauge theory. This corresponds to a vev of a certain linear combination of the RR 0 -form and 4 -form.

The elementary excitations of the D-brane system (see Figure 3) correspond to open strings with two ends attached to the branes and there are three classes of such strings: the $(1,1),(5,5)$ and $(1,5)$ strings. The associated fields fall into vector multiplets and hypermultiplets, using the terminology of $\mathcal{N}=2, D=4$ supersymmetry.

\section{$\underline{(1,1) \text { strings }}$}


The part of the spectrum coming from $(1,1)$ strings is simply the dimensional reduction, to $1+1$ dimensions (the $\left(t, x^{5}\right)$-space), of the $\mathcal{N}=1, U\left(Q_{1}\right)$ gauge theory in $9+1$ dimensions 109].

The bosonic fields of this theory can be organized into the vector multiplet and the hypermultiplet of $\mathcal{N}=2$ theory in four-dimensions as

$$
\begin{array}{r}
\text { Vector multiplet: } A_{0}^{(1)}, A_{5}^{(1)}, Y_{m}^{(1)}, m=1,2,3,4 \\
\text { Hypermultiplet: } Y_{i}^{(1)}, i=6,7,8,9
\end{array}
$$

The $A_{0}^{(1)}, A_{5}^{(1)}$ are the $U\left(Q_{1}\right)$ gauge fields in the non-compact directions. The $Y_{m}^{(1)}$ 's and $Y_{i}^{(1)}$ 's are gauge fields in the compact directions of the $\mathcal{N}=1$ super YangMills in ten-dimensions. They are hermitian $Q_{1} \times Q_{1}$ matrices transforming as adjoints of $U\left(Q_{1}\right)$. The hypermultiplet of $\mathcal{N}=2$ supersymmetry are doublets of the $S U(2)_{R}$ symmetry of the theory. The adjoint matrices $Y_{i}^{(1)}$ 's can be arranged as doublets under $S U(2)_{R}$ as

$$
N^{(1)}=\left(\begin{array}{c}
N_{1}^{(1)} \\
N_{2}^{(1) \dagger}
\end{array}\right)=\left(\begin{array}{c}
Y_{9}^{(1)}+i Y_{8}^{(1)} \\
Y_{7}^{(1)}-i Y_{6}^{(1)}
\end{array}\right)
$$

\section{(5,5) strings}

The field content of these massless open strings is similar to the the $(1,1)$ strings except for the fact that the gauge group is $U\left(Q_{5}\right)$ instead of $U\left(Q_{1}\right)$. Normally one would have expected the gauge theory of the $(5,5)$ strings to be a dimensional reduction of $\mathcal{N}=1 U\left(Q_{5}\right)$ super Yang-Mills to $5+1$ dimensions. In the region (4.1) where we are working, we can ignore the Kaluza-Klein modes on $T^{4}$, effectively leading to a theory in $1+1$ dimensions. The vector multiplets and the hypermultiplets are given by

$$
\begin{array}{r}
\text { Vector multiplet: } A_{0}^{(5)}, A_{5}^{(5)}, Y_{m}^{(5)} m=1,2,3,4 \\
\text { Hypermultiplet: } Y_{i}^{(5)} i=6,7,8,9
\end{array}
$$

The $A_{0}^{(5)}, A_{5}^{(5)}$ are the $U\left(Q_{5}\right)$ gauge fields in the non-compact directions. The $Y_{m}^{(5)}$ 's and $Y_{i}^{(5)}$ 's are gauge fields in the compact directions of the $\mathcal{N}=1$ super Yang-Mills in ten-dimensions. They are hermitian $Q_{5} \times Q_{5}$ matrices transforming as adjoints of $U\left(Q_{5}\right)$. The hypermultiplets $Y_{i}^{(5)}$ 's can be arranged as doublets under $S U(2)_{R}$ as

$$
N^{(5)}=\left(\begin{array}{c}
N_{1}^{(5)} \\
N_{2}^{(5) \dagger}
\end{array}\right)=\left(\begin{array}{c}
Y_{9}^{(5)}+i Y_{8}^{(5)} \\
Y_{7}^{(5)}-i Y_{6}^{(5)}
\end{array}\right)
$$

Since $x^{m}$ are compact, the $(1,1)$ strings can also have winding modes around the $T^{4}$. These are, however, massive states in the $(1+1)$-dimensional theory and can be ignored. This is because their masses are proportional to $1 / \sqrt{\alpha^{\prime}}$ (see (4.1)), 
which can be neglected for energies $E \ll 1 / l_{s}$. Similarly, the part of the spectrum coming from $(5,5)$ strings is the dimensional reduction, to $5+1$ dimensions, of the $\mathcal{N}=1, U\left(Q_{5}\right)$ gauge theory in $9+1$ dimensions. In this case, the gauge field components $A_{m}^{(5)}(m=6,7,8,9)$ also have a dependence on $x^{m}$. Momentum modes corresponding to this dependence are neglected because the size of the 4-torus is of the order of the string scale $\sqrt{\alpha^{\prime}}$. The neglect of the winding modes of the $(1,1)$ strings and the KK modes of the $(5,5)$ strings is consistent with T-duality. A set of four T-duality transformations along $x^{m}$ interchanges D1- and D5-branes and also converts the momentum modes of the $(5,5)$ strings along $T^{4}$ into winding modes of $(1,1)$ strings around the dual torus [112]. Since these winding modes have been ignored, a T-duality covariant formulation requires that we should also ignore the associated momentum modes.

\section{$\underline{(1,5) \text { and }(5,1) \text { strings }}$}

The field content obtained so far is that of $\mathcal{N}=2, U\left(Q_{1}\right) \times U\left(Q_{5}\right)$ gauge theory, in $1+5$ dimensions, reduced to $1+1$ dimensions on $T^{4}$.

The $S O(4)_{I} \sim S U(2)_{L} \times S U(2)_{R}$ rotations on the tangent space of the torus act on the components of the adjoint hypermultiplets $X_{i}^{(1,5)}$ as an $R$-symmetry. To this set of fields we have to add the fields from the $(1,5)$ sector that are constrained to live in $1+1$ dimensions by the ND boundary conditions. These strings have their ends fixed on different types of D-branes and, therefore, the corresponding fields transform in the fundamental representation of both $U\left(Q_{1}\right)$ and $U\left(Q_{5}\right)$. The ND boundary conditions have the important consequence that the $(1,5)$ sector fields form a hypermultiplet which is chiral w.r.t. $S O(4)_{I}$. The chirality projection is due to the GSO projection. Hence the $R$-symmetry group is $S U(2)_{R}$.

$$
\chi=\left(\begin{array}{c}
A \\
B^{\dagger}
\end{array}\right)
$$

A few comments are in order:

1. The inclusion of these fields, coming from the $(1,5)$ and $(5,1)$ strings, breaks the supersymmetry by half, to the equivalent of $\mathcal{N}=1$ in $D=6$, and the final theory only has $S U(2)_{R} R$-symmetry.

2. The fermionic superpartners of these hypermultiplets which arise from the Ramond sector of the massless excitations of $(1,5)$ and $(5,1)$ strings carry spinorial indices under $S O(4)_{E}$ and they are singlets under $S O(4)_{I}$.

3. The $U(1) \times \overline{U(1)}$ subgroup is important. One combination involving the sum of the $U(1)$ 's leaves the hypermultiplet invariant. $\left(A_{a^{\prime} a}, B_{a^{\prime} a}\right)$ have charges $(+1,-1)$ under the relative $U(1)$.

4. $\chi$ is a chiral spinor of $S O(4)_{I}$ with convention $\Gamma_{6789} \chi=\chi$. 
5. Since we are describing the Higgs phase in which all the branes sit on top of each other we have $Y_{i}^{(1,5)}=0$.

6. There are two coupling constants in the gauge theory, the coupling constant of the D1-brane gauge theory $g_{1}^{2}=g_{s}\left(2 \pi \alpha^{\prime}\right)$ and the coupling constant of the D5-brane gauge theory $g_{5}^{2}=g_{s}\left(2 \pi \alpha^{\prime} \tilde{v}\right.$ Here $\tilde{v}$ is related to the volume of $T^{4}$ by $V_{T^{4}}=\alpha^{\prime 2}(2 \pi)^{4} \tilde{v}$. Since we are interested in low energies $E \ll 1 / l_{s}$ the gauge theory is strongly coupled.

7. In the above discussion from the geometry of the configuration, the fields $X_{i}^{(1,5)}$ along the torus directions and the fields $\chi$ are compact, since they parmetrize positions along the compact directions. However, it is not consistent with gauge invariance to take hypermultiplets of the $\mathcal{N}=2$ mulitplet to be compact ${ }^{7}$. Therefore the hypermultiplets are non-compact. Since we are interested in energies $E \ll 1 / l_{s}$ the expectation values of the hypers (which have units of energy ) $X_{i}^{(1,5)}, \chi \ll 1 / l_{s}$. Thus, the $(1,1),(5,5)(1,5)$ strings do not probe the entire domain of $T^{4}$. Therefore even though geometrically we are on $T^{4}$ it is consistent with the fact that the hypers are non-compact as we are intersted in $E \ll 1 / l_{s}$.

8. The above discussion of domain of validity of the gauge theory ties in nicely with the fact that the gauge theory is an approximate description while the instanton moduli space description is a more global description. The gauge theory is valid when the hypers get small expectation values. The hypermultiplet of the gauge theory corresponds to the scales of the instanton (via the ADHM construction for instantons on $R^{4}$ ). Thus the gauge theory is valid when the instantons have shrunk to almost zero size [110.

In summary, the gauge theory of the D1-D5 system is a $1+1$ dimensional $(4,4)$ supersymmetric gauge theory with gauge group $U\left(Q_{1}\right) \times U\left(Q_{5}\right)$. The matter content of this theory consists of hypermultiplets $Y^{(1)}$ 's, $Y^{(5)}$ 's transforming as adjoints of $U\left(Q_{1}\right)$ and $U\left(Q_{5}\right)$ respectively. It also has the hypermultiplets $\chi$ 's which transform as bi-fundamentals of $U\left(Q_{1}\right) \times \overline{U\left(Q_{5}\right)}$.

\subsection{The Potential Terms}

The Lagrangian of the above gauge theory can be worked out from the dimensional reduction of $d=6, \mathcal{N}=1$ gauge theory. The potential energy density of the vector and hyper multiplets is a sum of 4 positive terms (in this section for convenience

\footnotetext{
${ }^{7}$ This can be seen as follows. The D-term equations in the $\mathcal{N}=2$ theory admit a symmetry under the complexified gauge symmetry $G L\left(C, Q_{1}\right) \times G L\left(C, Q_{5}\right)$, (see for instance in [113]). This symmetry involves an arbitary scaling.
} 
of notation we have defined $Y_{i}^{(1)}=Y_{i}, Y_{i}^{(5)}=X_{i}, Y_{m}^{(1)}=Y_{m}, Y_{m}^{(5)}=X_{m}$ ) [48, 111] :

$$
\begin{aligned}
V= & V_{1}+V_{2}+V_{3}+V_{4} \\
V_{1}= & -\frac{1}{4 g_{1}^{2}} \sum_{m, n} \operatorname{Tr}_{U\left(Q_{1}\right)}\left[Y_{m}, Y_{n}\right]^{2}-\frac{1}{4 g_{5}^{2}} \sum_{m, n} \operatorname{Tr}_{U\left(Q_{5}\right)}\left[X_{m}, X_{n}\right]^{2} \\
V_{2}= & -\frac{1}{2 g_{1}^{2}} \sum_{i, m} \operatorname{Tr}_{U\left(Q_{1}\right)}\left[Y_{i}, Y_{m}\right]^{2}-\frac{1}{2 g_{5}^{2}} \sum_{i, m} \operatorname{Tr}_{U\left(Q_{5}\right)}\left[X_{i}, X_{m}\right]^{2} \\
V_{3}= & \frac{1}{4} \sum_{m} \operatorname{Tr}_{U\left(Q_{1}\right)}\left(\chi X_{m}-Y_{m} \chi\right)\left(X_{m} \chi^{\dagger}-\chi^{\dagger} Y_{m}\right) \\
V_{4}= & \frac{1}{4} \operatorname{Tr}_{U\left(Q_{1}\right)}\left(\chi i \Gamma_{i j}^{T} \chi^{+}+i\left[Y_{i}, Y_{j}\right]^{+}-\zeta_{i j}^{+} \frac{1}{Q_{1}}\right)^{2} \\
& +\frac{1}{4} \operatorname{Tr}_{U\left(Q_{5}\right)}\left(\chi^{+} i \Gamma_{i j} \chi+i\left[X_{i}, X_{j}\right]^{+}-\zeta_{i j}^{+} \frac{1}{Q_{5}}\right)^{2}
\end{aligned}
$$

The potential energy $V_{4}$ comes from a combination of $F$ and $D$ terms of the higher dimensional gauge theory. $\Gamma_{i j}=\frac{i}{2}\left[\Gamma_{i}, \Gamma_{j}\right]$ are spinor rotation matrices. The notation $a_{i j}^{+}$denotes the self-dual part of the anti-symmetric tensor $a_{i j}$.

In $V_{4}$ we have included the Fayet-Iliopoulos (FI) terms $\zeta_{i j}^{+}$, which form a triplet under $S U(2)_{R}$. Their inclusion is consistent with $\mathcal{N}=4$ SUSY. The FI terms can be identified with the self dual part of $B_{i j}$, the anti-symmetry tensor of the NS sector of the closed string theory [114. This identification at this stage rests on the fact that $(\mathrm{i}) \zeta_{i j}^{+}$and $B_{i j}^{+}$have identical transformation properties under $S U(4)_{I}$ and (ii) at the origin of the Higgs branch where $\chi=X=Y=0, V_{4} \sim \zeta_{i j}^{+} \zeta_{i j}^{+}$. This signals a tachyonic mode from the view point of string perturbation theory [114]. The tachyon mass is easily computed and this implies the relation $\zeta_{i j}^{+} \zeta_{i j}^{+} \sim B_{i j}^{+} B_{i j}^{+}$. These issues are discussed further in Section 7.

\subsection{D-Flatness Equations and the Moduli Space}

The supersymmetric ground state (semi-classical) is characterized by the 2-sets of D-flatness equations which are obtained by setting $V_{4}=0$. They are best written in terms of the $S U(2)_{R}$ doublet fields $N^{(1)}$ and $N^{(5)}$ :

$$
\begin{aligned}
& N^{(1)}=\left(\begin{array}{c}
N_{1}^{(1)} \\
N_{2}^{(1) \dagger}
\end{array}\right)=\left(\begin{array}{c}
Y_{9}+i Y_{8} \\
Y_{7}+i Y_{6}
\end{array}\right) \\
& N^{(5)}=\left(\begin{array}{c}
N_{1}^{(5)} \\
N_{2}^{(5) \dagger}
\end{array}\right)=\left(\begin{array}{l}
X_{9}+i X_{8} \\
X_{7}+i X_{9}
\end{array}\right)
\end{aligned}
$$

We also define $\zeta=\zeta_{69}^{+}$and $\zeta_{c}=\zeta_{67}^{+}+i \zeta_{68}^{+}$. With these definitions the 2 sets of D-flatness conditions become:

$$
\left(A A^{\dagger}-B^{\dagger} B\right)_{a^{\prime} b^{\prime}}+\left[N_{1}^{(1)}, N_{1}^{(1) \dagger}\right]_{a^{\prime} b^{\prime}}-\left[N_{2}^{(1)}, N_{2}^{(1) \dagger}\right]_{a^{\prime} b^{\prime}}=\frac{\zeta}{Q_{1}} \delta_{a^{\prime} b^{\prime}}
$$




$$
\begin{aligned}
(A B)_{a^{\prime} b^{\prime}}+\left[N_{1}^{(1)}, N_{2}^{(1) \dagger}\right]_{a^{\prime} b^{\prime}} & =\frac{\zeta_{c}}{Q_{1}} \delta_{a^{\prime} b^{\prime}} \\
\left(A^{\dagger} A-B B^{\dagger}\right)_{a b}+\left[N_{1}^{(5)}, N_{1}^{(5) \dagger}\right]_{a b}-\left[N_{2}^{(5)}, N_{2}^{(5) \dagger}\right]_{a b} & =\frac{\zeta}{Q_{5}} \delta_{a b} \\
\left(A^{+} B^{+}\right)_{a b}+\left[N_{1}^{(5)}, N_{2}^{(5) \dagger}\right]_{a b} & =\frac{\zeta_{c}}{Q_{5}} \delta_{a b}
\end{aligned}
$$

Here $a^{\prime}, b^{\prime}$ runs from $1 \cdots Q_{1}$ and $a, b$ runs from $1 \cdots Q_{5}$. The hypermultiplet moduli space is a solution of the above equations modulo the gauge group $U\left(Q_{1}\right) \times U\left(Q_{5}\right)$. A detailed discussion of the procedure was given in [111, 88]. Below we summarize the main points.

If we take the trace parts of (4.13) we get the same set of 3 equations as the Dflatness equations for a $U(1)$ theory with $Q_{1} Q_{5}$ hypermultiplets, with $U(1)$ charge assignment $(+1,-1)$ for $\left(A_{a^{\prime} b}, B_{a^{\prime} b}^{T}\right)$. Thus,

$$
\begin{aligned}
\sum_{a^{\prime} b}\left(A_{a^{\prime} b} A_{a^{\prime} b}^{*}-B_{a^{\prime} b}^{T} B_{a^{\prime} b}^{T *}\right) & =\zeta \\
\sum_{a^{\prime} b} A_{a^{\prime} b} B_{a^{\prime} b}^{T} & =\zeta_{c}
\end{aligned}
$$

For a given point on the surface defined by $(4.14), 4.15$ the traceless parts of (4.13) lead to $3 Q_{1}^{2}+3 Q_{5}^{2}-6$ constraints among $4 Q_{1}^{2}+4 Q_{5}^{2}-8$ degrees of freedom corresponding to the traceless parts of the adjoint hypermultiplets $N^{(1)}$ and $N^{(5)}$. Using $Q_{1}^{2}+Q_{5}^{2}-2$ gauge conditions corresponding to $S U\left(Q_{1}\right) \times S U\left(Q_{5}\right)$ we have $\left(3 Q_{1}^{2}+3 Q_{5}^{2}-6\right)+\left(Q_{1}^{2}+Q_{5}^{2}-2\right)=4 Q_{1}^{2}+4 Q_{5}^{2}-8$ conditions for the $\left(4 Q_{1}^{2}+4 Q_{5}^{2}-8\right)$ degrees of freedom in the traceless parts of $N^{(1)}$ and $N^{(5)}$. The 8 degrees of freedom corresponding to $\operatorname{Tr} X_{i}$ and $\operatorname{Tr} Y_{i}, i=6,7,8,9$ correspond to the centre-of-mass of the D5 and D1 branes respectively.

\subsection{The Bound State in the Higgs Phase}

Having discussed the moduli space that characterizes the SUSY ground state we can discuss the fluctuations of the transverse vector multiplet scalars $X_{m}$ and $Y_{m}$, $m=1,2,3,4$. In the Higgs phase since $\left\langle X_{m}\right\rangle=\left\langle Y_{m}\right\rangle=0$ and $\chi=\bar{\chi}$ lies on the surface defined by (4.14), 4.15). The relevant action of fluctuations in the path integral is,

$$
\begin{aligned}
S= & \sum_{m} \int d t d x_{5}\left(\operatorname{Tr}_{U\left(Q_{5}\right)} \partial_{\alpha} X_{m} \partial^{\alpha} X_{m}+\operatorname{Tr}_{U\left(Q_{1}\right)} \partial_{\alpha} Y_{m} \partial^{\alpha} Y_{m}\right) \\
& +\int d t d x_{5}\left(V_{2}+V_{3}\right)
\end{aligned}
$$

We restrict the discussion to the case when $Q_{5}=1$ and $Q_{1}$ is arbitrary. In this case the matrix $X_{m}$ is a real number which we denote by $x_{m} \cdot \chi$ is a complex column vector with components $\left(A_{a^{\prime}}, B_{a^{\prime}}\right)$. Since we are looking at the fluctuations of the 
$Y_{m}$ only to quadratic order in the path integral, the integrals over the different $Y_{m}$ decouple from each other and we can treat each of them separately. Let us discuss the fluctuation $Y_{1}$ and set $\left(Y_{1}\right)_{a^{\prime} b^{\prime}}=\delta_{a^{\prime} b^{\prime}} y_{1 a^{\prime}}$. Then the potential $V_{3}$, (4.10) becomes

$$
V_{3}=\sum_{a^{\prime}}\left(\left|A_{a^{\prime}}\right|^{2}+\left|B_{a^{\prime}}\right|^{2}\right)\left(y_{1 a^{\prime}}-x_{1}\right)^{2}
$$

We will prove that $\left|A_{a^{\prime}}\right|^{2}+\left|B_{a^{\prime}}\right|^{2}$ can never vanish if the FI terms are non-zero. In order to do this let us analyze the second D-term equation (4.13)

$$
A_{a^{\prime}} B_{b^{\prime}}+\left[N_{1}^{(1)}, N_{2}^{(1) \dagger}\right]_{a^{\prime} b^{\prime}}=\frac{\zeta_{c}}{Q_{1}} \delta_{a^{\prime} b^{\prime}}
$$

We can use the complex gauge group $G L\left(C, Q_{1}\right)$ to diagonalize the complex matrix $N_{1}^{(1)}$ [113. Then, (4.18) becomes

$$
A_{a^{\prime}} B_{b^{\prime}}+\left(n_{a^{\prime}}-n_{b^{\prime}}\right)\left(N_{2}^{(1) \dagger}\right)_{a^{\prime} b^{\prime}}=\frac{\zeta_{c}}{Q_{1}} \delta_{a^{\prime} b^{\prime}}
$$

For $a^{\prime} \neq b^{\prime}$, this determines the non-diagonal components of $N_{2}^{(1)}$

$$
\left(N_{2}^{(1) \dagger}\right)_{a^{\prime} b^{\prime}}=-\frac{A_{a^{\prime}} B_{b^{\prime}}}{n_{a^{\prime}}-n_{b^{\prime}}}
$$

For $a=b$, we get the equations

$$
A_{a^{\prime}} B_{a^{\prime}}=\frac{\zeta_{c}}{Q_{1}}
$$

which imply that

$$
\left|A_{a^{\prime}}\right|\left|B_{a^{\prime}}\right|=\frac{\left|\zeta_{c}\right|}{Q_{1}}
$$

with the consequence that $\left|A_{a^{\prime}}\right|$ and $\left|B_{a^{\prime}}\right|$ are non-zero for all $a^{\prime}=1, . ., Q_{1}$. This implies that $\left.\left(\left|A_{a^{\prime}}\right|^{2}+\left|B_{a^{\prime}}\right|^{2}\right)>0\right)$, and hence the fluctuation $\left(y_{1 a^{\prime}}-x_{1}\right)$ is massive. If we change variables $y_{1 a^{\prime}} \rightarrow y_{1 a^{\prime}}+x_{1}$, then $x_{1}$ is the only flat direction. This corresponds to the global translation of the 5 -brane in the $x_{1}$ direction.

A similar analysis can be done for all the remaining directions $m=2,3,4$ with identical conclusions. This shows that a non-zero FI term implies a true bound state of the $Q_{5}=1, Q_{1}=N$ system. If $F I=0$, then there is no such guarantee and the system can easily fragment, due to the presence of flat directions in $\left(Y_{m}\right)_{a^{\prime} b^{\prime}}$.

What the above result says is that when the FI parameters are non-zero the zero mode of the fields $\left(Y_{m}\right)_{a^{\prime} b^{\prime}}$ is massive. If we regard the zero mode as a collective coordinate then the Hamiltonian of the zero mode has a quadratic potential which agrees with the near horizon limit of the Liouville potential derived in [114, 88].

The general case with an arbitrary number of $Q_{1}$ and $Q_{5}$ branes seems significantly harder to prove and is an open question, but the result is very plausible on physical grounds. If the potential for a single test $D 1$ brane is attractive, it is hard to imagine any change in this fact if there are 2 test $D 1$ branes, because the $D 1$ branes by themselves can form a bound state. 


\subsection{The Conformally Invariant Limit of the Gauge Theory}

In the previous section we showed that the D1-D5 system leads to a bound state in the Higgs phase. The next question is about the low energy collective excitations of the bound state. They are described by the sigma model corresponding to the hyper-multiplet moduli defined by the equations (4.13). The Lagrangian (bosonic part) is

$$
\begin{aligned}
S= & \sum_{m} \int d t d x_{5}\left(\operatorname{tr}_{U\left(Q_{5}\right)} \partial_{\alpha} X_{i} \partial^{\alpha} X_{i}+\operatorname{tr}_{U\left(Q_{1}\right)} \partial_{\alpha} Y_{i} \partial^{\alpha} Y_{i}\right) \\
& +\int d t d x_{5}\left(\partial_{\alpha} \chi \partial^{\alpha} \chi^{\dagger}\right)
\end{aligned}
$$

This is a difficult non-linear system, with $\mathcal{N}=4$ SUSY. Since we are interested in the low energy dynamics we may as well ask whether there is a SCFT fixed point. This SCFT fixed point is relevant in the study of the near horizon geometry (2.71). Such a SCFT must have $(4,4)$ supersymmetry (16 real supersymmetries) with a central charge $c=6\left(Q_{1} Q_{5}+1\right)$. Now note that the equations (4.14), (1.15) describe a hyper-Kahler manifold and hence the sigma model defined on it is a SCFT with $(4,4)$ SUSY. We can then consider the part of the action involving the $X_{i}$ and $Y_{i}$ which are solved in terms of the $\chi$ as giving a deformation of the SCFT. Now this deformation clearly breaks the superconformal symmetry.

The sigma model action at the conformally invariant point is

$$
\int d t d x_{5} \sum_{a^{\prime} b}\left(\partial_{\alpha} A_{a^{\prime} b} \partial_{\alpha} A_{a^{\prime} b}^{*}+\partial_{\alpha} B_{a^{\prime} b}^{T} \partial_{\alpha} B_{a^{\prime} b}^{T *}\right)
$$

The sigma model fields are constrained to be on the surface defined by (4.14), (4.15). Further after appropriate gauge fixing the residual gauge invariance inherited from the gauge theory is the Weyl group $S\left(Q_{1}\right) \times S\left(Q_{5}\right)$ [11]. The Weyl invariance can be used to construct gauge invariant strings of various lengths. If $Q_{1}$ and $Q_{5}$ are relatively prime it is indeed possible to prove the existence of a single winding string with minimum unit of momentum given by $\frac{1}{Q_{1} Q_{5}}$. This is associated with the longest cyclic subgroup of $S\left(Q_{1}\right) \times S\left(Q_{5}\right)$. Cyclic subgroups of shorter length cycles

lead to strings with minimum momentum $\frac{1}{l_{1} l_{5}}$, where $l_{1}$ and $l_{5}$ are the lengths of the cycles [111]. In a different way of describing these degrees of freedom we shall see in the next sections that strings of various lengths are associated with chiral primary operators of the conformal field theory on the moduli space of instantons on a 4 -torus.

\subsection{Quick derivation of entropy and temperatures from CFT}

We pause in this section to show that certain deductions about thermodynamic properties can be made just by the knowledge of the central charge $\left(c=6 Q_{1} Q_{5}\right)$ and the level of the Virasoro algebra of the unitary superconformal field theory 
mentioned above. This information is sufficient to calculate the number of microstates (a more detailed and complete derivation is given in Section 8).

To find the microstates of the D1-D5 black hole we look for states with $L_{0}=N_{L}$ and $\bar{L}_{0}=N_{R}$. The asymptotic number of distinct states of this SCFT is given by Cardy's formula [115]

$$
\begin{aligned}
\Omega & =\Omega_{L} \Omega_{R} \\
\Omega_{L, R} & =\exp \left[2 \pi \sqrt{c N_{L, R} / 6}\right]=\exp \left[2 \pi \sqrt{Q_{1} Q_{5} N_{L, R}}\right]
\end{aligned}
$$

From the Boltzmann formula one obtains

$$
S=\ln \Omega=2 \pi\left(\sqrt{Q_{1} Q_{5} N_{L}}+\sqrt{Q_{1} Q_{5} N_{R}}\right)
$$

This exactly reproduces the Bekenstein-Hawking entropy (2.47). The exact agreement is surprising since for arbitrary $N_{R}, N_{L} \neq 0$ the states being considered are far from being supersymmetric.

The quantity $\Omega_{L}\left(N_{L}\right)$ defines (the number of states in) a microcanonical ensemble, in which we fix the energy of the left-movers to be

$$
E_{L}=\frac{N_{L}}{R_{5}}
$$

The equivalent canonical ensemble is defined by

$$
\begin{aligned}
Z_{L} & \equiv \operatorname{Tr} \exp \left[-\beta_{L} E_{L}\right]=\sum_{N_{L}=0}^{\infty} \Omega_{L}\left(N_{L}\right) \exp \left[-\beta_{L} N_{L} / R_{5}\right] \\
& =\sum_{N_{L}=0}^{\infty} \exp \left[2 \pi \sqrt{Q_{1} Q_{5} N_{L}}-\beta_{L} N_{L} / R_{5}\right]
\end{aligned}
$$

For large enough temperature $T_{L}\left(=1 / \beta_{L}\right)$, the sum in the second line is dominated by a saddle point value that occurs at

$$
\frac{\beta_{L}}{R_{5}}=\pi \sqrt{\frac{Q_{1} Q_{5}}{N_{L}}}
$$

This determines the temperature of the left-movers; a similar reasoning works for the right movers. The two temperatures are given by

$$
T_{L}=\frac{1}{\beta_{L}}=\frac{1}{\pi R_{5}} \sqrt{\frac{N_{L}}{Q_{1} Q_{5}}} \text { and } T_{R}=\frac{1}{\beta_{R}}=\frac{1}{\pi R_{5}} \sqrt{\frac{N_{R}}{Q_{1} Q_{5}}}
$$

The temperature $T_{H}=1 / \beta_{H}$ of the full system is conjugate to the total energy $E=E_{L}+E_{R}$, and is given by $^{8}$

$$
\begin{aligned}
& \beta_{H}=\frac{1}{2}\left(\beta_{L}+\beta_{R}\right) \Rightarrow \frac{1}{T_{H}}=\frac{1}{2}\left(\frac{1}{T_{L}}+\frac{1}{T_{R}}\right) \\
& T_{H}=1 / \beta_{H}=\frac{2 \sqrt{N_{L} N_{R}}}{\pi R_{5} \sqrt{Q_{1} Q_{5}}\left(\sqrt{N_{L}}+\sqrt{N_{R}}\right)}
\end{aligned}
$$

\footnotetext{
${ }^{8}$ To derive the "harmonic" rule in (4.30) (cf. also (3.23)), define the full partition function as $Z \equiv Z_{L} Z_{R}=\operatorname{Tr} \exp \left[-\left(\beta_{L} E_{L}+\beta_{R} E_{R}\right)\right]$
} 


\section{Comparison with Supergravity}

We have so far encountered three expressions for temperature, in Eq. 2.45) in Section 2, in Eqs. (3.16),(3.23) in Section 3, and the expressions (4.29),(4.31) above. It will be interesting to compare them.

Note that the dilute gas regime (3.9) implies (see Eqs (2.40))

$$
\sinh \alpha_{1}, \sinh \alpha_{5} \gg 1, \sinh \alpha_{n}
$$

In this region (see (2.41), 2.42$)$ )

$$
Q_{1,5} \approx N_{1,5} \gg N_{\overline{1}, \overline{5}}
$$

This gives

$$
r_{0} \cosh \alpha_{1,5} \approx \frac{r_{0}}{2} \exp \left[\alpha_{1,5}\right] \approx \sqrt{c_{1,5} Q_{1,5}}
$$

Eq. (2.42) gives

$$
r_{0} e_{n}^{\alpha}=2 \sqrt{c_{n} N_{L}}, r_{0} e^{-\alpha_{n}}=2 \sqrt{c_{n} N_{R}}
$$

Using these expressions (and also (2.34)) it is easy to see that (2.45), (3.23) both reduce to (4.31). Also (3.16) reduce to (4.29).

\subsection{D1 branes as solitonic strings of the D5 gauge theory}

In the previous subsection we found that the Higgs branch of the gauge theory of the D1-D5 system flows in the infrared to $\mathcal{N}=(4,4)$ SCFT on a target space $\widetilde{\mathcal{M}}$ with central charge $6 Q_{1} Q_{5}$ (we have excluded the centre of mass degrees of freedom). For black hole processes like Hawking radiation it is important to have a better handle on the target space $\mathcal{M}$. In this section we review the arguments which show that the target space $\widetilde{\mathcal{M}}$ is a resolution of the orbifold $T^{4} \times\left(\tilde{T}^{4}\right)^{Q_{1} Q_{5}} / S\left(Q_{1} Q_{5}\right)$. (We use the notation $\tilde{T}^{4}$ to distinguish it from the compactification torus $T^{4}$.) This discussion gives a succinct description of the bound state in the Higgs phase.

which we can rewrite as

$$
Z=\operatorname{Tr} \exp \left[-\frac{1}{2}\left(\beta_{L}+\beta_{R}\right)\left(E_{L}+E_{R}\right)-\frac{1}{2}\left(\beta_{L}-\beta_{R}\right)\left(E_{L}-E_{R}\right)\right]
$$

Performing the sum over $E_{L}-E_{R}$ (equivalently, over $N_{L}-N_{R}$ ), and ignoring the unimportant multiplicative constant we get

$$
Z=\operatorname{Tr} \exp \left[-\beta_{H}\left(E_{L}+E_{R}\right)\right]
$$

where

$$
\beta_{H}=\frac{1}{2}\left(\beta_{L}+\beta_{R}\right)
$$


The $Q_{1}$ D1-branes can be thought of as $Q_{1}$ instantons in the $5+1$ dimensional $U\left(Q_{5}\right)$ super Yang-Mills theory of the $Q_{5}$ D5-branes [116, 117]. To see this note that the DBI action of the D5-branes have a coupling

$$
\int d^{6} x C^{(2)} \wedge \operatorname{Tr}\left[F^{(5)} \wedge F^{(5)}\right]
$$

The non-trivial gauge configurations which are independent of $x^{0}, x^{5}$ and have zero values of $A_{0}^{(5)}$ and $A_{5}^{(5)}$ but non-zero values of $\operatorname{Tr}\left[F^{(5)} \wedge F^{(5)}\right]$ act as sources of the Ramond-Ramond two-form $C_{05}^{(2)}$. If these gauge field configurations have to preserve half the supersymmetries of the D5-brane action they should be self dual. Thus they are instanton solutions of four-dimensional Euclidean Yang-Mills of the $6,7,8,9$ directions.

Additional evidence for this comes from the fact that the integral property of $\operatorname{Tr}\left[F^{(5)} \wedge F^{(5)}\right]$ corresponds to the quantization of the D1-branes charge. The action for a $Q_{1}$ instanton solution is $Q_{1} / g_{Y M}^{2}$. This agrees with the tension of $Q_{1}$ D1-branes, namely $Q_{1} / g_{s}$. If one is dealing with non-compact D5-branes and D1branes it is seen that the D-flatness conditions of the D1-brane theory is identical to the ADHM construction of $Q_{1}$ instantons of $U\left(Q_{5}\right)$ gauge theory [117. In fact, the last two equations in (4.13) are the relevant ADHM equations in this case [118].

From the discussion in the preceding paragraphs we conclude that, excluding Wilson lines of the $U\left(Q_{5}\right)$ gauge theory, $\widetilde{\mathcal{M}}$ can be thought of as the moduli space of $Q_{1}$ instantons of a $U\left(Q_{5}\right)$ gauge theory on $T^{4}$. This moduli space is known to be the Hilbert scheme of $Q_{1} Q_{5}$ points on $\tilde{T}^{4}$ [119. $\tilde{T}^{4}$ can be different from the compactification torus $T^{4}$. This is a smooth resolution of the singular orbifold $\left(\tilde{T}^{4}\right)^{Q_{1} Q_{5}} / S\left(Q_{1} Q_{5}\right)$. We will provide physically motivated evidence for the fact that the moduli space of $Q_{1}$ instantons of a $U\left(Q_{5}\right)$ gauge theory on $T^{4}$ is a smooth resolution of the orbifold $\left(\tilde{T}^{4}\right)^{Q_{1} Q_{5}} / S\left(Q_{1} Q_{5}\right)$ using string dualities. The evidence is topological and it comes from realizing that the cohomology of $\widetilde{\mathcal{M}}$ is the degeneracy of the ground states of the D1-D5 gauge theory. We can calculate this degeneracy in two ways. One is by explicitly counting the cohomology of $\left(\tilde{T}^{4}\right)^{Q_{1} Q_{5}} / S\left(Q_{1} Q_{5}\right)$ [120]. The second method is to use string dualities as discussed below. Both these methods give identical answers. Thus at least at the level of cohomology we are able to verify that the moduli space of $Q_{1}$ instantons of a $U\left(Q_{5}\right)$ gauge theory on $T^{4}$ is smooth resolution of $\left(\tilde{T}^{4}\right)^{Q_{1} Q_{5}} / S\left(Q_{1} Q_{5}\right)$.

Consider type IIB string theory compactified on $S^{1} \times T^{4}$ with a fundamental string having $Q_{5}$ units of winding along $x^{6}$ and $Q_{1}$ units of momentum along $x^{6}$. On performing the sequence of dualities $S T_{6789} S T_{56}$ we can map the fundamental string to the D1-D5 system (we can de-compactify the $x^{5}$ direction finally) with $Q_{1}$ D1-branes along $x^{5}$ and $Q_{5}$ D5-branes along $x^{5}, x^{6}, x^{7}, x^{8}, x^{9}$ [121]. Therefore using this U-duality sequence the BPS states of this fundamental string (that is, states with either purely left moving or right moving oscillators) maps to ground states of 
the D1-D5 system. The number of ground states of the D1-D5 system is given by the dimension of the cohomology of $\widetilde{\mathcal{M}}$. From the perturbative string degeneracy counting the generating function of BPS states with left moving oscillator number $N_{L}$ is given by

$$
\sum_{N_{L}=o}^{\infty} d\left(N_{L}\right) q^{N_{L}}=256 \times \prod_{n=1}^{\infty}\left(\frac{1+q^{n}}{1-q^{n}}\right)^{8}
$$

where $d\left(N_{L}\right)$ refers to the degeneracy of states with left moving oscillator number $N_{L}$. The D1-D5 system is U-dual to the perturbative string with $N_{L}=Q_{1} Q_{5}$.

Explicit counting of the cohomology of $\left(\tilde{T}^{4}\right)^{Q_{1} Q_{5}} / S\left(Q_{1} Q_{5}\right)$ gives $d\left(Q_{1} Q_{5}\right) / 256$. The factor 256 comes from quantization of the center of mass coordinate along $1,2,3,4$ directions and the $6,7,8,9$ directions. The center of mass coordinate is represented by the $U(1)$ of $U\left(Q_{1}\right) \times U\left(Q_{5}\right)$. Therefore the low energy theory of the bound D1-D5 system is a SCFT on the target space

$$
R^{4} \times T^{4} \times\left(\tilde{T}^{4}\right)^{Q_{1} Q_{5}} / S\left(Q_{1} Q_{5}\right)
$$

It is also useful to interpret the moduli represented by the free $T^{4}$ form the gauge theory of $Q_{5}$ D5-branes. As this theory is on a torus $T^{4}$ it admits Wilson-lines along all the cyles of the $T^{4}$. The free torus in the above equation stand for Wilson-lines in the theory of the D5-branes. From the D1-D5 gauge theory point of view the free $T^{4}$ belongs to the Higgs branch as it stands for the center of mass coordinate on $T^{4}$ parametrized by $\operatorname{Tr}_{\mathrm{U}\left(\mathrm{Q}_{5}\right)}\left(\mathrm{Y}_{\mathrm{i}}^{(5)}\right)+\operatorname{Tr}_{\mathrm{U}\left(\mathrm{Q}_{1}\right)}\left(\mathrm{Y}_{\mathrm{i}}^{(1)}\right)$ while $R^{4}$ belongs to the Coulomb branch. It is parameterized by $\operatorname{Tr}_{\mathrm{U}\left(\mathrm{Q}_{5}\right)}\left(\mathrm{Y}_{\mathrm{m}}^{(5)}\right)+\operatorname{Tr}_{\mathrm{U}\left(\mathrm{Q}_{1}\right)}\left(\mathrm{Y}_{\mathrm{m}}^{(1)}\right)$. Thus the Higgs branch of the D1-D5 gauge theory flows in the infrared to a $\mathcal{N}=(4,4)$ SCFT on $T^{4} \times\left(\tilde{T}^{4}\right)^{Q_{1} Q_{5}} / S\left(Q_{1} Q_{5}\right)$. The SCFT on $T^{4}$ is free while the symmetric product contains interesting dynamics. From now on we will denote the symmetric product orbifold $\left(\tilde{T}^{4}\right)^{Q_{1} Q_{5}} / S\left(Q_{1} Q_{5}\right)$ by $\mathcal{M}$. 


\section{The SCFT on the orbifold $\mathcal{M}$}

As we have seen in the last section the Higgs branch of the D1-D5 system flows in the infrared to a product of a $\mathcal{N}=(4,4) \mathrm{SCFT}$ on a resolution of the orbifold $\mathcal{M}$ with a free theory on $T^{4}$. As the SCFT on $T^{4}$ is free and decoupled we will first focus on the symmetric product. We will formulate the SCFT on $\mathcal{M}$ as a free field theory with identifications and discuss its symmetries. In particular we find a new $S O(4)$ algebra which is useful in the classification of states. We then construct the operators which correspond to the moduli of the SCFT including the four operators which correspond to the resolution of the orbifold. Finally we explicitly construct the chiral primaries of the $\mathcal{N}=(4,4)$ SCFT on the symmetric product orbifold $\mathcal{M}$.

The $\mathcal{N}=(4,4)$ SCFT on $\mathcal{M}$ is described by the free Lagrangian

$$
S=\frac{1}{2} \int d^{2} z\left[\partial x_{A}^{i} \bar{\partial} x_{i, A}+\psi_{A}^{i}(z) \bar{\partial} \psi_{A}^{i}(z)+\widetilde{\psi}_{A}^{i}(\bar{z}) \partial \widetilde{\psi}_{A}^{i}(\bar{z})\right]
$$

Here $i$ runs over the $\widetilde{T^{4}}$ coordinates $1,2,3,4$ and $A=1,2, \ldots, Q_{1} Q_{5}$ labels various copies of the four-torus. The symmetric group $S\left(Q_{1} Q_{5}\right)$ acts by permuting the copy indices. It introduces various twisted sectors which we will discuss later. The free field realization of this $\mathrm{SCFT}$ has $\mathcal{N}=(4,4)$ superconformal symmetry. To set up our notations and conventions we review the $\mathcal{N}=4$ superconformal algebra.

\subsection{The $\mathcal{N}=4$ superconformal algebra}

The algebra is generated by the stress energy tensor, four supersymmetry currents, and a local $S U(2) R$ symmetry current. The operator product expansions(OPE) of the algebra with central charge $c$ are given by (See for example [122].)

$$
\begin{aligned}
T(z) T(w) & =\frac{\partial T(w)}{z-w}+\frac{2 T(w)}{(z-w)^{2}}+\frac{c}{2(z-w)^{4}}, \\
G^{a}(z) G^{b \dagger}(w) & =\frac{2 T(w) \delta_{a b}}{z-w}+\frac{2 \bar{\sigma}_{a b}^{i} \partial J^{i}}{z-w}+\frac{4 \bar{\sigma}_{a b}^{i} J^{i}}{(z-w)^{2}}+\frac{2 c \delta_{a b}}{3(z-w)^{3}}, \\
J^{i}(z) J^{j}(w) & =\frac{i \epsilon^{i j k} J^{k}}{z-w}+\frac{c}{12(z-w)^{2}}, \\
T(z) G^{a}(w) & =\frac{\partial G^{a}(w)}{z-w}+\frac{3 G^{a}(z)}{2(z-w)^{2}} \\
T(z) G^{a \dagger}(w) & =\frac{\partial G^{a \dagger}(w)}{z-w}+\frac{3 G^{a \dagger}(z)}{2(z-w)^{2}} \\
T(z) J^{i}(w) & =\frac{\partial J^{i}(w)}{z-w}+\frac{J^{i}}{(z-w)^{2}}, \\
J^{i}(z) G^{a}(w) & =\frac{G^{b}(z)\left(\sigma^{i}\right)^{b a}}{2(z-w)}
\end{aligned}
$$




$$
J^{i}(z) G^{a \dagger}(w)=-\frac{\left(\sigma^{i}\right)^{a b} G^{b \dagger}(w)}{2(z-w)}
$$

Here $T(z)$ is the stress energy tensor, $G^{a}(z), G^{b \dagger}(z)$ the $S U(2)$ doublet of supersymmetry generators and $J^{i}(z)$ the $S U(2) R$ symmetry current. The $\sigma$ 's stand for Pauli matrices and the $\bar{\sigma}$ 's stand for the complex conjugates of Pauli matrices. In the free field realization described below, the above holomorphic currents occur together with their antiholomorphic counterparts, which we will denote by $\widetilde{J}(\bar{z}), \widetilde{G}(\bar{z})$ and $\widetilde{T}(\bar{z})$. In particular, the R-parity group will be denoted by $S U(2)_{R} \times S \widetilde{S U}(2)_{R}$.

\subsection{Free field realization of $\mathcal{N}=(4,4)$ SCFT on the orbifold $\mathcal{M}$}

A free field realization of the $\mathcal{N}=4$ superconformal algebra with $c=6 Q_{1} Q_{5}$ can be constructed out of $Q_{1} Q_{5}$ copies of four real fermions and bosons. The generators are given by

$$
\begin{aligned}
T(z) & =\partial X_{A}(z) \partial X_{A}^{\dagger}(z)+\frac{1}{2} \Psi_{A}(z) \partial \Psi_{A}^{\dagger}(z)-\frac{1}{2} \partial \Psi_{A}(z) \Psi_{A}^{\dagger}(z) \\
G^{a}(z) & =\left(\begin{array}{c}
G^{1}(z) \\
G^{2}(z)
\end{array}\right)=\sqrt{2}\left(\begin{array}{c}
\Psi_{A}^{1}(z) \\
\Psi_{A}^{2}(z)
\end{array}\right) \partial X_{A}^{2}(z)+\sqrt{2}\left(\begin{array}{c}
-\Psi_{A}^{2 \dagger}(z) \\
\Psi_{A}^{1 \dagger}(z)
\end{array}\right) \partial X_{A}^{1}(z) \\
J_{R}^{i}(z) & =\frac{1}{2} \Psi_{A}(z) \sigma^{i} \Psi_{A}^{\dagger}(z)
\end{aligned}
$$

We will use the following notation for the zero mode of the R-parity current:

$$
J_{R}^{i}=\frac{1}{2} \int \frac{d z}{2 \pi i} \Psi_{A}(z) \sigma^{i} \Psi_{A}^{\dagger}(z)
$$

In the above the summation over $A$ which runs from 1 to $Q_{1} Q_{5}$ is implied. The bosons $X$ and the fermions $\Psi$ are

$$
\begin{aligned}
& X_{A}(z)=\left(X_{A}^{1}(z), X_{A}^{2}(z)\right)=\sqrt{1 / 2}\left(x_{A}^{1}(z)+i x_{A}^{2}(z), x_{A}^{3}(z)+i x_{A}^{4}(z)\right), \\
& \Psi_{A}(z)=\left(\Psi_{A}^{1}(z), \Psi_{A}^{2}(z)\right)=\sqrt{1 / 2}\left(\psi_{A}^{1}(z)+i \psi_{A}^{2}(z), \psi_{A}^{3}(z)+i \psi_{A}^{4}(z)\right) \\
& X_{A}^{\dagger}(z)=\left(\begin{array}{c}
X_{A}^{1 \dagger}(z) \\
X_{A}^{2 \dagger}(z)
\end{array}\right)=\sqrt{\frac{1}{2}}\left(\begin{array}{c}
x_{A}^{1}(z)-i x_{A}^{2}(z) \\
x_{A}^{3}(z)-i x_{A}^{4}(z)
\end{array}\right) \\
& \Psi_{A}^{\dagger}(z)=\left(\begin{array}{c}
\Psi_{A}^{1 \dagger}(z) \\
\Psi^{2}{ }^{\dagger}(z)
\end{array}\right)=\sqrt{\frac{1}{2}}\left(\begin{array}{c}
\psi_{A}^{1}(z)-i \psi_{A}^{2}(z) \\
\psi_{A}^{3}(z)-i \psi_{A}^{4}(z)
\end{array}\right)
\end{aligned}
$$

\subsection{The $S O(4)$ algebra}

In addition to the local $R$ symmetry the free field realization of the $\mathcal{N}=4$ super-

conformal algebra has additional global symmetries which can be used to classify 
the states. There are 2 global $S U(2)$ symmetries which correspond to the $S O(4)$ rotations of the 4 bosons $x^{i}$. The corresponding charges are given by

$$
\begin{aligned}
I_{1}^{i} & =\frac{1}{4} \int \frac{d z}{2 \pi i} X_{A} \sigma^{i} \partial X_{A}^{\dagger}-\frac{1}{4} \int \frac{d z}{2 \pi i} \partial X_{A} \sigma^{i} X_{A}^{\dagger}+\frac{1}{2} \int \frac{d z}{2 \pi i} \Phi_{A} \sigma^{i} \Phi_{A}^{\dagger} \\
I_{2}^{i} & =\frac{1}{4} \int \frac{d z}{2 \pi i} \mathcal{X}_{A} \sigma^{i} \partial \mathcal{X}_{A}^{\dagger}-\frac{1}{4} \int \frac{d z}{2 \pi i} \partial \mathcal{X}_{A} \sigma^{i} \mathcal{X}_{A}^{\dagger}
\end{aligned}
$$

Here

$$
\begin{gathered}
\mathcal{X}_{A}=\left(X_{A}^{1},-X_{A}^{2 \dagger}\right) \quad \mathcal{X}^{\dagger}=\left(\begin{array}{c}
X_{A}^{1 \dagger} \\
-X_{A}^{2}
\end{array}\right) \\
\Phi_{A}=\left(\Psi_{A}^{1}, \Psi_{A}^{2 \dagger}\right) \quad \Phi_{A}^{\dagger}=\left(\begin{array}{c}
\Psi_{A}^{1 \dagger} \\
\Psi_{A}^{2}
\end{array}\right) .
\end{gathered}
$$

These charges are generators of $S U(2) \times S U(2)$ algebra:

$$
\begin{gathered}
{\left[I_{1}^{i}, I_{1}^{j}\right]=i \epsilon^{i j k} I_{1}^{k} \quad\left[I_{2}^{i}, I_{2}^{j}\right]=i \epsilon^{i j k} I_{2}^{k}} \\
{\left[I_{1}^{i}, I_{2}^{j}\right]=0}
\end{gathered}
$$

The commutation relation of these new global charges with the various local charges are given below

$$
\begin{aligned}
& {\left[I_{1}^{i}, G^{a}(z)\right]=0 \quad\left[I_{1}^{i}, G^{a \dagger}(z)\right]=0} \\
& {\left[I_{1}^{i}, T(z)\right]=0 \quad\left[I_{1}^{i}, J(z)\right]=0} \\
& {\left[I_{2}^{i}, \mathcal{G}^{a}(z)\right]=\frac{1}{2} \mathcal{G}^{b}(z) \sigma_{b a}^{i} \quad\left[I_{2}^{i}, \mathcal{G}^{a \dagger}(z)\right]=-\frac{1}{2} \sigma_{a b}^{i} \mathcal{G}^{b \dagger}(z)} \\
& {\left[I_{2}^{i}, T(z)\right]=0 \quad\left[I_{2}^{i}, J(z)\right]=0}
\end{aligned}
$$

where

$$
\mathcal{G}=\left(G^{1}, G^{2 \dagger}\right) \quad \mathcal{G}^{\dagger}=\left(\begin{array}{c}
G^{1 \dagger} \\
G^{2}
\end{array}\right)
$$

The following commutations relation show that the bosons transform as $(\mathbf{2}, \mathbf{2})$ under $S U(2)_{I_{1}} \times S U(2)_{I_{2}}$

$$
\begin{aligned}
{\left[I_{1}^{i}, X_{A}^{a}\right] } & =\frac{1}{2} X_{A}^{b} \sigma_{b a}^{i} & {\left[I_{1}^{i}, X_{A}^{a \dagger}\right] } & =-\frac{1}{2} \sigma_{a b}^{i} X_{A}^{b \dagger} \\
{\left[I_{2}^{i}, \mathcal{X}_{A}^{a}\right] } & =\frac{1}{2} \mathcal{X}_{A}^{b} \sigma_{b a}^{i} & {\left[I_{2}^{i}, \mathcal{X}_{A}^{a \dagger}\right] } & =-\frac{1}{2} \sigma_{a b}^{i} \mathcal{X}_{A}^{b \dagger}
\end{aligned}
$$

The fermions transform as $(\mathbf{2}, \mathbf{1})$ under $S U(2)_{I_{1}} \times S U(2)_{I_{2}}$ as can be seen from the commutations relations given below.

$$
\begin{array}{cl}
{\left[I_{1}^{i}, \Phi_{A}^{a}\right]=\frac{1}{2} \Phi_{A}^{b} \sigma_{b a}^{i}} & {\left[I_{1}^{i}, \Phi_{A}^{a \dagger}\right]=-\frac{1}{2} \sigma_{a b}^{i} \Phi_{A}^{b \dagger}} \\
{\left[I_{2}^{i}, \Psi^{a}\right]=0} & {\left[I_{2}^{i}, \bar{\Psi}^{a}\right]=0}
\end{array}
$$


We are interested in studying the states of the $\mathcal{N}=(4,4)$ SCFT on $\mathcal{M}$. The classification of the states and their symmetry properties can be analyzed by studying the states of a free field realization of a $\mathcal{N}=(4,4) \mathrm{SCFT}$ on $R^{4 Q_{1} Q_{5}} / S\left(Q_{1} Q_{5}\right)$. This is realized by considering the holomorphic and the anti-holomorphic $\mathcal{N}=4$ superconformal algebra with $c=\bar{c}=6 Q_{1} Q_{5}$ constructed out of $Q_{1} Q_{5}$ copies of four real fermions and bosons. So we have an anti-holomorphic component for each field, generator and charges discussed above. These are labelled by the same symbols used for the holomorphic components but distinguished by a tilde.

The charges $I_{1}, I_{2}$ constructed above generate $S O(4)$ transformations only on the holomorphic bosons $X_{A}(z)$. Similarly, we can construct charges $\widetilde{I}_{1}, \widetilde{I}_{2}$ which generate $S O(4)$ transformations only on the antiholomorphic bosons $\widetilde{X_{A}}(\bar{z})$. Normally one would expect these charges to give rise to a global $S O(4)_{h o l} \times S O(4)_{\text {antihol }}$ symmetry. However, the kinetic term of the bosons in the free field realization is not invariant under independent holomorphic and antiholomorphic $S O(4)$ rotations. It is easy to see, for example by using the Noether procedure, that there is a residual $S O(4)$ symmetry generated by the charges

$$
J_{I}=I_{1}+\widetilde{I}_{1} \quad \widetilde{J}_{I}=I_{2}+\widetilde{I}_{2}
$$

We will denote this symmetry as $S O(4)_{I}=S U(2)_{I} \times \widetilde{S U(2)_{I}}$, where the $S U(2)$ factors are generated by $J_{I}, \widetilde{J}_{I}$. These charges satisfy the property that (a) they correspond to $S O(4)$ transformations of the bosons $X_{A}(z, \bar{z})=X_{A}(z)+\widetilde{X_{A}}(\bar{z})$ and (b) they fall into representations of the $\mathcal{N}=(4,4)$ algebra (as can be proved by using the commutation relations (5.11) of the $I$ 's). The bosons $X(z, \bar{z})$ transform as $(\mathbf{2}, \mathbf{2})$ under $S U(2)_{I} \times S \widetilde{U(2)_{I}}$.

\subsection{The supergroup $S U(1,1 \mid 2)$}

The global part of the $\mathcal{N}=4$ superconformal algebra forms the supergroup $S U(1,1 \mid 2)$. Let $L_{ \pm, 0}, J_{R}^{(1),(2),(3)}$ be the global charges of the currents $T(z)$ and $J_{R}^{(i)}(z)$ and $G_{1 / 2,-1 / 2}^{a}$ the global charges of the supersymmetry currents $G^{a}(z)$ in the Neveu-Schwarz sector. From the OPE's (5.2) we obtain the following commutation relations for the global charges.

$$
\begin{array}{rc}
{\left[L_{0}, L_{ \pm}\right]=\mp L_{ \pm}} & {\left[L_{1}, L_{-1}\right]=2 L_{0}} \\
\left\{G_{1 / 2}^{a}, G_{-1 / 2}^{b \dagger}\right\}=2 \delta^{a b} L_{0}+2 \sigma_{a b}^{i} J_{R}^{(i)} \\
\left\{G_{-1 / 2}^{a}, G_{1 / 2}^{b \dagger}\right\}=2 \delta^{a b} L_{0}-2 \sigma_{a b}^{i} J_{R}^{(i)} \\
{\left[J_{R}^{(i)}, J_{R}^{(j)}\right]=i \epsilon^{i j k} J_{R}^{(k)}} \\
{\left[L_{0}, G_{ \pm 1 / 2}^{a}\right]=\mp \frac{1}{2} G_{ \pm 1 / 2}^{a}} & {\left[L_{0}, G_{ \pm 1 / 2}^{a \dagger}\right]=\mp \frac{1}{2} G_{ \pm 1 / 2}^{a \dagger}} \\
{\left[L_{+}, G_{1 / 2}^{a}\right]=0} & {\left[L_{-}, G_{-1 / 2}^{a}\right]=0} \\
{\left[L_{-}, G_{1 / 2}^{a}\right]=-G_{-1 / 2}^{a}} & {\left[L_{+}, G_{-1 / 2}^{a}\right]=G_{1 / 2}^{a}}
\end{array}
$$




$$
\begin{aligned}
{\left[L_{+}, G_{1 / 2}^{a \dagger}\right]=0 } & {\left[L_{-}, G_{1 / 2}^{a \dagger}\right]=0 } \\
{\left[L_{-}, G_{1 / 2}^{a \dagger}\right]=-G_{-1 / 2}^{a} } & {\left[L_{+}, G_{-1 / 2}^{a \dagger}\right]=G_{1 / 2}^{a} } \\
{\left[J_{R}^{(i)}, G_{ \pm 1 / 2}^{a}\right]=\frac{1}{2} G_{ \pm 1 / 2}^{b}\left(\sigma^{i}\right)^{b a} } & {\left[J_{R}^{(i)}, G_{ \pm 1 / 2}^{a \dagger}\right]=-\frac{1}{2}\left(\sigma^{i}\right)^{b a} G_{ \pm 1 / 2}^{b \dagger} }
\end{aligned}
$$

The above commutation relations form the algebra of the supergroup $S U(1,1 \mid 2)$. The global part of the $\mathcal{N}=(4,4)$ superconformal algebra form the super group $S U(1,1 \mid 2) \times S U(1,1 \mid 2)$.

\subsection{Short multiplets of $S U(1,1 \mid 2)$}

The representations of the supergroup $S U(1,1 \mid 2)$ are classified according to the conformal weight and $S U(2)_{R}$ quantum number. The highest weight states $|\mathrm{hw}\rangle=$ $\left|h, \mathbf{j}_{R}, j_{R}^{3}=j_{R}\right\rangle$ satisfy the following properties

$$
\begin{array}{rc}
L_{1}|\mathrm{hw}\rangle=0 & L_{0}|\mathrm{hw}\rangle=h|\mathrm{hw}\rangle \\
J_{R}^{(+)}|\mathrm{hw}\rangle=0 & J_{R}^{(3)}|\mathrm{hw}\rangle=j_{R}|\mathrm{hw}\rangle \\
G_{1 / 2}^{a}|\mathrm{hw}\rangle=0 & G_{1 / 2}^{a \dagger}|\mathrm{hw}\rangle=0
\end{array}
$$

where $J_{R}^{+}=J_{R}^{(1)}+i J_{R}^{(2)}$. Highest weight states which satisfy $G_{-1 / 2}^{2 \dagger}|\mathrm{hw}\rangle=0, \quad G_{-1 / 2}^{1}|\mathrm{hw}\rangle=$ 0 are chiral primaries. They satisfy $h=j$. We will denote these states as $|\mathrm{hw}\rangle_{S}$. Short multiplets are generated from the chiral primaries through the action of the raising operators $J_{-}, G_{-1 / 2}^{1 \dagger}$ and $G_{-1 / 2}^{2}$. The structure of the short multiplet is given below

$$
\begin{array}{cccc}
\text { States } & j & L_{0} & \text { Degeneracy } \\
|\mathrm{hw}\rangle_{S} & h & h & 2 h+1 \\
G_{-1 / 2}^{1 \dagger}|\mathrm{hw}\rangle_{S}, G_{-1 / 2}^{2}|\mathrm{hw}\rangle_{S} & h-1 / 2 & h+1 / 2 & 2 h+2 h=4 h \\
G_{-1 / 2}^{1 \dagger} G_{-1 / 2}^{2}|\mathrm{hw}\rangle_{S} & h-1 & h+1 & 2 h-1
\end{array}
$$

The short multiplets of the supergroup $S U(1,1 \mid 2) \times S U(1,1 \mid 2)$ are obtained by the tensor product of the above multiplet. We denote the short multiplet of $S U(1,1 \mid 2) \times S U(1,1 \mid 2)$ as $\left(\mathbf{2 h}+\mathbf{1}, \mathbf{2} \mathbf{h}^{\prime}+\mathbf{1}\right)_{\mathbf{s}}$. These stand for the degeneracy of the bottom component, the top row in (5.16). The top component of the short multiplet are the states belonging to the last row in (5.16). The short multiplet $(\mathbf{2}, \mathbf{2})_{\mathrm{S}}$ is special, it terminates at the middle row of (5.16). For this case, the top component is the middle row. These states have $h=\bar{h}=1$ and transform as $(\mathbf{1}, \mathbf{1})$ of $S U(2)_{R} \times S \widetilde{U(2)_{R}}$. There are 4 such states for each $(\mathbf{2}, \mathbf{2})_{\mathbf{S}}$.

\subsection{The resolutions of the symmetric product}

The Higgs branch of the D1-D5 system at low energies apart from the SCFT on the free torus $T^{4}$ is a $\mathrm{SCFT}$ on a resolution of the orbifold $\mathcal{M}$. So it is important 
for us to understand the operators corresponding to the moduli and the resolution of the orbifold $\mathcal{M}$. To this end we construct all the marginal operators of the $\mathcal{N}=(4,4) \mathrm{SCFT}$ on the symmetric product orbifold $\mathcal{M}$. We will find the four operators which correspond to resolution of the orbifold singularity.

\subsubsection{The untwisted sector}

Let us first focus on the operators constructed from the untwisted sector. The operators of lowest conformal weight are

$$
\begin{array}{ll}
\Psi_{A}^{1}(z) \widetilde{\Psi}_{A}^{1}(\bar{z}) & \Psi_{A}^{1}(z) \widetilde{\Psi}_{A}^{2 \dagger}(\bar{z}) \\
\Psi_{A}^{2 \dagger}(z) \widetilde{\Psi}_{A}^{1}(\bar{z}) & \Psi_{A}^{2 \dagger}(z) \widetilde{\Psi}_{A}^{2 \dagger}(\bar{z})
\end{array}
$$

where summation over $A$ is implied. These four operators have conformal dimension $(h, \bar{h})=(1 / 2,1 / 2)$ and $\left(j_{R}^{3}, \tilde{j}_{R}^{3}\right)=(1 / 2,1 / 2)$ under the R-symmetry $S U(2)_{R} \times S \widetilde{U U}(2)_{R}$. Since $(h, \bar{h})=\left(j_{R}^{3}, \widetilde{j}_{R}^{3}\right)$, these operators are chiral primaries and have non-singular operator product expansions (OPE) with the supersymmetry currents $G^{1}(z), G^{2 \dagger}(z), \widetilde{G}^{1}(\bar{z}), \widetilde{G}^{2 \dagger}(\bar{z})$. These properties indicate that they belong to the bottom component of the short multiplet $(\mathbf{2}, \mathbf{2})_{\mathrm{S}}{ }^{9}$. Each of the four chiral primaries gives rise to four top components of the short multiplet $(\mathbf{2}, \mathbf{2})_{\mathbf{S}}$. They are given by the leading pole $\left((z-w)^{-1}(\bar{z}-\bar{w})^{-1}\right)$ in the OPE's

$$
\begin{aligned}
G^{2}(z) \widetilde{G}^{2}(\bar{z}) \mathcal{P}(w, \bar{w}) & G^{2}(z) \widetilde{G}^{1 \dagger}(\bar{z}) \mathcal{P}(w, \bar{w}) \\
G^{1 \dagger}(z) \widetilde{G}^{2}(\bar{z}) \mathcal{P}(w, \bar{w}) & G^{1 \dagger}(z) \widetilde{G}^{1 \dagger}(\bar{z}) \mathcal{P}(w, \bar{w})
\end{aligned}
$$

where $\mathcal{P}$ stands for any of the four chiral primaries in (5.17). From the superconformal algebra it is easily seen that the top components constructed above have weights $(1,1)$ and transform as $(\mathbf{1}, \mathbf{1})$ under $S U(2)_{R} \times S \widetilde{U U}(2)_{R}$. The OPE's (5.18) can be easily evaluated. We find that the 16 top components of the $4(\mathbf{2}, \mathbf{2})_{\mathbf{S}}$ short multiplets are $\partial x_{A}^{i} \bar{\partial} x_{A}^{j}$.

We classify the above operators belonging to the top component according to representations of (a) the $S O(4)_{I}$ rotational symmetry of the $\widetilde{T}^{4}$, (The four torus $\tilde{T}^{4}$ breaks this symmetry but we assume the target space is $R^{4}$ for the classification of states) (b) $R$ symmetry of the SCFT and (c) the conformal weights. As all of these operators belong to the top component of $(\mathbf{2}, \mathbf{2})_{\mathrm{S}}$ the only property which distinguishes them is the representation under $S O(4)_{I}$. The quantum numbers of these operators under the various symmetries are

$$
\begin{array}{lccc}
\text { Operator } & S U(2)_{I} \times \widetilde{S U(2)_{I}} & S U(2)_{R} \times \widetilde{S U(2)_{R}}(h, \bar{h}) \\
\partial x_{A}^{\{i}(z) \bar{\partial} x_{A}^{j\}}(\bar{z})-\frac{1}{4} \delta^{i j} \partial x_{A}^{k}(z) \bar{\partial} x_{A}^{k}(\bar{z}) & (\mathbf{3}, \mathbf{3}) & (\mathbf{1}, \mathbf{1}) & (1,1) \\
\partial x_{A}^{[i}(z) \bar{\partial} x_{A}^{j]}(\bar{z}) & (\mathbf{3}, \mathbf{1})+(\mathbf{1}, \mathbf{3}) & (\mathbf{1}, \mathbf{1}) & (1,1) \\
\partial x_{A}^{i}(z) \bar{\partial} x_{A}^{i}(\bar{z}) & (\mathbf{1}, \mathbf{1}) & (\mathbf{1}, \mathbf{1}) & (1,1)
\end{array}
$$

\footnotetext{
${ }^{9}$ We restrict our operators to be single trace operators. This excludes operators of the type $\sum_{A=1}^{Q_{1} Q_{5}} \Psi_{A}^{1}(z) \sum_{B=1}^{Q_{1} Q_{5}} \tilde{\Psi}_{B}^{2 \dagger}(\bar{z})$. Multi-trace operators in the AdS/CFT correspondence has been discussed in 123, 124.
} 
Therefore we have 16 marginal operators from the untwisted sector. As these are top components they can be added to the free SCFT as perturbations without violating the $\mathcal{N}=(4,4)$ supersymmetry.

\subsection{2 $Z_{2}$ twists.}

We now construct the marginal operators from the various twisted sectors of the orbifold SCFT. The twist fields of the SCFT on the orbifold $\mathcal{M}$ are labeled by the conjugacy classes of the symmetric group $S\left(Q_{1} Q_{5}\right)$ [125, 126, 127]. The conjugacy classes consist of cyclic groups of various lengths. The various conjugacy classes and the multiplicity in which they occur in $S\left(Q_{1} Q_{5}\right)$ can be found from the solutions of the equation

$$
\sum n N_{n}=Q_{1} Q_{5}
$$

where $n$ is the length of the cycle and $N_{n}$ is the multiplicity of the cycle. Consider the simplest nontrivial conjugacy class which is given by $N_{1}=Q_{1} Q_{5}-2, N_{2}=1$ and the rest of $N_{n}=0$. A representative element of this class is

$$
\left(X_{1} \rightarrow X_{2}, X_{2} \rightarrow X_{1}\right), X_{3} \rightarrow X_{3}, \ldots, X_{Q_{1} Q_{5}} \rightarrow X_{Q_{1} Q_{5}}
$$

Here the $X_{A}$ 's are related to the $x_{A}$ 's appearing in the action (5.1) by (5.5).

To exhibit the singularity of this group action we go over to the following new coordinates

$$
X_{c m}=X_{1}+X_{2} \text { and } \phi=X_{1}-X_{2}
$$

Under the group action (5.21) $X_{c m}$ is invariant and $\phi \rightarrow-\phi$. Thus the singularity is locally of the type $R^{4} / Z_{2}$. The bosonic twist operators for this orbifold singularity are given by following OPE's [128]

$$
\begin{aligned}
& \partial \phi^{1}(z) \sigma^{1}(w, \bar{w})=\frac{\tau^{1}(w, \bar{w})}{(z-w)^{1 / 2}} \quad \partial \phi^{1 \dagger}(z) \sigma^{1}(w, \bar{w})=\frac{\tau^{11}(w, \bar{w})}{(z-w)^{1 / 2}} \\
& \partial \phi^{2}(z) \sigma^{2}(w, \bar{w})=\frac{\tau^{2}(w, \bar{w})}{(z-w)^{1 / 2}} \quad \partial \phi^{2 \dagger}(z) \sigma^{2}(w, \bar{w})=\frac{\tau^{\prime 2}(w, \bar{w})}{(z-w)^{1 / 2}} \\
& \bar{\partial} \widetilde{\phi}^{1}(\bar{z}) \sigma^{1}(w, \bar{w})=\frac{\widetilde{\tau}^{11}(w, \bar{w})}{(\bar{z}-\bar{w})^{1 / 2}} \quad \bar{\partial} \widetilde{\phi}^{1 \dagger}(\bar{z}) \sigma^{1}(w, \bar{w})=\frac{\widetilde{\tau}^{1}(w \bar{w})}{(\bar{z}-\bar{w})^{1 / 2}} \\
& \bar{\partial} \widetilde{\phi}^{2}(\bar{z}) \sigma^{2}(w, \bar{w})=\frac{\widetilde{\tau}^{2}(w, \bar{w})}{(\bar{z}-\bar{w})^{1 / 2}} \quad \bar{\partial} \widetilde{\phi}^{2 \dagger}(\bar{z}) \sigma^{2}(w, \bar{w})=\frac{\widetilde{\tau}^{2}(w, \bar{w})}{(\bar{z}-\bar{w})^{1 / 2}}
\end{aligned}
$$

The $\tau$ 's are excited twist operators. The fermionic twists are constructed from bosonized currents defined by

$$
\begin{array}{ll}
\chi^{1}(z)=e^{i H^{1}(z)} & \chi^{1 \dagger}(z)=e^{-i H^{1}(z)} \\
\chi^{2}(z)=e^{i H^{2}(z)} & \chi^{2 \dagger}(z)=e^{-i H^{2}(z)}
\end{array}
$$


Where the $\chi$ 's, defined as $\Psi_{1}-\Psi_{2}$, are the superpartners of the bosons $\phi$.

From the above we construct the supersymmetric twist fields which act both on fermions and bosons as follows:

$$
\begin{aligned}
\Sigma_{(12)}^{\left(\frac{1}{2}, \frac{1}{2}\right)} & =\sigma^{1}(z, \bar{z}) \sigma^{2}(z, \bar{z}) e^{i H^{1}(z) / 2} e^{-i H^{2}(z) / 2} e^{i \widetilde{H}^{1}(\bar{z}) / 2} e^{-i \widetilde{H}^{2}(\bar{z}) / 2} \\
\Sigma_{(12)}^{\left(\frac{1}{2},-\frac{1}{2}\right)} & =\sigma^{1}(z, \bar{z}) \sigma^{2}(z, \bar{z}) e^{i H^{1}(z) / 2} e^{-i H^{2}(z) / 2} e^{-i \widetilde{H}^{1}(\bar{z}) / 2} e^{i \widetilde{H}^{2}(\bar{z}) / 2} \\
\Sigma_{(12)}^{\left(-\frac{1}{2}, \frac{1}{2}\right)} & =\sigma^{1}(z, \bar{z}) \sigma^{2}(z, \bar{z}) e^{-i H^{1}(z) / 2} e^{+i H^{2}(z) / 2} e^{i \widetilde{H}^{1}(\bar{z}) / 2} e^{-i \widetilde{H}^{2}(\bar{z}) / 2} \\
\Sigma_{(12)}^{\left(-\frac{1}{2},-\frac{1}{2}\right)} & =\sigma^{1}(z, \bar{z}) \sigma^{2}(z, \bar{z}) e^{-i H^{1}(z) / 2} e^{+i H^{2}(z) / 2} e^{-i \widetilde{H}^{1}(\bar{z}) / 2} e^{+i \widetilde{H}^{2}(\bar{z}) / 2}
\end{aligned}
$$

The subscript (12) refers to the fact that these twist operators were constructed for the representative group element (5.21) which exchanges the 1 and 2 labels of the coordinates of $\widetilde{T}^{4}$. The superscript stands for the $\left(j_{R}^{3}, \widetilde{j}_{R}^{3}\right)$ quantum numbers. The twist operators for the orbifold $\mathcal{M}$ belonging to the conjugacy class under consideration is obtained by summing over these $Z_{2}$ twist operators for all representative elements of this class.

$$
\Sigma^{\left(\frac{1}{2}, \frac{1}{2}\right)}=\sum_{i=1}^{Q_{1} Q_{5}} \sum_{j=1, j \neq i}^{Q_{1} Q_{5}} \Sigma_{(i j)}^{\left(\frac{1}{2}, \frac{1}{2}\right)}
$$

We can define the rest of the twist operators for the orbifold in a similar manner. The conformal dimensions of these operators are $(1 / 2,1 / 2)$. They transform as $(\mathbf{2}, \mathbf{2})$ under the $S U(2)_{R} \times \widetilde{S U(2)_{R}}$ symmetry of the SCFT. They belong to the bottom component of the short multiplet $(\mathbf{2}, \mathbf{2})_{\mathbf{S}}$. The operator $\Sigma^{\left(\frac{1}{2}, \frac{1}{2}\right)}$ is a chiral primary. As before the 4 top components of this short multiplet, which we denote by

$$
\begin{array}{r}
T^{\left(\frac{1}{2}, \frac{1}{2}\right)}, \quad T^{\left(\frac{1}{2},-\frac{1}{2}\right)} \\
T^{\left(-\frac{1}{2}, \frac{1}{2}\right)}, \quad T^{\left(-\frac{1}{2},-\frac{1}{2}\right)}
\end{array}
$$

are given by the leading pole in the following OPE's respectively

$$
\begin{array}{ll}
G^{2}(z) \widetilde{G}^{2}(\bar{z}) \Sigma^{\left(\frac{1}{2}, \frac{1}{2}\right)}(w, \bar{w}), & G^{2}(z) \widetilde{G}^{1 \dagger}(\bar{z}) \Sigma^{\left(\frac{1}{2}, \frac{1}{2}\right)}(w, \bar{w}), \\
G^{1 \dagger}(z) \widetilde{G}^{2}(\bar{z}) \Sigma^{\left(\frac{1}{2}, \frac{1}{2}\right)}(w, \bar{w}), & G^{1 \dagger}(z) \widetilde{G}^{1 \dagger}(\bar{z}) \Sigma^{\left(\frac{1}{2}, \frac{1}{2}\right)}(w, \bar{w})
\end{array}
$$

These are the 4 blow up modes of the $R^{4} / Z_{2}$ singularity 129 and they have conformal weight $(1,1)^{10}$. They transform as $(\mathbf{1}, \mathbf{1})$ under the $S U(2)_{R} \times S \widetilde{S(2)_{R}}$. As before, since these are top components of the short multiplet $(\mathbf{2}, \mathbf{2})_{\mathrm{S}}$ they can be added to the free SCFT as perturbations without violating the $\mathcal{N}=(4,4)$

\footnotetext{
${ }^{10}$ Relevance of $Z_{2}$ twist operators to the marginal deformations of the SCFT has earlier been discussed in 130, 131.
} 
supersymmetry of the SCFT. The various quantum numbers of these operators are listed below.

$$
\begin{array}{lccc}
\text { Operator } & \left(j^{3}, \tilde{j}^{3}\right)_{I} & S U(2)_{R} \times \widetilde{S U(2)_{R}} & (h, \bar{h}) \\
\mathcal{T}_{(1)}^{1}=T^{\left(\frac{1}{2}, \frac{1}{2}\right)} & (0,1) & (\mathbf{1}, \mathbf{1}) & (1,1) \\
\mathcal{T}_{(0)}^{1}=T^{\left(\frac{1}{2},-\frac{1}{2}\right)}+T^{\left(-\frac{1}{2}, \frac{1}{2}\right)} & (0,0) & (\mathbf{1}, \mathbf{1}) & (1,1) \\
\mathcal{T}_{(-1)}^{1}=T^{\left(-\frac{1}{2},-\frac{1}{2}\right)} & (0,-1) & (\mathbf{1}, \mathbf{1}) & (1,1) \\
\mathcal{T}^{0}=T^{\left(-\frac{1}{2},-\frac{1}{2}\right)}-T^{\left(-\frac{1}{2},-\frac{1}{2}\right)} & (0,0) & (\mathbf{1}, \mathbf{1}) & (1,1)
\end{array}
$$

The first three operators of the above table can be organized as a $(\mathbf{1}, \mathbf{3})$ under $S U(2)_{I} \times \widetilde{S U(2)_{I}}$. We will denote these 3 operators as $\mathcal{T}^{1}$. The last operator transforms as a scalar $(\mathbf{1}, \mathbf{1})$ under $S U(2)_{I} \times \widehat{S U(2)_{I}}$ and is denoted by $\mathcal{T}^{0}$. The simplest way of figuring out the $\left(j^{3}, \tilde{j}^{3}\right)_{I}$ quantum numbers in the above table is to note that (a) the $\Sigma$-operators of (5.25) are singlets under $S U(2)_{I} \times \widetilde{S U(2)_{I}}$, as can be verified by computing the action on them of the operators $I_{1}, I_{2}$ and $\widetilde{I}_{1}, \widetilde{I}_{2}$, (b) the $\mathcal{T}$-operators are obtained from $\Sigma$ 's by the action of the supersymmetry currents as in (5.28) and (c) the quantum numbers of the supersymmetry currents under $I_{1}, I_{2}$ and $\widetilde{I}_{1}, \widetilde{I}_{2}$ are given by $(5.9)$.

\subsubsection{Higher twists}

We now show that the twist operators corresponding to any other conjugacy class of $S\left(Q_{1} Q_{5}\right)$ are irrelevant. Consider the class with $N_{1}=Q_{1} Q_{5}-3, N_{3}=1$ and the rest of $N_{n}=0$. A representative element of this class is

$$
\left(X_{1} \rightarrow X_{2}, X_{2} \rightarrow X_{3}, X_{3} \rightarrow X_{1}\right), X_{4} \rightarrow X_{4}, \ldots, X_{Q_{1} Q_{5}} \rightarrow X_{Q_{1} Q_{5}} .
$$

To make the action of this group element transparent we diagonalize the group action as follows.

$$
\left(\begin{array}{l}
\phi_{1} \\
\phi_{2} \\
\phi_{3}
\end{array}\right)=\left(\begin{array}{ccc}
1 & 1 & 1 \\
1 & \omega & \omega^{2} \\
1 & \omega^{2} & \omega^{4}
\end{array}\right)\left(\begin{array}{l}
X_{1} \\
X_{2} \\
X_{3}
\end{array}\right)
$$

where $\omega=\exp (2 \pi i / 3)$. These new coordinates are identified under the group action (5.30) $\phi_{1} \rightarrow \phi_{1}, \phi_{2} \rightarrow \omega^{2} \phi_{2}$ and $\phi_{3} \rightarrow \omega \phi_{3}$. These identifications are locally characteristic of the orbifold

$$
R^{4} \times R^{4} / \omega \times R^{4} / \omega^{2}
$$

The dimension of the supersymmetric twist operator which twists the coordinates by a phase $e^{2 \pi i k / N}$ in 2 complex dimensions is $h(k, N)=k / N[128]$. The twist operator which implements the action of the group element (5.30) combines the supersymmetric twist operators acting on $\phi_{2}$ and $\phi_{3}$ and therefore has total dimension

$$
h=h(1,3)+h(2,3)=1 / 3+2 / 3=1
$$


It is the superpartners of these which could be candidates for the blow up modes. However, these have weight $3 / 2$, These operators are therefore irrelevant.

For the class $N_{1}=Q_{1} Q_{5}-k, N_{k}=k$ and the rest of $N_{n}=0$, the total dimension of the twist operator is

$$
h=\sum_{i=1}^{k-1} h(i, k)=(k-1) / 2
$$

Its superpartner has dimension $k / 2$. Now it is easy to see that all conjugacy classes other than the exchange of 2 elements give rise to irrelevant twist operators. Thus the orbifold $\mathcal{M}$ is resolved by the 4 blow up modes corresponding to the conjugacy class represented by (5.21). We have thus identified the 20 marginal operators of the $\mathcal{N}=(4,4) \mathrm{SCFT}$ on $\widetilde{T}^{4}$. They are all top components of the $5(\mathbf{2}, \mathbf{2})_{\mathbf{S}}$ short multiplets. The $5(\mathbf{2}, \mathbf{2})_{\mathrm{S}}$ have 20 operators of conformal dimensions $(h, \bar{h})=$ $(1 / 2,1 / 2)$. These are relevant operators for the SCFT. It would be interesting to investigate the role of these relevant operators. As they are chiral primaries they would break only half of the supersymmetries of the SCFT and therfore the renormalization group flow induced by these operators would persumably be tractable for study.

\subsection{The chiral primaries of the $\mathcal{N}=(4,4)$ SCFT on $\mathcal{M}$}

In this section we will explicitly construct all the chiral primaries corresponding to single particle states of the SCFT on the orbifold $\mathcal{M}$. For this purpose we will have to construct the twist operator corresponding to the conjugacy class $N_{1}=Q_{1} Q_{5}-k, N_{k}=k$ and the rest of $N_{n}=0$.

\subsubsection{The k-cycle twist operator}

We will extend the method of construction of the 2-cycle twist operator of Section 5.6 .2 to the construction of the k-cycle twist operator. Consider the conjugacy class given by $N_{1}=Q_{1} Q_{5}-k, N_{k}=k$ and the rest of $N_{n}=0$. A representative element of this class is the following group action

$$
\left(X_{1} \rightarrow X_{2}, \ldots, X_{k} \rightarrow X_{1}\right), X_{k+1} \rightarrow X_{k+1}, \ldots, X_{Q_{1} Q_{5}} \rightarrow X_{Q_{1} Q_{5}}
$$

We can diagonalize the group action as follows

$$
\left(\begin{array}{c}
\phi_{k} \\
\phi_{k-1} \\
\phi_{k-2} \\
\vdots \\
\phi_{1}
\end{array}\right)=\left(\begin{array}{ccccc}
1 & 1 & 1 & \ldots & 1 \\
1 & \omega & \omega^{2} & \ldots & \omega^{k-1} \\
1 & \omega^{2} & \omega^{4} & \ldots & \omega^{2(k-1)} \\
\vdots & \vdots & \vdots & \ldots & \vdots \\
1 & \omega^{k-1} & \omega^{(k-1) 2} & \ldots & \omega^{(k-1)(k-1)}
\end{array}\right)\left(\begin{array}{c}
X_{1} \\
X_{2} \\
X_{3} \\
\vdots \\
X_{k}
\end{array}\right)
$$


where $\omega=e^{2 \pi i / k}$. These new coordinates are identified under the group action (5.35) as

$$
\phi_{1} \rightarrow \omega \phi_{1}, \quad \phi_{2} \rightarrow \omega^{2} \phi_{2}, \quad \phi_{3} \rightarrow \omega^{3} \phi_{3}, \quad \ldots, \quad \phi_{k-1} \rightarrow \omega^{k-1} \phi_{k-1}, \quad \phi_{k} \rightarrow \omega^{k} \phi_{k}
$$

These identifications are locally characteristic of the orbifold

$$
R^{4} \times R^{4} / \omega \times R^{4} / \omega^{2} \times \ldots \times R^{4} / \omega^{k-1}
$$

The coordinate $\phi_{m}$ is twisted by the phase $\omega^{m}$ ( $m$ runs from $1 \ldots k$ ). The bosonic twist operators corresponding to this twist are defined by the following OPE's

$$
\begin{aligned}
\partial \phi_{m}^{1}(z) \sigma_{m}^{1}(w, \bar{w})=\frac{\tau_{m}^{1}(w, \bar{w})}{(z-w)^{1-m / k}} & \partial \phi_{m}^{1 \dagger}(z) \sigma_{m}^{1}(w, \bar{w})=\frac{\tau_{m}^{\prime 1}(w, \bar{w})}{(z-w)^{m / k}} \\
\partial \phi_{m}^{2}(z) \sigma_{m}^{2}(w, \bar{w})=\frac{\tau_{m}^{2}(w, \bar{w})}{(z-w)^{1-m / k}} & \partial \phi_{m}^{2 \dagger}(z) \sigma_{m}^{2}(w, \bar{w})=\frac{\tau_{m}^{\prime 2}(w, \bar{w})}{(z-w)^{m / k}} \\
\bar{\partial} \widetilde{\phi}_{m}^{1}(\bar{z}) \sigma_{m}^{1}(w, \bar{w})=\frac{\widetilde{\tau}_{m}^{\prime 1}(w, \bar{w})}{(\bar{z}-\bar{w})^{m / k}} & \bar{\partial} \widetilde{\phi}_{m}^{1 \dagger}(\bar{z}) \sigma_{m}^{1}(w, \bar{w})=\frac{\widetilde{\tau}_{m}^{1}(w, \bar{w})}{(\bar{z}-\bar{w})^{1-m / k}} \\
\bar{\partial} \widetilde{\phi}_{m}^{2}(\bar{z}) \sigma_{m}^{2}(w, \bar{w})=\frac{\widetilde{\tau}_{m}^{\prime 2}(w, \bar{w})}{(\bar{z}-\bar{w})^{m / k}} & \bar{\partial} \widetilde{\phi}_{m}^{2 \dagger}(\bar{z}) \sigma_{m}^{2}(w, \bar{w})=\frac{\widetilde{\tau}_{m}^{2}(w, \bar{w})}{(\bar{z}-\bar{w})^{1-m / k}}
\end{aligned}
$$

As in Section 5.6.2 $\tau$ 's are excited twist operators. The fermionic twists are constructed from bosonized currents defined by

$$
\begin{array}{ll}
\chi_{m}^{1}(z)=e^{i H_{m}^{1}(z)} & \chi_{m}^{1 \dagger}(z)=e^{-i H_{m}^{1}(z)} \\
\chi_{m}^{2}(z)=e^{i H_{m}^{2}(z)} & \chi_{m}^{2 \dagger}(z)=e^{-i H_{m}^{2}(z)}
\end{array}
$$

Where the $\chi_{m}$ 's are the superpartners of the bosons $\phi_{m}$ 's. The twist operators corresponding to the fermions $\chi_{m}$ 's are given by $e^{ \pm i m H_{m} / k}$.

We now assemble all these operators to construct the k-cycle twist operator which is a chiral primary. The k-cycle twist operator is given by

$$
\Sigma_{(12 \ldots k)}^{(k-1) / 2}=\prod_{m=1}^{k-1}\left[\sigma_{m}^{1}(z, \bar{z}) \sigma_{m}^{2}(z, \bar{z}) e^{i m H_{m}^{1}(z) / k} e^{-i m H_{m}^{2}(z) / k} e^{i m \tilde{H}_{m}^{1}(\bar{z}) / k} e^{-i m \tilde{H}_{m}^{2}(\bar{z}) / k}\right]
$$

The subscript $(12 \ldots k)$ refers to the fact that these twist operators were constructed for the representative group element (5.35) which cyclically permutes the $1, \ldots, k$ labels of the coordinates of $\tilde{T}^{4}$. The superscript $(k-1) / 2$ stands for the conformal dimension of this operator. As we saw in Section 5.6.3 the conformal dimension of the twist operator for the conjugacy class $N_{1}=Q_{1} Q_{5}-k, N_{k}=k$ and the rest of $N_{n}=0$ is $(h, \bar{h})=((k-1) / 2,(k-1) / 2)$. The twist operator for 
the conjugacy class under consideration is obtained by summing over the k-cycle twist operators for all representative element of these class.

$$
\Sigma^{(k-1) / 2}(z, \bar{z})=\sum_{\left\{i_{i}, \ldots, i_{k}\right\}} \Sigma_{i_{1} i_{2} \ldots i_{k}}(z, \bar{z})
$$

where the sum runs over all $k$-tuples $\left\{i_{i} \ldots, i_{k}\right\}$ such that $i_{i} \neq i_{2} \neq \ldots \neq i_{k}$. $i_{m}$ take values from 1 to $Q_{1} Q_{5}$. The operator $\Sigma^{(k-1) / 2}$ is a chiral primary with conformal dimension $(h, \bar{h})=((k-1) / 2,(k-1) / 2)$ and $\left(j_{R}^{3}, \tilde{j}_{R}^{3}\right)=((k-1) / 2,(k-$ $1) / 2$ ). As the largest cycle is of length $Q_{1} Q_{5}$, the maximal dimension of the $\mathrm{k}$ cycle twist operator is $\left(\left(Q_{1} Q_{5}-1\right) / 2,\left(Q_{1} Q_{5}-1\right) / 2\right)$. It belongs to the bottom component of the short multiplet $(\mathbf{k}, \mathbf{k})_{\mathbf{s}}$. The other components of the short multiplet $(\mathbf{k}, \mathbf{k})_{\mathbf{S}}$ corresponding to the k-cycle twists can be generated by the action of supersymmetry currents and the R-symmetry currents of the $\mathcal{N}=(4,4)$ theory on $\mathcal{M}$.

\subsubsection{The complete set of chiral primaries}

We have seen is Section 5.6 there are five chiral primaries corresponding to the short multiplet $5(\mathbf{2}, \mathbf{2})_{\mathbf{S}}$. In this section we will construct the complete set of chiral primaries from single particle states of the SCFT on $\mathcal{M}$. It is known that the chiral primaries with weight $(h, \bar{h})$ of a $\mathcal{N}=(4,4)$ superconformal field theory on a manifold $K$ correspond to the elements of the cohomology $\mathcal{H}_{2 h 2 \bar{h}}(K)$ [132]. The chiral primaries are formed by the product of the chiral primaries corresponding to the cohomology of the diagonal $\tilde{T}^{4}$ denoted by $B^{4}$ (the sum of all copies of $\tilde{T}^{4}$ ) and the various k-cycle chiral primaries constructed in Section 5.7.1. We will list the chiral primaries below

\section{Chiral primaries with $h-\bar{h}=0$}

All the k-cycle chiral primaries have $h-\bar{h}=0$. To construct chiral primaries with $h-\bar{h}=0$ we need the four chiral primaries which correspond to the cohomology $\mathcal{H}_{11}\left(B^{4}\right)$ with weight $(1 / 2,1 / 2)$. They are given in (5.17). Using this we can construct the following chiral primaries

$$
\begin{array}{ll}
\Sigma^{(k-1) / 2}(z, \bar{z}) \Psi_{A}^{1}(z) \widetilde{\Psi}_{A}^{1}(\bar{z}) & \Sigma^{(k-1) / 2}(z, \bar{z}) \Psi_{A}^{1}(z) \widetilde{\Psi}_{A}^{2 \dagger}(\bar{z}) \\
\Sigma^{(k-1) / 2}(z, \bar{z}) \Psi_{A}^{2 \dagger}(z) \widetilde{\Psi}_{A}^{1}(\bar{z}) & \Sigma^{(k-1) / 2}(z, \bar{z}) \Psi_{A}^{2 \dagger}(z) \widetilde{\Psi}_{A}^{2 \dagger}(\bar{z})
\end{array}
$$

where summation over $A$ is implied. These four operators have conformal dimension $(k / 2, k / 2)$. There is one more chiral primary corresponding to the cohomology $\mathcal{H}_{22}\left(B^{4}\right)$ for which $h-\bar{h}=0$. It is given by

$$
\Psi_{A}^{1}(z) \Psi_{A}^{2 \dagger}(z) \widetilde{\Psi}_{A}^{1}(\bar{z}) \widetilde{\Psi}_{A}^{2 \dagger}(\bar{z})
$$

where summation over all indices of $A$ is implied. This chiral primary corresponds to the top form of $B^{4}$. The cohomology $\mathcal{H}_{00}\left(B^{4}\right)$ gives rise to a chiral primaries of 
conformal dimension $(k / 2, k / 2)$. It is given by

$$
\Sigma^{(k-2) / 2}(z, \bar{z}) \Psi_{A}^{1}(z) \Psi_{A}^{2 \dagger}(z) \widetilde{\Psi}_{A}^{1}(\bar{z}) \widetilde{\Psi}_{A}^{2 \dagger}(\bar{z})
$$

From the equation above we see that these chiral primaries exist only of $k \geq 2$. Finally we have the chiral primary $\Sigma^{(k) / 2}(z, \bar{z})$ of conformal dimension $(k / 2, k / 2)$. Thus for $k \geq 2$ and $k \leq Q_{1} Q_{5}-1$ there are 6 chiral primaries of dimension $(k / 2, k / 2)$

The complete list of chiral primaries with $(h, \bar{h})$ with $h-\bar{h}=0$ corresponding to single particle states are given by

$\begin{array}{lc}(h, \bar{h}) & \text { Degeneracy } \\ (1 / 2,1 / 2) & 5 \\ (1,1) & 6 \\ (3 / 2,3 / 2) & 6 \\ \vdots & \vdots \\ \left(\left(Q_{1} Q_{5}-1\right) / 2,\left(Q_{1} Q_{5}-1\right) / 2\right) & 6 \\ \left(\left(Q_{1} Q_{5}\right) 2,\left(Q_{1} Q_{1}\right) / 2\right) & 5 \\ \left(\left(Q_{1} Q_{5}+1\right) / 2,\left(Q_{1} Q_{5}+1\right) / 2\right) & 1\end{array}$

In the above table we have ignored the vacuum with weight $(h, \bar{h})=(0,0)$.

Chiral primaries with $h-\bar{h}=1 / 2$

The chiral primaries of $B^{4}$ which correspond to the elements of the cohomology $\mathcal{H}_{10}\left(B^{4}\right)$ are given by

$$
\sum_{A=1}^{Q_{1} Q_{5}} \Psi_{A}^{1}(z) \text { and } \sum_{A=1}^{Q_{1} Q_{5}} \Psi_{A}^{2 \dagger}(z)
$$

We can construct chiral primaries with weight $((k+1) / 2, k / 2))$ by taking the product of the above chiral primaries with the twist operator $\Sigma^{k / 2}(z, \bar{z})$. These give the following chiral primaries

$$
\Sigma^{k / 2}(z, \bar{z}) \sum_{A=1}^{Q_{1} Q_{5}} \Psi_{A}^{1}(z) \quad \text { and } \quad \Sigma^{k / 2}(z, \bar{z}) \sum_{A=1}^{Q_{1} Q_{5}} \Psi_{A}^{2 \dagger}(z)
$$

The chiral primary of the diagonal $B^{4}$ which correspond to the elements of the cohomology $\mathcal{H}_{21}\left(B^{4}\right)$ are

$$
\Psi_{A}^{1}(z) \Psi_{A}^{2 \dagger}(z) \widetilde{\Psi}_{A}^{1}(\bar{z}) \text { and } \Psi_{A}^{1}(z) \Psi_{A}^{2 \dagger}(z) \widetilde{\Psi}_{A}^{2 \dagger}(\bar{z})
$$

Here summation over all the three indices of $A$ is implied. From these the one can construct chiral primaries with weight $((k+1) / 2, k / 2)$ are follows

$$
\Sigma^{(k-1) / 2}(z, \bar{z}) \Psi_{A}^{1}(z) \Psi_{A}^{2 \dagger}(z) \widetilde{\Psi}_{A}^{1}(\bar{z}) \text { and } \Sigma^{(k-1) / 2}(z, \bar{z}) \Psi_{A}^{1}(z) \Psi_{A}^{2 \dagger}(z) \widetilde{\Psi}_{A}^{2 \dagger}(\bar{z})
$$


Therefore there are 4 chiral primaries with weight $((k+1) / 2, k / 2)$ for $1 \leq k \leq$ $\left(Q_{1} Q_{5}-1\right)$ and 2 chiral primaries with weight $\left(\left(Q_{1} Q_{5}+1\right) / 2, Q_{1} Q_{5} / 2\right)$. There are also 2 chiral primaries with weight $(1 / 2,0)$.

Chiral primaries with $\bar{h}-h=1 / 2$

The procedure for constructing these chiral primaries are identical to the case $h-\bar{h}=1 / 2$. The four chiral primaries with weight $(k / 2,(k+1) / 2)$ are given by

$$
\begin{aligned}
\Sigma^{k / 2}(z, \bar{z}) \sum_{A=1}^{Q_{1} Q_{5}} \widetilde{\Psi}_{A}^{1}(\bar{z}) & \Sigma^{k / 2}(z, \bar{z}) \sum_{A=1}^{Q_{1} Q_{5}} \widetilde{\Psi}_{A}^{2 \dagger}(\bar{z}) \\
\Sigma^{(k-1) / 2}(z, \bar{z}) \Psi_{A}^{1}(z) \widetilde{\Psi}_{A}^{1}(\bar{z}) \widetilde{\Psi}_{A}^{2 \dagger}(\bar{z}) & \Sigma^{(k-1) / 2}(z, \bar{z}) \Psi_{A}^{2 \dagger}(z) \widetilde{\Psi}_{A}^{1}(\bar{z}) \widetilde{\Psi}_{A}^{2 \dagger}(\bar{z})
\end{aligned}
$$

There are 4 chiral primaries with weight $(k / 2,(k+1) / 2)$ for $1 \leq k \leq\left(Q_{1} Q_{5}-1\right)$ 2 chiral primaries with weight $\left(Q_{1} Q_{5} / 2,\left(Q_{1} Q_{5}+1\right) / 2\right)$ and 2 chiral primaries with weight $(0,1 / 2)$.

Chiral primaries with $h-\bar{h}=1$

As in the previous cases let us first look at the chiral primaries corresponding to the cohomology element $\mathcal{H}_{20}\left(B^{4}\right)$. There is only one element which is given by

$$
\Psi_{A}^{1}(z) \Psi_{A}^{2 \dagger}(z)
$$

where summation over $A$ is implied. There is a single chiral primary with weight $((k+2) / 2, k / 2)$ constructed out of the above chiral primary is

$$
\Sigma^{k / 2}(z, \bar{z}) \Psi_{A}^{1}(z) \Psi_{A}^{2 \dagger}(z)
$$

Thus there is a one chiral primary with weight $((k+2) / 2, k / 2)$ for $0 \leq k \leq$ $\left(Q_{1} Q_{5}-1\right)$. The operator product expansion of two chiral primaries will give rise to other chiral primaries consistent with conservation laws. There are known to form a ring. It will be interesting to understand the structrure of this ring.

Chiral primaries with $\bar{h}-h=1$

The construction of these is parallel to the case for $h-\bar{h}=1$. The single chiral primary with weight $(k / 2,(k+2) / 2)$ for $0 \leq k \leq\left(Q_{1} Q_{5}-1\right)$ is given by

$$
\Sigma^{k / 2}(z, \bar{z}) \widetilde{\Psi}_{A}^{1}(\bar{z}) \widetilde{\Psi}_{A}^{2 \dagger}(\bar{z})
$$

There are no chiral primaries with $h-\bar{h}>1$ or $\bar{h}-h>1$. From the construction of the chiral primaries we see that such chiral primaries can exist only if there is an element in $\mathcal{H}_{r 0}\left(B^{2}\right)$ or $\mathcal{H}_{0 r}\left(B^{2}\right)$ with $r>1$. As the homology groups of $B^{4}$ is identical to that of a four torus we know that such elements do not exist. 


\subsection{Short multiplets of $\mathcal{N}=(4,4)$ SCFT on $\mathcal{M}$}

Using the results of Section 5.7 we will write the complete set of short multiplets of single particle states of the $\mathcal{N}=(4,4) \mathrm{SCFT}$ onf $\mathcal{M}$. In Section 6 we will compare this set of short multiplets with that obtained from supergravity. We will see in Section 6.1 that supergravity is a good approximation in string theory only when $Q_{1} \rightarrow \infty, Q_{5} \rightarrow \infty$. Therefore we write down the list of short multiplets for $\left(\tilde{T}^{4}\right)^{(\infty)} / S(\infty)$. Basically this means that the list of chiral primaries of the previous Section 5.7 does not terminate.

We have seen that each chiral primary of weight $\left(h, h^{\prime}\right)$ gives rise gives rise to the short multiplet $\left.\left(\mathbf{2 h}+\mathbf{1}, \mathbf{2} \mathbf{h}^{\prime}+\mathbf{1}\right)\right)_{S}$. Therefore the results of Section 5.7 indicate that the list of shormultiplets corresponding to the single particle states of $\mathcal{N}=(4,4)$ SCFT on $\left(\tilde{T}^{4}\right)^{(\infty)} / S(\infty)$. is given by

$$
\begin{gathered}
5(\mathbf{2}, \mathbf{2})_{S}+6 \oplus_{\mathbf{m} \geq \mathbf{3}}(\mathbf{m}, \mathbf{m})_{S} \\
2(\mathbf{1}, \mathbf{2})_{S}+2(\mathbf{2}, \mathbf{1})_{S}+(\mathbf{1}, \mathbf{3})_{S}+(\mathbf{3}, \mathbf{1})_{S} \\
\oplus_{\mathbf{m} \geq \mathbf{2}}\left[(\mathbf{m}, \mathbf{m}+\mathbf{2})_{S}+(\mathbf{m}+\mathbf{2}, \mathbf{m})_{S}+4(\mathbf{m}, \mathbf{m}+\mathbf{1})_{S}+4(\mathbf{m}+\mathbf{1}, \mathbf{m})_{S}\right]
\end{gathered}
$$

In our discussion so far we have ignored the short multiplets from the free torus $T^{4}$ which forms a part of the Higgs branch of the D1-D5 system. We will see in section 6 that the short multiplets from the free torus are not present in the supergravity. Thus for comparision with supergravity it is sufficient for us to restrict our attention to the shortmultiplets on $\mathcal{M}$.

\subsection{Stringy exclusion principle}

We see from the preceding discussion that the spin of short multiplets in the SCFT is bounded by $\left(1+Q_{1} Q_{5}\right) / 2$. In the context of the $\mathrm{AdS}_{3} / \mathrm{CFT}_{2}$ correspondence (see Section 6) this is puzzling at first since there is no corresponding bound on spin from supergravity. However, since supergravity is only valid at $Q_{1}, Q_{5} \rightarrow \infty$ these two facts are reconciled. The existence of a maximum spin for finite $Q_{1}, Q_{5}$ has been called the "stringy exclusion principle" 90]. Clearly this bound cannot be understood in supergravity and should be understood in terms an exact treatment of strings in $\mathrm{AdS}_{3}$. 


\section{Near horizon supergravity and SCFT}

In this section we will classify the supergravity fields according to the symmetries of the near horizon geometry of the D1-D5 system which was derived in section. 2.6 and compare them with the chiral multiplets of the SCFT on $\mathcal{M}$.

Let us examine the symmetries of the near horizon geometry (2.70). The bosonic symmetries arise from the isometries of $A d S_{3}$ and $S^{3}$. The isometries of the $A d S_{3}$ space form the non-compact group $S O(2,2)$, while the isometries of $S^{3}$ form the group $S O(4)_{E}=S U(2)_{E} \times S \widetilde{U(2)_{E}}$. Though the compactification on $T^{4}$ breaks the $S O(4)$ rotations of the coordinates $x_{6}, \ldots, x_{9}$ we can still use this symmetry to classify supergravity fields. We will call this symmetry $S O(4)_{I}$. The D1-D5 system preserves eight out of the 32 supersymmetries of the type IIB theory. In the near horizon limit the number of supersymmetries gets enhanced from eight to sixteen [133, 134]. These symmetries fix the form of the effective anti-de Sitter supergravity theory near the horizon. The bosonic symmetries $S O(2,2) \times S O(4)_{E}=(S L(2, R) \times S U(2)) \times(S L(2, R) \times S U(2))$ form the bosonic symmetries of the anti-de Sitter supergravity in three-dimensions. Simple anti-de Sitter supergroups in three-dimensions were classified in [135]. It can be seen that the only simple supergroups whose bosonic part is $S L(2, R) \times S U(2)$ are $O s p(3 \mid 2, R)$ and $S U(1,1 \mid 2)$. The former contains the bosonic subgroup $O(3) \times S L(2, R)$. The supercharges of the supergroup $O s p(3 \mid 2, R)$ transform as the vector representation of the group $O(3)$, while the supercharges of the supergroup $S U(1,1 \mid 2)$ transform as 2 of the group $S U(2)$. The unbroken supercharges of the D1-D5 system transform in the spinor representation of $S O(4)_{E}$ and therefore they transform as $\mathbf{2}$ of $S U(2)$. This rules out $O s p(3 \mid 2, R)$. Therefore the near horizon anti-de Sitter supergravity is based on the supergroup $S U(1,1 \mid 2) \times S U(1,1 \mid 2)$ with matter fields 11.

\subsection{Classification of the supergravity modes}

In this section we analyze the spectrum of Type IIB supergravity compactified on $A d S_{3} \times S^{3} \times T^{4}$. From (2.72) we see the volume of $T^{4}$ is $16 \pi^{4} \alpha^{\prime 2} Q_{1} / Q_{5}$. Therefore we ignore Kaluza-Klein modes on the $T^{4}$. The radius of the $S^{3}$ is $\sqrt{\alpha^{\prime}}\left(g_{6} Q_{1} Q_{5}\right)^{1 / 4}$. This is large when

$$
g_{s} Q_{1}>>1 \text { and } g_{s} Q_{5}>>1
$$

These inequalities imply that we are working in the regime where closed string perturbation theory is valid and where all length scales are greater than the string length. Therefore we are justified in using supergravity. Kaluza-Klein reduction of type IIB supergravity to six dimensions leads to six dimensional $(2,2)$ supergravity.

\footnotetext{
${ }^{11}$ The pure anti-de Sitter supergravity based on the super group $S U(1,1 \mid 2) \times S U(1,1 \mid 2)$ was constructed in 136 using the fact that it is a Chern-Simons theory.
} 
We show that the Kaluza-Klein spectrum of the six dimensional theory on $A d S_{3} \times$ $S^{3}$ can be completely organized as short multiplets of the supergroup $S U(1,1 \mid 2) \times$ $S U(1,1 \mid 2)$. We will follow the method developed by [43.

The massless spectrum of $(2,2)$ six-dimensional supergravity consists of: a graviton, 8 gravitinos, 5 two-forms, 16 gauge fields, 40 fermions and 25 scalars. Since these are massless, the physical degrees of freedom fall into various representations $R_{4}$ of the little group $S O(4)_{L}$ of $R^{(5,1)}$. For example, the graviton transforms as a $(\mathbf{3}, \mathbf{3})$ under the little group $S O(4)_{L}=S U(2)_{L} \times S \widetilde{S U}(2)_{L}$. On further compactifying $R^{(5,1)}$ into $A d S_{3} \times S^{3}$, each representation $R_{4}$ decomposes into various representations $R_{3}$ of $S O(3)$, the local Lorentz group of the $S^{3}$. This $S O(3) \simeq S U(2)$ is the diagonal $S U(2)$ of $S U(2)_{L} \times S \widetilde{U(2)_{L}}$. For example, the graviton decomposes as $\mathbf{1}+\mathbf{3}+\mathbf{5}$ under the $S O(3)$, the local Lorentz group of $S^{3}$. The dependence of each of these fields on the angles of $S^{3}$ leads to decomposition in terms of Kaluza-Klein modes on the $S^{3}$ which transforms according to some representation of the isometry group $S O(4)$ of $S^{3}$. Only those representations of $S O(4)$ occur in these decompositions which contain the representation $R_{3}$ of $S^{3}$. To be more explicit, consider the field $\phi_{R_{S O(3)}}\left(x_{0}, x_{5}, r, \theta, \phi, \chi\right)$ which transforms as some representation $R_{S O(3)}$ of the local Lorentz group of $S^{3}$. The Kaluza-Klein expansion of this field on $S^{3}$ is given by

$$
\phi_{R_{S O(3)}}\left(x_{0}, x_{5}, r, \theta, \phi, \chi\right)=\sum_{R_{S O(4)}} \tilde{\phi}_{R_{S O(4)}}\left(x_{0}, x_{5}, r\right) Y_{R_{S O(3)}}^{R_{S O(4)}}(\theta, \phi, \chi) .
$$

Here $Y_{R_{S O(3)}}^{R_{S O(4)}}(\theta, \phi, \chi)$ stands for the spherical harmonics on $S^{3}$. In the above expansion the only representation of $R_{S O(4)}$ allowed are the ones which contain $R_{S O(3)}$. For example, $\phi\left(x_{0}, x_{5}, r, \theta, \phi, \chi\right)$ which is a scalar under the local Lorentz group of $S^{3}$ can be expanded as

$$
\phi\left(x_{0}, x_{5}, r, \theta, \phi, \chi\right)=\sum_{\mathbf{m}, \mathbf{m}^{\prime} ; \mathbf{m}=\mathbf{m}^{\prime}} \tilde{\phi}_{\mathbf{m m}^{\prime}}\left(x_{0}, x_{5}, r\right) Y^{\left(\mathbf{m}, \mathbf{m}^{\prime}\right)}(\theta, \phi, \chi)
$$

Once the complete set of Kaluza-Klein modes are obtained we will organize them into short multiplets of the supergroup $S U(1,1 \mid 2) \times S U(1,1 \mid 2)$.

Let us now consider all the massless field of $(2,2)$ supergravity in six-dimensions individually. The graviton transforms as $(\mathbf{3}, \mathbf{3})$ of the little group in 6 dimensions. The Kaluza-Klein harmonics of this field according to the rules discussed above are

$$
\begin{gathered}
(\mathbf{1}, \mathbf{1})+2(\mathbf{2}, \mathbf{2})+(\mathbf{3}, \mathbf{1})+(\mathbf{1}, \mathbf{3}) \\
+3 \oplus_{\mathbf{m} \geq \mathbf{3}}(\mathbf{m}, \mathbf{m})+2 \oplus_{\mathbf{m} \geq \mathbf{2}}[(\mathbf{m}+\mathbf{2}, \mathbf{m})+(\mathbf{m}, \mathbf{m}+\mathbf{2})] \\
+\oplus_{\mathbf{m} \geq \mathbf{1}}[(\mathbf{m}+\mathbf{4}, \mathbf{m})+(\mathbf{m}, \mathbf{m}+\mathbf{4})]
\end{gathered}
$$

The little group representations of the 8 gravitinos is $4(\mathbf{2}, \mathbf{3})+4(\mathbf{3}, \mathbf{2})$. Their Kaluza-Klein harmonics are

$$
8[(\mathbf{1}, \mathbf{2})+(\mathbf{2}, \mathbf{1})]+16 \oplus_{\mathbf{m} \geq \mathbf{2}}[(\mathbf{m}+\mathbf{1}, \mathbf{m})+(\mathbf{m}, \mathbf{m}+\mathbf{1})]
$$




$$
+8 \oplus_{\mathbf{m} \geq \mathbf{1}}[(\mathbf{m}+\mathbf{3}, \mathbf{m})+(\mathbf{m}, \mathbf{m}+\mathbf{3})]
$$

The Kaluza-Klein harmonics of the 5 two-forms transforming in $(\mathbf{1}, \mathbf{3})+(\mathbf{3}, \mathbf{1})$ of the little group are

$$
10 \oplus_{\mathbf{m} \geq \mathbf{2}}(\mathbf{m}, \mathbf{m})+10 \oplus_{\mathbf{m} \geq \mathbf{1}}[(\mathbf{m}+\mathbf{2}, \mathbf{m})+(\mathbf{m}, \mathbf{m}+\mathbf{2})]
$$

The Kaluza-Klein harmonics of the 16 gauge fields, $(\mathbf{2}, \mathbf{2})$ are

$$
16(\mathbf{1}, \mathbf{1})+32 \oplus_{\mathbf{m} \geq \mathbf{2}}(\mathbf{m}, \mathbf{m})+16 \oplus_{\mathbf{m} \geq \mathbf{1}}[(\mathbf{m}, \mathbf{m}+\mathbf{2})+(\mathbf{m}+\mathbf{2}, \mathbf{m})]
$$

The 40 fermions $20(\mathbf{2}, \mathbf{1})+20(\mathbf{1}, \mathbf{2})$ give rise to the following harmonics

$$
40 \oplus_{\mathbf{m} \geq \mathbf{1}}[(\mathbf{m}, \mathbf{m}+\mathbf{1})+(\mathbf{m}+\mathbf{1}, \mathbf{m})]
$$

The 25 scalars $(\mathbf{1}, \mathbf{1})$ give rise to the harmonics

$$
25 \oplus_{\mathbf{m} \geq \mathbf{1}}(\mathbf{m}, \mathbf{m})
$$

Putting all this together the complete Kaluza-Klein spectrum of type IIB on $A d S_{3} \times$ $S^{3} \times T^{4}$ yields

$$
\begin{gathered}
42(\mathbf{1}, \mathbf{1})+69(\mathbf{2}, \mathbf{2})+48[(\mathbf{1}, \mathbf{2})+(\mathbf{2}, \mathbf{1})]+27[(\mathbf{1}, \mathbf{3})+(\mathbf{3}, \mathbf{1})] \\
70 \oplus_{\mathbf{m} \geq \mathbf{3}}(\mathbf{m}, \mathbf{m})+56 \oplus_{\mathbf{m} \geq \mathbf{2}}[(\mathbf{m}, \mathbf{m}+\mathbf{1})+(\mathbf{m}+\mathbf{1}, \mathbf{m})] \\
+28 \oplus_{\mathbf{m} \geq \mathbf{2}}[(\mathbf{m}, \mathbf{m}+\mathbf{2})+(\mathbf{m}+\mathbf{2}, \mathbf{m})]+8 \oplus_{\mathbf{m} \geq \mathbf{1}}[(\mathbf{m}, \mathbf{m}+\mathbf{3})+(\mathbf{m}+\mathbf{3}, \mathbf{m})] \\
+\oplus_{\mathbf{m} \geq \mathbf{1}}[(\mathbf{m}, \mathbf{m}+\mathbf{4})+(\mathbf{m}+\mathbf{4}, \mathbf{m})]
\end{gathered}
$$

We now organize the above Kaluza-Klein modes into short representations of $S U(1,1 \mid 2) \times S U(1,1 \mid 2)$ 43. The short multiplet of $S U(1,1 \mid 2)$ consists of the following states

\begin{tabular}{ccc}
$j$ & $L_{0}$ & Degeneracy \\
\hline$h$ & $h$ & $2 h+1$ \\
$h-1 / 2$ & $h+1 / 2$ & $2(2 h)$ \\
$h-1$ & $h+1$ & $2 h-1$
\end{tabular}

In the above table $j$ labels the representation of $S U(2)$ which is identified as one of the $S U(2)$ 's of the isometry group of $S^{3}$. $L_{0}$ denotes the conformal weight of the state. We denote the short multiplet of $S U(1,1 \mid 2) \times S U(1,1 \mid 2)$ as $\left(\mathbf{2 h}+\mathbf{1}, \mathbf{2} \mathbf{h}^{\prime}+\mathbf{1}\right)_{S}$. On organizing the Kaluza-Klein spectrum into short multiplets we get the following set

$$
\begin{gathered}
5(\mathbf{2}, \mathbf{2})_{S}+6 \oplus_{\mathbf{m} \geq \mathbf{3}}(\mathbf{m}, \mathbf{m})_{S} \\
\bigoplus_{\mathbf{m} \geq \mathbf{2}}\left[(\mathbf{m}, \mathbf{m}+\mathbf{2})_{S}+(\mathbf{m}+\mathbf{2}, \mathbf{m})_{S}+4(\mathbf{m}, \mathbf{m}+\mathbf{1})_{S}+4(\mathbf{m}+\mathbf{1}, \mathbf{m})_{S}\right]
\end{gathered}
$$

Equation (6.10) shows that there are $42(\mathbf{1}, \mathbf{1}) S O(4)$ representations in the supergravity Kaluza Klein spectrum. We know that one of these arises from the $s$-wave 
of $g_{55}$ from equation (6.4). This is one of the fixed scalars. 16(1, 1) comes from the $s$-waves of the 16 gauge fields (the components along $x^{5}$ ) as seen in equation (6.7). The remaining 25 comes from the 25 scalars of the six dimensional theory. We would like to see where these $42(\mathbf{1}, \mathbf{1})$ fit in the short multiplets of $S U(1,1 \mid 2) \times S U(1,1 \mid 2)$. From equation (6.12) one can read that 20 of them are in the $5(\mathbf{2}, \mathbf{2})_{S}$ with $\left(j=0, L_{0}=1 ; j=0, L_{0}=1\right) .6$ of them are in in $6(\mathbf{3}, \mathbf{3})_{S}$ with $\left(j=0, L_{0}=2 ; j=0, L_{0}=2\right)$. These correspond to the fixed scalars. Finally, the remaining 16 of them belong to $4(\mathbf{2}, \mathbf{3})_{S}+4(\mathbf{3}, \mathbf{2})_{S}$. 8 of them have $\left(j=0, L_{0}=1 ; j=0, L_{0}=2\right)$ and 8 of them have $\left(j=0, L_{0}=2 ; j=0, L_{0}=1\right)$. These scalars can be recognized as the intermediate scalars.

\section{Comparison of supergravity short multiplets with SCFT}

In Section 5.8 we have listed the complete set of short multiplets corresponding to single particle states of the $\mathcal{N}=(4,4) \mathrm{SCFT}$ on the orbifold $\mathcal{M}$. Comparing equation (5.55) and the list of short multiplets of single particle states obtained from supergravity in (6.12) we find that they are identical except for the presence of the following additional short multiplets in the SCFT

$$
2(\mathbf{1}, \mathbf{2})_{S}+2(\mathbf{2}, \mathbf{1})_{S}+(\mathbf{1}, \mathbf{3})_{S}+(\mathbf{3}, \mathbf{1})_{S}
$$

These correspond to non-propagating degrees of freedom in the supergravity [43]. Therefore they are not present in the list of short multiplets obtained form super-

gravity (6.12). Furthermore, note that in (5.55) we have ignored the contribution of short multiplets from the free $T^{4}$ which forms the part of the Higgs branch of the D1-D5 system. Thus the short multiplets in supergravity also ignores the contribution from the free torus.

It is pertinent here to mention that the AdS/CFT duality for D1-D5 systems in theories with 16 supercharges were studied in [137, 138. These theories were obtained by considering various orbifolds of type IIB.

\subsection{The supergravity moduli}

In this section we will analyze in detail the massless scalars in the near horizon geometry of the D1-D5 system. Type IIB supergravity compactified on $T^{4}$ has 25 scalars. There are 10 scalars $h_{i j}$ which arise from compactification of the metric. $i, j, k \ldots$ stands for the directions of $T^{4}$. There are 6 scalars $b_{i j}$ which arise from the Neveu-Schwarz $B$-field and similarly there are 6 scalars $b_{i j}^{\prime}$ from the RamondRamond $B^{\prime}$-field. The remaining 3 scalars are the ten-dimensional dilaton $\phi_{10}$, the Ramond-Ramond scalar $\chi$ and the Ramond-Ramond 4-form $C_{6789}$. These scalars parameterize the coset $S O(5,5) /(S O(5) \times S O(5))$. The near horizon limit of the D1-D5 system is $A d S_{3} \times S^{3} \times T^{4}$ (2.70). In this geometry 5 of the 25 scalars become massive [90]. They are the $h_{i i}$ (the trace of the metric of $T^{4}$ which is proportional 
to the volume of $T^{4}$ ), the 3 components of the anti-self dual part of the NeveuSchwarz $B$-field $b_{i j}^{-}$and a linear combination of the Ramond-Ramond scalar and the 4 -form 114. The massless scalars in the near horizon geometry parameterize the coset $S O(5,4) /(S O(5) \times S O(4))$ 139.

As we have seen the near horizon symmetries form the supergroup $S U(1,1 \mid 2) \times$ $S U(1,1 \mid 2)$. We have classified all the massless supergravity fields of type IIB supergravity on $A d S_{3} \times S^{3} \times T^{4}$ ignoring the Kaluza-Klein modes on $T^{4}$ according to the short multiplets of the supergroup $S U(1,1 \mid 2) \times S U(1,1 \mid 2)$. The isometries of the anti-de Sitter space allow us to relate the quantum number $L_{0}+\bar{L}_{0}$ to the mass of the scalar field through the relation 90 .

$$
h+\bar{h}=1+\sqrt{1+m^{2}}
$$

Here $m$ is the mass of the scalar in units of the radius of $A d S_{3}$ and $(h, \bar{h})$ is the eigenvalue of $L_{0}, \bar{L}_{0}$ under the classification of the scalar in short multiplets of $S U(1,1 \mid 2) \times S U(1,1 \mid 2)$. Thus the massless fields of the near horizon geometry of the D1-D5 system fall into the top component of the $5(\mathbf{2}, \mathbf{2})_{\mathrm{S}}$ short multiplet. We further classify these fields according to the representations of the $S O(4)_{I}$, the rotations of the $x_{6}, x_{7}, x_{8}, x_{9}$ directions. As we have mentioned before this is not a symmetry of the supergravity as it is compactified on $T^{4}$, but it can be used to classify states. The quantum number of the massless supergravity fields are listed below.

$\begin{array}{lccc}\text { Field } & \left.S U(2)_{I} \times S \widetilde{S(2}\right)_{I} & S U(2)_{E} \times S \widetilde{S(2)_{E}} & \text { Mass } \\ h_{i j}-\frac{1}{4} \delta_{i j} h_{k k} & (\mathbf{3}, \mathbf{3}) & (\mathbf{1}, \mathbf{1}) & 0 \\ b_{i j}^{\prime} & (\mathbf{3}, \mathbf{1})+(\mathbf{1}, \mathbf{3}) & (\mathbf{1}, \mathbf{1}) & 0 \\ \phi_{6} & (\mathbf{1}, \mathbf{1}) & (\mathbf{1}, \mathbf{1}) & 0 \\ a_{1} \chi+a_{2} C_{6789} & (\mathbf{1}, \mathbf{1}) & (\mathbf{1}, \mathbf{1}) & 0 \\ b_{i j}^{+} & (\mathbf{1}, \mathbf{3}) & (\mathbf{1}, \mathbf{1}) & 0\end{array}$

The linear combination appearing on the fourth line is the one that remains massless in the near-horizon limit. $\phi_{6}$ refers to the six-dimensional dilaton. The $S U(2)_{E} \times \widetilde{S U(2)_{E}}$ stands for the $S O(4)$ isometries of the $S^{3}$. All the above fields are s-waves of scalars in the near horizon geometry.

\section{3 $\mathrm{AdS}_{3} / \mathrm{CFT}_{2}$ correspondence}

We have already seen in Section 6.3 that all the supergravity modes can be organized as short multiplets of the SCFT on $\mathcal{M}$. This is evidence for Maldacena's AdS/CFT correspondence. Maldacena's conjecture [42, 62, 63, 90 for the case of the D1-D5 system states that string theory on $A d S_{3} \times S^{3} \times T^{4}$ is dual to the $1+1$ dimensional conformal field theory of the Higgs branch of gauge theory of the D1-D5 system. Here we briefly review the evidence for this conjecture from 
symmetries. To describe the D1-D5 system at a generic point in the moduli space we can use the $\mathcal{N}=(4,4) \mathrm{SCFT}$ on the orbifold $\mathcal{M} \times T^{4}$ to describe the Higgs branch of the gauge theory of the D1-D5 system as we have argued in Section 4. Here the dynamics on $T^{4}$ is decoupled from the symmetric product. However in string theory on $A d S_{3} \times S^{3} \times T^{4}$, all fields couple to gravity and no field is free. Thus in this case of the $A d S / C F T$ correspondence we have to ignore the free torus $T^{4}$. This is the same reason that the $U(1)$ of the $\mathcal{N}=4$ super Yang-Mills theory with gauge group $U(N)$ is ignored in the correspondence with string theory on $A d S_{5} \times S^{4}$. The gauge group used in the correspondence is in fact $S U(N)$ [140]. The volume of $T^{4}$ is of the order of string length and radius of $S^{3}$ is large, therefore we can pass over from string theory on $A d S_{3} \times S^{3} \times T^{4}$ to six-dimensional $(2,2)$ supergravity on $A d S_{3} \times S^{3}$. We will compare symmetries in the supergravity limit. The identification of the isometries of the near horizon geometry with that of the symmetries of the SCFT are given in the following table.

\begin{tabular}{|c|c|}
\hline Symmetries of the Bulk & Symmetries of SCFT \\
\hline (a) Isometries of $A d S_{3}$ & The global part of the Virasoro group \\
\hline$S O(2,2) \simeq S L(2, R) \times S \widetilde{(2, R)}$ & $S L(2, R) \times S \widetilde{(2, R)}$ \\
\hline (b) Isometries of $S^{3}$ & R-symmetry of the SCFT \\
\hline$S O(4)_{E} \simeq S U(2) \times S U(2)$ & $S U(2)_{R} \times \widetilde{S U(2)_{R}}$ \\
\hline (c) Sixteen near horizon supersymmetries & Global supercharges of $\mathcal{N}=(4,4) \mathrm{SCFT}$ \\
\hline (d) $S O(4)_{I}$ of $T^{4}$ & $S O(4)_{I}$ of $\tilde{T}^{4}$ \\
\hline
\end{tabular}

To summarize the $S U(1,1 \mid 2) \times S U(1,1 \mid 2)$ symmetry of the near horizon geometry is identified with the global part of the $\mathcal{N}=(4,4)$ SCFT on the orbifold $\mathcal{M}$ together with the identification of the $S O(4)_{I}$ algebra of $T^{4}$ and $\tilde{T}^{4}$.

\subsection{Supergravity moduli and the marginal operators}

We would like to match the twenty supergravity moduli appearing in (6.15) with the twenty marginal operators appearing in (5.19) and (5.29) by comparing their symmetry properties under the AdS/CFT correspondence 141.

The symmetries, or equivalently quantum numbers, to be compared under the AdS/CFT correspondence are as follows:

(a) The isometries of the supergravity are identified with the global symmetries of the superconformal field theory. For the $A d S_{3}$ case the symmetries form the supergroup $S U(1,1 \mid 2) \times S U(1,1 \mid 2)$. The identification of this supergroup with the global part of the $\mathcal{N}=(4,4)$ superalgebra leads to the mass-dimension relation (6.14). Since in our case the SCFT operators are marginal and the supergravity fields are massless, the mass-dimension relation is obviously satisfied.

(b) The $S U(2)_{E} \times S \widetilde{U U(2)_{E}}$ quantum number of the bulk supergravity field corresponds to the $S U(2)_{R} \times S \widetilde{S(2)_{R}}$ quantum number of the boundary operator. 
By an inspection of column three of the tables in (5.19), (5.29) and $(6.15)$, we see that these quantum numbers also match.

(c) The location of the bulk fields and the boundary operators as components of the short multiplet can be found by the supersymmetry properties of the bulk fields and the boundary operators. Noting the fact that all the twenty bulk fields as well as all the marginal operators mentioned above correspond to top components of short multiplets, this property also matches.

(d) The above symmetries alone do not distinguish between the twenty operators or the twenty bulk fields. To further distinguish these operators and the fields we identify the $S O(4)_{I}$ symmetry of the directions $x_{6}, x_{7}, x_{8}, x_{9}$ with the $S O(4)_{I}$ of the SCFT. At the level of classification of states this identification is reasonable though these are not actual symmetries. Using the quantum numbers under this group we obtain the following matching of the boundary operators and the supergravity moduli.

$\begin{array}{llc}\text { Operator } & \text { Field } & S U(2)_{I} \times S \widetilde{S U}(2)_{I} \\ \partial x_{A}^{\{i}(z) \bar{\partial} x_{A}^{j\}}(\bar{z})-\frac{1}{4} \delta^{i j} \partial x_{A}^{k} \bar{\partial} x_{A}^{k} & h_{i j}-\frac{1}{4} \delta_{i j} h_{k k} & (\mathbf{3}, \mathbf{3}) \\ \partial x_{A}^{[i}(z) \bar{\partial} x_{A}^{j]}(\bar{z}) & b_{i j}^{\prime} & (\mathbf{3}, \mathbf{1})+(\mathbf{1}, \mathbf{3}) \\ \partial x_{A}^{i}(z) \bar{\partial} x_{A}^{i}(\bar{z}) & \phi & (\mathbf{1}, \mathbf{1}) \\ \mathcal{T}^{1} & b_{i j}^{+} & (\mathbf{1}, \mathbf{3}) \\ \mathcal{T}^{0} & a_{1} \chi+a_{2} C_{6789} & \mathbf{( 1 , 1 )}\end{array}$

Note that both the representations $(\mathbf{1}, \mathbf{3})$ and $(\mathbf{1}, \mathbf{1})$ occur twice in the above table. This could give rise to a two-fold ambiguity in identifying either $(\mathbf{1}, \mathbf{3})$ or $(\mathbf{1}, \mathbf{1})$ operators with their corresponding bulk fields. The way we have resolved it here is as follows. The operators $\mathcal{T}^{1}$ and $\mathcal{T}^{0}$ correspond to blow up modes of the orbifold, and as we will show in Section 7 that these are related to the Fayet-Iliopoulos terms and the $\theta$-term in the D1-D5 gauge theory. Tuning these operators one can reach the singular SCFT [114 that corresponds to fragmentation of the D1-D5 system. In supergravity, similarly, it is only the moduli $b_{i j}^{+}$and $a_{1} \chi+a_{2} C_{6789}$ which affect the stability of the D1-D5 system [114, 142, 119]. As a result, it is $b_{i j}^{+}$(and not $b_{i j}^{\prime+}$ ) which should correspond to the operator $\mathcal{T}^{1}$ and similarly $a_{1} \chi+a_{2} C_{6789}$ should correspond to $\mathcal{T}^{0}$. Another reason for this identification is as follows. $b_{i j}^{+}$ and $a_{1} \chi+a_{2} C_{6789}$ are odd under world sheet parity while $b_{i j}^{\prime+}$ and $\phi$ are even under world sheet parity. In a $Z_{2}$ orbifolded theory there is a $Z_{2}$ symmetry which can be used to classify the states [143]. Under this symmetry the $Z_{2}$ quantum number of the twisted sectors is -1 and the $Z_{2}$ quantum number of the untwisted sectors is +1 . If under the $\mathrm{AdS} / \mathrm{CFT}$ correspondence one can identify these $Z_{2}$ quantum numbers in the boundary SCFT and the bulk then the correspondence we have made is further justified.

Thus, we arrive at a one-to-one correspondence between operators of the SCFT and the supergravity moduli. 


\section{Location of the symmetric product}

In the previous section we have studied the moduli of the D1-D5 system in detail. In (6.16) we have listed all the supergravity moduli and their corresponding operators in the SCFT on $\mathcal{M}$. The D1-D5 system is unspecified until all its moduli are given. In this section we will find the location of the free field orbifold $\mathcal{N}=(4,4)$ SCFT theory on $\mathcal{M}$ in the the D1-D5 moduli space.

It is easy to see from the mass formula of the D1-D5 system, that the D1D5 system is marginally stable to decay. The mass per unit length of the D1-D5 system is given by

$$
M=\frac{1}{g_{s} 2 \pi \alpha^{\prime}}\left(Q_{1}+v Q_{5}\right)
$$

Here $v$ is defined in (2.69). Note that the above formula is linear in $Q_{1}$ and $Q_{5}$, therefore it does not cost any energy for the D1-D5 system to decay to sub-systems with smaller values of $Q_{1}$ and $Q_{5}$. But, when any of the moduli in (6.16) is turned on then the D1-D5 system is stable. In fact there is a binding energy which prevents its decay. To see this let us turn on one of the moduli in (6.16). Consider turning on the self-dual Neveu-Schwarz $B$-field along the D5-brane direction. We can choose it to be given by

$$
B_{i j}=\frac{1}{2 \pi \alpha^{\prime}}\left(\begin{array}{cccc}
0 & b & 0 & 0 \\
-b & 0 & 0 & 0 \\
0 & 0 & 0 & b \\
0 & 0 & -b & 0
\end{array}\right)
$$

Here $i, j$ runs from $6, \ldots 9$. For convenience let the metric of on the $T^{4}$ be $\delta_{i j}$ and $v=1$. To demonstrate that there is a binding energy it is sufficient to consider the case of $Q_{1}=1$ and $Q_{5}=1$. The mass ${ }^{12}$ of a single D1 brane is given by

$$
M_{\mathrm{D} 1}=\frac{1}{g_{s} 2 \pi \alpha^{\prime}}
$$

Similarly the mass of the D5 brane wrapped on $T^{4}$ with the B-field (7.2) is given by

$$
M_{\mathrm{D} 5}=\frac{1}{g_{s} 2 \pi \alpha^{\prime}}\left(1+b^{2}\right)
$$

It is easy to understand this mass formula from the Dirac-Born-Infeld action

$$
S=\frac{1}{g_{s} 2 \pi \alpha^{\prime}} \sqrt{\operatorname{Det}(G+2 \pi B)}
$$

where $G$ is the induced metric. Substituting the value of $B$ from (7.2) and the metric of the $T^{4}$ and expanding the Dirac-Born Infeld action in the static gauge

\footnotetext{
${ }^{12}$ Here mass refers to mass per unit length
} 
we obtain the mass for the D5 brane with the B-field as given by (7.4). The mass of the D1-D5 system with the B-field is given by 446]

$$
M=\frac{1}{g_{s} 2 \pi \alpha^{\prime}} \sqrt{\left.\left(Q_{1}+\left(1-b^{2}\right) Q_{5}\right)^{2}+4 b^{2} Q_{5}^{2}\right)}
$$

Here $Q_{1}$ and $Q_{5}$ stand for the number of D1 and D5 branes respectively. Substituting $Q_{1}=Q_{5}=1$ we get

$$
M_{\mathrm{D} 1-\mathrm{D} 5}=\frac{1}{g_{s} 2 \pi \alpha^{\prime}} \sqrt{4+b^{4}}
$$

The the binding energy is given by

$$
\Delta M=\left(M_{\mathrm{D} 1}+M_{\mathrm{D} 5}\right)-M_{\mathrm{D} 1-\mathrm{D} 5}
$$

It is easy to see this binding energy is positive ${ }^{13}$. One can repeat similar calculations with the other moduli given in (6.16) and demonstrate the existence of a positive binding energy. This issue of the stability of the D1-D5 system with the various moduli turned on has been discussed in [114, 119, 142, 88, 144].

It has been observed in 114 that the effective theory of a single D1 brane separating off the D1-D5 bound state is a linear dilaton theory. This was derived by studying the dynamics of a D1 brane close to the boundary of $A d S_{3}$. In section 7.1 we derive the effective theory of a set of $q_{1}$ D1 branes and $q_{5}$ D 5 branes splitting of the D1-D5 bound state. The AdS/CFT correspondence suggests that this decay of the D1-D5 system should also be seen from the conformal field theory of the Higgs branch of the D1-D5 system. It is convenient to extract the dynamics of the decay from the D1-D5 gauge theory. In fact such a decay signals a singularity in the world volume gauge theory associated with the origin of the Higgs branch. The dynamics of the decay can be extracted from the D1-D5 gauge theory using the methods developed by [145. In section 7.3 we extract the dynamics of the decay from the D1-D5 gauge theory and show that it is described by the same linear dilaton theory observed by [114 in supergravity.

The singularity mentioned above leads to a singular conformal field theory. However, generic values of the supergravity moduli which do not involve fragmentation into constituents are described by well-defined conformal field theories and therefore string perturbation theory makes sense. We have seen in section 5.6 that the important singularity structure of the $\mathcal{M}=(4,4) \mathrm{SCFT}$ on the orbifold $\mathcal{M}$ is locally of the type $R^{4} / Z_{2}$. The resolution of this singularity gives rise to marginal operators. An orbifold theory realized as a free field SCFT on $R^{4} / Z_{2}$ is nonsingular as all correlations functions are finite. The reason for this can be understood from

\footnotetext{
${ }^{13}$ If the NS B-field was anti-self dual then the binding energy is zero, and the D1-D5 system is marginally stable.
} 


\section{Linear sigma model (UV)}

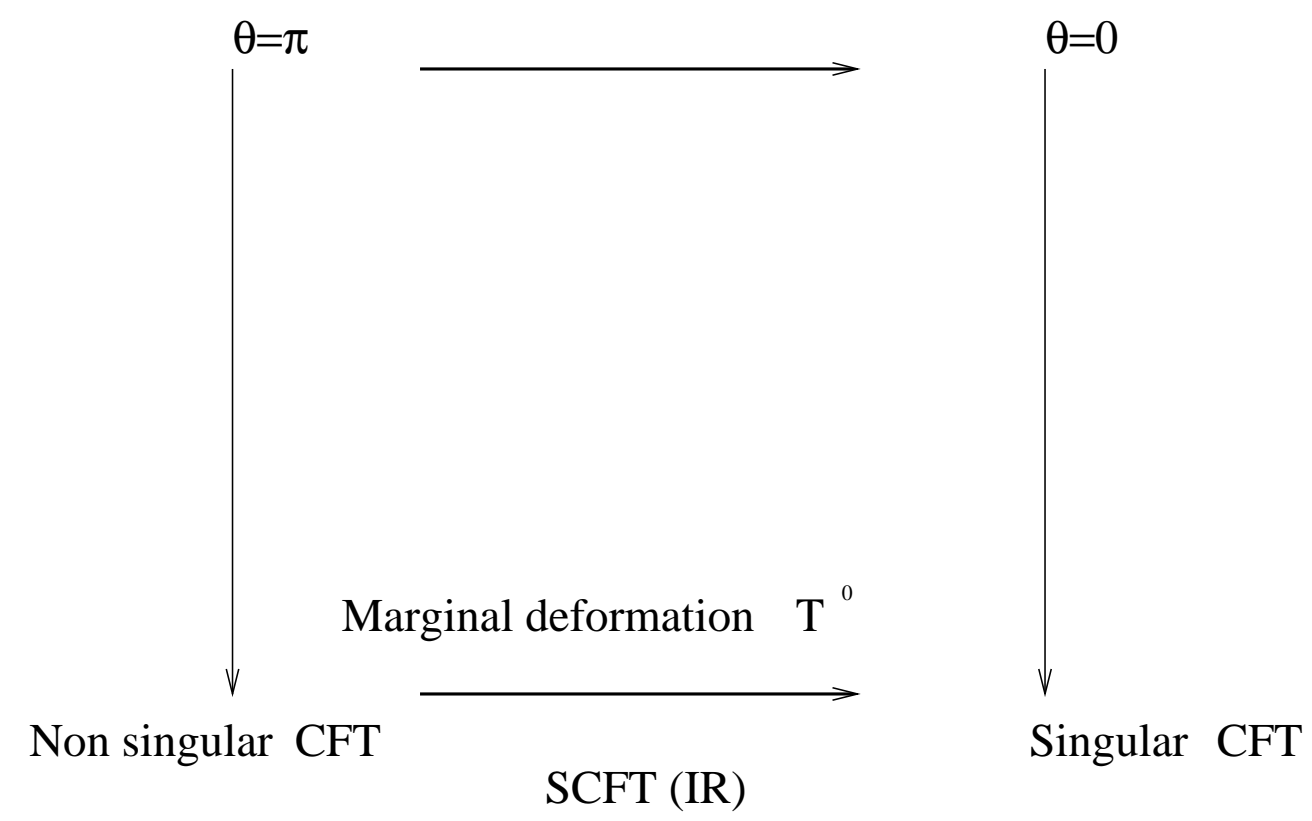

Figure 4: Linear sigma model and CFT description of the $R^{4} / Z_{2}$ singularity

the linear sigma model description of the $R^{4} / Z_{2}$ singularity which will be discussed in section 7.2. We will see that the though the $R^{4} / Z_{2}$ singularity is geometrically singular the SCFT is finite because it corresponds to a non-zero theta term in the linear sigma model. The geometric resolution of this singularity corresponds to adding Fayet-Iliopoulos terms to the D-term equations of the linear sigma model. This deforms the $R^{4} / Z_{2}$ singularity to an Eguchi-Hansen space. In the orbifold theory this deformation is caused by the twist operator $\mathcal{T}^{1}$. The Eguchi-Hansen space is asymptotically $R^{4} / Z_{2}$ but the singularity at the origin is blown up to a 2-sphere. One can use the $S U(2)_{R}$ symmetry of the linear sigma model to rotate the three Fayet-Iliopoulos terms to one term. This term corresponds to the radius of the blown up 2-sphere. The theta term of the linear sigma model corresponds to $B$-flux through the 2 -sphere. The change of this $B$-flux is caused by deforming the orbifold SCFT by the twist operator $\mathcal{T}^{0}$. Thus SCFT realized as a free field theory on the orbifold $R^{4} / Z_{2}$ is regular even though the 2 -sphere is squashed to zero size because of the non-zero value of $B$-flux trapped in the squashed 2 -sphere [146]. We summarize this discussion in (Fig ⿶).

For most of our discussion we have assumed that the Higgs branch of the D1D5 gauge theory is a resolution $\mathcal{N}=(4,4)$ theory on $\mathcal{M}$. Furthermore we have realized this theory as a free field theory with orbifold identification. This implies that we are at a point in the moduli space of the D1-D5 system at which the orbifold is geometrically singular but because of the non-zero value of the theta term the 
SCFT is regular and not at the singularity corresponding to fragmentation. In other words, the orbifold SCFT corresponds to a bound state of $Q_{1}$ D1 branes and $Q_{5}$ D 5 branes (henceforth denoted as the $\left(Q_{1}, Q_{5}\right)$ bound state). The supergravity solution (2.70) has no moduli turned on. This implies that the SCFT dual is singular and is far away in moduli space from the regular conformal theory on $\mathcal{M}$. But, the fact that we could show that the all the short multiplets of the supergravity modes on $A d S_{3} \times S^{3}$ is in one-to-one correspondence with the short multiplets of the SCFT on $\mathcal{M}$ implies that these multiplets are protected from non-renormalization theorems. This will be discussed in detail in section 9 .

\subsection{Dynamics of the decay of the D1-D5 system from gravity}

We consider a set of $\left(q_{1}, q_{5}\right)$ test D-branes with $q_{1}, q_{5} \ll Q_{1}, Q_{5}$ close to the boundary of $A d S_{3}$ but separated from the rest of the branes of the D1-D5 system. When the test branes are close to the boundary it is easy to see using the UV/IR correspondence that the the gauge group is broken to $U\left(q_{1}\right) \times U\left(q_{5}\right) \times U\left(Q_{1}-q_{1}\right) \times U(Q-$ $\left.q_{5}\right)$ from $U\left(Q_{1}\right) \times U\left(Q_{5}\right)$ in the IR. Thus we can extract the infrared dynamics of the decay of the D1-D5 system from gravity.

Lets us first consider the case when $q_{5}=0$ [114]. The AdS/CFT correspondence tells us that we need to consider $q_{1}$ D1 branes in the background of $A d S_{3} \times S^{3} \times T^{4}$. (We will work in the Euclidean $A d S_{3}$ coordinates.) The radius of $S^{3}$ and the anti-de Sitter space is given by $r_{0}=\sqrt{\alpha^{\prime}}\left(g_{6}^{2} Q_{1}^{\prime} Q_{5}^{\prime}\right)^{1 / 4} 14$, where $Q_{1}^{\prime}=Q_{1}-q_{1}$ and $Q_{5}^{\prime}=Q_{5}-q_{1}$. For the supergravity to be valid we need to consider the limit $r_{0} \gg 0$. Let us focus on the distance between the boundary of $A d S_{3} \times S^{3} \times T^{4}$ and the set of $q_{1} \mathrm{D} 1$ branes. We are interested in the infrared description of the splitting process. By the UV/IR correspondence the D1 branes should be close to the boundary of the $A d S_{3} \times S^{3} \times T^{4}$ to obtain the infrared description of the splitting process in the supergravity. We assume that the D1 branes are fixed at a particular point on the $S^{3}$ and the $T^{4}$. The action of $q_{1} \mathrm{D} 1$ branes in the background of $A d S_{3}$ and the Ramond-Ramond two-form $B_{05}$ is given by the DBI action. We can use the DBI action for multiple D1 branes as we are interested only in the dynamics of the centre of mass of thee collection of $q_{1} \mathrm{D} 1$ branes. The DBI action of $q_{1} \mathrm{D} 1$ branes is given by

$$
S=\frac{q_{1}}{2 \pi g_{s} \alpha^{\prime}} \int d^{2} \sigma e^{-\phi} \sqrt{\operatorname{det}\left(g_{\alpha \beta}^{\text {ind }}\right)}-\frac{q_{1}}{2 \pi g_{s} \alpha^{\prime}} \int B
$$

where $\sigma$ stands for the world volume coordinates and $\alpha, \beta$ label these coordinates. $g_{\alpha \beta}^{\text {ind }}$ is the induced metric on the world volume. $B$ is the Ramond-Ramond 2form potential. We chose a gauge in which the world volume coordinates are the coordinates of the boundary of the $A d S_{3}$. Let the metric on the boundary be

\footnotetext{
${ }^{14}$ We have called this quantity $R=\sqrt{\alpha^{\prime}} l$ in $(2.73),(11.4)$; the $r_{0}$ here is not be confused with the non-extremality parameter, such as in (2.39).
} 
$g_{\alpha \beta}(\sigma)$. One can extend the metric $g_{\alpha \beta}(\sigma)$ on the boundary to the interior of $A d S_{3}$ in the neighbourhood of the boundary [114]. This is given by

$$
d s^{2}=\frac{r_{0}^{2}}{t^{2}}\left(d t^{2}+\hat{g}_{\alpha \beta}(\sigma, t) d \sigma^{\alpha} d \sigma^{\beta}\right)
$$

with

$$
\hat{g}_{\alpha \beta}(\sigma, 0)=g_{\alpha \beta}(\sigma), \quad \hat{g}_{\alpha \beta}(\sigma, t)=g_{\alpha \beta}(\sigma)-t^{2} P_{\alpha \beta}+O\left(t^{3}\right)+\ldots
$$

Here $g_{\alpha \beta} P^{\alpha \beta}=R / 2 . \quad R$ is the world sheet curvature. The global coordinates of Euclidean $A d S_{3}$ is given by ${ }^{15}$

$$
d s^{2}=r_{0}^{2}\left(d \phi^{2}+\sinh ^{2} \phi d \Omega^{2}\right)
$$

where $d \Omega^{2}$ is the round metric on $S^{2}$. Near the boundary the metric is given by

$$
d s^{2}=r_{0}^{2}\left(d \phi^{2}+\frac{e^{2 \phi}}{4} d \Omega^{2}\right)
$$

Motivated by this we use $\phi$ defined as $t=2 e^{-\phi}$ to measure the distance from the boundary of $A d S_{3}$. Substituting the metric in (7.10) and the near horizon value of the Ramond-Ramond 2-form and the dilaton in (7.9) we obtain the following effective action of the D1 branes near the boundary.

$$
\begin{aligned}
S & =\frac{q_{1} r_{0}^{2}}{4 \pi g_{s} \alpha^{\prime}} \sqrt{\frac{Q_{5}^{\prime} v}{Q_{1}^{\prime}}} \int \sqrt{g}\left(\partial_{\alpha} \phi \partial^{\alpha} \phi+\phi R-\frac{1}{2} R+O\left(e^{-2 \phi}\right)\right) \\
& =\frac{q_{1} Q_{5}}{4 \pi} \int \sqrt{g}\left(\partial_{\alpha} \phi \partial^{\alpha} \phi+\phi R-\frac{1}{2} R+O\left(e^{-2 \phi}\right)\right)
\end{aligned}
$$

Now consider the case when $q_{1}=0$. The $q_{5}$ D 5 branes are wrapped on $T^{4}$. Therefore the world volume of the D5 branes is of the form $M_{2} \times T^{4}$ where $M_{2}$ is any 2-manifold. The D5 branes are located at a point on the $S^{3}$. We ignore the fluctuations on $T^{4}$ as we are interested in the dynamics on $A d S_{3}$. The DBI action of $q_{5} \mathrm{D} 5$ branes is given by

$$
\frac{q_{5}}{32 \pi^{5} g_{s} \alpha^{\prime 3}}\left(\int d^{6} \sigma e^{-\phi} \sqrt{\operatorname{det}\left(g_{\alpha \beta}^{\text {ind }}\right)}-\int C^{6}\right)
$$

where $C^{6}$ is the Ramond-Ramond 6-form potential coupling to the D5 brane. Performing a similar calculation for the D5 branes and substituting the near horizon values of the 6-from Ramond-Ramond potential, the dilaton and the volume of $T^{4}$ one obtains the following effective actions for the D5 branes

$$
\begin{aligned}
S & =\frac{q_{5} r_{0}^{2}}{4 \pi g_{2} \alpha^{\prime}} \sqrt{\frac{Q_{1}^{\prime} v}{Q_{5}^{\prime}}} \int \sqrt{g}\left(\partial_{\alpha} \phi \partial^{\alpha} \phi+\phi R-\frac{1}{2} R+O\left(e^{-2 \phi}\right)\right) \\
& =\frac{q_{5} Q_{1}}{4 \pi} \int \sqrt{g}\left(\partial_{\alpha} \phi \partial^{\alpha} \phi+\phi R-\frac{1}{2} R+O\left(e^{-2 \phi}\right)\right)
\end{aligned}
$$

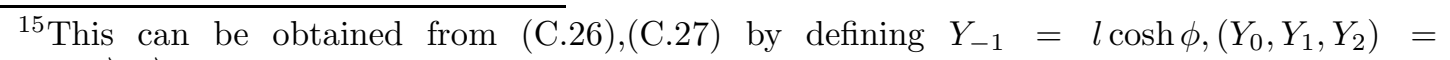
$l \sinh \phi \vec{\Omega}, \vec{\Omega} \in S^{2}$, and then replacing the notation $l$ by $r_{0}$, see the previous footnote (14).
} 
For the case when $q_{1} \neq 0$ and $q_{5} \neq 0$ and we just add the contribution from (7.14) and (7.16) to obtain the effective action of the $\left(q_{1}, q_{5}\right)$ string in $A d S_{3}$. The reason we can do this is because there is no force between the test D1 and D5 branes. Thus to the leading order in $\phi$ the total effective action of the $\left(q_{1}, q_{5}\right)$ string near the boundary is given by

$$
S=\frac{\left(q_{1} Q_{5}+q_{5} Q_{1}^{\prime}\right)}{4 \pi} \int \sqrt{g}\left(\partial_{\alpha} \phi \partial^{\alpha} \phi+\phi R-\frac{1}{2} R\right)
$$

Rescaling $\phi$ so that the the normalization of the kinetic energy term is canonical one obtains a linear dilaton action with a back ground charge given by

$$
Q_{\mathrm{SUGRA}}=\sqrt{2\left(q_{1} Q_{5}^{\prime}+q_{5} Q_{1}^{\prime}\right)}
$$

To summarize, the effective dynamics of the $\left(Q_{1}, Q_{5}\right)$ D1-D5 system by decaying into $\left(q_{1}, q_{5}\right)$ branes is governed by linear dilaton theory with background charge given by (7.18). Note that the linear dilaton theory in (7.17) is strongly coupled at $\phi \rightarrow \infty$, the boundary of $A d S_{3}$.

If a similar analysis is performed for the supergravity solution with the self dual NS $B$-field turned on (2.48), one obtains a potential for the linear dilaton which prevents the coupling to grow to infinity at the boundary of $A d S_{3}$ [88]. Thus the effective theory is non-singular in the presence of the NS $B$-field.

\subsection{The linear sigma model description of $R^{4} / Z_{2}$}

The linear sigma model is a $1+1$ dimensional $U(1)$ gauge theory with $(4,4)$ supersymmetry [147]. It has 2 hypermultiplets charged under the $U(1)$. The scalar fields of the hypermultiplets can be organized as doublets under the $S U(2)_{R}$ symmetry of the $(4,4)$ theory as

$$
\chi_{1}=\left(\begin{array}{c}
A_{1} \\
B_{1}^{\dagger}
\end{array}\right) \text { and } \chi_{2}=\left(\begin{array}{c}
A_{2} \\
B_{2}^{\dagger}
\end{array}\right)
$$

The $A$ 's have charge +1 and the $B$ 's have charge -1 under the $U(1)$. The vector multiplet has 4 real scalars $\varphi_{i}, i=1, \ldots, 4$. They do not transform under the $S U(2)_{R}$. One can include 4 parameters in this theory consistent with $(4,4)$ supersymmetry. They are the 3 Fayet-Iliopoulos terms and the theta term.

Let us first investigate the hypermultiplet moduli space of this theory with the 3 Fayet-Iliopoulos terms and the theta term set to zero. The Higgs phase of this theory is obtained by setting $\phi_{i}$ and the D-terms to zero. The D-term equations are

$$
\begin{aligned}
\left|A_{1}\right|^{2}+\left|A_{2}\right|^{2}-\left|B_{1}\right|^{2}-\left|B_{2}\right|^{2} & =0 \\
A_{1} B_{1}+A_{2} B_{2} & =0
\end{aligned}
$$


The hypermultiplet moduli space is the space of solutions of the above equations modded out by the $U(1)$ gauge symmetry. Counting the number of degrees of freedom indicate that this space is 4 dimensional. To obtain the explicit form of this space it is convenient to introduce the following gauge invariant variables

$$
\begin{gathered}
M=A_{1} B_{2} \quad N=A_{2} B_{1} \\
P=A_{1} B_{1}=-A_{2} B_{2}
\end{gathered}
$$

These variables are not independent. Setting the D-terms equal to zero and modding out the resulting space by $U(1)$ is equivalent to the equation

$$
P^{2}+M N=0 .
$$

This homogeneous equation is an equation of the space $R^{4} / Z_{2}$. To see this the solution of the above equation can be parameterized by 2 complex numbers $(\zeta, \eta)$ such that

$$
P=i \zeta \eta \quad M=\zeta^{2} \quad N=\eta^{2}
$$

Thus the point $(\zeta, \eta)$ and $(-\zeta,-\eta)$ are the same point in the space of solutions of (7.23). We have shown that the hypermultiplet moduli space is $R^{4} / Z_{2}$.

The above singularity at the origin of the moduli space is a geometric singularity in the hypermultiplet moduli space. We now argue that this singularity is a genuine singularity of the SCFT that the linear sigma model flows to in the infrared. At the origin of the classical moduli space the Coulomb branch meets the Higgs branch. In addition to the potential due to the D-terms the linear sigma model contains the following term in the superpotential ${ }^{16}$

$$
V=\left(\left|A_{1}\right|^{2}+\left|A_{2}\right|^{2}+\left|B_{1}\right|^{2}+\left|B_{2}\right|^{2}\right)\left(\varphi_{1}^{2}+\varphi_{2}^{2}+\varphi_{3}^{2}+\varphi_{4}^{2}\right)
$$

Thus at the origin of the hypermultiplet moduli space a flat direction for the Coulomb branch opens up. The ground state at this point is not normalizable due to the non-compactness of the Coulomb branch. This renders the infrared SCFT singular.

This singularity can be avoided in two distinct ways. If one turns on the Fayet-Iliopoulos D-terms, the D-term equations are modified to [147] ${ }^{17}$

$$
\begin{aligned}
\left|A_{1}\right|^{2}+\left|A_{2}\right|^{2}-\left|B_{1}\right|^{2}-\left|B_{2}\right|^{2} & =r_{3} \\
A_{1} B_{1}+A_{2} B_{2} & =r_{1}+i r_{2}
\end{aligned}
$$

\footnotetext{
${ }^{16}$ These terms can be understood from the coupling $A_{\mu} A^{\mu} \chi^{*} \chi$ in six dimensions, and recognizing that under dimensional reduction to two dimensions $\varphi_{i}$ 's appear from the components of $A_{\mu}$ in the compact directions.

${ }^{17}$ cf. Eqns. (4.14), 4.15), whose parameters $\zeta, \zeta_{c}$ are related to the present parameters $r_{1}, r_{2}, r_{3}$ by $r_{3} \equiv \zeta, r_{1}+i r_{2} \equiv \zeta_{c}$.
} 
Where $r_{1}, r_{2}, r_{3}$ are the 3 Fayet-Iliopoulos D-terms transforming as the adjoint of the $S U(2)_{R}$. Now the origin is no more a solution of these equations and the non-compactness of the Coulomb branch is avoided. In this case wave-functions will have compact support on the Coulomb branch. This ensures that the infrared SCFT is non-singular. Turning on the Fayet-Iliopoulos D-terms thus correspond to the geometric resolution of the singularity. The resolved space is known to be [147, 146] described by an Eguchi-Hanson metric in which $r_{1,2,3}$ parameterize a shrinking two-cycle.

The second way to avoid the singularity in the SCFT is to turn on the theta angle $\theta$. This induces a constant electric field in the vacuum. This electric field is screened at any other point than the origin in the hypermultiplet moduli space as the $U(1)$ gauge field is massive with a mass proportional to the vacuum expectation value of the hypers. At the origin the $U(1)$ field is not screened and thus it contributes to the energy density of the vacuum. This energy is proportional to $\theta^{2}$. Thus turning on the theta term lifts the flat directions of the Coulomb branch. This ensures that the corresponding infrared SCFT is well defined though the hypermultiplet moduli space remains geometrically singular. In terms of the Eguchi-Hanson space, the $\theta$-term corresponds to a flux of the antisymmetric tensor through the two-cycle mentioned above.

The $(4,4)$ SCFT on $R^{4} / Z_{2}$ at the orbifold point is well defined. Since the orbifold has a geometric singularity but the SCFT is non-singular it must correspond to the linear sigma model with a finite value of $\theta$ and the Fayet-Iliopoulos D-terms set to zero. Deformations of the $R^{4} / Z_{2}$ orbifold by its 4 blow up modes correspond to changes in the Fayet-Iliopoulos D-terms and theta term of the linear sigma model ${ }^{18}$ The global description of the moduli of a $\mathcal{N}=(4,4)$ SCFT on a resolved $R^{4} / Z_{2}$ orbifold is provided by the linear sigma model. In conclusion let us describe this linear sigma model in terms of the gauge theory of D-branes. The theory described above arises on a single D1-brane in presence of 2 D5-branes. The singularity at the point $r_{1}, r_{2}, r_{3}, \theta=0$ is due to noncompactness of the flat direction of the Coulomb branch. Thus it corresponds to the physical situation of the D1-brane leaving the D5-branes.

\subsection{The gauge theory relevant for the decay of the D1-D5 system}

As we have seen in Section 5.6 the resolutions of the $\mathcal{N}=(4,4) \operatorname{SCFT}$ on $\mathcal{M}$ is described by 4 marginal operators which were identified in the previous subsection with the Fayet-Iliopoulos D-terms and the theta term of the linear sigma model description of the $R^{4} / Z_{2}$ singularity. We want to now indicate how these four

\footnotetext{
${ }^{18}$ If we identify the $S U(2)_{R}$ of the linear sigma-model with $\left.\widetilde{S U(2)}\right)_{I}$ of the orbifold SCFT, then the Fayet-Iliopoulos parameters will correspond to $\mathcal{T}^{1}$ and the $\theta$-term to $\mathcal{T}^{0}$. This is consistent with Witten's observation 113 that $S O(4)_{E}$ symmetry of the linear sigma-model (one that rotates the $\phi_{i}$ 's) corresponds to the $S U(2)_{R}$ of the orbifold SCFT.
} 
parameters would make their appearance in the gauge theory description of the full D1-D5 system.

Motivated by the D-brane description of the $R^{4} / Z_{2}$ singularity we look for the degrees of freedom characterizing the break up of $\left(Q_{1}, Q_{5}\right)$ system to $\left(q_{1}, q_{5}\right)$ and $\left(Q_{1}^{\prime}, Q_{5}^{\prime}\right)$ where $Q_{1}^{\prime}=Q_{1}-q_{1}$ and $Q_{5}^{\prime}=Q_{5}-q_{5}$. Physically the relevant degree of freedom describing this process is the relative coordinate between the centre of mass of the $\left(q_{1}, q_{5}\right)$ system and the $\left(Q_{1}^{\prime}, Q_{5}^{\prime}\right)$. We will describe the effective theory of this degree of freedom below.

For the bound state $\left(Q_{1}, Q_{5}\right)$ the hypermultiplets, $\chi$ are charged under the relative $U(1)$ of $U\left(Q_{1}\right) \times U\left(Q_{5}\right)$, that is under the gauge field $A_{\mu}=\operatorname{Tr}_{U\left(Q_{1}\right)}\left(A_{\mu}\right)$ $\operatorname{Tr}_{U\left(Q_{5}\right)}\left(A_{\mu}\right)$. The relative $U(1)$ gauge multiplet corresponds to the degree of freedom of the relative coordinate between the centre of mass of the collection of $Q_{1}$ D1-branes and $Q_{5}$ D5-branes. At a generic point of the Higgs phase, all the $\chi$ 's have expectation values, thus making this degree of freedom becomes massive. This is consistent with the fact that we are looking at the bound state $\left(Q_{1}, Q_{5}\right)$.

Consider the break up of the $\left(Q_{1}, Q_{5}\right)$ bound state to the bound states $\left(q_{1}, q_{5}\right)$ and $\left(Q_{1}^{\prime}, Q_{5}^{\prime}\right)$. To find out the charges of the hypermultiplets under the various $U(1)$, we will organize the hypers as

$$
\left.\chi=\left(\begin{array}{cc}
\chi_{a \bar{b}} & \chi_{a^{\prime} \bar{b}^{\prime}} \\
\chi_{a^{\prime} \bar{b}} & \chi_{a^{\prime} \bar{b}^{\prime}}
\end{array}\right), \quad Y_{i}^{(1)}=\left(\begin{array}{cc}
Y_{i(a \bar{a})}^{(1)} & Y_{i\left(a \bar{a}^{\prime}\right)}^{(1)} \\
Y_{i\left(a^{\prime} \bar{a}\right)}^{(1)} & Y_{i\left(a^{\prime} \bar{a}^{\prime}\right)}^{(1)}
\end{array}\right) \quad \text { and } \quad Y_{i}^{(5)}=\left(\begin{array}{cc}
Y_{i(b \bar{b})}^{(5)} & Y_{i\left(b b^{\prime}\right)}^{(5)} \\
Y_{i\left(b^{\prime} \bar{b}\right)}^{(5)} & Y_{i\left(b^{\prime} \bar{b}^{\prime}\right)}^{(5)}
\end{array}\right) .27\right)
$$

where $a, \bar{a}$ runs from $1, \ldots, q_{1}, b, \bar{b}$ from $1, \ldots, q_{5}, a^{\prime} \bar{a}^{\prime}$ from $1, \ldots Q_{1}^{\prime}$ and $b^{\prime}, \bar{b}^{\prime}$ from $1 \ldots, Q_{5}^{\prime}$. We organize the scalars of the vector multiplet corresponding to the gauge group $U\left(Q_{1}\right)$ and $U\left(Q_{5}\right)$ as

$$
\phi_{m}^{(5)}=\left(\begin{array}{ll}
\phi_{m}^{(1) a \bar{a}} & \phi_{m}^{(1) a \bar{a}^{\prime}} \\
\phi_{m}^{(1) a a^{\prime}} & \phi_{m}^{(2) a^{\prime} \bar{a}^{\prime}}
\end{array}\right) \quad \text { and } \quad \phi_{m}^{(5)}=\left(\begin{array}{ll}
\phi_{m}^{(5) b \bar{b}} & \phi_{m}^{(5) b \bar{b}^{\prime}} \\
\phi_{m}^{(5) b^{\prime} \bar{b}} & \phi_{m}^{(5) b^{\prime} \bar{b}^{\prime}}
\end{array}\right)
$$

where $m=1,2,3,4$.

Let us call the the $U(1)$ gauge fields (traces) of $U\left(q_{1}\right), U\left(q_{5}\right), U\left(Q_{1}^{\prime}\right), U\left(Q_{5}^{\prime}\right)$ as $A_{1}, A_{5}, A_{1}^{\prime}, A_{5}^{\prime}$ respectively. We will also use the notation $A_{ \pm} \equiv A_{1} \pm A_{5}$ and $A_{ \pm}^{\prime} \equiv A_{1}^{\prime} \pm A_{5}^{\prime}$.

As we are interested in the bound states $\left(q_{1}, q_{5}\right)$ and $\left(Q_{1}^{\prime}, Q_{5}^{\prime}\right)$, in what follows we will work with a specific classical background in which we give vev's to the blockdiagonal hypers $\chi_{a b}, \chi_{a^{\prime} b^{\prime}}, Y_{i(a \bar{a})}^{(1)}, Y_{i(b \bar{b})}^{(5)}, Y_{i\left(a^{\prime} \bar{a}^{\prime}\right)}^{(1)}$ and $Y_{i\left(b^{\prime} \bar{b}^{\prime}\right)}^{(5)}$. These vev's are chosen so that the classical background satisfies the D-term equations (4.13).

The vev's of the $\chi$ 's render the fields $A_{-}$and $A_{-}^{\prime}$ massive with a mass proportional to vev's. In the low energy effective Lagrangian these gauge fields can therefore be neglected. In the following we will focus on the $U(1)$ gauge field $A_{r}=1 / 2\left(A_{+}-A_{+}^{\prime}\right)$ which does not get mass from the above vev's. The gauge multiplet corresponding to $A_{r}$ contains four real scalars denoted below by $\varphi_{m}$. 
These represent the relative coordinate between the centre of mass of the $\left(q_{1}, q_{5}\right)$ and the $\left(Q_{1}^{\prime}, Q_{5}^{\prime}\right)$ bound states. We will be interested in the question whether the $\varphi_{m}$ 's remain massless or otherwise. The massless case would correspond to a non-compact Coulomb branch and eventual singularity of the SCFT.

In order to address the above question we need to find the low energy degrees of freedom which couple to the gauge multiplet corresponding to $A_{r}$.

The fields charged under $A_{r}$ are the hypermultiplets $\chi_{a \bar{b}^{\prime}}, \chi_{a^{\prime} \bar{b}}, Y_{i\left(a \bar{a}^{\prime}\right)}^{(1)}, Y_{i\left(a^{\prime} \bar{a}\right)}^{(1)}$, $Y_{i\left(b b^{\prime}\right)}^{(5)}, Y_{i\left(b^{\prime} \bar{b}\right)}^{(5)}$ and the vector multiplets $\phi_{m}^{(1) a \bar{a}^{\prime}}, \phi_{m}^{(1) a^{\prime} \bar{a}}, \phi_{i}^{(5) b b^{\prime}}, \phi_{m}^{(5) b^{\prime} \bar{b}}$. In order to find out which of these are massless, we look at the following terms in the Lagrangian of $U\left(Q_{1}\right) \times U\left(Q_{5}\right)$ gauge theory:

$$
\begin{aligned}
L & =L_{1}+L_{2}+L_{3}+L_{4} \\
L_{1} & =\chi^{\dagger} \phi_{m}^{\dagger} \phi_{m} \chi \\
L_{2} & =\chi^{\dagger} \phi_{m}^{\dagger} \phi_{m} \chi_{a_{1} \bar{b}_{3}} \\
L_{3} & =\operatorname{Tr}\left(\left[Y_{i}^{(1)}, Y_{j}^{(1)}\right]\left[Y_{i}^{(1)}, Y_{j}^{(1)}\right]\right) \\
L_{4} & =\operatorname{Tr}\left(\left[Y_{i}^{(5)}, Y_{j}^{(5)}\right]\left[Y_{i}^{(5)}, Y_{j}^{(5)}\right]\right)
\end{aligned}
$$

The terms $L_{1}$ and $L_{2}$ originate from terms of the type $\left|A_{M} \chi\right|^{2}$ where $A_{M} \equiv\left(A_{\mu}, \phi_{m}\right)$ is the $(4,4)$ vector multiplet in two dimensions. The terms $L_{3}$ and $L_{4}$ arise from commutators of gauge fields in compactified directions.

The fields $Y$ are in general massive. The reason is that the traces $y_{i}^{(1)} \equiv$ $\operatorname{Tr}\left(Y_{i(a \bar{a})}^{(1)}\right)$, representing the centre-of-mass position in the $T^{4}$ of $q_{1}$ D1 branes, and $y_{i}^{\prime(1)} \equiv \operatorname{Tr}\left(Y_{i\left(a^{\prime} \bar{a}^{\prime}\right)}^{(1)}\right)$, representing the centre-of-mass position in the $T^{4}$ of $Q_{1}^{\prime} \mathrm{D} 1$ branes, are neutral and will have vev's which are generically separated (the centres of mass can be separated in the torus even when they are on top of each other in physical space). The mass of $Y_{i\left(a \bar{a}^{\prime}\right)}^{(1)}, Y_{i\left(a^{\prime} \bar{a}\right)}^{(1)}$ can be read off from the term $L_{3}$ in (7.29), to be proportional to $\left(y^{(1)}-y^{\prime(1)}\right)^{2}$ Similarly the mass of $Y_{i\left(\left(b^{\prime}\right)\right.}^{(5)}, Y_{i\left(b \bar{b}^{\prime}\right)}^{(5)}$ is proportional to $\left(y^{(5)}-y^{\prime(5)}\right)^{2}$ (as can be read off from the term $L_{4}$ in (7.29)) where $y^{(5)}$ and $y^{\prime(5)}$ are the centers of mass of the $Q_{5}$ D5 branes and $Q_{5}^{\prime}$ D5 branes along the direction of the dual four torus $\hat{T}^{4}$. (At special points when their centres of mass coincide, these fields become massless. The analysis for these cases can also be carried out by incorporating these fields in (7.33)-(7.35), with no change in the conclusion) The fields $\phi_{m}^{(1) a \bar{a}^{\prime}}, \phi_{m}^{(1) a^{\prime} \bar{a}}$ are also massive. Their masses can be read off from the $L_{1}$ in (7.29). Specifically they arise from the following terms

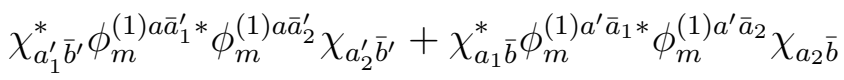

where $a_{i}$ run from $1, \ldots q_{1}$ and $a_{i}^{\prime}$ run form $1, \ldots Q_{1}^{\prime}$. These terms show that their masses are proportional to the expectation values of the hypers $\chi_{a \bar{b}}$ and $\chi_{a^{\prime} \bar{b}^{\prime}}$. 
Similarly the terms of $L_{2}$ in $(7.29)$

$$
\chi_{a^{\prime} \bar{b}_{1}^{\prime}}^{*} \phi_{m}^{(5) b_{1}^{\prime} b *} \phi_{m}^{(5) b_{2}^{\prime} \bar{b}} \chi_{a^{\prime} \bar{b}_{2}^{\prime}}+\chi_{a \bar{b}_{1}}^{*} \phi_{m}^{(5) b_{1} \bar{b}^{\prime} *} \phi_{m}^{(5) b_{2} \bar{b}^{\prime}} \chi_{a \bar{b}_{2}}
$$

show that the fields $\phi_{m}^{(5) b \bar{b}^{\prime}} \phi_{m}^{(5) b^{\prime} \bar{b}}$ are massive with masses proportional to the expectation values of the hypers $\chi_{a \bar{a}}$ and $\chi_{a^{\prime} \bar{b}^{\prime}}$. In the above equation $b_{i}$ take values from $1, \ldots, q_{5}$ and $b_{i}^{\prime}$ take values from $1, \ldots, Q_{5}^{\prime}$. Note that these masses remain non-zero even in the limit when the $\left(q_{1}, q_{5}\right)$ and $\left(Q_{1}^{\prime}, Q_{5}^{\prime}\right)$ are on the verge of separating.

Thus the relevant degrees of freedom describing the splitting process is a $1+$ 1 dimensional $U(1)$ gauge theory of $A_{r}$ with $(4,4)$ supersymmetry. The matter content of this theory consists of hypermultiplets $\chi_{a \bar{b}^{\prime}}$ with charge +1 and $\chi_{a^{\prime} \bar{b}}$ with charge -1 . This theory consists of totally $q_{1} Q_{5}^{\prime}+q_{5} Q_{1}^{\prime}$ hypers. We define the individual components of hypers as the following doublets

$$
\chi_{a \bar{b}^{\prime}}=\left(\begin{array}{c}
A_{a \bar{b}^{\prime}} \\
B_{a \bar{b}^{\prime}}^{\dagger}
\end{array}\right) \quad \chi_{a^{\prime} \bar{b}}=\left(\begin{array}{c}
A_{a^{\prime} \bar{b}} \\
B_{a^{\prime} \bar{b}}^{\dagger}
\end{array}\right)
$$

Let us now describe the dynamics of the splitting process. This is given by analyzing the hypermultiplet moduli space of the effective theory described above with the help of the D-term equations:

$$
\begin{aligned}
A_{a \bar{b}^{\prime}} A_{a \bar{b}^{\prime}}^{*}-A_{a^{\prime} \bar{b}} A_{a^{\prime} \bar{b}}^{*}-B_{b^{\prime} \bar{a}} B_{b^{\prime} \bar{a}}^{*}+B_{b \bar{a}^{\prime}} B_{b \bar{a}^{\prime}}^{*} & =0 \\
A_{a \bar{b}^{\prime}} B_{b^{\prime} \bar{a}}-A_{a^{\prime} \bar{b}} B_{b \bar{a}^{\prime}} & =0
\end{aligned}
$$

In the above equations the sum over $a, b, a^{\prime}, b^{\prime}$ is understood. These equations are generalized version of (7.20) discussed for the $R^{4} / Z_{2}$ singularity in Section 4.1. At the origin of the Higgs branch where the classical moduli space meets the Coulomb branch this linear sigma model would flow to an infrared conformal field theory which is singular. The reason for this is the same as for the $R^{4} / Z_{2}$ case. The linear sigma model contains the following term in the superpotential

$$
V=\left(A_{a \bar{b}^{\prime}} A_{a \bar{b}^{\prime}}^{*}+A_{a^{\prime} \bar{b}} A_{a^{\prime} \bar{b}}^{*}+B_{b^{\prime} \bar{a}} B_{b^{\prime} \bar{a}}^{*}+B_{b \bar{a}^{\prime}} B_{b \bar{a}^{\prime}}^{*}\right)\left(\varphi_{1}^{2}+\varphi_{2}^{2}+\varphi_{3}^{2}+\varphi_{4}^{2}\right)
$$

As in the discussion of the $R^{4} / Z_{2}$ case, at the origin of the hypermultiplet moduli space the flat direction of the Coulomb branch leads to a ground state which is not normalizable. This singularity can be avoided by deforming the D-term equations by the Fayet-Iliopoulos terms (cf. Eqns. (4.14),(4.15) and footnote (17)):

$$
\begin{array}{r}
A_{a \bar{b}^{\prime}} A_{a \bar{b}^{\prime}}^{*}-A_{a^{\prime} \bar{b}} A_{a^{\prime} \bar{b}}^{*}-B_{b^{\prime} \bar{a}} B_{b^{\prime} \bar{a}}^{*}+B_{b \bar{a}^{\prime}} B_{b \bar{a}^{\prime}}^{*}=r_{3} \\
A_{a \bar{b}^{\prime}} B_{b^{\prime} \bar{a}}-A_{a^{\prime} \bar{b}} B_{b \bar{a}^{\prime}}=r_{1}+i r_{2}
\end{array}
$$

We note here that the Fayet-Iliopoulos terms break the relative $U(1)$ under discussion and the gauge field becomes massive. The reason is that the D-terms with the 
Fayet-Iliopoulos do not permit all $A, B$ 's in the above equation to simultaneously vanish. At least one of them must be non-zero. As these $A, B$ 's are charged under the $U(1)$, the non-zero of value of $A, B$ gives mass to the vector multiplet. This can be seen from the potential (7.34). The scalars of the vector multiplet becomes massive with the mass proportional to the vev's of $A, B$. Thus the relative $U(1)$ is broken.

The singularity associated with the non-compact Coulomb branch can also be avoided by turning on the $\theta$ term, the mechanism being similar to the one discussed in the previous subsection. If any of the 3 Fayet-Iliopoulos D-terms or the $\theta$ term is turned on, the flat directions of the Coulomb branch are lifted, leading to normalizable ground state is of the Higgs branch. This prevents the breaking up of the $\left(Q_{1}, Q_{5}\right)$ system to subsystems. Thus we see that the 4 parameters which resolve the singularity of the $\mathcal{N}=(4,4)$ SCFT on $\mathcal{M}$ make their appearance in the gauge theory as the Fayet-Iliopoulos terms and the theta term.

It would be interesting to extract the singularity structure of the gauge theory of the D1-D5 system through mappings similar to (7.21)- $(\sqrt{7.24})^{19}$.

The case $\left(Q_{1}, Q_{5}\right) \rightarrow\left(Q_{1}-1, Q_{5}\right)+(1,0)$ : splitting of a single D1 brane

It is illuminating to consider the special case in which $1 \mathrm{D} 1$ brane splits off from the bound state $\left(Q_{1}, Q_{5}\right)$. The effective dynamics is again described in terms of a $U(1)$ gauge theory associated with the relative separation between the single D1-brane and the bound state $\left(Q_{1}-1, Q_{5}\right)$. The massless hypermultiplets charged under this $U(1)$ correspond to open strings joining the single D1-brane with the D5-branes and are denoted by

$$
\chi_{b^{\prime}}=\left(\begin{array}{c}
A_{b^{\prime}} \\
B_{b^{\prime}}^{\dagger}
\end{array}\right)
$$

The D-term equations, with the Fayet-Iliopoulos terms, become in this case

$$
\sum_{b^{\prime}=1}^{Q_{5}}\left(\left|A_{b^{\prime}}\right|^{2}-\left|B_{b^{\prime}}\right|^{2}\right)=r_{3}, \quad \sum_{b^{\prime}=1}^{Q_{5}} A_{b^{\prime}} B_{b^{\prime}}=r_{1}+i r_{2}
$$

while the potential is

$$
V=\left[\sum_{b^{\prime}=1}^{Q_{5}}\left(\left|A_{b^{\prime}}\right|^{2}+\left|B_{b^{\prime}}\right|^{2}\right)\right]\left(\varphi_{1}^{2}+\varphi_{2}^{2}+\varphi_{3}^{2}+\varphi_{4}^{2}\right)
$$

In this simple case it is easy to see that the presences of the Fayet-Iliopoulos terms in (7.37) ensures that all $A, B$ 's do not vanish simultaneously. The vev's of $A, B$ gives mass to the $\varphi$ 's. Thus the relative $U(1)$ is broken when the Fayet-Iliopoulos

\footnotetext{
${ }^{19}$ The singularity structure for a $U(1)$ theory coupled to $N$ hypermultiplets has been obtained in 88
} 
term is not zero. The D-term equations above agree with those in [114 which discusses the splitting of a single D1-brane. It is important to emphasize that the potential and the D-term equations describe an effective dynamics in the classical background corresponding to the $\left(Q_{1}-1, Q_{5}\right)$ bound state. This corresponds to the description in 114] of the splitting process in an $\mathrm{AdS}_{3}$ background which represents a mean field of the above bound state.

\subsection{Dynamics of the decay of the D1-D5 system from gauge theory}

We have seen in Section 7.3 that the effective theory describing the dynamics of the splitting of the $\left(Q_{1}, Q_{5}\right)$ system to subsystems $\left(q_{1}, q_{5}\right)$ and $\left(Q_{1}^{\prime}, Q_{5}^{\prime}\right)$ is $(4,4), U(1)$ super Yang-Mills coupled to $\left.q_{1} Q_{5}^{\prime}+q_{5} Q_{1}^{\prime}\right)$ hypermultiplets. The SCFT which this gauge theory flows in the infra-red is singular if the Fayet-Iliopoulos terms and the theta term is set to zero. The description of the superconformal theory of the Higgs branch of a $U(1)$ gauge theory with $(4,4)$ supersymmetry and $N$ hypermultiplets near the singularity was found in [145. The Higgs branch near the singularity was expressed in the Coulomb variables. The SCFT near the singularity was derived using the R symmetry of the Higgs branch. It consists of a bosonic $S U(2)$ WessZumino-Witten model at level $N-2$, four free fermions and a linear dilaton with background charge given by

$$
Q=\sqrt{\frac{2}{N}}(N-1)
$$

The central charge of this SCFT is $6(N-1)$. Using this result for the $U(1)$ theory describing the splitting we get a background charge for the linear dilaton given by

$$
Q_{\text {GaugeTheory }}=\sqrt{\frac{2}{q_{1} Q_{5}^{\prime}+q_{5} Q_{1}^{\prime}}}\left(q_{1} Q_{5}^{\prime}+q_{5} Q_{1}^{\prime}-1\right)
$$

For large $Q_{1}^{\prime}$ and $Q_{5}^{\prime}$ we see from the above equation and $(7.18)$ that $Q_{\text {GaugeTheory }}=$ $Q_{\text {SUGRA }}$

Consider the case of a single string splitting off the D1-D5 bound state, then the linear dilaton theory relevant for this decay has a background charge of $Q=$

$\sqrt{\frac{2}{Q_{5}}}\left(Q_{5}-1\right)$. This effective theory is called the long string. On performing an S-duality transformation this long D-string turns into a long fundamental string. This argument demonstrates the existence of long fundamental strings in the Sdual of the near horizon geometry of the D1-D5 system. We will discuss these solutions in detail in section 10.

\subsection{The symmetric product}

From the arguments of this section we see that the free field orbifold conformal field theory on $\mathcal{M}$ does not correspond to the D1-D5 system given by the supergravity 
solution in (2.70). This solution does not have any moduli turned on. We saw in this section that in the absence of moduli the SCFT is singular. The effective theory near the singularities does not just depend on the product $Q_{1} Q_{5}$. For instance the theory near the singularity corresponding to the decay of a single D1 brane is characterized by a background charge of $\sqrt{\frac{2}{Q_{5}}}\left(Q_{5}-1\right)$. Thus it is not clear that whether the symmetric product moduli space is connected to this singular SCFT.

In spite of this from the fact that the short multiplets of the SCFT on $\mathcal{M}$ agree with the supergravity modes on $A d S_{3} \times S^{3}$ we see that at least for calculations involving correlations functions of the short multiplets we can trust the SCFT on the symmetric product $\mathcal{M}$. The reason for this is that correlations functions involving shortmultiplets are protected by non-renormalization theorems. 


\section{The microscopic derivation of Hawking radiation}

From section 5 and section 6 we have seen that there is a one-to-one correspondence of the supergavity modes and the short mulitplets of the $\mathcal{N}=(4,4)$ SCFT on $\mathcal{M}$. In this section we use this fact to obtain a precise understanding of Hawking radiation from the D1-D5 system starting from the microscopic SCFT.

As mentioned in the introduction after extracting out the low energy, degrees of freedom of the black hole the next step towards understanding Hawking radiation is to find the coupling of these degrees of freedom to the supergravity modes. This is given by a specific SCFT operator $\mathcal{O}(z, \bar{z})$ which couples to the supergravity field $\phi$ in the form of the interaction

$$
S_{\mathrm{int}}=\mu \int d^{2} z \phi(z, \bar{z}) \mathcal{O}(z, \bar{z})
$$

where $\mu$ is the strength of the coupling. We have no first principle method of determining the operator corresponding $\mathcal{O}$ which couples with the supergravity mode as the microsopic theory is an effective theory. Therefore we appeal to symmetries to determine the operator. A coupling such as the one given in (8.1) can exist only if the operator $\mathcal{O}$ and the field $\phi$ have the same symmetries. The identification of the bulk and boundary symmetries of the D1-D5 system in section 6.3 enables the determination of the operator which couples to a given supergravity mode. Strictly speaking in the near horizon limit (2.6.2) there is no coupling of the bulk to the boundary. Here, we will assume $\alpha^{\prime}$ small but strictly not zero, so that we can discuss Hawking radiation. From the near horizon limit of the D1-D5 black hole whose metric in 5-d is given in (2.42) we can infer that the black hole is an excited state of the Ramond sector of the same SCFT as that of the unexcited D1-D5 system. Therefore the coupling (8.1) should be the same as that of the D1D5 system. The strength of the coupling $\mu$ is determined by comparing bulk and boundary two point function using the AdS/CFT correspondence for the D1-D5 system. Once the interaction in (8.1) is determined the calculation of Hawking radiation from the SCFT reduces to a purely quantum mechanical evaluation of a scattering matrix in the SCFT.

In section 8.1 an 8.2 we identify the D1-D5 black hole as an excited state in the Ramond sector of the SCFT of the D1-D5 system. We show how the entropy of the D1-D5 black hole matches with this excited state in the SCFT. In section 8.3 we determine the coupling of the minimal scalar corresponding to the metric fluctuation of the torus to the SCFT operator. In section 8.4 we review the formulation of the absorption cross-section calculation from SCFT as an evaluation of the thermal Green's function of the operators $\mathcal{O}$ corresponding to the supergravity field $\phi$. We then evaluate the absorption cross-section from SCFT and show that it agrees with the one evaluated from supergravity including all the graybody factors. 
In section 8.6 we address the Hawking radiation of fixed scalars from the SCFT point of view. We show that fixing the SCFT operators using symmetries resolves the disagreement observed in [104] between the 'effective string' calculation of the Hawking radiation and the supergravity calculation. Finally in section 8.7 we outline how Hawking radiation of the intermediate scalars also can be determined from the SCFT.

\subsection{Near horizon limit and Fermion boundary conditions}

The near-horizon geometry of the D1-D5 black hole is described in detail in Sec. 2.6.2. We see from the remark (b) at the end of that section that the boundary condition for fermions in the BTZ case is periodic; this implies that the SCFT relevant for the D1-D5 black hole with Kaluza-Klein momentum $N=0$ is the Ramond vacuum of the $\mathcal{N}=(4,4)$ SCFT on the orbifold $\mathcal{M}$. The microscopic states corresponding to the general D1-D5 black hole are states with $L_{0} \neq 0$ and $\bar{L}_{0} \neq 0$ excited over the Ramond vaccum of the $\mathcal{N}=(4,4)$ SCFT on the orbifold $\mathcal{M}$. In the $A d S_{3}$ case, the fermion boundary condition is antiperiodic; therefore the appropriate SCFT is that of the NS sector.

\subsection{The black hole state}

As we have seen, the general non-extremal black hole will have Kaluza-Klein excitations along both the directions on the $S^{1}$. In the SCFT on $\mathcal{M}$, it is represented by states with $L_{0} \neq 0$ and $\bar{L}_{0} \neq 0$ over the Ramond vacuum. The black hole is represented by a density matrix (cf. Eq. (1.14))

$$
\rho=\frac{1}{\Omega} \sum_{\{i\}}|i\rangle\langle i|
$$

The states $|i\rangle$ belongs to the various twisted sectors of the orbifold theory. They satisfy the constraint

$$
L_{0}=\frac{N_{L}}{Q_{1} Q_{5}} \quad \bar{L}_{0}=\frac{N_{R}}{Q_{1} Q_{5}}
$$

We have suppressed the index which labels the vacuum. $\Omega$ is the volume of the phase space in the microcanonical ensemble. It can be seen that the maximally twisted sector of the orbifold gives rise to the dominant contribution to the sum in (8.2) over the various twisted sectors. The maximally twisted sector is obtained by the action of the twist operator $\sum^{\left(Q_{1} Q_{5}-1\right) / 2}$ on the Ramond vacuum. From the

OPE's in (5.39) we see that the twist operator $\sum^{\left(Q_{1} Q_{5}-1\right) / 2}$ introduces a cut in the complex plane such that

$$
X_{A}\left(e^{2 \pi i} z, e^{-2 \pi i} \bar{z}\right)=X_{A+1}(z, \bar{z})
$$

Thus this changes the boundary conditions of the bosons and the fermions. Again from the OPEs in (5.39) one infers that the excitations like $\partial \phi_{1}\left|\sum^{\left(Q_{1} Q_{5}-1\right) / 2}\right\rangle$ over 
the maximally twisted sector have modes in units of $1 / Q_{1} Q_{5}$. A simple way of understanding that the maximally twisted sector has modes in units of $1 /\left(Q_{1} Q_{5}\right)$ is to note that the boundary conditions in (8.4) imply that $X_{A}(z, \bar{z})$ is periodic with a period of $2 \pi Q_{1} Q_{5}$. This forces the modes to be quantized in units of $1 /\left(Q_{1} Q_{5}\right)$.

We now show that the maximally twisted sector can account for the entire entropy of the black hole. The entropy of the D1-D5 black hole can be written as

$$
S_{S U G R A}=2 \pi \sqrt{N_{L}}+2 \pi \sqrt{N_{R}}
$$

Using Cardy's formula, the degeneracy of the states in the maximally twisted sector with $L_{0}=N_{L} / Q_{1} Q_{5}$ and $\bar{L}_{0}=N_{R} / Q_{1} Q_{5}$ is given by

$$
\Omega=e^{2 \pi \sqrt{N_{L}}+2 \pi \sqrt{N_{R}}}
$$

By the Boltzmann formula,

$$
S(\text { maximally twisted })=2 \pi \sqrt{N_{L}}+2 \pi \sqrt{N_{R}}
$$

Thus the maximally twisted sector entirely accounts for the D1-D5 black hole entropy. $N_{L}$ and $N_{R}$ are multiples of $Q_{1} Q_{5}$ due to the orbifold projection. Therefore, the entropy can be written as

$$
S=2 \pi \sqrt{N_{L} Q_{1} Q_{5}}+2 \pi \sqrt{N_{R} Q_{1} Q_{5}}
$$

With this understanding, we restrict the calculations of Hawking radiation and absorption cross-section only to the maximally twisted sector. The probability amplitude for the Hawking process is given by

$$
P=\frac{1}{\Omega} \sum_{f, i}\left|\left\langle f\left|S_{i n t}\right| i\right\rangle\right|^{2}
$$

where $|f\rangle$ denotes the final states the black hole can decay into. We have averaged over the initial states in the microcanonical ensemble.

It is more convenient to work with the canonical ensemble. We now discuss the method of determining the temperature of the canonical ensemble. Consider the generating function

$$
Z=\operatorname{Tr}_{R}\left(e^{-\beta_{L} E_{0}} e^{-\beta_{R} \bar{E}_{0}}\right)
$$

where the trace is evaluated over the Ramond states in the maximally twisted sector. $E_{0}$ and $\bar{E}_{0}$ are energies of the left and the right moving modes.

$$
E_{0}=\frac{L_{0}}{R_{5}}, \quad \bar{E}_{0}=\frac{\bar{L}_{0}}{R_{5}}
$$

From the generating function $\mathrm{Z}$ in (8.10) we see that the coefficient of $e^{-\left(\beta_{L} N_{L}\right) /\left(Q_{1} Q_{5} R_{5}\right)}$ and $e^{-\left(\beta_{R} N_{R}\right) /\left(Q_{1} Q_{5} R_{5}\right)}$ is the degeneracy of the states with $L_{0}=N_{L} / Q_{1} Q_{5}$ and 
$\bar{L}_{0}=N_{R} / Q_{1} Q_{5}$ corresponding to the D1-D5 black hole. A simple way to satisfy this constraint is to choose $\beta_{L}$ and $\beta_{R}$ such that $\mathrm{Z}$ is peaked at this value of $L_{0}$ and $\bar{L}_{0}$.

Evaluating the trace one obtains

$$
Z=\prod_{n=1}^{\infty}\left(\frac{1+e^{-\left(\beta_{L} n\right) /\left(Q_{1} Q_{5} R_{5}\right)}}{1-e^{-\left(\beta_{L} n\right) /\left(Q_{1} Q_{5} R_{5}\right)}}\right)^{4}\left(\frac{1+e^{-\left(\beta_{R} n\right) /\left(Q_{1} Q_{5} R_{5}\right)}}{1-e^{-\left(\beta_{R} n\right) /\left(Q_{1} Q_{5} R_{5}\right)}}\right)^{4}
$$

Then

$$
\begin{aligned}
\ln Z= & 4\left[\sum_{n=1}^{\infty} \ln \left(1+e^{-\beta_{L} n / Q_{1} Q_{5} R_{5}}\right)-\sum_{n=1}^{\infty} \ln \left(1-e^{-\beta_{L} n / Q_{1} Q_{5} R_{5}}\right)\right] \\
& +4\left[\sum_{n=1}^{\infty} \ln \left(1+e^{-\beta_{R} n / Q_{1} Q_{5} R_{5}}\right)-\sum_{n=1}^{\infty} \ln \left(1-e^{-\beta_{R} n / Q_{1} Q_{5} R_{5}}\right)\right]
\end{aligned}
$$

We can evaluate the sum by approximating it by an integral given by

$$
\ln Z=4 Q_{1} Q_{5} R_{5} \int_{0}^{\infty} d x\left[\ln \left(\frac{1+e^{-\beta_{L} x}}{1-e^{-\beta_{L} x}}\right)+\ln \left(\frac{1+e^{-\beta_{R} x}}{1-e^{-\beta_{R} x}}\right)\right]
$$

From the partition function in (8.10) we see that

$$
-\frac{\partial \ln Z}{\partial \beta_{L}}=\frac{\left\langle N_{L}\right\rangle}{Q_{1} Q_{5} R_{5}} \text { and }-\frac{\partial \ln Z}{\partial \beta_{R}}=\frac{\left\langle N_{R}\right\rangle}{Q_{1} Q_{5} R_{5}}
$$

where $\langle\cdot\rangle$ indicates the average value of $N_{L}$ and $N_{R}$. As the distribution is peaked at $N_{L}$ and $N_{R}$ we assume that $\left\langle N_{L}\right\rangle=N_{L}$ and $\left\langle N_{L}\right\rangle=N_{R}$. Using (8.15) we obtain

$$
\frac{Q_{1} Q_{5} R_{5} \pi^{2}}{\beta_{L}^{2}}=\frac{N_{L}}{Q_{1} Q_{5} R_{5}} \quad \text { and } \quad \frac{Q_{1} Q_{5} R_{5} \pi^{2}}{\beta_{R}^{2}}=\frac{N_{R}}{Q_{1} Q_{5} R_{5}}
$$

Thus

$$
T_{L}=\frac{1}{\beta_{L}}=\frac{\sqrt{N_{L}}}{\pi R_{5} Q_{1} Q_{5}} \text { and } T_{R}=\frac{1}{\beta_{R}}=\frac{\sqrt{N_{R}}}{\pi R_{5} Q_{1} Q_{5}}
$$

Above we have introduced a left temperature $T_{L}$ and a right temperature $T_{R}$ corresponding to the left and the right moving excitations of the SCFT to pass over to the canonical ensemble. To see that the temperatures $T_{L}, T_{R}$ defined here are the same as in (4.29), note that the oscillator numbers $N_{L}, N_{R}$ are $Q_{1} Q_{5}$ times the oscillator numbers that enter (4.29). The reason is that in (4.29) we defined $N_{L}, N_{R}$ simply as the eigenvalues of $L_{0}, \overline{L_{0}}$; in this secion we are working with the maximally twisted sectors which have fractional oscillator numbers, thus to reach the same energy we have to work with oscillator numbers which are $Q_{1} Q_{5}$ times larger.

As mentioned before (cf. (4.30) ) the temperature of the combined system (conjugate to $E_{L}+E_{R}$ ), to be identified as the Hawking temperature, is given by

$$
1 / T_{H}=\frac{1}{2}\left(1 / T_{L}+1 / T_{R}\right)
$$




\subsection{The coupling with the bulk fields for the D1-D5 black hole}

In section 6 we showed that there is a one to one map between the supergravity fields on $A d S_{3} \times S^{3}$ and the short multiplets of the $\mathcal{N}=(4,4) \operatorname{SCFT}$ on $\mathcal{M}$. Therefore in principle we can determine $\mathcal{O}$ for each bulk field $\phi$ in (8.1) just by matching the symmetries of the operator and the field. But as we mentioned above that the black hole is represented by an excited state in the Ramond sector and not for the Neveu-Schwarz sector which corresponds to the $A d S_{3}$ boundary conditions. The couplings determined in the Neveu-Schwarz sector of the SCFT do not change in the Ramond sector as interaction terms do not depend on whether one is in the Ramond sector or the Neveu-Schwarz sector. Therefore we can continue to use these couplings for the D1-D5 black hole. The scaling dimension of an operator is given by operator product expansions(OPEs) with the stress energy tensor. Since OPEs are local relations, they do not change on going from the Neveu-Schwarz sector to the Ramond sector, the same can be said of the R-charge of the operator. In section 8. we will see the calculation of Hawking radiation from the SCFT just depends on the scaling dimension and the R-charge of the operator. Since this is invariant whether one is in the Ramond sector or the Neveu-Schwarz sector the operator $\mathcal{O}$ is the same as the one identified using $A d S_{3}$ as the near horizon geometry.

\subsection{Determination of the strength of the coupling $\mu$}

Before we perform the calculation of Hawking radiation/absorption cross-section from the SCFT corresponding to the D1-D5 black hole it is important to determine the strength of the coupling $\mu$ in (8.1). In this section we will determine $\mu$ for the case of minimal scalars $h_{i j}{ }^{20}$ In (6.16) we have identified the SCFT operator corresponding to these fields of the supergravity. The SCFT operator is given by

$$
\mathcal{O}^{i j}(z, \bar{z})=\partial x_{A}^{\{i}(z, \bar{z}) \bar{\partial} x_{A}^{j\}}(z, \bar{z})-\frac{1}{4} \delta^{i j} \partial x_{A}^{k} \bar{\partial} x_{A}^{k}(z, \bar{z})
$$

Let us suppose the background metric of the torus $T^{4}$ is $g_{i j}=\delta_{i j}$. The interaction Lagrangian of the SCFT with the fluctuation $h_{i j}$ is given by

$$
S_{\text {int }}=\mu T_{\text {eff }} \int d^{2} z\left[h_{i j} \partial x_{A}^{i} \bar{\partial} x_{A}^{j}\right]
$$

The effective string tension $T_{\text {eff }}$ of the conformal field theory, which also appears in the free part of the action

$$
S_{0}=T_{\text {eff }} \int d^{2} z\left[\partial_{z} x_{A}^{i} \partial_{\bar{z}} x_{i, A}+\text { fermions }\right]
$$

has been discussed in [101, 48, 111]. The specific value of $T_{\text {eff }}$ is not important for the calculation of the $S$-matrix for absorption or emission, since the factor just

\footnotetext{
${ }^{20}$ From now on $h_{i j}$ will denote the traceless part of the metric fluctuations of $T^{4}$.
} 
determines the normalization of the two-point function of the operator $\mathcal{O}^{i j}(z, \bar{z})$. In this section we will argue that the constant $\mu=1$.

A direct string theory computation would of course provide the constant $\mu$ as well (albeit at weak coupling). This would be analogous to fixing the normalization of the Dirac-Born-Infeld action for a single D-brane by comparing with one-loop open string diagram [109. However, for a large number and more than one type of D-branes it is a difficult proposition and we will not attempt to pursue it here. Fortunately, the method of symmetries using the AdS/CFT employed for determining the operator $\mathcal{O}$ helps us determine the value of $\mu$ as well. For the latter, however, we need to use the more quantitative version 62, 63 of the Maldacena conjecture. We will see below that for this quantitative conjecture to be true for the two-point function (which can be calculated independently from the $\mathcal{N}=(4,4)$ SCFT and from supergravity) we need $\mu=1$.

We will see that the above normalization leads to precise equality between the absorption cross-sections (and consequently Hawking radiation rates) computed from the moduli space of the D1-D5 system and from semiclassical gravity. This method of fixing the normalization can perhaps be criticized on the ground that it borrows from supergravity and does not rely entirely on the SCFT. However, we would like to emphasize two things:

(a) We have fixed $\mu=1$ by comparing with supergravity around $\mathrm{AdS}_{3}$ background which does not have a black hole. On the other hand, the supergravity calculation of absorption cross-section and Hawking flux is performed around a black hole background represented in the near-horizon limit by the BTZ black hole. From the viewpoint of semiclassical gravity these two backgrounds are rather different. The fact that normalizing $\mu$ with respect to the former background leads to the correctly normalized absorption cross-section around the black hole background is a rather remarkable prediction.

(b) Similar issues are involved in fixing the coupling constant between the electron and the electromagnetic field in the semiclassical theory of radiation in terms of the physical electric charge, and in similarly fixing the gravitational coupling of extended objects in terms of Newton's constant. These issues too are decided by comparing two-point functions of currents with Coulomb's or Newton's laws respectively. In the present case the quantitative version of the AdS/CFT conjecture [62, 63] provides the counterpart of Newton's law or Coulomb's law at strong coupling. Without this the best result one can achieve is that the Hawking radiation rates computed from D1-D5 branes and from semiclassical gravity are proportional.

We should remark that fixing the normalization by the use of Dirac-BornInfeld action, as has been done previously, is not satisfactory since the DBI action is meant for single D-branes and extending it to a system of multiple D1-D5 branes does not always give the right results as we have seen in Section 8.7. The method of equivalence principle to fix the normalization is not very general and cannot be 
applied to the case of non-minimal scalars, for example.

Let us now compare the two-point function for the minimal scalar $h_{i j}$ determined from the AdS/CFT correspondence and the SCFT to determine the normalization constant $\mu$. We will discuss the more quantitative version of the AdS/CFT conjecture 62, 63] to compare the 2-point correlation function of $\mathcal{O}_{i j}$ from supergravity and SCFT.

The relation between the correlators are as follows. Let the supergravity Lagrangian be

$$
\begin{aligned}
L & =\int d^{3} x_{1} d^{3} x_{2} b_{i j, i^{\prime} j^{\prime}}\left(x_{1}, x_{2}\right) h_{i j}\left(x_{1}\right) h_{i^{\prime} j^{\prime}}\left(x_{2}\right) \\
& +\int d^{3} x_{1} d^{3} x_{2} d^{3} x_{3} c_{i j, i^{\prime} j^{\prime}, i^{\prime \prime} j^{\prime \prime}}\left(x_{1}, x_{2}, x_{3}\right) h_{i j}\left(x_{1}\right) h_{i^{\prime} j^{\prime}}\left(x_{2}\right) h_{i^{\prime \prime} j^{\prime \prime}}\left(x_{3}\right)+\ldots
\end{aligned}
$$

where we have only exhibited terms quadratic and cubic in the $h_{i j}$ 's. The coefficient $b$ determines the propagator and the coefficient $c$ is the tree-level 3-point vertex in supergravity. The coefficients $b$ and $c$ are local operators, $b$ is the kinetic operator.

The 2-point function of the $\mathcal{O}_{i j}$ 's (at large $g_{s} Q_{1}, g_{s} Q_{5}$ ) is given by 62, 63 assuming $S_{\text {int }}$ given by (8.21)

$$
\begin{gathered}
\left\langle\mathcal{O}_{i j}\left(z_{1}\right) \mathcal{O}_{i^{\prime} j^{\prime}}\left(z_{2}\right)\right\rangle \\
=2\left(\mu T_{\text {eff }}\right)^{-2} \int d^{3} x_{1} d^{3} x_{2}\left[b_{i j, i^{\prime} j^{\prime}}\left(x_{1}, x_{2}\right) K\left(x_{1} \mid z_{1}\right) K\left(x_{2} \mid z_{2}\right)\right],
\end{gathered}
$$

where $K$ is the boundary-to-bulk Green's function for massless scalars [62].

$$
K(x \mid z)=\frac{1}{\pi}\left[\frac{x_{0}}{\left(x_{0}^{2}+\left(\left|z_{x}-z\right|^{2}\right)\right.}\right]^{2}
$$

We use complex $z$ for coordinates of the SCFT, and $x=\left(x_{0}, z_{x}\right)$ for the Poincaré coordinates of bulk theory.

\subsubsection{Evaluation of the tree-level vertices in supergravity}

We begin with the bosonic sector of Type IIB supergravity. The Lagrangian is (we follow the conventions of 148 )

$$
\begin{aligned}
I & =I_{\mathrm{NS}}+I_{\mathrm{RR}} \\
I_{\mathrm{NS}} & =-\frac{1}{2 k_{10}^{2}} \int d^{10} x \sqrt{-G}\left[e^{-2 \phi}\left(R-4(d \phi)^{2}+\frac{1}{12}\left(d B_{N S}\right)^{2}\right)\right] \\
I_{\mathrm{RR}} & =-\frac{1}{2 k_{10}^{2}} \int d^{10} x \sqrt{-G}\left(\sum_{n=3,7, \ldots} \frac{1}{2 n !}\left(H^{n}\right)^{2}\right)
\end{aligned}
$$

with $k_{10}^{2}=64 \pi^{7} g_{s}^{2} \alpha^{4}$. We use $\hat{M}, \hat{N} \ldots$ to denote 10 dimensional indices, $i, j, \ldots$ to denote coordinates on the torus $T^{4}, M, N \ldots$ to denote the remaining 6 dimensions 
and $\mu, \nu, \ldots$ to denote coordinates on the $\mathrm{AdS}_{3}$. We have separately indicated the terms depending on Neveu-Schwarz Neveu-Schwarz and Ramond-Ramond backgrounds.

Our aim will be to obtain the Lagrangian of the minimally coupled scalars corresponding to the fluctuations of the metric of the $T^{4}$ in the D1-D5 -brane system. We will find the Lagrangian up to cubic order in the near horizon limit. Let us first focus on $I_{\mathrm{NS}}$. We substitute the values of the background fields of the D1-D5 system in the Type IIB Lagrangian with the following change in the metric (cf. Eq. (3.3))

$$
f_{1}^{\frac{1}{2}} f_{5}^{-\frac{1}{2}} \delta_{i j} \rightarrow f_{1}^{\frac{1}{2}} f_{5}^{-\frac{1}{2}}\left(\delta_{i j}+h_{i j}\right)
$$

where $h_{i j}$ are the minimally coupled scalars with trace zero. These scalars are functions of the 6 dimensional coordinates. Retaining the terms upto $O\left(h^{3}\right)$ and ignoring the traces, the Lagrangian can be written as

$$
I_{\mathrm{NS}}=-\frac{V_{4}}{2 k_{10}^{2}} \int d^{6} x \sqrt{-G} \frac{G^{M N}}{4}\left[\partial_{M} h_{i j} \partial_{N} h_{i j}+\partial_{M}\left(h_{i k} h_{k j}\right) \partial_{N} h_{i j}\right]
$$

In the above equation we have used the near horizon limit and $V_{4}$ is the volume of the $T^{4}$. It is easy to see that to $O\left(h^{2}\right)$, the minimally coupled scalars do not mix with any other scalars. These minimally coupled scalars are all massless (see the first line in (6.15)). Therefore to $O\left(h^{2}\right)$ they do not mix with any other scalars which are all massive. For our purpose of computing the two point function using the AdS/CFT correspondence it is sufficient to determine the tree-level action correct to $O\left(h^{2}\right)$. The metric $G_{M N}$ near the horizon is (writing $r$ for $U$ in Eq. (2.71), and including a factor of $\alpha^{\prime}$ )

$$
d s^{2}=\frac{r^{2}}{R^{2}}\left(-d x_{0}^{2}+d x_{5}^{2}\right)+\frac{R^{2}}{r^{2}} d r^{2}+R^{2} d \Omega_{3}^{2}
$$

We make a change of variables to the Poincaré coordinates by substituting

$$
z_{0}=\frac{R}{r}, z_{1}=\frac{x_{0}}{R}, z_{2}=\frac{x_{5}}{R}
$$

The metric becomes

$$
d s^{2}=R^{2} \frac{1}{z_{0}^{2}}\left(d z_{0}^{2}-d z_{1}^{2}+d z_{2}^{2}\right)+R^{2} d \Omega_{3}^{2} .
$$

Here $R=\sqrt{\alpha^{\prime}}\left(g_{6}^{2} Q_{1} Q_{5}\right)^{1 / 4}$ is the radius of curvature of $A d S_{3}$ (also of the $S^{3}$ ) (see (2.73)). For $s$-waves the minimal scalars do not depend on the coordinates of the $S^{3}$. Finally, in Poincaré coordinates $I_{\mathrm{NS}}$ (correct to cubic order in $h$ ) can be written as

$$
I_{\mathrm{NS}}=-\frac{V_{4}}{8 k_{10}^{2}} R^{3} V_{S^{3}} \int d^{3} z \sqrt{-g} g^{\mu \nu}\left[\partial_{\mu} h_{i j} \partial_{\nu} h_{i j}+\partial_{\mu}\left(h_{i k} h_{k j}\right) \partial_{\nu} h_{i j}\right]
$$


where $V_{S^{3}}=2 \pi^{2}$, the volume of a three-sphere of unit radius.

Now we would like to show that to all orders in h, $I_{R R}=0$ in the near horizon geometry. The relevant terms in our case are

$$
I_{R R}=-\frac{1}{4 \times 3 ! k_{10}^{2}} \int d^{10} x \sqrt{-G} H_{\hat{M} \hat{N} \hat{O}} H^{\hat{M} \hat{N} \hat{O}} .
$$

We substitute the values of $B$ due to the magnetic and electric components of the Ramond-Ramond charges and the value of $G$. The contribution from the electric part of $B^{\prime}$, after going to the near-horizon limit and performing the integral over $S^{3}$ and $T^{4}$ is

$$
\frac{V_{4}}{4 k_{10}^{2}} R V_{S^{3}} \int d^{3} z \sqrt{-g} \sqrt{\operatorname{det}\left(\delta_{i j}+h_{i j}\right)}
$$

The contribution of the magnetic part of $B^{\prime}$ in the same limit is

$$
-\frac{V_{4}}{4 k_{10}^{2}} R V_{S^{3}} \int d^{3} z \sqrt{-g} \sqrt{\operatorname{det}\left(\delta_{i j}+h_{i j}\right)}
$$

We note that the contribution of the electric and the magnetic parts cancel giving no couplings for the minimal scalars to the Ramond-Ramond background. Therefore the tree-level supergravity action correct to cubic order in $h$ is given by ${ }^{21}$

$$
I=-\frac{Q_{1} Q_{5}}{16 \pi} \int d^{3} z\left[\partial_{\mu} h_{i j} \partial_{\mu} h_{i j}+\partial_{\mu}\left(h_{i k} h_{k j}\right) \partial_{\mu} h_{i j}\right]
$$

The coefficient $Q_{1} Q_{5} /(16 \pi)$ is U-duality invariant. This is because it is a function of only the integers $Q_{1}$ and $Q_{5}$. This can be tested by computing the same coefficient from the Neveu-Schwarz/fundamental string background which is related to the D1-D5 system by S-duality. The Neveu-Schwarz/fundamental string back ground also gives the same coefficient. U-duality transformations which generate $B_{N S}$ backgrounds [88] also give rise to the same coefficient.

\subsubsection{Two-point function}

The two-point function of the operator $\mathcal{O}_{i j}$ can be evaluated by substituting the value of $b_{i j, i^{\prime} j^{\prime}}\left(x_{1}, x_{2}\right)$ obtained from (8.36) into (8.24) and using the boundary-tobulk Green's function given in (8.25). On evaluating the integral in (8.24) using formulae given in 150, we find that

$$
\left\langle\mathcal{O}_{i j}(z) \mathcal{O}_{i^{\prime} j^{\prime}}(w)\right\rangle=\left(\mu T_{\mathrm{eff}}\right)^{-2} \delta_{i i^{\prime}} \delta_{j j^{\prime}} \frac{Q_{1} Q_{5}}{16 \pi^{2}} \frac{1}{|z-w|^{4}}
$$

This is exactly the value of the two-point function obtained from the SCFT described by the free Lagrangian (5.1) provided we put $\mu=1$.

\footnotetext{
${ }^{21}$ The cubic couplings of all fields in type IIB supergravity on $A d S_{3} \times S^{3} \times T^{4}$ were determined in [149].
} 
We have compared the two-point function obtained from the supergravity corresponding to the near horizon geometry of the D1-D5 system with no moduli to the orbifold SCFT. As we have argued before the orbifold SCFT corresponds to the D1-D5 system with moduli. Thus naively this comparison seems to be meaningless. On further examination we note that the coefficient $b_{i j, i^{\prime} j^{\prime}}$ in (8.24) was U-duality invariant. Since the D1-D5 system with moduli can be obtained through U-duality transformations we know that this coefficient will not change for the D1-D5 system with moduli. It is only the value of this coefficient which fixes $\mu$ to be 1 . Thus the comparison we have made is valid. It is remarkable that even at strong coupling the two-point function of $\mathcal{O}_{i j}$ can be computed from the free Lagrangian (8.22). This is consistent with the non-renormalization theorems involving the $\mathcal{N}=(4,4)$ SCFT which will be discussed in Section 9.

The choice $\mu=1$ ensures that the perturbation (8.21) of (8.22) is consistent with the perturbation implied in (8.27). We will see in the next section that this choice leads to precise equality between absorption cross-sections (consequently Hawking radiation rates) calculated from semiclassical gravity and from the D1D5 branes. The overall multiplicative constant $T_{\text {eff }}$ will not be important for the absorption cross-section calculation. This factor finally cancels off in the calculation as we will see in Section 8.6.

Higher point correlations functions in the orbifold conformal field theory were determined in [151, 152] using general methods of computing correlations functions of twist fields on symmetric product orbifolds developed by [153, 154

\subsection{Absorption cross-section as thermal Green's function}

Let us now relate the absorption cross-section of a supergravity fluctuation $\delta \phi$ to the thermal Green's function of the corresponding operator of the $\mathcal{N}=(4,4)$ SCFT on the orbifold $\mathcal{M}[155]$. The notation $\delta \phi$ implies that we are considering the supergravity field to be of the form

$$
\phi=\phi_{0}+\mu \delta \bar{\phi}
$$

where $\phi_{0}$ represents the background value and $\mu$ is the strength of the coupling.

$$
\begin{aligned}
S & =S_{0}+\int d^{2} z\left[\phi_{0}+\mu \delta \bar{\phi}\right] \mathcal{O}(z, \bar{z}) \\
& =S_{\phi_{0}}+S_{i n t}
\end{aligned}
$$

where

$$
\begin{gathered}
S_{\phi_{0}}=S_{0}+\int d^{2} z \phi_{0} \mathcal{O}(z, \bar{z}) \\
S_{\text {int }}=\mu \int d^{2} z \delta \bar{\phi} \mathcal{O}(z, \bar{z})
\end{gathered}
$$


$\mathcal{O}$ is the operator corresponding to supergravity field $\phi . \quad S_{0}$ is the Lagrangian of the SCFT which includes the deformations due to various backgrounds in the supergravity. For example, the free Lagrangian in (8.22) corresponds to the case when the field $a_{1} \chi+a_{2} C_{6789}$ in (6.15) is turned on.

We calculate the absorption of a quanta $\delta \bar{\phi}=\kappa_{5} e^{-i p x}$ corresponding to the operator $\mathcal{O}$ using the Fermi's Golden Rule. $\kappa_{5}$ is related to the five-dimensional Newton's constant $G_{5}$ and the ten-dimensional Newton's constant $G_{10}$ as

$$
\kappa_{5}^{2}=8 \pi G_{5}=\frac{8 \pi G_{10}}{V_{4} 2 \pi R_{5}}=\frac{64 \pi^{7} g_{s}^{2} \alpha^{4}}{V_{4} 2 \pi R_{5}}
$$

We see that $\kappa_{5}$ is proportional to $\alpha^{\prime 2}$. In the Maldacena limit (2.74) the coupling of the bulk fluctuation to the SCFT drops out. We retain this term for our absorption cross-section calculation. In fact we will see below that the absorption cross-section turns out to be proportional $g_{6}^{2} \sqrt{Q_{1} Q_{5}} \alpha^{\prime 2}$.

In this computation of the absorption cross-section the black hole is represented by a canonical ensemble at a given temperature. The above interaction gives the thermally averaged transition probability $\mathcal{P}$ as

$$
\mathcal{P}=\sum_{i, f} \frac{e^{-\beta \cdot p_{i}}}{Z} P_{i \rightarrow f}=\mu^{2} \kappa_{5}^{2} L t \sum_{i, f} \frac{e^{-\beta \cdot p_{i}}}{Z}(2 \pi)^{2} \delta^{2}\left(p+p_{i}-p_{f}\right)|\langle f|\mathcal{O}(0,0)| i\rangle|^{2}
$$

Here $i$ and $f$ refer to initial and final states respectively. $p_{i}, p_{f}$ refers to the initial and final momenta of these states. $L=2 \pi R_{5}$ denotes the length of the string and $t$ is the time of interaction. As we have seen in Section 8.2, the inverse temperature $\beta$ has two components $\beta_{L}$ and $\beta_{R}$. The relation of these temperatures to the parameters of the D1-D5 black hole is

$$
\beta_{L}=\frac{1}{T_{L}} \text { and } \beta_{R}=\frac{1}{T_{R}}
$$

The left moving momenta $p_{+}$and the right moving momenta $p_{-}$are in a thermal bath with inverse temperatures $\beta_{L}$ and $\beta_{R}$ respectively. $\beta \cdot p$ is defined as $\beta \cdot p=$ $\beta_{L} p_{+}+\beta_{R} p_{-} . Z$ stands for the partition function of the thermal ensemble.

The Green's function in Euclidean time is given by

$$
\mathcal{G}(-i \tau, x)=\left\langle\mathcal{O}^{\dagger}(-i \tau, x) \mathcal{O}(0,0)\right\rangle=\operatorname{Tr}\left(\rho T_{\tau}\left\{\mathcal{O}^{\dagger}(-i \tau, x) \mathcal{O}(0,0)\right\}\right)
$$

where $\rho=e^{-\beta \cdot \hat{p}} / Z$. Time ordering is defined as $T_{\tau}$ with respect to $-\operatorname{Imaginary}(t)$. This definition coincides with radial ordering on mapping the coordinate $(\tau, x)$ from the cylinder to the plane. The advantage of doing this is that the integral

$$
\int d t d x e^{i p \cdot x} \mathcal{G}(t-i \epsilon, x)=\sum_{i, f} \frac{e^{-\beta \cdot p_{i}}}{Z}(2 \pi)^{2} \delta^{2}\left(p+p_{i}-p_{f}\right)|\langle f|\mathcal{O}(0,0)| i\rangle|^{2}
$$


The Green's function $\mathcal{G}$ is determined by the two-point function of the operator $\mathcal{O}$. This is in turn determined by conformal dimension $(h, \bar{h})$ of the operator $\mathcal{O}$ and the normalization of the two-point function.

As we have to subtract out the emission probability we get the cross-section as

$$
\sigma_{a b s} \mathcal{F} t=\mathcal{P}\left(1-e^{-\beta \cdot p}\right)
$$

where $\mathcal{F}$ is the flux and $\mathcal{P}$ is given by (8.43). Substituting the value of $\mathcal{P}$ from (8.46) we get

$$
\sigma_{a b s}=\frac{\mu^{2} \kappa_{5}^{2} L}{\mathcal{F}} \int d t d x(\mathcal{G}(t-i \epsilon, x)-\mathcal{G}(t+i \epsilon, x))
$$

In the above equation we have related the evaluation of the absorption crosssection to the evaluation of the thermal Green's function. Evaluating the integral one obtains

$$
\begin{aligned}
\sigma_{a b s}= & \frac{\mu^{2} \kappa_{5}^{2} L \mathcal{C}_{O}}{\mathcal{F}} \frac{\left(2 \pi T_{L}\right)^{2 h-1}\left(2 \pi T_{R}\right)^{2 \bar{h}-1}}{\Gamma(2 h) \Gamma(2 \bar{h})} \frac{e^{\beta \cdot p / 2}-(-1)^{2 h+2 \bar{h}} e^{-\beta \cdot p / 2}}{2} \\
& \left|\Gamma\left(h+i \frac{p_{+}}{2 \pi T_{L}}\right) \Gamma\left(\bar{h}+i \frac{p_{-}}{2 \pi T_{R}}\right)\right|^{2}
\end{aligned}
$$

where $\mathcal{C}_{O}$ is the coefficient of the leading order term in the OPE of the two-point function of operator $\mathcal{O}$.

\subsection{Absorption cross-section of minimal scalars from the D1-D5 SCFT}

In the previous section we related the thermal Green's function of the SCFT operator to the absorption cross-section. We will apply the results of the previous section for the case of the minimal scalars. We will consider the case of the minimal scalars corresponding to the fluctuation of the metric of $T^{4}$. Let the background metric of the torus be $\delta_{i j}$. Consider the minimal scalar $h_{67}$. We know the SCFT operator corresponding to this has conformal dimension $(1,1)$. From Section 8.4 we know that $\mu=1$. The interaction Lagrangian is given by

$$
S_{\text {int }}=2 T_{\text {eff }} \int d^{2} z h_{67} \partial x_{A}^{6}(z, \bar{z}) \bar{\partial} x_{A}^{7}(z, \bar{z})
$$

where we have set $\mu=1$. The factor of 2 arises because of the symmetric property of $h_{67} . S_{0}$ is given by

$$
S_{0}=T_{\text {eff }} \int d^{2} z \partial x_{A}^{i}(z, \bar{z}) \bar{\partial} x_{A}^{j}(z, \bar{z})
$$

Comparing with the previous section the operator $\mathcal{O}=2 T_{\text {eff }} \partial x_{A}^{6}(z, \bar{z}) \bar{\partial} x_{A}^{7}(z, \bar{z})$. For the absorption of a quanta of energy $\omega$ using (8.49) we obtain

$$
\sigma_{a b s}=2 \pi^{2} r_{1}^{2} r_{5}^{2} \frac{\pi \omega}{2} \frac{\exp \left(\omega / T_{H}\right)-1}{\left(\exp \left(\omega / 2 T_{R}\right)-1\right)\left(\exp \left(\omega / 2 T_{L}\right)-1\right)}
$$


where we have $L=2 \pi R_{5}, \mathcal{F}=\omega$, (8.42) for $\kappa_{5}$ and (8.37) for $\mathcal{C}_{O}$. Comparing the absorption cross-section of the minimal scalars obtained from supergravity in (3.22) with (8.52) we find that

$$
\sigma_{a b s}(\mathrm{SCFT})=\sigma_{a b s}(\text { Supergravity })
$$

Thus the SCFT calculation and the supergravity calculation of the absorption cross-section agree exactly. As we have mentioned earlier, this implies an exact agreement of decay rates between SCFT and the semiclassical calculation.

It is important to note that we have used the $\mathcal{N}=(4,4)$ SCFT realized as a free SCFT on the orbifold $\mathcal{M}$ as the background Lagrangian $S_{0}$. As we have said before, this SCFT is non-singular and therefore cannot correspond to the case of the D1-D5 system with no moduli. In Section 9.3.1 we have argued that the supergravity calculation of the absorption cross-section is independent of moduli. Therefore it makes sense to compare it with the SCFT result for the case with moduli turned on. In the next section we will show that the SCFT calculation is also independent of the moduli.

Before closing this section, we should mention the semiclassical calculation of decay rates from BTZ black holes and its comparison to CFT [156, 157, 158]. The greybody factor for BTZ black hole is connected with that for the D1-D5 black hole in [159, 160].

\subsubsection{Absorption cross-section for the blow up modes}

Another point worth mentioning is that the method followed above for the calculation of the absorption cross-section from the SCFT can be easily extended for the case of minimal scalars corresponding to the four blow up modes. These minimal scalars are listed in the last two lines of (6.16). They are the self-dual NS B-field and a linear combination of the Ramond-Ramond four form and the zero form. The operators for these scalars are the $Z_{2}$ twists in the SCFT. Absorption crosssection calculations for these scalars cannot be performed on the 'effective string' model based on the DBI action. The simple reason being that these operators are not present in the 'effective' string model. Thus the 'effective' string model does not capture all the degrees of freedom of the D1-D5 black hole.

\subsection{Fixed scalars}

Out of the 25 scalars mentioned earlier which form part of the spectrum of IIB supergravity on $T^{4}$, five become massive when further compactified on $A d S_{3} \times S^{3}$. There is an important additional scalar field which appears after this compactification: $h_{55}$. Let us remind ourselves the notation used for the coordinates: $A d S_{3}:\left(x_{0}, x_{5}, r\right), S^{3}:(\chi, \theta, \phi) ; T^{4}:\left(x_{6}, x_{7}, x_{8}, x_{9}\right) . \quad r, \chi, \theta, \phi$ are spherical polar coordinates for the directions $x_{1}, x_{2}, x_{3}, x_{4}$. In terms of the D-brane wrappings, 
the D5 branes are wrapped along the directions $x_{5}, x_{6}, x_{7}, x_{8}, x_{9}$ and D1 branes are aligned along $x_{5}$. The field $h_{55}$ is scalar in the sense that it is a scalar under the local Lorentz group $S O(3)$ of $S^{3}$.

In what follows we will specifically consider the three scalars $\phi_{10}, h_{i i}$ and $h_{55}$. The equations of motion of these fields in supergravity are coupled and have been discussed in detail in the literature [101, 103, 104, 105, 106]. It turns out that the six-dimensional dilaton $\phi_{6}=\phi_{10}-h_{i i} / 4$ which is a linear combination of $h_{i i}$ and $\phi_{10}$ remains massless; it is part of the twenty massless (minimal) scalars previously discussed. The two other linear combinations $\lambda$ and $\nu$ are defined as (see case (2) after Eq. (3.3))

$$
\begin{aligned}
& \lambda=\frac{h_{55}}{2}-\frac{\phi_{10}}{2}+\frac{h_{i i}}{8} \\
& \nu=\frac{h_{i i}}{8}
\end{aligned}
$$

$\lambda$ and $\nu$ satisfy coupled differential equations. They can be decoupled by the following position independent linear transformation 104$]^{22}$.

$$
\begin{aligned}
& \lambda=(\cos \alpha) \phi_{+}+(\sin \alpha) \phi_{-} \\
& \nu=-(\sin \alpha) \phi_{+}+(\cos \alpha) \phi_{-}
\end{aligned}
$$

where $\alpha$ can be found by solving the equation

$$
\tan \alpha-\frac{1}{\tan \alpha}=\frac{2}{\sqrt{3}} \frac{Q_{1}+v Q_{5}}{Q_{1}-v Q_{5}}
$$

Then $\phi_{ \pm}$obey the following equations

$$
\left[\frac{1}{r^{3}} \partial r^{3} \partial_{r}+\omega^{2} f_{1} f_{5}-8 \frac{Q_{ \pm}^{2}}{r^{2}\left(r^{2}+Q_{ \pm}^{2}\right)^{2}}\right] \phi_{ \pm}=0
$$

where $\omega$ is the frequency of wave and

$$
Q_{ \pm}=\frac{\alpha^{\prime} g_{s}}{3}\left(Q_{5}+\frac{Q_{1}}{v} \mp \sqrt{Q_{5}^{2}+\left(\frac{Q_{1}}{v}\right)^{2}-\frac{Q_{5} Q_{1}}{v}}\right)
$$

These are examples of fixed scalars. The pick up masses in the background geometry of the D1-D5 system. To see this take the near horizon limit defined in (2.69) in the equation (8.57), we get (see also 161)

$$
\left[\frac{1}{l^{2} U} \frac{\partial}{\partial U}\left(U^{3} \frac{\partial}{\partial U}\right)+\frac{\omega^{2} l^{2}}{U^{2}}-\frac{8}{l^{2}}\right] \phi_{ \pm}=0
$$

\footnotetext{
${ }^{22}$ We have set $r_{0}=0$ in the equations in [104 as we are looking at the background corresponding to the D1-D5 system.
} 
Note that this is the Klein-Gordan equation of a massive scalar in $A d S_{3}$ with (mass) $^{2}=8$ is units of the $A d S_{3}$ units. Further more since the equation for both $\phi_{+}$and $\phi_{-}$is identical in the near horizon limit the equation of motions of $\lambda$ and $\nu$ become decoupled. They obey the massive Klein-Gordon equation in $A d S_{3}$. The near horizon mass of $\lambda$ and $\nu$ is $m^{2}=8$ in units of the radius of $A d S_{3}$.

Understanding the absorption and emission properties of fixed scalars is an important problem, because the D-brane computation and semiclassical black hole calculation of these properties are at variance [103, 104. The discrepancy essentially originates from the 'expected' couplings of $\lambda$ and $\nu$ to SCFT operators with $(h, \bar{h})=(1,3)$ and $(3,1)$ (see also 101). These SCFT operators lead to qualitatively different graybody factors from what the fixed scalars exhibit semiclassically. The semiclassical graybody factors are in agreement with D-brane computations if the couplings were only to $(2,2)$ operators.

The coupling to $(1,3)$ and $(3,1)$ operators is guessed from qualitative reasoning based on the Dirac-Born-Infeld action. Since we now have a method of deducing the couplings to the bulk fieds based on near-horizon symmetries, let us use it in the case of the fixed scalars.

(a) By the mass dimension relation (6.14) we see that the fixed scalars $\lambda$ and $\nu$ correspond to operators with weights $h+\bar{h}=4$.

(b) The fixed scalars have $S U(2)_{E} \times \widetilde{S U(2)_{E}}$ quantum numbers $(\mathbf{1}, \mathbf{1})$.

As all the supergravity fields are classified according to the short multiplets of $S U(1,1 \mid 2) \times S U(1,1 \mid 2)$ we can find the field corresponding to these quantum numbers among the short multiplets. Searching through the short multiplets (see below (6.12)), we find that the fixed scalars belong to the short multiplet $(3,3)_{S}$ of $S U(1,1 \mid 2) \times S U(1,1 \mid 2)$. They occur as top component of $(\mathbf{3}, \mathbf{3})_{S}$. There are six fixed scalars in all. We conclude that the operators with $(h, \bar{h})=(1,3)$ or $(h, \bar{h})=(3,1)$ (which were inferred by the DBI method) are ruled out by the analysis of symmetries.

In summary, since the $(1,3)$ and $(3,1)$ operators are ruled out by our analysis, the discrepancy between the D-brane calculation and the semiclassical calculation of absorption and emission rates disappears. Using the coupling to $(2,2)$ operators as we derived above, we can compute $\sigma_{a b s}$ for fixed scalars using (8.49). This agrees exactly with the result (3.27).

\subsection{Intermediate Scalars}

We only make the remark that the classification presented in Section 6.1 correctly accounts for all sixteen intermediate scalars, and predict that they should couple to SCFT operators with $(h, \bar{h})=(1,2)$ belonging to the short multiplet $(\mathbf{2}, \mathbf{3})_{S}$ or operators with $(h, \bar{h})=(2,1)$ belonging to the short multiplet $(\mathbf{3}, \mathbf{2})_{S}$ (see below equation (6.12)). This agrees with the 'phenomenological' prediction made earlier in the literature [162]. 


\section{Non-renormalization theorems}

Let us review the three major agreements between the $\mathcal{N}=(4,4) \operatorname{SCFT}$ on $\mathcal{M}$ and supergravity. We showed in section 6 that the spectrum of short multiplets of $\mathcal{N}=$ $(4,4) \mathrm{SCFT}$ on $\mathcal{M}$ was in one to one correspondence with the supergravity modes in the near horizon geometry of the D1-D5 system. In sections 5 and 8 we saw that the entropy calculated from the microscopic SCFT agreed with that of the D1-D5 black hole. Finally in section 8 we have seen that the calculation of Hawking radiation from the SCFT agreed precisely with the semiclassical calculation including the gray body factors.

In this section we will discuss the validity of these calculations both in the boundary SCFT and the bulk supergravity. The validity of the calculations performed on the conformal field theory side in general do not overlap with that on the supergravity. Conformal field theory calculations are performed using the $\mathcal{N}=(4,4)$ free orbifold theory on $\mathcal{M}$. In section 7 we saw that the SCFT corresponding to supergravity is singular and presumably involves a large deformation in the moduli space from the free orbifold conformal field theory. Therefore the calculations performed in supergravity are valid in the region of moduli space when the conformal field theory is singular.

Let us compare the moduli space of deformations on the SCFT and the supergravity. The supergravity and the SCFT moduli are listed in (6.16). They are in one to one correspondence. There are 20 moduli in all. On the supergravity side the moduli parameterize the homogeneous space $\widetilde{\mathcal{M}}=S O(4,5) / S O(4) \times S O(5))$ [139, 114, 119]. On the SCFT $\mathcal{N}=(4,4)$ supersymmetry highly constraints the metric on the moduli space to also be $\widetilde{\mathcal{M}}[163$. Therefore for every point on the moduli space of the SCFT there is a corresponding point on the SCFT. As we have seen in section 7 the D1-D5 supergravity solution and the free orbifold SCFT are at different points in the moduli space. Therefore it is natural to conclude that there are non-renormalization theorems that allow us to interpolate between the calculations done using the free orbifold theory and supergravity calculations. In this section we will detail these non-renormalization theorems for the three calculations, the spectrum of short multiplets, the entropy and Hawking radiation.

\subsection{The spectrum of short multiplets}

Short multiplets in the SCFT are built on chiral primaries both for the left and right movers (see section 5.5). The chiral primaries satisfy a BPS bound. Their R-charge is the same as the conformal dimension. Thus their spectrum in independent of any perturbation of the conformal field theory which preserves the $\mathcal{N}=(4,4)$ supersymmetric structure [132, 164] The entire structure of short multiplets is then dictated by the $\mathcal{N}=(4,4)$ SCFT algebra. Therefore the spectrum of short multiplets is invariant under deformations of the SCFT. In section 5.8 
the entire set of short multiplets was evaluated using the free orbifold theory on $\mathcal{M}$. This will remain invariant under deformations of this SCFT from the free orbifold point. A further piece of evidence that the shormultiplets structure does not change under derformations is that the number of chiral primaries which form the bottom component of a short multiplet can be counted in the case of SCFT on $\mathcal{M}$ using a topological partition function [165]. This partition function is invariant under deformation of the SCFT.

The short multiplet structure of the supergravity modes obtained in section 6.1 ignored the winding and momentum modes on the torus. In fact the mass spectrum would not change if there were any metric deformation or Neveu-Schwarz $B$-field through the torus. These affect only winding and momentum modes on the torus. Thus the short multiplet structure of the supergravity modes presented in (6.12) are invariant under deformations of the moduli that involve the traceless components of the metric and the self-dual NS B-field (6.16).

We have just demonstrated that calculation of the spectrum of short multiplets in the supergravity and the SCFT is independent of the moduli This allows us to compare the short multiplet spectrum on both sides and obtain agreement.

\subsection{Entropy and area}

As we have seen in section 8 the black hole is represented as a state with $L_{0} \neq$ $0, \bar{L}_{0} \neq 0$ over the Ramond sector of the SCFT. The entropy in the SCFT is calculated by evaluating the asymptotic density of states of with these values of $L_{0}$ and $\bar{L}_{0}$. The asymptotic density of states in a conformal field theory is given by Cardy's formula [115]. This depends on the level and the central charge of the conformal field theory. The central charge of a conformal field theory is independent of moduli. The Entropy is given by the logarithm for the asymptotic density of states. Therefore the entropy calculated from the SCFT remains invariant for various values of moduli.

The Bekenstein-Hawking entropy in supergravity is evaluated in the five-dimensional Einstein metric and is equal to the area of the Horizon. From the equations of motion of type IIB supergravity [101], we can explicitly see that the five dimensional Einstein metric is not changed by turning on the sixteen moduli listed on the top three lines of (6.16). These are the traceless components of the metric, the selfdual NS $B$-field along the torus and the six-dimensional dilaton. This can also be seen explicitly form the supergravity solution with moduli (2.48) constructed in 88. Thus the area of the D1-D5 black hole does not change with moduli in supergravity. This ensures that the evaluation of the entropy from SCFT will agree with that in supergravity even though they are evaluated at different points in the moduli space. 


\subsection{Hawking radiation}

Before we discuss the dependence of Hawking radiation on the moduli let us examine the various approximations made in the derivation of Hawking radiation both in supergravity and in SCFT. Recall that the semi-classical calculations of Hawking radiation was done in the dilute gas limit (3.9) $r_{0}, r_{n} \ll r_{1}, r_{5}$. We also made the approximation of low energies compared to the horizon radius $\omega r_{5} \ll 1$. As the SCFT calculation relies very much on the near horizon limit and the enhanced symmetries near the horizon, one can ask the question whether in the near horizon limit the dilute gas approximation is obeyed. Let us convert $r_{0}, r_{n}, r_{1}, r_{5}$ to near horizon variables.

$$
\begin{gathered}
r_{0}=U_{0} \alpha^{\prime} \quad r_{n}=U_{0} \sinh \sigma \alpha^{\prime} \\
r_{1}=\sqrt{\frac{g_{s} Q}{v} \alpha^{\prime}} \quad r_{5}=\sqrt{g_{s} Q_{5} \alpha^{\prime}}
\end{gathered}
$$

Now it is easy to see in the near horizon limit $\alpha^{\prime} \rightarrow 0$ the dilute gas approximation always holds.

We also use the low energy approximation in the SCFT calculation. In the microscopic calculations of Hawking radiation from the SCFT we restricted our attention to first order in perturbation theory. In fact we used the Fermi-golden rule to obtain the absorption cross-section in (8.9). It is easy to see that for the metric fluctuation the higher order terms in perturbation theory go in powers of $w^{2} r_{5}^{2}$ [166]. Thus higher order terms in the SCFT calculation are suppressed due to the low energy approximation.

In spite of the fact that the dilute gas approximation and the low energy approximation are made on both the supergravity and SCFT side the Hawking radiation calculation from the D1-D5 black hole in supergravity and the SCFT are done at different point in the moduli space. We now discuss why in spite of this they both agree.

\subsubsection{Independence of Hawking radiation calculation on moduli: Su- pergravity}

We recall that the D1-D5 black hole solution in the absence of moduli is 71 , 44] obtained from the D1-D5 system by further compactifying $x^{5}$ on a circle of radius $R_{5}$ and adding left(right) moving Kaluza-Klein momenta along $x^{5}$. The corresponding supergravity solution is given

The absorption cross-section of minimal scalars in the absence of moduli is given by (3.22) [38, 40]. We will now show that the absorption cross-section remains unchanged even when the moduli are turned on.

From the equations of motion of type IIB supergravity [101, we can explicitly

see that the five-dimensional Einstein metric $d s_{5, \text { Ein }}^{2}$ is not changed by turning on 
the sixteen moduli corresponding to the metric $G_{i j}$ on $T^{4}$ and the Ramond-Ramond 2 -form potential $B$. As regards the four blowing up moduli, the invariance of $d s_{5, \text { Ein }}^{2}$ can be seen from the fact that turning on these moduli corresponds to $S O(4,5)$ transformation (which is a part of a U-duality transformation) and from the fact that the Einstein metric does not change under U-duality. Now we know that the minimal scalars $\phi^{i}$ all satisfy the wave-equation

$$
D_{\mu} \partial^{\mu} \phi^{i}=0
$$

where the Laplacian is with respect to the Einstein metric in five dimensions. Since it is only this wave equation that determines the absorption cross-section completely, we see that $\sigma_{a b s}$ is the same as before.

It is straightforward to see that the Hawking rate, given by (3.25) is also not changed when moduli are turned on.

\subsubsection{Independence of Hawking radiation on moduli:SCFT}

In this section we will study the independence of the Hawking radiation on D1-D5 moduli. In Section 2 we have listed the twenty $(1,1)$ operators $\mathcal{O}_{i}(z, \bar{z})$ in the SCFT based on the symmetric product orbifold $\mathcal{M}$ which is dual to the D1-D5 system. Turning on various moduli $\phi^{i}$ of supergravity corresponds to perturbing the SCFT

$$
S=S_{0}+\sum_{i} \int d^{2} z \bar{\phi}^{i} \mathcal{O}_{i}(z, \bar{z})
$$

where $\bar{\phi}^{i}$ denote the near-horizon limits of the various moduli fields $\phi^{i}$. We note here that $S_{0}$ corresponds to the free SCFT based on the symmetric product orbifold $\mathcal{M}$. As we have seen in section 7 that this SCFT is non-singular (all correlation functions are finite), it does not correspond to the marginally stable BPS solution originally found in [71, 44 Instead, it corresponds to a five-dimensional black hole solution in supergravity with suitable "blow-up" moduli turned on.

Let us now calculate the absorption cross-section of a supergravity fluctuation $\delta \phi_{i}$ to the thermal Green's function of the corresponding operator of the SCFT. The notation $\delta \phi_{i}$ implies that we are considering the supergravity field to be of the form

$$
\phi^{i}=\phi_{0}^{i}+\mu \delta \phi^{i}
$$

where $\phi_{0}^{i}$ represents the background value and $\mu$ is the strength of the coupling.

$$
\begin{aligned}
S & =S_{0}+\int d^{2} z\left[\bar{\phi}_{0}^{i}+\mu \delta \bar{\phi}^{i}\right] \mathcal{O}_{i}(z, \bar{z}) \\
& =S_{\phi_{0}}+S_{i n t}
\end{aligned}
$$

where

$$
S_{\phi_{0}}=S_{0}+\int d^{2} z \bar{\phi}_{0}^{i} \mathcal{O}_{i}(z, \bar{z})
$$




$$
S_{\text {int }}=\mu \int d^{2} z \delta \bar{\phi}^{i} \mathcal{O}_{i}(z, \bar{z})
$$

As we have seen in the Section 8.5 the absorption cross-section of the supergravity fluctuation $\delta \phi^{i}$ involves essentially the two-point function of the operator $\mathcal{O}_{i}$ calculated with respect to the SCFT action $S_{\phi_{0}}$. Since $\mathcal{O}_{i}$ is a marginal operator, its two-point function is completely determined apart from a constant. Regarding the marginality of the operators $\mathcal{O}_{i}$, it is easy to establish it upto one-loop order by direct computation $\left(c_{i j k}=0\right)$. The fact that these operators are exactly marginal can be argued as follows. The twenty operators $\mathcal{O}_{i}$ arise as top components of five chiral primaries. It is known that the number of chiral primaries with $\left(j_{R}, \tilde{j}_{R}\right)=(m, n)$ is the Hodge number $h_{2 m, 2 n}$ of the target space $\mathcal{M}$ of the SCFT. Since this number is a topological invariant, it should be the same at all points of the moduli space of deformations.

We showed in Section 8.4 that if the operator $\mathcal{O}_{i}$ corresponding to $h_{i j}$ is canonically normalized (OPE has residue 1 ) and if $\delta \phi_{i}$ is canonically normalized in supergravity, then the normalization of $S_{\text {int }}$ as in (9.7) ensures that $\sigma_{a b s}$ from SCFT agrees with the supergravity result. The crucial point now is the following: once we fix the normalization of $S_{\text {int }}$ at a given point in moduli space, at some other point it may acquire a constant $(\neq 1)$ in front of the integral when $\mathcal{O}_{i}$ and $\delta \phi_{i}$ are canonically normalized at the new point. This would imply that $\sigma_{a b s}$ will get multiplied by this constant, in turn implying disagreement with supergravity. We need to show that this does not happen.

To start with a simple example, let us first restrict to the moduli $g_{i j}$ of the torus $\widetilde{T^{4}}$. We have

$$
S=\int d^{2} z \partial x^{i} \bar{\partial} x^{j} g_{i j}
$$

The factor of string tension has been absorbed in the definition of $x^{i}$.

In Section 8.4 we had $g_{i j}=\delta_{i j}+h_{i j}$, leading to

$$
\begin{aligned}
S & =S_{0}+S_{i n t} \\
S_{0} & =\int d^{2} z \partial x^{i} \bar{\partial} x^{j} \delta_{i j} \\
S_{i n t} & =\int d^{2} z \partial x^{i} \bar{\partial} x^{j} h_{i j}
\end{aligned}
$$

In the above equation we have set $\mu=1$. As we have remarked above, this $S_{\text {int }}$ gives rise to the correctly normalized $\sigma_{a b s}$.

Now, if we expand around some other metric

$$
g_{i j}=g_{0 i j}+h_{i j}
$$

then the above action $(9.8)$ implies

$$
S=S_{g_{0}}+S_{\text {int }}
$$




$$
\begin{aligned}
S_{g_{0}} & =\int d^{2} z \partial x^{i} \bar{\partial} x^{j} g_{0 i j} \\
S_{\text {int }} & =\int d^{2} z \partial x^{i} \bar{\partial} x^{j} h_{i j}
\end{aligned}
$$

Now the point is that neither $h_{i j}$ nor the operator $\mathcal{O}^{i j}=\partial X^{i} \bar{\partial} X^{j}$ in $S_{\text {int }}$ is canonically normalized at $g_{i j}=g_{0 i j}$. When we do use the canonically normalized operators, do we pick up an additional constant in front?

Note that

$$
\left\langle\mathcal{O}^{i j} \mathcal{O}^{k l}\right\rangle_{g_{0}}=g_{0}^{i k} g_{0}^{j l}|z-w|^{-4}
$$

and

$$
\left\langle h_{i j}(x) h_{k l}(y)\right\rangle_{g_{0}}=g_{0, i k} g_{0, j l} \mathcal{D}(x, y)
$$

where $\mathcal{D}(x, y)$ is the massless scalar propagator

This shows that

Statement (1): The two-point functions of $\mathcal{O}^{i j}$ and $h_{i j}$ pick up inverse factors

As a result, $S_{\text {int }}$ remains correctly normalized when re-written in terms of the canonically normalized $h$ and $\mathcal{O}$ and no additional constant is picked up.

The above result is in fact valid in the full twenty dimensional moduli space $\widetilde{\mathcal{M}}$ because Statement (1) above remains true generally.

To see this, let us first rephrase our result for the special case of the metric moduli (9.8) in a more geometric way. The $g_{i j}$ 's can be regarded as some of the coordinates of the moduli space $\widetilde{\mathcal{M}}$ (known to be a coset $S O(4,5) /(S O(4) \times$ $S O(5)))$. The infinitesimal perturbations $h_{i j}, h_{k l}$ can be thought of as defining tangent vectors at the point $g_{0, i j}$ (namely the vectors $\partial / \partial g_{i j}, \partial / \partial g_{k l}$ ). The (residue of the) two-point function given by (9.12) defines the inner product between these two tangent vectors according to the Zamolodchikov metric [167, 163].

The fact that the moduli space $\widetilde{\mathcal{M}}$ of the $\mathcal{N}=(4,4)$ SCFT on $\mathcal{M}$ is the coset $S O(4,5) /(S O(4) \times S O(5))$ is argued in 163. If the superconformal theory has $\mathcal{N}=(4,4)$ supersymmetry and if the dimension of the moduli space is $d$ then it is shown in 163 that the moduli space of the symmetric the product SCFT is given by

$$
\frac{S O(4, d / 4)}{S O(4) \times S O(d / 4)}
$$

As a simple check note that the dimension of the space is (9.14) is $d$. The outline of the argument is a follows. An $\mathcal{N}=(4,4)$ SCFT has superconformal $S U(2)_{R} \times$ $\widetilde{S U(2)_{R}}$ symmetry. We have seen that the bottom component of the short multiplet which contains the marginal operator $(\mathbf{2}, \mathbf{2})_{\mathrm{S}}$ transforms as a $(\mathbf{2}, \mathbf{2})$ under $S U(2)_{R} \times$ $S \widetilde{U(2)}{ }_{R}$. The top component which corresponds to the moduli transforms as a $(\mathbf{1}, \mathbf{1})$ under the R-symmetry. The holonomy group of the Zamolodchikov metric 
should leave invariant the action of $\left.S U(2)_{R} \times S \widetilde{S(2}\right)_{R}$. Then the holonomy group should have a form

$$
K \subset S U(2) \times S U(2) \times \tilde{K} \subset S O(d)
$$

Then (9.15) together with $\mathcal{N}=(4,4)$ supersymmetry and the left-right symmetry of the two $S U(2)_{R}$ 's of the SCFT fixes the moduli space to be uniquely that given in (9.14). We have found in Section 2 that there are 20 marginal operators for the $\mathcal{N}=(4,4) \mathrm{SCFT}$ on the orbifold $\mathcal{M}$. Therefore the dimension of the moduli space is 20. Thus $\widetilde{\mathcal{M}}$ is given by

$$
\widetilde{\mathcal{M}}=\frac{S O(4,5)}{S O(4) \times S O(5)}
$$

Consider, on the other hand, the propagator (inverse two-point function) of $h_{i j}, h_{k l}$ in supergravity. The moduli space action of low energy fluctuations is nothing but the supergravity action evaluated around the classical solutions $g_{0, i j}$. The kinetic term of such a moduli space action defines the metric of moduli space. The statement (1) above is a simple reflection of the fact that the Zamolodchikov metric defines the metric on moduli space, and hence

Statement (2): The propagator of supergravity fluctuations, viewed as a matrix, is the inverse of the two-point functions in the SCFT.

The last statement is of course not specific to the moduli $g_{i j}$ and is true of all the moduli. We find, therefore, that fixing the normalization of $S_{\text {int }}$ (9.7) at any one point $\phi_{0}$ ensures that the normalization remains correct at any other point $\phi_{0}^{\prime}$ by virtue of Statement (2). We should note in passing that Statement (2) is consistent with, and could have been derived from AdS/CFT correspondence as applied to the two-point function.

Thus, we find that $\sigma_{a b s}$ is independent of the moduli, in agreement with the result from supergravity. 


\section{Strings in $A d S_{3}$}

The AdS/CFT correspondence [42, 62, 63, 90, 41] for the case of the D1-D5 system states that type IIB string theory on $A d S_{3} \times S^{3} \times T^{4}$ is dual to the $1+1$ dimensional conformal field theory of the Higgs branch of the gauge theory of the D1-D5 system. In our study of the bulk geometry we have worked only in the supergravity approximation of string theory on $A d S_{3} \times S^{3}$. To fully explore the AdS/CFT correspondence for the D1-D5 system we need to understand string propagation in the bulk geometry. String theory on $A d S_{3} \times S^{3} \times T^{4}$ involves Ramond-Ramond fluxes through the $S^{3}$. Progress in formulation of string propagation in Ramond-Ramond backgrounds for the case of $A d S_{3}$ have been made in [168, 169, 170, 171, 172, 173] Unfortunately, these models have been difficult to quantize and results have been hard to obtain. It is more convenient to study string theory on the background which is S-dual to the D1-D5 system. The near horizon geometry of the S-dual is also $A d S_{3} \times S^{3} \times T^{4}$ but with Neveu-Schwarz H-fluxes through the $S^{3}$ and $A d S_{3}$. String propagation on this dual background can be quantized. It also provides an exact string background in which the metric $g_{00}$ is non-trivial. An important result from the study of strings in $A d S_{3}$ has been in understanding the role of long strings in the spectrum. We saw in section 7 that there exists long D1 brane solutions near the boundary of $A d S_{3}$. Therefore, in the S-dual geometry this would mean that there exists long fundamental strings. These strings have been constructed as classical solutions and also have been identified in the full quantum spectrum [174, 175]. They play an important role in constructing a consistent spectrum of strings in $\mathrm{AdS}_{3}$.

In this section 10.1 we will introduce the S-dual of the D1-D5 system. We then formulate string propagation on $A d S_{3}$ and study its spectrum in section 10.2. Our discussion will be based on [174. To understand the essential aspects of the spectrum it is enough to focus on the $A d S_{3}$ part of the geometry, we also restrict our discussion to bosonic strings in $A d S_{3}$. In section 10.3 briefly review string propagation in Euclidean $A d S_{3}$ which was initiated in [139]. We then write down the long string solution in Euclidean $A d S_{3}$ and discuss its symmetries [114. Finally in section 10.4 we discuss string theory on thermal $A d S_{3}$ backgrounds. The one loop free energy of a gas of strings in $A d S_{3}$ was evaluated in 175. It was shown to be modular invariant and the space-time spectrum read off from it matched with the proposal for the spectrum in [174].

We just mention two topics which we will not have the time to review. For a discussion of correlation functions for string theory on $A d S_{3}$ and their role in the AdS/CFT correspondence see [176] and references there in. Branes in $A d S_{3}$ has been extensively studied for a recent work see [177, 178] and references there in. 


\subsection{The S-dual of the D1-D5 system}

Let us consider the S-dual of the D1-D5 whose metric is given in (2.24). S-duality takes $\phi \rightarrow-\phi, C^{2} \rightarrow B_{N S}$ and $d s^{2} \rightarrow e^{-\phi} d s^{2}$. Performing these operations on the supergravity solution given in (2.24) along with a rescaling of the coordinates by $\sqrt{g_{s}}$ (coordinates are rescaled to keep the 10-dimensional Newton's constant invariant) we obtain

$$
\begin{aligned}
d s^{2} & =f_{1}^{-1}\left(-d t^{2}+d x_{5}^{2}\right)+f_{5}\left(d x_{1}^{2}+\cdots+d x_{4}^{2}\right) \\
& +\left(d x_{6}^{2}+\cdots+d x_{9}^{2}\right) \\
e^{-2 \phi} & =\frac{1}{g_{s}^{\prime 2}} f_{1} f_{5}^{-1}, \\
B_{05} & =\frac{1}{2}\left(f_{1}^{-1}-1\right), \\
H_{a b c} & =\frac{1}{2} \epsilon_{a b c d} \partial_{d} f_{5}, \quad a, b, c, d=1,2,3,4
\end{aligned}
$$

where $g_{s}^{\prime}=1 / g_{s}$ and

$$
f_{1}=1+\frac{16 \pi^{4} g_{s}^{\prime 2} \alpha^{\prime 3} Q_{1}}{V_{4}^{\prime} r^{2}}, \quad f_{5}=1+\frac{\alpha^{\prime} Q_{5}}{r^{2}} .
$$

Here $V_{4}^{\prime}$ refers to the volume of the $T^{4}$ measured in the scaled coordinates. From the Neveu-Schwarz fluxes it is now easy to see that this system is the supergravity solution of $Q_{5}$ Neveu-Schwarz branes with $Q_{1}$ fundamental strings smeared over the four torus $T^{4}$.

Let us now take the near horizon limit of this solution. This is given by

$$
\begin{array}{r}
\alpha^{\prime} \rightarrow 0, \quad \frac{r}{\alpha^{\prime}} \equiv U=\text { fixed } \\
v^{\prime} \equiv \frac{V_{4}^{\prime}}{16 \pi^{4} \alpha^{\prime 2}}=\text { fixed }, \quad g_{6}^{\prime} \equiv \frac{g_{s}^{\prime}}{\sqrt{v}^{\prime}}=\text { fixed }
\end{array}
$$

Under this scaling limit the metric given in (10.1) reduces to

$$
\begin{aligned}
d s^{2} & =\alpha^{\prime} U^{2} Q_{5}\left(-d t^{2}+d x_{5}^{2}\right)+\alpha^{\prime} Q_{5} \frac{d U^{2}}{U^{2}}+\alpha^{\prime} Q_{5} d \Omega^{2} \\
& +\left(d x_{6}^{2}+\cdots d x_{9}^{2}\right), \\
e^{-2 \phi} & =\frac{Q_{1}}{v^{\prime} Q_{5}}, \\
H_{05 U} & =\alpha^{\prime} Q_{5} U, \\
H_{\theta \phi \chi} & =\alpha^{\prime} Q_{5},
\end{aligned}
$$

Here we have rescaled coordinates $t$ and $x_{5}$ by $\sqrt{Q_{1} Q_{5} g_{6}^{\prime 2}}$. Thus the near horizon geometry of $Q_{1}$ fundamental strings and $Q_{5}$ NS branes is $A d S_{3} \times S^{3} \times T^{4}$ with NeveuSchwarz fluxes. The radius of $S^{3}$ is $\sqrt{Q_{5} \alpha^{\prime}}$. Note that the near horizon geometry 
depends on $Q_{1}$ only through the string coupling constant which is proportional to the ratio $Q_{1} / Q_{5}$.

It is convenient to study string propagation on this geometry as it consists only of Neveu-Schwarz fluxes. We will restrict out attention to string propagation only on the $A d S_{3}$ part of the geometry. Strings on $S^{3}$ with $H$ flux through the sphere is an $S U(2)$ WZW model at level $Q_{5}$, while strings on $T^{4}$ is a free field conformal theory. We refer to these conformal field theories as the internal conformal field theory. To simplify the discussion we will study only bosonic strings on $A d S_{3}$.

\subsection{String propagation on $A d S_{3}$}

String propagation on $A d S_{3}$ with $H$ flux is an exact conformal field theory $A d S_{3}$ is a $S L(2, R)$ group manifold. To see this consider the $S L(2, R)$ group element parameterized by

$$
g=\exp \left(i \frac{t+\phi}{2} \sigma_{2}\right) \exp \left(\rho \sigma_{3}\right) \exp \left(i \frac{t-\phi}{2} \sigma_{2}\right),
$$

where $\sigma_{i}$ are the Pauli matrices. We use the following generators for the $S L(2, R)$ Lie algebra

$$
T^{3}=-\frac{i}{2} \sigma^{2}, \quad T^{ \pm}=\frac{1}{2}\left(\sigma^{3} \pm i \sigma_{1}\right) .
$$

Then the metric on the $S L(2, R)$ group manifold is given by

$$
g_{\mu \nu}=\frac{1}{2} \operatorname{Tr}\left(g^{-1} \partial_{\mu} g g^{-1} \partial_{\nu} g\right)
$$

where $\mu, \nu$ are indices referring to $\rho, t, \phi$. Evaluating the metric using the parameterization given in (10.5) we get

$$
d s^{2}=-\cosh ^{2} \rho d t^{2}+d \rho^{2}+\sinh ^{2} \rho d \phi^{2},
$$

which is the metric on $A d S_{3}$ expressed in the global coordinates $(t, \phi, \rho)$ (C.6). Thus string propagation on $A d S_{3}$ can be expressed in terms of the WZW action given below

$$
S=\frac{Q_{5}}{4 \pi \alpha^{\prime}} \int_{M} d x^{+} d x^{-} \operatorname{Tr}\left(g^{-1} \partial^{+} g g^{-1} \partial^{-} g\right)+\frac{Q_{5}}{12 \pi \alpha^{\prime}} \int_{N} \operatorname{Tr}\left(\omega^{3}\right) .
$$

Here $M$ is the embedding of the world sheet into the group manifold $A d S_{3}$ and $N$ is any 3 dimensional manifold whose boundary is $M$ and $\omega=g^{-} d g$ the MaurerCartan 1-form. The second term is called the Wess-Zumino term. We have used Minkowski signature on the world sheet and $x^{ \pm}=\tau \pm \sigma$, where $\tau$ and $\sigma$ are the world sheet time and position coordinate. As a check on the action compare the $H$ field in (10.4) and the one induced by the Wess-Zumino term. It is easily seen that both are proportional to the volume form on $\mathrm{AdS}_{3}$. 


\section{Classical solutions}

Now that we have the action of strings in $A d S_{3}$ we obtain the form of the classical solutions. The equations of motion derived form the action in (10.9) is given by

$$
\partial^{-}\left(\partial^{+} g g^{-1}\right)=0 .
$$

Thus a general solution of this action is given by

$$
g=g_{+}\left(x^{+}\right) g_{-}\left(x^{-}\right)
$$

It is thus easy to construct classical solutions. Consider the following solution

$$
g_{+}\left(x^{+}\right)=e^{\frac{i \alpha \sigma^{2}}{2} x^{+}} \quad g_{-}\left(x^{-}\right)=e^{\frac{i \alpha \sigma^{2}}{2} x^{-}}
$$

For this solution we have $g=e^{-\alpha \tau \sigma^{2}}$. From the parametrization of the group element in (10.5) we see that the solution is a timelike geodesic with $\rho=0, \phi=0$ and $t=\alpha \tau$. Note that the solution does not have $\sigma$ dependence, therefore it represents a particle trajectory. Space like geodesics can also be constucted. The solution

$$
g_{+}\left(x^{+}\right)=e^{\frac{\alpha \sigma^{3}}{2} x^{+}} \quad g_{-}\left(x^{-}\right)=e^{\frac{\alpha \sigma^{3}}{2} x^{-}}
$$

represents a spacelike geodesic with $g=e^{\alpha \tau \sigma^{3}}$. From (10.5) we see that the trajectory is given by $\rho=\alpha \tau$ which is space like.

It is interesting to note that there is a symmetry which allows the generation of new solutions given one solution. The transformation

$$
g_{+}=e^{i \frac{1}{2} \omega x^{+} \sigma_{2}} \tilde{g}_{+} \quad g_{-}=e^{i \frac{1}{2} \omega x^{-} \sigma_{2}} \tilde{g}_{+}
$$

where $\tilde{g}_{+}$and $\tilde{g}_{-}$are the old solution is also a solution. From (10.5) we see that this acts on $t$ and $\phi$ as

$$
t \rightarrow t+\omega \tau \quad \phi \rightarrow \phi+\omega \sigma
$$

The periodicity of the string worldsheet under $\sigma \rightarrow \sigma+2 \pi$ is obeyed. This transformation is called spectral flow. It stretches the geodesic in the $t$-direction gives $\sigma$ dependence to the geodesics. In fact now the solution represents a string winding $w$ times around the centre $\rho=0$ of $A d S_{3}$. The spectral flow of timelike geodesics are called short strings, their energy is bounded from above. While the spectral flow of spacelike geodescis are called long strings, their energy is bounded from below. Thus long strings are like scattering states while short strings are like bound states in $A d S_{3}$ [174]. These long strings as we will see in section 10.4 are the duals of the long D-strings discussed in section 7.

Symmetries of the $S L(2, R)$ WZW action

The WZW action has an infinite set of conserved charges given by

$$
J_{n}^{a}=Q_{5} \int_{0}^{2 \pi} \frac{d x^{+}}{2 \pi} e^{i n x^{+}} \operatorname{Tr}\left(T^{a} \partial_{+} g g^{-1}\right) \quad \bar{J}_{n}^{a}=Q_{5} \int_{0}^{2 \pi} \frac{d x^{-}}{2 \pi} e^{i n x^{-}} \operatorname{Tr}\left(T^{a} \bar{\partial}_{-} g g^{-1}\right)
$$


They obey the commutation relations

$$
\begin{aligned}
{\left[J_{n}^{3}, J_{m}^{3}\right] } & =-\frac{Q_{5}}{2} n \delta_{n+m, 0}, \\
{\left[J_{n}^{3}, J_{m}^{ \pm}\right] } & = \pm J_{n+m}^{ \pm} \\
{\left[J_{n}^{+}, J_{m}^{-}\right] } & =-2 J_{n+m}^{3}+Q_{5} n \delta_{n+m, 0} .
\end{aligned}
$$

There is a similar set of commutation relations for the right movers $\bar{J}_{n}^{a}$. Using the Sugawara construction one can define the Virasoro generators, they are given by

$$
\begin{aligned}
L_{0}= & \frac{1}{Q_{5}-2}\left[\frac{1}{2}\left(J_{0}^{+} J_{0}^{-}+J_{0}^{-} J_{0}^{+}\right)-\left(J_{0}^{3}\right)^{2}\right. \\
& \left.+\sum_{m=1}^{\infty}\left(J_{-m}^{+} J_{m}^{-}+J_{-m}^{-} J_{m}^{+}-2 J_{-m}^{3} J_{m}^{3}\right)\right] \\
L_{n \neq 0}= & \frac{1}{Q_{5}-2} \sum_{m=1}^{\infty}\left(J_{n-m}^{+} J_{m}^{-}+J_{n-m}^{-} J_{m}^{+}-2 J_{n-m}^{3} J_{m}^{3}\right)
\end{aligned}
$$

These generators obey the Virasoro algebra with the central charge given by

$$
c=\frac{3 Q_{5}}{Q_{5}-2}
$$

\subsection{Spectrum of strings on $A d S_{3}$}

From the fact that WZW action admits the $S L(2, R)$ current algebra we see that the physical spectrum of a string in $A d S_{3}$ must be in unitary representations of the current We construct the unitary representation of the $S L(2, R)$ current algebra by first constructing unitary representation of the global part of the $S L(2, R)$. This is given by

$$
\left[J_{0}^{3}, J_{0}^{ \pm}\right]= \pm J_{0}^{ \pm} \quad\left[J_{0}^{+}, J_{0}^{-}\right]=-2 J_{0}^{3}
$$

From this algebra we see states are classified by the eigen values of $J_{0}^{3}$ which we denote by $m$ and $j$ which is related to the Casimir $c_{2}=\frac{1}{2}\left(J_{0}^{+} J_{0}^{-}+J_{0}^{-} J_{0}^{+}\right)-\left(J_{0}^{3}\right)^{2}$ by $c_{2}=-j(j-1)$.

Unitary representations of $S L(2, R)$ fall in five classes ${ }^{23}$ :

1. Identity:

The trivial representation $|0\rangle$. This representation has $j=0, m=0$ and $J_{0}^{ \pm}|0\rangle=0$.

2. Principal discrete representations (lowest weight):

These are representation of the form

$$
\mathcal{D}_{j}^{+}=\{|j ; m\rangle: m=j, j+1, j+2 \cdots\},
$$

\footnotetext{
${ }^{23}$ See [179] for a review.
} 
Here $|j ; j\rangle$ is annihilated by $J_{0}^{-}$. The tower of states over $|j ; j\rangle$ is built by the repeated action of $J_{0}^{+}$. The norm of these states is positive and the representation is unitary if $j$ is real and $j>0 . j$ is restricted to be half integer if we are considering representation of the group $S L(2, R)$, however for the universal cover of $S L(2, R)$ which is our interest, $j$ can be any positive integer.

3. Principal discrete representations (highest weight):

These are representation of the form

$$
\mathcal{D}_{j}^{-}=\{|j ; m\rangle: m=-j,-j-1,-j-2, \cdots\},
$$

where $|j ; j\rangle$ is annihilated by $\left.J_{0}^{+}\right\rangle$. The representation has positive norm and is unitary if $j$ is real and $j>0$. This representation is the charge conjugate of $\mathcal{D}_{j}^{+}$.

4. Principal continuous representations:

A representation is of the form

$$
\mathcal{C}_{j}^{\alpha}=\{|j, \alpha ; m\rangle: m=\alpha, \alpha \pm 1, \alpha \pm 2, \cdots\}
$$

Without loss of generality, we can restrict $0 \leq \alpha<1$. The representation has positive norm and is unitary if $j=1 / 2+i s$ where $s$ is real.

5. Complementary representations:

These are of the form

$$
\mathcal{E}_{j}^{\alpha}=\{|j, \alpha ; m\rangle: m=\alpha, \alpha \pm 1, \alpha \pm 2, \cdots\}
$$

Again without loss of generality we can restrict $0 \leq \alpha<1$ The representation has positive norm and is unitary if $j$ is real and $j(1-j)>\alpha(1-\alpha)$.

Among these representations, we restrict to those which admit square integrable wave functions in the point particle limit. As $A d S_{3}$ is non-compact, squareintegrability refers to delta function normalizable wave functions. This imposes the restriction $j>1 / 2^{24}$. It is known that $\mathcal{C}_{j=1 / 2+i s}^{\alpha} \otimes \mathcal{C}_{j=1 / 2+i s}^{\alpha}$ and $\mathcal{D}_{j}^{ \pm} \otimes \mathcal{D}_{j}^{ \pm}$ with $j>1 / 2$ form the complete basis of square integrable wave functions on $A d S_{3}$. So it is sufficient to work with these representations only. Let us call a unitary representation of $S L(2, R)$ with this restriction as $\mathcal{H}$.

Now that we have the unitary representation of the global part of the current algebra we can obtain the unitary representation of the $S L(2, R)$ current algebra by considering $\mathcal{H}$ as its primary states which are annihilated by $J_{n}^{3}, J_{n}^{ \pm}$where $n>0$.

\footnotetext{
${ }^{24}$ This condition is also the condition for the Breitenlohner-Freedman bound 180 on $\mathcal{D}_{j}^{ \pm}$which states the mass of a scalar in $A d S_{3}$ is given by $m^{2}=j(j-1) \geq-\frac{1}{4}$.
} 
Then the full representation is obtained by the action of $J_{n}^{3}, J_{n}^{ \pm}$with $n<0$ on $\mathcal{H}$. We denote this full representation by $\hat{\mathcal{D}}_{j}^{ \pm}$and $\hat{\mathcal{C}}_{j=1 / 2+i s}^{\alpha}$. In general, representation of the $S L(2, R)$ current algebra contains negative norm states. String theory on $A d S_{3}$ is consistent if one can remove these negative states by imposing the Virasoro constraint on the Hilbert space for a single string state. If we consider the bosonic string theory on $A d S_{3}$ then the Virasoro constraint is given by

$$
\left.\left(L_{n}^{\text {total }}-\delta_{n, 0}\right) \mid \text { Physical }\right\rangle=0, \quad n \geq 0,
$$

Here $L_{n}^{\text {total }}$ refers to the Virasoro generator of the $c=26$ conformal field theory including the $S L(2, R)$ WZW model. It has been shown that there are no negative norm states for $\hat{\mathcal{D}}_{j}^{ \pm}$with $0<j<k / 2$ and $\hat{\mathcal{C}}_{j=1 / 2+i s}^{\alpha}$ (See [181, 182, 174] for a list of references).

In [174] it was seen that the $S L(2, R)$ WZW model admits a symmetry given by

$$
J_{n}^{3}=\tilde{J}_{n}^{3}+\frac{k}{2} w \delta_{n, 0}, \quad J_{n}^{+}=\tilde{J}_{n-w}^{+}, \quad J_{n}^{-}=\tilde{J}_{n+w}^{-},
$$

where $w$ is any integer. The map of $J$ 's to $\tilde{J}$ 's preserves the commutation relations (10.17). The Virasoro generators $\tilde{L}_{n}$ can be found using the Sugawara construction and they are related to $L_{n}$ 's by the map

$$
L_{n}=\tilde{L}_{n}-w \tilde{J}_{n}^{3}-\frac{k}{4} w^{2} \delta_{n}, 0
$$

This symmetry of the $S L(2, R)$ WZW model is called spectral flow. It is the same symmetry as the one which allowed the generation of new classical solutions from old ones in section 10.2. This can be seen easily by computing the change in the stress energy tensor and the $S L(2, R)$ generators under the map (10.14). They are identical to (10.27) and (10.26) respectively. The spectral flow maps one representation to another. For the case of a compact group like $S U(2)$ it does not generate a new representation, but for the non-compact group $S L(2, R)$ it generates new representations. Let us call the resulting representations $\hat{\mathcal{D}}_{\tilde{j}}^{ \pm, w}$ and $\hat{\mathcal{C}}_{1 / 2+i s}^{\alpha, w}$, where $\tilde{j}$ denotes the $S L(2, R)$ spin before the flow. The representations obtained by the spectral flow also have negative norms states. It has been shown in [174], that there are no negative norm states for representation obtained from spectral flow for $1 / 2<\tilde{j}<(k-1) / 2$.

Now we have the ingredients to state the proposal for the spectrum of strings on $A d S_{3}$ [174]. The spectrum consists of two kinds of representations, the spectral flow of the continuous representation with the same amount of spectral flow on the left and right $\hat{\mathcal{C}}_{1 / 2+i s, L}^{\alpha, w} \otimes \hat{\mathcal{C}}_{1 / 2+i s, R}^{\alpha, w}$ along with the spectral flow of the discrete representations $\hat{\mathcal{D}}_{\tilde{j}, L}^{ \pm, w} \otimes \hat{\mathcal{D}}_{\tilde{j}, R}^{ \pm, w}$. The value of $\tilde{j}$ is restricted to be $1 / 2<\tilde{j}<(k-1) / 2$. These representations should be tensored with the representations of the internal CFT which contributes to the net central charge. We then have to impose the 
Virasoro constraints. The expressions for the energy and the virasoro constraints for both the discrete and the continuous representation are given in 174. This proposal was verified in 175 by reading out the spectrum from the modular invariant one-loop partition function in thermal $A d S_{3}$ background. The discrete and continuous states obtained form the one-loop partition function was in agreement with the above proposal. We will review [175] in section 10.5.

\subsection{Strings on Euclidean $A d S_{3}$}

In this section we formulate string theory on Euclidean $A d S_{3}$, we denote Euclidean $A d S_{3}$ by $\mathbf{H}$ (see Appendix $\mathrm{C}$ ). Again we will restrict our attention to a single Poincare patch in $\mathrm{H}$. Consider the following coordinate redefinition of the coordinates in the Euclidean version of (10.4)

$$
U=e^{\phi} \quad \gamma=i t+x_{5} \quad \bar{\gamma}=-i t+x_{5}
$$

Then the metric on $\mathrm{H}$ becomes (cf. (C.35) with $h=e^{-\phi}$ )

$$
d s^{2}=l^{2}\left(d \phi^{2}+e^{2 \phi} d \gamma d \bar{\gamma}\right)
$$

Here $l^{2}=\alpha^{\prime} Q_{5}$. The value of the B-field can be read out again from (10.4) and is given by

$$
B=l^{2} e^{2 \phi} d \gamma \wedge d \bar{\gamma}
$$

The B-field is necessary for worldsheet conformal invariance. The B-field in Euclidean $A d S_{3}$ is imaginary. We work with a Euclidean worldsheet theory. This makes the contribution of B-field to the world sheet Lagrangian real. The world sheet action is given by

$$
S=\frac{l^{2}}{2 \pi \alpha^{\prime}} \int d^{2} z\left(\partial \phi \bar{\partial} \phi+e^{2 \phi} \bar{\partial} \gamma \partial \gamma\right)
$$

Let us write this action in a more convenient form. Introducing auxiliary fields $\beta$ and $\bar{\beta}$ of weights $(1,0)$ and $(0,1)$ we can write the action as

$$
S=\frac{l^{2}}{2 \pi \alpha^{\prime}} \int d^{2} z\left(\partial \phi \bar{\partial} \phi+\beta \bar{\partial} \gamma+\bar{\beta} \partial \bar{\gamma}-e^{-2 \phi} \beta \bar{\beta}\right)
$$

Integrating out the auxiliary fields $\beta$ and $\bar{\beta}$ in the above equation we obtain (10.31). Scaling $\phi$ so that it has the canonical normalization and taking into account of the measure we obtain.

$$
S=\frac{1}{4 \pi} \int d^{2} z\left(\partial \phi \bar{\partial} \phi-\frac{2}{\alpha_{+}} \hat{R} \phi+\beta \bar{\partial} \gamma+\bar{\beta} \partial \bar{\gamma}-\beta \bar{\beta} \exp \left(-\frac{2}{\alpha_{+}} \phi\right)\right)
$$

Here $\alpha_{+}=\sqrt{2 Q_{5}-4}$ and $\hat{R}$ is the world sheet curvature. Notice that the coefficient of the exponent has been renormalized. The action becomes free at $\phi \rightarrow \infty$ which 
is near the boundary of $A d S_{3}$. The world sheet propagators of the fields in (10.33) are

$$
\langle\phi(z) \phi(0)\rangle=-\log |z|^{2} \quad\langle\beta(z) \gamma(0)\rangle=\frac{1}{z}
$$

This action (10.33) admits a $S L(2, R) \times S L(2, R)$ current algebra. This representation of the $S L(2, R)$ current algebra is called the Wakimoto representation [183. The holomorphic currents are given by

$$
\begin{aligned}
J^{3} & =\beta \gamma+\frac{\alpha_{+}}{2} \partial \phi \\
J^{+} & =\beta \gamma^{2}+\alpha_{+} \gamma \partial \phi+Q_{5} \partial \gamma \\
J^{-} & =\beta
\end{aligned}
$$

Similar definitions for the antiholomorphic currents exist. The modes of these currents generate the $S L(2, R)$ current algebra given in (10.17) with the same central charge as given in (10.19).

\subsubsection{The long string worldsheet algebra}

We now derive the worldsheet degrees of freedom of the long string solution in $\mathrm{H}$. Using supersymmetry one can derive the worldsheet theory of the long string exactly, unlike our classical analysis in section 7.1. We will follow the discussion given in [114]. The long string solution in the static gauge is given by [114, 139, 174]

$$
\phi=\phi_{0}, \quad \gamma(z, \bar{z})=z, \quad \bar{\gamma}(\bar{z}, \bar{z})=\bar{z}
$$

The ghosts corresponding to $\gamma$ and $\bar{\gamma}$ decouple 114. Thus the bosonic worldsheet degrees of freedom of the long string will be the coordinate $\phi$ characterizing its radial position in $\mathrm{H}$, the coordinates on the sphere $S^{3}$ which forms an $S U(2)$ current algebra $j^{a}, a=1, \ldots, 3$ and the coordinates of $T^{4}$. The 4 fermionic partners of the coordinates on $T^{4}$ with the bosons form a $\mathcal{N}=(4,4)$ superconformal algebra with central charge 6 . The superpartners of the coordinate $\phi$ and the $S U(2)$ current algebra $j^{a}$ of the sphere $S^{3} 4$ free fermions $S^{\mu}$ with $\mu=1, \ldots, 4$. From the fact that the radius of $S^{3}$ is $Q_{5}$ the we know that the $S U(2)$ current algebra is at level $Q_{5}-2^{25}$. These arguments lead us to the following operator product expansions among the fields

$$
\begin{aligned}
S^{\mu}(z) S^{\nu}(w) & =-\frac{\delta^{\mu \nu}}{z-w} \\
\partial \phi(z) \partial \phi(w) & =-\frac{1}{(z-w)^{2}} \\
j^{a}(z) j^{b}(w) & =-\frac{\delta^{a b}\left(Q_{5}-2\right)}{2(z-w)^{2}}+\frac{\epsilon^{a b c} j^{c}}{(z-w)}
\end{aligned}
$$

\footnotetext{
${ }^{25}$ The shift in the level is because we have used decoupled fermions (see for instance in [184]).
} 
To keep our discussion less cumbersome we have ignored the anti-holomorphic fields. As the space time preserves 16 supersymmetries we should construct out of these field an $\mathcal{N}=(4,4)$ superconformal algebra. It is known [184, 114] that the following construction has the required properties of the long sting.

$$
\begin{aligned}
T & =-\frac{1}{2} \partial S^{\mu} S^{\mu}-\frac{j^{a} j^{a}}{Q_{5}}-\frac{1}{2} \partial \phi \partial \phi+\frac{\sqrt{2}\left(Q_{5}-1\right)}{2 \sqrt{Q_{5}}} \partial^{2} \phi \\
J^{a} & =j^{a}+\frac{1}{2} \eta_{\mu \nu}^{a} S^{\mu \nu} \\
G^{\mu} & =\frac{\sqrt{2}}{2} \partial \phi S^{\mu}-\frac{2}{\sqrt{Q_{5}}} \eta_{\mu \nu}^{a} j^{a} S^{\nu}+\frac{1}{6 \sqrt{Q_{5}}} \epsilon_{\mu \nu \rho \sigma} S^{\nu} S^{\rho} S^{\sigma}-\frac{Q_{5}-1}{\sqrt{Q_{5}}} \partial S^{\mu}
\end{aligned}
$$

These generators form an $\mathcal{N}=(4,4)$ superconformal algebra given in (5.2) with the following definition for the two component super charge in (5.2)

$$
G^{a}=\left(G^{1}+i G^{2}, G^{3}-i G^{4}\right)
$$

The following points are worth noting

1. Note from the definition of the stress energy tensor that the field $\phi$ is a linear dilaton with background charge $Q=\sqrt{\frac{2}{Q_{5}}}\left(Q_{5}-1\right)$. This is precisely the background charge of the linear dilaton theory for the case of a single D1 brane splitting off the D1-D5 bound state (see below (7.40)).

2. The central charge of this algebra is $6\left(Q_{5}-1\right)$ and not 6 , as one would have expected if this algebra was describing the spacetime geometry

3. Note also that the R-symmetry generators in (10.38) involve the bosonic fields $j^{a}$ which corresponds to the symmetry of $S^{3}$. This is a characteristic of the Higgs branch [113].

\subsection{Strings on the thermal $A d S_{3}$}

To discuss thermal boundary conditions on $A d S_{3}$ it is convenient to parameterize Euclidean $A d S_{3}$ using the following coordinates

$$
\gamma=v e^{\phi} \quad \bar{\gamma}=\bar{v} e^{\phi}
$$

In terms of these new coordinate the metric on $\mathrm{H}$ becomes (see $(\underline{10.29}),(\overline{C .35})$ )

$$
d s^{2}=l^{2}\left(d \phi^{2}+(d v+v d \phi)(d \bar{v}+\bar{v} d \phi)\right)
$$

The worldsheet action with the B-field given by $(10.30)$ is

$$
S=\frac{Q_{5}}{2 \pi} \int d^{2} z(\partial \phi \bar{\partial} \phi+(\partial \bar{v}+\partial \phi \bar{v})(\bar{\partial} v+\bar{\partial} \phi v))
$$


Thermal $A d S_{3}$ is then defined by the following identifications (see Appendix C.4)

$$
\begin{gathered}
v \sim v e^{i \mu \beta} \\
\bar{v} \sim \bar{v} e^{-i \mu \beta} \\
\phi \sim \phi+\beta
\end{gathered}
$$

Here $\beta$ is the inverse temperature and $\mu$ is the chemical potential.

We now set up the one-loop evaluation of the partition function on $\mathrm{H}$ [175]. From this it is easy to evaluate the space time free energy and thus determine the spectrum of strings in $A d S_{3}$. We have to evaluate the path integral on a torus with modular parameter $\tau$. The conformal field theory consists of the worldsheet Lagrangian (10.42) with the identifications (10.43), the $b c$ ghosts and an internal conformal field theory. Let the partition function of the internal conformal field theory be given by

$$
\mathcal{Z}_{M}=(q \bar{q})^{-\frac{c_{\text {int }}}{24}} \sum_{h, \bar{h}} D(h, \bar{h}) q^{h} \bar{q}^{\bar{h}}
$$

where $q=e^{2 \pi i \tau}, D(h, \bar{h})$ is the degeneracy of the state with weight $(h, \bar{h})$. Putting this partition function and that of the $b, c$ ghosts together we get [175],

$$
\begin{aligned}
Z(\beta, \mu) & =\frac{\beta(k-2)^{\frac{1}{2}}}{8 \pi} \int_{0}^{\infty} \frac{d \tau_{2}}{\tau_{2}^{3 / 2}} \int_{-1 / 2}^{1 / 2} d \tau_{1} e^{4 \pi \tau_{2}\left(1-\frac{1}{4(k-2)}\right)} \sum_{h, \bar{h}} D(h, \bar{h}) q^{h} \bar{q}^{\bar{h}} \\
& \times \sum_{m=1}^{\infty} \frac{e^{-(k-2) m^{2} \beta^{2} / 4 \pi \tau_{2}}}{|\sinh (m \hat{\beta} / 2)|^{2}}\left|\prod_{n=1}^{\infty} \frac{1-e^{2 \pi i n \tau}}{\left(1-e^{m \hat{\beta}+2 \pi i n \tau}\right)\left(1-e^{-m \hat{\beta}+2 \pi i n \tau}\right)}\right|^{2}
\end{aligned}
$$

where $\hat{\beta}=\beta+i \mu \beta$.

From the one-loop partition function it is easy to extract the spacetime free energy $F$, which is given by $Z(\beta, \mu)=-\beta F$. One can rewrite the free energy as a sum over states in the single particle string Hilbert space $\mathcal{H}$

$$
F(\beta, \mu)=\frac{1}{\beta} \sum_{\text {string } \in \mathcal{H}} \log \left(1-e^{-\beta\left(E_{\text {string }}+i \mu l_{\text {string }}\right)}\right)
$$

where $E_{\text {string }}$ and $l_{\text {string }}$ are the energy and angular momentum of the string state. One can compare (10.45) and (10.46) and show that the spectrum is precisely the one proposed by [174] which was discussed in section 10.3. 


\section{Applications of $\mathrm{AdS}_{3}-\mathrm{CFT}_{2}$ duality}

The microscopic derivation (Section 8) of Hawking radiation from the D1-D5 black hole shows that the microstates of the black hole are to be identified with states of the "boundary CFT". One way to understand this is to note that [42, 41] in the black hole description (large 'tHooft coupling $g_{s} Q$ ) the propagation of closed string quanta in the curved geometry (see Section 3) consists of (a) free propagation in the asymptotically flat region and (b) propagation in $\mathrm{AdS}_{3}$ geometry (throat region); in the weak coupling description the closed string quanta $\left(\mathrm{a}^{\prime}\right)$ propagate freely in flat space and $\left(b^{\prime}\right)$ occasionally interact with the D1-D5 system which, for the low energy scales associated with Hawking quanta, is described by a CFT. Since we are describing the same physical process as $(\mathrm{a})+(\mathrm{b})$ at strong coupling and as $\left(a^{\prime}\right)+\left(b^{\prime}\right)$ at weak coupling and since (a) is clearly equivalent to $\left(a^{\prime}\right)$, we say that (b) is equivalent (dual) to $\left(\mathrm{b}^{\prime}\right)$. That is, as seen by a closed string probe, supergravity in $\mathrm{AdS}_{3}$ is equivalent to the CFT of the D1-D5 system. This was indeed the reasoning behind the discovery of the AdS/CFT duality 442.

While the above equivalence gives us important insight in the context of the full geometry including the asymptotically flat part, the equivalence between asymptotically $\mathrm{AdS}_{3}$ spaces and the boundary $\mathrm{CFT}_{2}$ has a number of important applications. This subject has been discussed in fair amount of detail in [41]. Our discussion here should be regarded as complementary to it; we will focus here mainly on (a) thermodynamics and phase transitions in $\mathrm{AdS}_{3}$ and (b) black hole formation by particle collision. We will begin with (a).

\subsection{Hawking-Page transition in $\mathrm{AdS}_{3}$}

In the context of flat space, it is well-known [32, 185] that a thermal state in flat Minkowski space, no matter how low the temperature, is unstable to formation of a black hole:

- For a flat space with infinite volume, the mass of a thermal state is infinite; therefore the state will gravitationally collapse to a black hole. The conclusion remains true even if one restricts to a large but finite volume.

- Furthermore, because of the negative specific heat implied by $T=1 /(8 \pi G M)$ (Eq.(1.2)), a black hole can only be in an unstable equilibrium with radiation at the same temperature (in a sufficiently large volume to keep the temperature constant). Any fluctuation resulting in an increase of the black hole mass would reduce its temperature below that of its surroundings, causing more absorption than emission so that the black hole continues to grow. This implies a breakdown of the canonical ensemble. 
As found in [186], the situation is different in an AdS space. Let us write the metric of $\mathrm{AdS}_{3}$ as (see (2.71), (C.7))

$$
d s^{2}=-d t^{2}\left(1+\frac{r^{2}}{l^{2}}\right)+d r^{2}\left(1+\frac{r^{2}}{l^{2}}\right)^{-1}+r^{2} d \phi^{2}
$$

and that of BTZ as (see (2.77), C.11), (C.13))

$$
d s^{2}=-\left[\frac{r^{2}}{l^{2}}-M+\left(\frac{J}{2 r}\right)^{2}\right] d t^{2}+\left[\frac{r^{2}}{l^{2}}-M+\left(\frac{J}{2 r}\right)^{2}\right]^{-1} d r^{2}+r^{2}\left(\frac{-J}{2 r^{2}} d t+d \phi\right)^{2}
$$

where $M$ and $J$ refer to the mass and angular momentum of the BTZ black hole $(J \leq M)$. The arguments of [186], applied to the present case, state that a thermal state describing radiation in $\mathrm{AdS}_{3}$ at a finite temperature has a finite mass. For $l \gg 1$ the energy density of radiation is given approximately by the formula $\rho \propto T^{3}$ where $T$ is the locally measured temperature $T=T_{0}\left(1+\frac{r^{2}}{l^{2}}\right)^{-1 / 2}$ which, for $r \gg l$, goes as $T \propto 1 / r$. The total energy is given by the integral $M \approx \int l d r d \phi \rho$ which is clearly finite since $\rho \propto r^{-3}$ at large distances $r$. Thus, unlike in flat space, thermal radiation in $\mathrm{AdS}_{3}$ can be a stable configuration, if its (free) energy is less than that of a black hole at the same temperature (see below).

We will also see below that the BTZ black hole has a positive specific heat (see, e.g. the formula for the temperature (C.45)), unlike the Schwarzschild black hole. Thus a stable equilibrium between a BTZ black hole and radiation in $\mathrm{AdS}_{3}$ is possible; a fluctuation resulting in an increase of mass of the black hole increases its temperature above that of the radiation, causing more emission than absorption by the black hole, thus restoring its energy back to the equilibrium value.

\section{Euclidean Free energy from supergravity}

As we argued in Section 2, the $\mathrm{AdS}_{3}$ and BTZ spacetimes (11.1),(11.2) (times $S^{3} \times K$ ) are near-horizon limits of solutions of type IIB supergravity (namely the D1-D5 string and the D1-D5 black hole). In fact they are exact solutions of type IIB supergravity in their own right. Stated differently, $\mathrm{AdS}_{3}$ and BTZ are solutions of three-dimensional supergravity obtained by a Kaluza-Klein reduction of type IIB theory on $S^{3} \times K$.

Let us elaborate this a bit more. The action for pure anti-de Sitter supergravity, based on the super group $S U(1,1 \mid 2) \times S U(1,1 \mid 2)$ (see Section 6), in a three-dimensional spacetime with cosmological constant $\Lambda=-1 / l^{2}<0$, is given by (see 136, 187]) ${ }^{26}$

$$
S=\frac{1}{16 \pi G_{N}^{(3)}} \int d^{3} x\left[e R+\frac{2}{l^{2}} e-\epsilon^{\mu \nu \rho} \bar{\psi}_{\mu} \mathcal{D}_{\nu} \psi_{\rho}-8 l \epsilon^{\mu \nu \rho}\left(A_{\mu}^{i} \partial_{\nu} A_{\rho}^{i}-\right.\right.
$$

\footnotetext{
${ }^{26}$ Recently the three-dimensional $S O(4)$ gauged supergravity which provides the coupling of the pure anti-de Sitter supergravity based on the supergroup $S U(1,1 \mid 2) \times S U(1,1 \mid 2)$ to the lowest matter multiplets including the massless scalar fields has been constructed in 188.
} 


$$
\left.\left.\frac{4 i \epsilon_{i j k}}{3} A_{\mu}^{i} A_{\nu}^{j} A_{\rho}^{k}\right)-\epsilon^{\mu \nu \rho} \bar{\psi}_{\mu}^{\prime} \mathcal{D}^{\prime}{ }_{\nu} \psi_{\rho}^{\prime}+8 l \epsilon^{\mu \nu \rho}\left(A_{\mu}^{\prime i} \partial_{\nu} A_{\rho}^{\prime i}-\frac{4 i \epsilon_{i j k}}{3} A_{\mu}^{\prime i} A_{\nu}^{\prime j} A_{\rho}^{\prime k}\right)\right](1
$$

Here $G_{N}^{(3)}$ is the three-dimensional Newton's constant, and $\mathcal{D}_{\nu}=\partial_{\nu}+\omega_{a b \nu} \gamma^{a b} / 4-$ $e_{a \nu} \gamma^{a} /(2 l)-2 A_{\nu}^{i} \sigma^{i}$ and $\mathcal{D}_{\nu}^{\prime}=\partial_{\nu}+\omega_{a b \nu} \gamma^{a b} / 4+e_{a \nu} \gamma^{a} /(2 l)-2 A_{\nu}^{\prime i} \sigma^{i}$. The basic fields appearing in the Lagrangian are the vielbein $e_{\mu}^{a}, \psi_{\rho}, A_{\mu}^{i}, \psi_{\mu}^{\prime}$ and $A_{\mu}^{\prime i}$. The $\omega^{\prime}$ 's are spin connections.

As we remarked before (11.3), the same three-dimensional supergravity can be obtained from type IIB string theory compactified on $K \times S^{3}$ (with constant flux on $S^{3}$ ). This identifies the cosmological constant and Newton's constant in terms of type IIB parameters and the parameters of compactification (cf. Eqn. (2.73)):

$$
\begin{aligned}
G_{N}^{(3)} & =\frac{4 \pi^{4} g_{s}^{2}}{V_{4} l^{3}} \\
l^{4} & =\frac{16 \pi^{4} g_{s}^{2} Q_{1} Q_{5}}{V_{4}}
\end{aligned}
$$

where $V_{4}$ is the volume of $T^{4}$ and $g_{s}$ is the string coupling (we are working in the units $\left.\alpha^{\prime}=1\right)$.

From our remarks above, it is obvious that $\mathrm{AdS}_{3}$ and BTZ are solutions of (11.3). We will now use this supergravity Lagrangian to calculate the Euclidean free energy. Our method will be similar in spirit to that of [28, 186, 189]. The presentation will more closely follow [190, 90, 191].

Let us recall that the free energy of $\mathrm{AdS}_{3}$ supergravity is given by

$$
Z_{\text {sugra }}=\int \mathcal{D}_{\alpha}[\text { fields }] \exp \left[-S_{E}\right]
$$

where $S_{E}$ is the Euclidean version of the action written in (11.3).

\section{Boundary Conditions:}

In the above equation, $\alpha$ denotes boundary conditions that define the functional integral. The semiclassical evaluation of the functional integral is performed by summing over the saddle point configurations which satisfy the given boundary conditions.

The boundary conditions are specified as follows:

1. Asymptotically $\mathrm{AdS}_{3}$ Euclidean metrics satisfy the boundary condition:

$$
d s^{2} \stackrel{r \rightarrow \infty}{\rightarrow} d r^{2} / r^{2}+r^{2}|d \phi+i d \mathrm{t}|^{2}
$$

where $\phi, \mathrm{t}$ satisfy the periodicity conditions:

$$
\phi+i \mathrm{t} \equiv \phi+i \mathrm{t}+2 \pi(n+m \tau)
$$


This defines the (conformal) boundary of the space to be a torus with modular parameter $\tau$.

For Euclidean $\mathrm{AdS}_{3}$, Eqns. (C.24), C.32) imply that the modular parameter for the boundary torus is

$$
\tau=i \beta /(2 \pi), \quad \beta=1 / T-i \Phi
$$

where the complex temperature $\beta$ (see (C.33) relates to a partition function of the form $\operatorname{Tr} \exp [-H / T+i \Phi J]$.

For Euclidean BTZ, on the other hand, Eqns. (C.37), (C.44) imply that the modular parameter for the boundary torus (cf. (11.6)) is now

$$
\tilde{\tau}=i \beta / 2 \pi=-1 / \tau
$$

where $\tau$ is given by (C.41).

The inverse relation (11.9) between the modular parameters $\tau, \tilde{\tau}$ arises because of the difference in our identifications of space and Euclidean time in (C.31), (C.40). Indeed from this viewpoint, Euclidean BTZ is a special case of an $\mathrm{SL}(2, \mathrm{Z})$ family of instanton configurations [90, 191]. In the more general case we replace the parameter $\tau$ in (C.38) by

$$
\tilde{\tau}=\frac{a \tau+b}{c \tau+d}, \quad\left(\begin{array}{ll}
a & b \\
c & d
\end{array}\right) \in S L(2, Z)
$$

and $(\overline{C .40})$ is replaced by

$$
2 u=\frac{i}{c \tau+d}(\phi+i \mathrm{t})
$$

In 191 an elliptic genus calculated from the boundary CFT is interpreted as a sum over this entire $\mathrm{SL}(2, \mathrm{Z})$ family of gravitational instantons. In our simplified treatment below, only $\mathrm{AdS}_{3}$ and $\mathrm{BTZ}$ configurations will dominate the path integral. As an upshot of the above discussion is the relation (cf. (11.8), (C.44)

$$
Z_{A d S}(\tau=i \beta / 2 \pi)=Z_{B T Z}\left(\tau=i \beta_{0} / 2 \pi\right) \quad \beta_{0}=\left(4 \pi^{2} / \beta\right)
$$

We should note that for Euclidean BTZ black hole, the complex temperature gets fixed by the geometry (see (C.41)), whereas for Euclidean $\mathrm{AdS}_{3}$ the complex temperature is for us to specify.

2. Gauge field: the boundary condition on the gauge fields will not play an important role in the following discussion (see Eq. (5.7) of 191 for details). 
3. Fermions: the above discussion, especially (11.6), (11.7) points to two nontrivial cycles of the boundary torus. The fermion fields can have either periodic (P) or antiperiodic (AP) boundary conditions along either cycle. Thus we can have any of the four boundary conditions (P, P), (P, A), (A, P), (A, $\mathrm{A})$, where by convention the entry denotes boundary condition along the cycle $\phi+i \mathrm{t} \rightarrow \phi+i \mathrm{t}+2 n \pi$ and the second entry denotes boundary condition along $\phi+i \mathrm{t} \rightarrow \phi+i \mathrm{t}+i \beta, \tau=i \beta / 2 \pi$. In the language of 2D SCFT, a periodic boundary condition correspond to Ramond (R) fermions and an antiperiodic b.c. corresponds to Neveu-Schwarz (NS) fermions. By the remarks at the end of Section 2.6.2 (see also Section 6) we find that the BTZ fermions correspond to Ramond boundary conditions along the "space" cycle; thus a standard Euclidean partition function in BTZ should correspond to the boundary condition $(\mathrm{P}, \mathrm{AP})$ (thermal fermions represented by $\operatorname{Tr}\left(e^{-\beta H}\right)$ happen to be antiperiodic along the "time" cycle). We will compute below such a partition function. By the duality (11.12) this should be related to (AP,P) for the fermions in $\mathrm{AdS}_{3}$. This is consistent with the remarks at the end of Section 2.6.2 (see also Section 6) that $\mathrm{AdS}_{3}$ fermions are represented by NS boundary conditions at the boundary.

\section{Finding the Saddle points}

We will evaluate (11.5) by finding saddle points of the action subject to the specific boundary conditions mentioned above. By virtue of the equation of motion $R=-6 / l^{2}$, the Euclidean action $S$ of a classical spacetime $X$ is simply its volume times a constant. To be precise,

$$
\begin{aligned}
S(X) & =\frac{1}{4 \pi l^{2} G_{N}^{(3)}} \operatorname{Vol}(X) \\
\operatorname{Vol}(X) & =\int_{0}^{\beta} d \mathrm{t} \int_{r_{0}}^{R} d r \int_{0}^{2 \pi} d \phi \sqrt{g}
\end{aligned}
$$

The ranges of $\phi, \tau$ follow from the identifications mentioned above. The lower limit $r_{0}$ of the $r$-integral is identically zero for $\mathrm{AdS}_{3}$ and the conical spaces, whereas for BTZ it denotes the location of the horizon (the Euclidean section is defined only upto the horizon). The upper limit $R$ is kept as an infrared regulator to make the volume finite. We will in practice only be interested in free energies relative to $\mathrm{AdS}_{3}$ and the $R$-dependent divergent term will disappear from that calculation.

Free energy of BTZ:

We will now compute the free energy of BTZ relative to $\mathrm{AdS}_{3}$ (in a manner similar to 92, 189]). As detailed in Appendix $\mathrm{C}$, the $\mathrm{AdS}_{3}$ solution can be at any temperature $\beta$ (C.33) while the temperature of the black hole is fixed to be 
$\beta_{0}$ (C.44),(C.41) which is given by the geometry. To compare with the $A d S_{3}$ background one must adjust $\beta$ so that the geometries of the two manifolds match at the hypersurface of radius $R$ (in other words, we must use the same infrared regulator on all saddle points of the functional integral). This gives the following relation

$$
\begin{aligned}
\beta_{0} & =\beta \sqrt{\frac{1+l^{2} / R^{2}}{1-M^{2} l^{2} / R^{2}}} \\
S(B T Z)-S\left(A d S_{3}\right) & =\frac{1}{4 \pi l^{2} G_{N}^{(3)}}\left[\int_{B T Z} d^{3} x \sqrt{g}-\int_{A d S_{3}} d^{3} x \sqrt{g}\right] \\
& =\frac{1}{4 \pi l^{2} G_{N}^{(3)}}\left[\pi \beta_{0}\left(R^{2}-r_{+}^{2}\right)-\pi \beta R^{2}\right]
\end{aligned}
$$

Substituting the value of $\beta_{0}$ in terms of $\beta^{27}$ and taking the limit $R \rightarrow \infty$ we obtain

$$
S(B T Z)-S\left(A d S_{3}\right)=\frac{1}{4 \pi l^{2} G_{N}^{(3)}}\left[\pi \beta_{0} l^{2}-\pi^{2} r_{+} l^{2}\right]
$$

where the complex temperature $\beta_{0}$ is given by Eqns. (C.41), (C.45). Using these variables the difference in the action becomes (identifying $\beta_{0}$ with $\beta$ ) [190

$$
S(B T Z)-S\left(A d S_{3}\right)=\frac{1}{4 \pi l^{2} G_{N}^{(3)}}\left[\frac{\pi}{2}\left(\beta+\beta^{*}\right) l^{2}-\pi^{3} l^{4}\left(\frac{1}{\beta}+\frac{1}{\beta^{*}}\right)\right]
$$

By using (11.12) we find

$$
\begin{aligned}
& S\left(\mathrm{AdS}_{3}\right)=\frac{1}{4 \pi l^{2} G_{N}^{(3)}}\left[\frac{\pi}{2}\left(\beta+\beta^{*}\right) l^{2}\right] \\
& S(B T Z)=\frac{1}{4 \pi l^{2} G_{N}^{(3)}} \pi^{3} l^{4}\left[\frac{1}{\beta}+\frac{1}{\beta^{*}}\right]
\end{aligned}
$$

It is clear that at low temperatures the $\mathrm{AdS}_{3}$ saddle point dominates the path integral, where at high temperatures the BTZ dominates. The transition from $\mathrm{AdS}_{3}$ at low temperature to BTZ at high temperature is the 3-dimensional analogue of the Hawking-Page transition.

\section{Euclidean Free energy from CFT}

\footnotetext{
${ }^{27}$ We should make a remark here to avoid any potential confusion between equations like (11.12) and (11.14); the former equation is a statement that the Euclidean partition function of $\mathrm{AdS}_{3}$, corresponds to a certain complex temperature, matches with that of BTZ at a different temperature. In calculating the supergravity partition function, however, we want to regard both as having the same temperature $(R \rightarrow \infty$ limit of (11.14) $)$; the difference between which bulk geometry corresponds to $\mathrm{AdS}_{3}$ and which to BTZ arises here by the choice of "space" and "time" (see Appendix C.4).
} 
The aim of this section is to calculate the partition function of the $(4,4)$ CFT on the orbifold $T^{4 Q_{1} Q_{5}} / S\left(Q_{1} Q_{5}\right)$. The partition function depends on the boundary conditions of the fermions of the CFT. We will first calculate the partition function when the bulk geometry is that of the BTZ black hole.

\section{CFT partition function corresponding to BTZ}

The fermions of the CFT are periodic along the angular coordinate of the cylinder if the bulk geometry is that of the BTZ black hole. This can be seen by observing that the zero mass BTZ black hole admits killing vectors which are periodic along the angular coordinate [93]. Therefore the zero mass BTZ black hole correspond to the Ramond sector of the CFT. The general case of the BTZ black hole with mass and angular momentum correspond to excited states of the CFT over the Ramond vacuum with

$$
\begin{aligned}
& L_{0}+\bar{L}_{0}=M l \\
& L_{0}-\bar{L}_{0}=J_{E}
\end{aligned}
$$

where $M$ and $J_{E}$ are the mass and the (Euclidean) angular momentum of the BTZ black hole. Therefore the partition function of the BTZ black hole should correspond to

$$
Z=\operatorname{Tr}_{R}\left(e^{2 \pi i \tau L_{0}} e^{2 \pi i \bar{\tau} \bar{L}_{0}}\right)
$$

The Hilbert space of the CFT on the orbifold $T^{4 Q_{1} Q_{5}} / S\left(Q_{1} Q_{5}\right)$ can be decomposed into twisted sectors labeled by the conjugacy classes of the permutation group $S\left(Q_{1} Q_{5}\right)$. The conjugacy classes of the permutation group consists of cyclic groups of various lengths. The various conjugacy classes and the multiplicity in which they occur in $S\left(Q_{1} Q_{5}\right)$ can be found from the solutions of the equation

$$
\sum_{n=0}^{Q_{1} Q_{5}} n N_{n}=Q_{1} Q_{5}
$$

where $n$ is the length of the cycle and $N_{n}$ is the multiplicity of the cycle. The Hilbert space is given by

$$
\mathcal{H}=\bigoplus_{\sum n N_{n}=Q_{1} Q_{5}} \bigotimes_{n>0} S^{N_{n}} \mathcal{H}_{(n)}^{P_{n}}
$$

$S^{N} \mathcal{H}$ denotes the symmetrized product of the Hilbert space $\mathcal{H}, N$ times. By the symbol $\mathcal{H}_{(n)}^{P_{n}}$ we mean the Hilbert space of the twisted sector with a cycle of length $n$ in which only states which are invariant under the projection operator

$$
P_{n}=\frac{1}{n} \sum_{k=1}^{n} e^{2 \pi i k\left(L_{0}-\bar{L}_{0}\right)}
$$

are retained. The values of $L_{0}$ or $\bar{L}_{0}$ in the twisted sector of length $n$ is of the form $p / n$ where $p$ is positive integer. This projection forces the value of $L_{0}-\bar{L}_{0}$ to be an 
integer on the twisted sector. It arises because the black hole can exchange only integer valued Kaluza-Klein momentum with the bulk [108].

The dominant contribution to the partition function arises from the maximally twisted sector. That is, from the longest single cycle of length $Q_{1} Q_{5}$. It is given by

$$
Z=\sum_{m, n} d\left(Q_{1} Q_{5} n+m\right) d(m) e^{2 \pi i n \tau} e^{2 \pi i m \tau / Q_{1} Q_{5}} e^{-2 \pi i m \bar{\tau} / Q_{1} Q_{5}}
$$

Where $d$ 's are the coefficients defined by the expansion

$$
Z_{T^{4}}=\left[\frac{\Theta_{2}(0 \mid \tau}{\eta^{3}(\tau)}\right]^{2}=\sum_{n \geq 0} d(n) e^{2 \pi i \tau n}
$$

In the above equation $Z_{T^{4}}$ is the partition function of the holomorphic sector of the CFT on $T^{4}$. We will first evaluate the sum

$$
P(m, \tau)=\sum_{n=0}^{\infty} d\left(Q_{1} Q_{5} n+m\right) e^{2 \pi i n \tau}
$$

For large values of $Q_{1} Q_{5}$ we can use the asymptotic form of $d\left(Q_{1} Q_{5} n+m\right)$

$$
d\left(Q_{1} Q_{5} n+m\right) \sim \exp \left(2 \pi \sqrt{Q_{1} Q_{5} n+m}\right)
$$

Substituting the above value of $d\left(Q_{1} Q_{5} n+m\right)$ in $P(m, \tau)$ we obtain a sum which has an integral representation as shown below.

$$
\begin{aligned}
P(m, \tau) & =\sum_{n=1}^{\infty} e^{2 \pi \sqrt{Q_{1} Q_{5} n+m}+2 \pi i n \tau}+d(m) \\
& =\mathcal{P} \frac{i}{2} \int_{-\infty}^{\infty} d w \operatorname{coth} \pi \omega e^{2 \pi \sqrt{i Q_{1} Q_{5} \omega+m}-2 \pi \omega \tau}+d(m)-\frac{e^{2 \pi \sqrt{m}}}{2}
\end{aligned}
$$

where $\mathcal{P}$ denotes "principal value" of the integral.

We are interested in the high temperature limit of the partition function. The leading contribution to the integral in the limit $\tau \rightarrow 0$ is

$$
P(m, \tau) \sim \sqrt{i \pi Q_{1} Q_{5} / \tau} e^{i \pi Q_{1} Q_{5} / 2 \tau-i 2 \pi m \tau / Q_{1} Q_{5}}
$$

Substituting the above value of $P(m, \tau)$ the partition function becomes

$$
Z=\sqrt{i \pi Q_{1} Q_{5} / \tau} \sum_{m=0}^{\infty} d(m) e^{-2 \pi i m \bar{\tau} / Q_{1} Q_{5}} \sim \exp \left(i \pi Q_{1} Q_{5}(1 / 2 \tau-1 / 2 \bar{\tau})\right)
$$

Thus the free energy at high temperatures is given by

$$
-\ln Z=\frac{-i \pi Q_{1} Q_{5}}{2}\left(\frac{1}{\tau}-\frac{1}{\bar{\tau}}\right)
$$


This exactly agrees with (11.19) with the identification $\tau=i \beta_{+} /(2 \pi l)$.

We will not attempt to calculate the $\mathrm{AdS}_{3}$ partition function here (with fermion b.c. (AP,P)), except to note that by using spectral flow arguments [191 it is possible to show that the CFT result agrees with (11.18). Indeed in 191] a more general agreement between the CFT elliptic genus and the corresponding $\mathrm{AdS}_{3}$ quantity is demonstrated for the entire SL(2,Z) family of solutions of which $\mathrm{AdS}_{3}$ and BTZ are special cases.

\subsection{Conical defects and particles in $A d S_{3}$}

In this section we will mention some of the continuing developments regarding point particle dynamics and black hole formation in $\mathrm{AdS}_{3}$.

It has long been noted [192, 193] that there is a one-parameter family of spacetimes, given by

$$
d s^{2}=l^{2}\left[-\left(\gamma+\frac{r^{2}}{l^{2}}\right) d t^{2}+\frac{d r^{2}}{\gamma+\frac{r^{2}}{l^{2}}}+r^{2} d \phi^{2}\right]
$$

which represent the so-called point masses in $\mathrm{AdS}_{3}$. These spacetimes are all asymptotically $\mathrm{AdS}_{3}$, and they interpolate between $\mathrm{AdS}_{3}$ and BTZ $(\gamma=-M=1$ is $\mathrm{AdS}_{3}, \gamma=-M \leq 0$ is $\mathrm{BTZ}(J=0)$, and $\gamma=-M \in(0,1)$ are the conical spaces). The conical spaces are called so because they have a defect angle $\Delta=2 \pi(1-\sqrt{\gamma})$ (see Appendix C). The parameter $\gamma$ is related to the "mass" of the point particle that causes the conical defect. To say it in more detail, consider a free particle (a geodesic) g in $\mathrm{AdS}_{3}$, given by

$$
\mathbf{Y}(s)=e^{s p^{a} \gamma_{a}}
$$

where we have used coordinates (C.3). The quantities $p^{a}$ play the role of "momenta" (see Section 11.2.1 for more detail). For a static particle

$$
p^{0}=m, p^{1}=p^{2}=0 .
$$

The geodesic (11.34) satisfies, in the coordinates (C.9),

$$
\phi(t)=0, \rho(t)=0 .
$$

We state without proof here (for more details see Section 11.2.1) that the gravitational back reaction of the point particle (11.34) amounts to cutting a wedge out of $\mathrm{AdS}_{3}$ and identifying the edges. Such an identification is achieved by (C.19)-(C.22).

\section{String theory embedding and CFT duals}

1. It has been shown in [190] that the conical space $\mathcal{C}$ (11.33) can be embedded as a solution of three-dimensional supergravity based on the supergroup 
$S U(1,1 \mid 2) \times S U(1,1 \mid 2)$ described by (11.3). The latter appears as the low energy description of Type IIB string theory on $S^{3} \times T^{4}$. At a first sight supersymmetrization of conical spaces seems to be an impossibility since the candidate Killing spinors 93 typically pick up phases when transported around the conical singularity, and therefore are not single valued. It was shown in 194] that this problem could be avoided by employing an extended supersymmetry $\mathcal{N}=(2,0)$ (see [195] for the notation $\mathcal{N}=(p, q)$ supergravity) and assigning a background value to the gauge field (which occurs in the supergravity multiplet). By choosing the background value appropriately the gravitational holonomy picked up by the Killing spinors can be cancelled by the gauge holonomy. Since the extended supergravity $\mathcal{N}=(2,0)$ can be embedded, further, in the $S U(1,1 \mid 2) \times S U(1,1 \mid 2)$ supergravity which is $\mathcal{N}=(4,4)$, the conical space $\mathcal{C}$ can be embedded as solutions of this latter supergravity. We therefore land up with a solution $\mathcal{C} \times S^{3} \times T^{4}$ of type IIB supergravity [190]. The background value of the $S U(2)$ gauge field is given by $A=\frac{l}{2} \gamma d \phi \tau_{3}$; from the point of IIB theory this is one of the "Kaluza-Klein" gauge fields that appear on reduction on $S^{3}$.

The CFT dual of the conical spaces (11.33), from this viewpoint, is identified [190] as the spectrally flowed CFT Hilbert space with spectral flow parameter

$$
\eta=\sqrt{\gamma}
$$

The spectral flow provides a one-parameter interpolation between the NS $(\eta=0)$ and $\mathrm{R}(\eta=1)$ sectors of the CFT, just as the conical spaces (parameterized by $\gamma$ ) interpolate between $\operatorname{AdS}_{3}(\gamma=0)$ and zero-mass BTZ $(\gamma=1)$. Note that the spectrally flowed energy formula of the CFT ground state:

$$
L_{0}|0\rangle_{\eta}=\bar{L}_{0}|0\rangle_{\eta}=-\frac{c}{24} \eta^{2}|0\rangle_{\eta} .
$$

This precisely agrees with the ADM mass of the conical spaces provided one uses (11.37). Indeed, the free energy at a finite temperature also agrees with the CFT calculation [190].

2. In the above embedding, the identification (C.19) acts apparently only on the $\mathrm{AdS}_{3}$, and not on the $S^{3} \times T^{4}$, although the vev of the $\mathrm{SU}(2)$ gauge field indirectly does affect the $S^{3}$. In [196, 197] a different, though perhaps not entirely unrelated, embedding of (11.33) into type IIB theory is used where the holonomy acts simultaneously on $\mathrm{AdS}_{3}$ as well as on $S^{3}$ (as we go around the conical singularity in $\mathrm{AdS}_{3}$, we also go around a circle in $S^{3}$ ), leading to an embedding in type IIB theory as $\left(\mathrm{AdS}_{3} \times S^{3}\right) / Z \times T^{4}$. This description naturally arise as near-horizon limit of spinning black holes. 
The CFT dual for the conical spaces in this approach are (a microcanonical ensemble of) RR states which depend on the parameter $\gamma$ [see [196], Eqns. (118)-(121)].

3. For $\gamma=1 / N^{2}$ the conical space (11.33) becomes the orbifold $\mathrm{AdS}_{3} / Z_{N}$ which has an exact world sheet CFT description and hence can be embedded as an exact solution in string theory [198]. This can be done in two ways:

- $\operatorname{AdS}_{3} / Z_{N} \times S^{3} \times K$. This theory is tachyonic, and forms a model of closed string tachyons.

- $\left(\mathrm{AdS}_{3} \times S^{3}\right) / Z_{N} \times K$. This theory is supersymmetric and has no tachyons. The $\mathrm{AdS}_{3} / \mathrm{CFT}_{2}$ dual of the conical defect from this viewpoint is given in terms of a fractionally moded $\mathcal{N}=(4,4)$ superconformal algebra, the fractional moding being given by $1 / N$ spectral flow [198] from the Ramond sector.

\subsubsection{Black hole creation by particle collision}

In 3D gravity $(\Lambda<0)$ there are explicit solutions [199] where a BTZ black hole is created by point particle collision. We will very briefly mention some salient points here:

- The conical spaces (11.33) above are a special case of the conical spacetimes for a moving point particle. We will describe here the simplest case of a moving particle which turns out be that of a massless particle. In this case, the geodesic g (11.34) is specified by the "meomenta"

$$
p^{0}=p^{1}=\tan \epsilon, p^{2}=0 .
$$

In coordinates (C.9) g now satisfies

$$
\phi(t)=0, \rho(t)=\tan (t / 2) .
$$

The gravitational back-reaction of such a particle can be exactly computed and the resulting spacetime is obtained by cutting out a wedge $W$ from $\mathrm{AdS}_{3}$, with edges $\partial W=w_{+} \cup w_{-}$,

$$
w_{ \pm}: \frac{2 \rho}{1+\rho^{2}} \sin (\epsilon \pm \phi)=\sin t \sin \epsilon
$$

where $w_{+}$are $w_{-}$are identified. The identification is achieved by quotienting $\mathrm{AdS}_{3}$ by an isometry as in Eqns. (C.19) and (C.20) with the momenta now given by (11.38). It is clear that the geodesic $\mathrm{g}$ (11.39) is a fixed point set of the quotienting operation (C.19). Note that under this identification $w_{+} \equiv w_{-}=\mathrm{g}$. Thus a moving particle is described by a wedge $W$ as 
constructed above. The spacetime constructed this way is $\mathrm{AdS}_{3} / Z$, where the $Z$ is a discrete subgroup of the isometry group as just described. Hence the resulting spacetime remains an exact solution of three-dimensional gravity with $\Lambda<0$. The energy of the particle is related to the parameter $\epsilon$ which determines the holonomy of the conical spacetime.

- It is easy to generalize the above procedure to construct spacetimes representing two particles moving towards each other. Each geodesic, $\mathrm{g}^{(1)}$ or $\mathrm{g}^{(2)}$, represents a wedge, $W^{(1)}$ or $W^{(2)}$, with edges given by

$$
\begin{aligned}
& w_{ \pm}^{(1)}: \frac{2 \rho}{1+\rho^{2}} \sin (\epsilon \pm \phi)=\sin t \sin \epsilon \\
& w_{ \pm}^{(2)}: \frac{2 \rho}{1+\rho^{2}} \sin (\epsilon \pm \phi)=-\sin t \sin \epsilon
\end{aligned}
$$

Like before $w_{+}^{(1)} \equiv w_{-}^{(1)} \equiv \mathrm{g}^{(1)}$ under the holonomy matrix $\mathbf{u}^{(1)}=\mathbf{1}+$ $\tan \epsilon\left(\gamma_{0}+\gamma_{1}\right)$ and similarly for the second particle. The full spacetime is represented in terms of two charts, one obtained by quotienting with the holonomy matrix $\mathbf{u}^{(1)} \mathbf{u}^{(2)}$, and the other one by quotienting with $\mathbf{u}^{(2)} \mathbf{u}^{(1)}$. Once again, the full spacetime is $\mathrm{AdS}_{3} / Z$, where the quotienting is by a discrete isometry subgroup; hence the resulting spacetime is an exact solution of $\mathrm{AdS}_{3}$-gravity.

- Since the above construction gives the full spacetime, the time-development of the collision process is computed by looking at the Poincare discs (see definition near (C.9)) at various times $t$. Thus, on the Poincare disc corresponding to $t=0$, the two wedges $W^{(1)}$ and $W^{(2)}$ meet; this, therefore represents the time when the two massless particles collide. At later times $t>0$, the Poincare discs exhibit a single wedge (the two wedges get identified!) which corresponds to the worldline

$$
\frac{2 \rho}{1+\rho^{2}}=\sin (t) \tan (\epsilon)
$$

For energies $\epsilon$ low enough so that $\pi / 4>\epsilon>0, \tan (\epsilon)<1$. It is easy to verify that (11.40) then represents a timelike worldline. In this energy range, therefore, the collision of two massless particles results in a single massive particle (see Figure 5, [199]). For higher energies $\pi / 2>\epsilon>\pi / 4$, i.e. $\tan (\epsilon)>1$, hence the geodesic (11.40) is spacelike. As shown in [199], this spacelike worldline is identified with the future singularity of a BTZ black hole (see Figure 6, [199]). Indeed the holonomy matrix is identified as exactly the one appropriate for a BTZ black hole.

The spacetime constructed this way therefore corresponds to formation of a BTZ black hole by a collision of two particles. Once again, since the identifi- 
cations used correspond to discrete isometries, we have an exact solution of $\mathrm{AdS}_{3}$ gravity.

The embedding of the above solutions in string theory or $\mathrm{AdS}_{3}$ supergravity remains an open problem, although it is likely that the constructions described here will admit straightforward generalizations. A more interesting problem is to understand the CFT dual of these solutions. If we succeed in applying any one of the candidate CFT duals of conical spaces as described above, we will have a unitary quantum mechanical description of black hole formation.

We should mention that black hole formation in three dimensions can also be described as a collapsing scalar field confiuration [200] or as collpsing dust shells [201]. The former process, which exhibits a critical scaling behaviour, has been discussed in the context of $\mathrm{AdS}_{3} / \mathrm{CFT}_{2}$ correspondence in [202]. CFT duals of collapsing dust shell solutions in AdS spaces are discussed in detail in [203, 204, 205. 


\section{Concluding remarks and open problems}

In this Report (see Section 1.4 for a more detailed summary) we have presented a detailed calculable formalism of the near extremal black hole (Sections 2,3) of type IIB string theory in terms of the D1-D5 system of branes (Sections 4,5). We discussed (Section 8) how for this black hole the thermodynamics and also the rates of Hawking radiation of all the massless particles can be reproduced from string theory to match the results derived in supergravity. The facility of extrapolating weak coupling calculations to the strong coupling regime owes to the high degree of supersymmetry that exists in the effective Lagrangian of the low energy degrees of freedom in the string theory (Sections 7,9). As we emphasized in this Report, a crucial input (Section 6) in the calculation of Hawking rates comes from the AdS/CFT correspondence of Maldacena 42] without which the Hawking radiation from some massless scalars is impossible to calculate from CFT (in particular, we saw in Sections 8.7 and 8.6.1 that we get incorrect results if we use the earlier DBI approach to derive the interaction of the D1-D5 bound state with the Hawking quanta). We also presented a review of $\mathrm{AdS}_{3} / \mathrm{CFT}_{2}$ correspondence beyond the supergravity approximation (using the NS5 version, see Section 10), and some applications of this correspondence for black hole formation in three dimensions (Section 11) by thermal transition and by collision of point particles.

In the light of what is achieved the following open problems naturally suggest themselves:

1. The emergence of $A d S_{3}\left(\times S^{3}\right)$ spacetime from the the $\mathcal{N}=(4,4) \mathrm{SCFT}$ is an open important open problem. In the context of $A d S_{5} \times S^{5}$ Dorey et al 206] (see also [207]) have argued that this spacetime emerges from the moduli and the fermion zero modes associated with the large $\mathrm{N}$ saddle point of the dual $\mathcal{N}=4$ SUSY gauge theory. Another approach to the question of how the radial direction of the AdS space arises from the viewpoint of the boundary theory is via Liouville theory where the Liouville or conformal degree of freedom is interpreted as a space dimension [208, 209, 210, 211]. For a recent discussion of formulation of holography in $A d S_{3}$ using Liouville theory see [212, 213]. Also see [214] for a discussion of black hole creation by point particles using Liouville theory.

2. Although the D1-D5 system provides a correct derivation of the black hole entropy, there is no precise understanding of why the entropy is given by the area of the event horizon. The reason why we lack this understanding is that the counting of microstates (see Section 8) is peformed in the dual theory. An important question to answer is to understand these states in the language of supergravity/string theory. Related questions have been discussed in [215, 216]. See also [217] where a conformal algebra is constructed in $\mathrm{AdS}_{3}$ 
supergravity with a central charge that coincides with that of the boundary CFT obtained from the D1-D5 system.

3. A matrix string description of the D1-D5 black hole was proposed in [218]. It will be interesting to make a detailed comparison between this work and the microscopic formulation presented in this Report.

4. Most of the discussion (especially where it involves the micro-description) in the present Report involves BPS or near-BPS black holes. There is a vast amount of literature on nonsupersymmetric black holes in supergravity, but we are quite far from a microscopic understanding of them. Works which address this problem include

- D0 brane blackholes (see [219] and references therein): Quantum mechanics of $N$ D0 branes at a finite temperature is analysed at large 'tHooft coupling $g_{s} N$, using a mean-field theory approximation. This leads to an entropy in good agreement (over a cetain range of temperatures) with the Bekenstein-Hawking entropy of a ten dimensional type IIA black hole carrying 0-brane charge.

- Correspondence principle and string-black hole phase transitions (see also Section 11.3): It has been shown [34, 35] that the entropy formula for a large class of nonsupersymmetric black holes agrees with that for a highly excited string state (with the same mass and charge) at a correspondence point where the curvature at the horizon becomes of the order of the string scale. This suggests a phase transition 36, 37] from a string state to a black hole (see also 220, 221]). A precise understanding of such a transition is obviously important.

- Closed string tachyons and black hole entropy [222]: A string theoretic version is suggested of the Gibbons-Hawking derivation [28] of entropy of a Schwarzschild black hole. The Schwarzschild black hole is in fact replaced by a cone for computing the entropy. The corresponding orbifold string theory has a closed string tachyon whose dynamics is dealt with using the techniques of [223]. It is obvious interest to understand this calculation in terms of miscrostates.

5. One of the potential applications of the AdS/CFT correspondence (especially in the conext of $\mathrm{AdS}_{3}$ ) is black hole dynamics. In Section 11.2 we briefly discussed the problem of black hole formation by particle collisions in $\mathrm{AdS}_{3}$ (see also [202] which discusses Choptuik scaling 200] in the context of $\mathrm{AdS}_{3} / \mathrm{CFT}_{2}$ correspondence). Clearly, the problem of evaporation of black holes too maps into interesting time dependent phenomena in the dual gauge 
theory/CFT. The Euclidean Ads/CFT correspondence describes equilibrium physics; the approach to this equilibrium is an important unsolved problem.

6. Issues of singularities and the D1-D5 system: The D1-D5 system (with K3 compactification) has been used to resolve naked singularities [13]. The detailed understanding of this mechanism is an important problem, especially in what precise sense the gauge theory acts as a source for the geometry, and how precisely the "matching" of the gauge theory and the geometry can be understood.

7. Recently [224] a particular scaling limit of AdS spaces ("pp wave") is found where string theory in the Ramond-Ramond background is solved exactly, leading to new insights into the AdS/CFT correspondence. It is important to explore the consequences for the D1-D5 system, in particular to understand from the CFT the IIB string spectrum in the pp-wave limit of $A d S_{3} \times S^{3}$ 224, 225.

8. We found in Section 2.6.3 that the two dimensional black hole 94, 95, 96 arises as a limit of the non-extremal 5-brane. In [226] a holographic description of the two dimensional black hole is proposed in terms of a quantum mechanical matrix model. It will be interesting to see how this matrix model fits into (a holographic description of) the five-brane theory, e.g. whether it can perhaps lead to the phenomenological model of [227] consisting of a gas of strings on the five brane (with tension $1 /\left(2 \pi Q_{5} g_{s} \alpha^{\prime}\right)$ and central charge $=6)$, that gives the Bekenstein-Hawking entropy of this black hole $S=U_{0}^{2} g_{\mathrm{YM}} \sqrt{Q_{5}}$. It will be interesting to compare both these approaches with the recent calculation [228] of the partition function of the two-dimensional black hole.

\section{Acknowledgments}

We would like to thank L. Alvarez-Gaume, S. Das, T. Damour, A. Dhar, M.R. Douglas, D. Gross, S.F. Hassan, K. Krasnov, K. Maeda, J. Maldacena, G. Moore, K.S. Narain, A. Sen, L. Susskind, G. Veneziano and E. Witten for useful discussions. The work of J.R.D is supported by NSF grant PHY00-98396. 


\section{A. Euclidean derivation of Hawking temperature}

We will present a derivation (the purist may regard this as a "mnemonic for a derivation", for more detailed accounts see, e.g. [7, 1, 28) of Hawking temperature for a class of black holes represented by the metric

$$
d s^{2}=-F(r) C(r) d t^{2}+d r^{2} / C(r)+H(r) r^{2} d \Omega^{2}
$$

which of course include (1.2) and (1.3). The black hole could be three, four or higher dimensional, the number of angles represented by $d \Omega^{2}$ varying accordingly.

We will assume that $C(r)$ vanishes at the real value $r=r_{h}$ and is non-vanishing for $r>r_{h}\left(C(r)=0\right.$ can have smaller roots than $r_{h}$; they are irrelevant for the present discussion). We will also assume that $F(r)$ and $H(r)$ are smooth and positive for $r \geq r_{h}$. For asymptotically flat black holes, $C(r), F(r), H(r) \rightarrow 1$ as $r \rightarrow \infty . r=r_{h}$ will correspond to the location of the event horizon.

We will focus our attention near $r=r_{h}$, where

$$
C(r)=C^{\prime}\left(r_{h}\right)\left(r-r_{h}\right)+O\left(r-r_{h}\right)^{2}
$$

It is useful to define a new radial coordinate $\rho$ :

$$
\left.\left.\left.\left.d \rho^{2}=d r^{2} / C(r), r \in\right] r_{h}, \infty\right], \rho \in\right] 0, \infty\right]
$$

In the near-horizon region (A.2)

$$
\rho=2 \sqrt{\frac{r-r_{h}}{C^{\prime}\left(r_{h}\right)}}\left[1+O\left(r-r_{h}\right)\right]
$$

The near-horizon metric is given by

$$
d s^{2}=-d t^{2} F\left(r_{h}\right)\left[C^{\prime}\left(r_{h}\right)\right]^{2} \frac{\rho^{2}}{4}+d \rho^{2}+H\left(r_{h}\right) r_{h}^{2} d \Omega^{2}
$$

The Euclidean continuation $t=-i \tau$ is given by

$$
d s_{E}^{2}=d \tau^{2} F\left(r_{h}\right)\left[C^{\prime}\left(r_{h}\right)\right]^{2} \frac{\rho^{2}}{4}+d \rho^{2}+r_{h}^{2} d \Omega^{2}
$$

If we require the $\tau, \rho$ plane not to have a conical singularity [28], we must assign the following periodicity

$$
\tau \equiv \tau+\beta, \beta=\frac{4 \pi}{C^{\prime}\left(r_{h}\right) \sqrt{F\left(r_{h}\right)}}
$$

To see this, write $\tau=(\beta / 2 \pi) \varphi$, so that

$$
d s_{E}^{2}=d \varphi^{2} \rho^{2}\left[\frac{\beta C^{\prime}\left(r_{h}\right) \sqrt{F\left(r_{h}\right)}}{4 \pi}\right]^{2}+d \rho^{2}+H\left(r_{h}\right) r_{h}^{2} d \Omega^{2}
$$


Absence of conical singularity implies that the quantity inside the square brackets must be equal to one.

A periodic Euclidean time with period $\beta$ implies a temperature

$$
T_{H}=\hbar / \beta=\frac{\hbar C^{\prime}\left(r_{h}\right) \sqrt{F\left(r_{h}\right)}}{4 \pi}
$$

- Example 1: for the RN black hole (1.3), $r_{h}=r_{+}, C(r)=\left(1-r_{-} / r\right)(1-$ $\left.r_{+} / r\right), F(r)=1$, hence $C^{\prime}\left(r_{h}\right)=\left(r_{+}-r_{-}\right) / r_{+}^{2}$, leading to (1.7).

- Example 2: for the non-extremal five-dimensional black hole (2.40), $r_{h}=$ $r_{0}, C(r)=h f^{-1 / 3}, F(r)=f^{-1 / 3}, H(r)=f^{1 / 3}$, hence

$$
C^{\prime}\left(r_{h}\right) \sqrt{F\left(r_{h}\right)}=h^{\prime}\left(r_{0}\right) f\left(r_{0}\right)^{-1 / 2}=2 r_{0}^{-1}\left(\cosh \left(\alpha_{1}\right) \cosh \left(\alpha_{5}\right) \cosh \left(\alpha_{n}\right)\right)^{-1},
$$

leading to (2.45).

\section{Entropy and the first law}

We note that the entropy (1.10), namely

$$
S=\frac{\pi r_{h}^{2}}{G_{N} \hbar}
$$

together with (A.9), satisfies the first law of thermodynamics (1.9). E.g. for the RN black hole (1.3), consider two neighbouring states, differing in the value of $r_{+}$ (but having the same value of $\left.r_{-}\right)$. Using $\Phi=A_{0}\left(r_{h}\right)=Q / r_{+}$, we get

$$
\begin{aligned}
\Phi d Q & =\frac{1}{r_{+}} Q d Q=\frac{r_{-}}{2 G_{N} r_{+}} d r_{+} \\
T_{H} d S & =\frac{r_{+}-r_{-}}{4 \pi r_{+}^{2}} d \frac{\pi r_{+}^{2}}{G_{N}}=\frac{r_{+}-r_{-}}{2 G_{N} r_{+}} \\
d M & =d \frac{r_{+}+r_{-}}{2 G_{N}}=\frac{1}{2 G_{N}} d r_{+}=T_{H} d S+\Phi d Q
\end{aligned}
$$

In the first line we have used $Q^{2}=r_{+} r_{-} / G_{N} \Rightarrow Q d Q=\frac{1}{2} d\left(Q^{2}\right)=\frac{r_{-}}{2 G_{N}} d r_{+}$.

We thus verify Eqn. (1.9). 


\section{B. A heuristic motivation for Rules 1 and 2 in Section (2.4)}

We present a brief, heuristic, motivation for the algorithm of section 2.4. Suppose we view a static Schwarzschild black hole, of ADM mass $m$, from the viewpoint of the five-dimensional $\left(R^{4} \times S^{1}\right)$ Kaluza-Klein theory (we use coordinates $x^{0,1,2,3}$ for $R^{4}$ and $x^{5}$ for the $\left.S^{1}\right)$. The 5 -momentum $\left(p_{0}, \vec{p}, p_{5}\right)$ would be given by

$$
p_{0}=m, \quad p_{5} \propto \text { charge }=0, \quad \vec{p}=0
$$

The $p_{5}$ equation follows because the Schwarzschild black hole is neutral.

A way of generating charged solutions (see, e.g., [229, 230, 231] for details) is to unwrap the above solution in five non-compact dimensions, perform a boost in the 0-5 plane (which is a symmetry of the non-compact theory), and compactify the new $x_{5}^{\prime}$ direction to get a charged (RN) black hole in four non-compact dimensions. The momenta should transform as

$$
p_{0}^{\prime} \equiv M=m \cosh \delta, \quad p_{5}^{\prime} \equiv Q=m \sinh \delta, \quad \vec{p}^{\prime}=0
$$

In the above, we have absorbed the factor of radius in the definition of the charge so that the extremality condition reads $M=Q$. The extremal limit can be attained by

$$
\delta \rightarrow \infty, m \rightarrow 0, m e^{\delta} \rightarrow \text { constant }
$$

so that

$$
Q \rightarrow M=m e^{\delta} / 2, \quad p_{R}^{\prime} \equiv p_{0}^{\prime}-p_{5}^{\prime} \rightarrow 0
$$

Near-extremal limit

The near-extremal limit is obtained by keeping the leading corrections in the expansion parameter $e^{-\delta}$. Thus,

$$
\tilde{Q} / M=\tanh \delta \simeq 1-m^{2} /\left(2 \tilde{Q}^{2}\right), \quad p_{R}^{\prime} \ll p_{L}^{\prime}, p_{L}^{\prime} \equiv p_{0}^{\prime}+p_{5}^{\prime}
$$

In terms of these parameters, the four-dimensional metric for a near-extremal charged (RN) black hole is given by

$$
\begin{aligned}
d s_{4}^{2} & =-f d t^{2}+f^{-1} d r^{2}+r^{2} d \Omega_{2}^{2} \\
f & \equiv 1-2 M / r+\tilde{Q}^{2} / r^{2}=f_{\text {ext }} h(r) \\
f_{\text {ext }} & \equiv(1-\tilde{Q} / r)^{2}, \quad h(r)=(1-\mu / r) \\
\mu & =m^{2} / \tilde{Q}
\end{aligned}
$$

The last equality implies

$$
\tilde{Q}=\mu \sinh ^{2} \delta
$$

same as (2.36) above. Also, the second equality agrees with Rule 1 for relating the non-extremal $g_{t t}, g_{r r}$ to their extremal counterparts. 
Of course, we have considered here only the near-extremal case. The remarkable thing about the algorithm mentioned in Section 2.4 is that it works for arbitrary deviations from extremality. 


\section{Coordinate systems for $\mathrm{AdS}_{3}$ and related spaces}

For a more detailed discussion and derivations, see, e.g. [10, 232]. This Appendix is meant to serve as a compendium of some definitions and results about the geometry of $\mathrm{AdS}_{3}$ and related spaces. We discuss both Lorentzian and Euclidean signatures.

\section{C.1 $\operatorname{AdS}_{3}$}

$\mathrm{AdS}_{3}$ is defined as a hyperboloid

$$
-Y_{0}^{2}-Y_{-1}^{2}+Y_{1}^{2}+Y_{2}^{2} \equiv Y_{+} Y_{-}+\sum_{\mu=0,1} Y_{\mu} Y^{\mu}=-l^{2}
$$

in $R^{2,2}$ with metric

$$
d s^{2}=-d Y_{0}^{2}-d Y_{-1}^{2}+d Y_{1}^{2}+d Y_{2}^{2}=d Y_{\mu} d Y^{\mu}+d Y_{+} d Y_{-}
$$

Here $Y_{ \pm}=Y_{2} \pm Y_{-1}$. The condition (C.1) can be equivalently stated by saying that a point in $\mathrm{AdS}_{3}$ is represented by an $\mathrm{SL}(2)$ matrix $(1 / l) \mathbf{Y}$ where

$$
\mathbf{Y}=Y_{-1} \mathbf{1}+Y^{a} \gamma_{a}, a=0,1,2
$$

where

$$
\gamma_{0}=\left(\begin{array}{cc}
0 & 1 \\
-1 & 0
\end{array}\right), \gamma_{1}=\left(\begin{array}{ll}
0 & 1 \\
1 & 0
\end{array}\right), \gamma_{2}=\left(\begin{array}{cc}
1 & 0 \\
0 & -1
\end{array}\right) .
$$

\section{Global coordinates:}

$$
\begin{aligned}
& Y_{-1}=l \cosh \mu \sin t ; \quad Y_{0}=l \cosh \mu \cos t \\
& Y_{1}=l \sinh \mu \cos \phi ; \quad Y_{2}=l \sinh \mu \sin \phi
\end{aligned}
$$

The metric is

$$
d s^{2}=l^{2}\left(-\cosh ^{2} \mu d t^{2}+d \mu^{2}+\sinh ^{2} \mu d \phi^{2}\right)
$$

By redefining $\sinh \mu=r / l$ we get

$$
d s^{2}=l^{2}\left[-\left(1+\frac{r^{2}}{l^{2}}\right) d t^{2}+\frac{d r^{2}}{1+\frac{r^{2}}{l^{2}}}+\frac{r^{2}}{l^{2}} d \phi^{2}\right]
$$

Range of coordinates: $\phi \in[0,2 \pi], \mu$ (equivalently $r) \in(0, \infty), t \in[0,2 \pi]$

If we unwrap $t$ to the range $t \in(-\infty, \infty)$ we get the so-called $\mathrm{CAdS}_{3}$ (covering space) which is geodesically complete.

There is another popular form, given by the coordinate transformation $\rho=$ $\tanh (\mu / 2)$, leading to

$$
d s^{2}=-\left(\frac{1+\rho^{2}}{1-\rho^{2}}\right)^{2} d t^{2}+\left(\frac{2}{1-\rho^{2}}\right)^{2}\left(d \rho^{2}+\rho^{2} d \phi^{2}\right)
$$


The section at any given $t$ has the metric of a disc, called the Poincare disc.

Poincare coordinates:

$$
Y_{+}=l^{2} / u, \quad Y_{\mu}=l x_{\mu} / u
$$

$\left(Y_{-}\right.$determined by $($C.1 $\left.)\right)$. The metric is

$$
d s^{2}=\frac{l^{2}}{u^{2}}\left(d u^{2}+d x^{\mu} d x_{\mu}\right)
$$

Range: $u \in(0, \infty), x^{0}=t \in(-\infty, \infty), x^{1} \in(-\infty, \infty)$ : covers a half of $\operatorname{AdS}_{3}$ (C.1).

\section{C.2 BTZ black hole}

The three-dimensional black hole [92, 232] in an asymptotically AdS spacetime $\left(\Lambda:=-1 / l^{2}\right)$, of mass $M$ and angular momentum $J$, is given by the metric

$$
\begin{aligned}
d s^{2} & =-N^{2}(r) d t^{2}+d r^{2} / N^{2}(r)+r^{2}\left(N_{\phi}(r) d t+d \phi\right)^{2} \\
N^{2}(r) & =\frac{r^{2}}{l^{2}}-M+\left(\frac{J}{2 r}\right)^{2}, \quad N_{\phi}(r)=\frac{J}{2 r^{2}} \\
\text { Range : } & t \in(-\infty, \infty), r \in(0, \infty), \phi \in[0,2 \pi] .
\end{aligned}
$$

Properties:

- The lapse function $N(r)$ vanishes at $r=r_{ \pm}$where

$$
r_{ \pm}=l\left[\frac{M}{2}\left(1 \pm \sqrt{1-\left(\frac{J}{M l}\right)^{2}}\right)\right]^{1 / 2}
$$

Thus,

$$
N^{2}(r)=\frac{1}{l^{2} r^{2}}\left(r^{2}-r_{+}^{2}\right)\left(r^{2}-r_{-}^{2}\right), \quad N_{\phi}(r)=\frac{r_{+} r_{-}}{r^{2} l} .
$$

$r_{+}$corresponds to the event horizon.

- $g_{00}=-N(r)^{2}+r^{2} N_{\phi}(r)^{2}=\frac{r^{2}}{l^{2}}-M$ vanishes at

$$
r_{\text {erg }}=l \sqrt{M}
$$

which represents the surface of infinite red-shift.

- These three special values of $r$ satisfy $r_{-} \leq r_{+} \leq r_{\text {erg. }}$. The region between $r_{+}$and $r_{\text {erg }}$ is called the "ergosphere".

Furthermore, the above black hole (C.11) can be obtained as a quotient of the $\mathrm{AdS}_{3}$ space (C.1). We show this for the $J=0, M>0 \mathrm{BTZ}$, given by

$$
d s^{2}=-\left[\frac{r^{2}}{l^{2}}-M\right] d t^{2}+\left[\frac{r^{2}}{l^{2}}-M\right]^{-1} d r^{2}+r^{2} d \phi^{2}
$$


In the patch $Y_{-1}^{2} \geq Y_{1}^{2}, Y_{0}^{2} \leq Y_{2}^{2}$ we define

$$
\begin{aligned}
Y_{-1} & = \pm \frac{r}{\sqrt{M}} \cosh \phi \sqrt{M}, \quad Y_{0}=\left(\frac{r^{2}}{M}-l^{2}\right)^{1 / 2} \sinh \frac{t \sqrt{M}}{l} \\
Y_{1} & =\frac{r}{\sqrt{M}} \sinh \phi \sqrt{M}, \quad Y_{2}= \pm\left(\frac{r^{2}}{M}-l^{2}\right)^{1 / 2} \cosh \frac{t \sqrt{M}}{l}
\end{aligned}
$$

With this, the metric (C.2) coincides with (C.14). In (C.14) the angle $\phi \equiv \phi+2 \pi$. This implies, through (C.15) a discrete quotienting of the original hyperboloid. We thus find that the BTZ metric is equivalent to $\mathrm{AdS}_{3} / Z$. (We have shown this here in a certain coordinate patch, but it can be proved more generally [232].)

\section{C.3 Conical spaces}

$$
d s^{2}=l^{2}\left[-\left(\gamma+\frac{r^{2}}{l^{2}}\right) d t^{2}+\frac{d r^{2}}{\gamma+\frac{r^{2}}{l^{2}}}+r^{2} d \phi^{2}\right]
$$

Range: $r \in(0, \infty), t \in(-\infty, \infty), \phi \in[0,2 \pi]$.

Define $r=\sqrt{\gamma} \tilde{r}, t=\tilde{t} / \sqrt{\gamma}, \phi=\tilde{\phi} / \sqrt{\gamma}$, get

$$
d s^{2}=l^{2}\left[-\left(1+\frac{\tilde{r}^{2}}{l^{2}}\right) d \tilde{t}^{2}+\frac{d \tilde{r}^{2}}{1+\frac{\tilde{r}^{2}}{l^{2}}}+\tilde{r}^{2} d \tilde{\phi}^{2}\right]
$$

$$
\text { Range: } \tilde{\phi} \in[0,2 \pi \sqrt{\gamma}] \text {. }
$$

Defect angle $\Delta=2 \pi(1-\sqrt{\gamma})$.

\section{Conic as $\mathrm{AdS}_{3} / Z$}

We will show that (C.17) can be obtained from (C.1), C.2 modulo the following identification (using the notation of (C.3))

$$
\mathbf{Y} \equiv \mathbf{u}^{-1} \mathbf{Y u}
$$

The holonomy matrix $\mathbf{u}$ is given by the momentum $p^{a}, a=0,1,2$ of the particle:

$$
\mathbf{u}=u \mathbf{1}+p^{a} \gamma_{a}
$$

In case of a static particle, of mass $m$

$$
p^{0}=m, p^{1}=p^{2}=0 .
$$

Hence $\mathbf{u}=u \mathbf{1}+m \gamma_{0}$, where $u=\sqrt{1-m^{2}}$ by the SL(2) condition. The identification (C.19), affects only the components $Y_{1}, Y_{2}$ and reads

$$
\left(\begin{array}{l}
Y_{1} \\
Y_{2}
\end{array}\right) \equiv\left(\begin{array}{cc}
1-2 m^{2} & -2 m \sqrt{1-m^{2}} \\
2 m \sqrt{1-m^{2}} & 1-2 m^{2}
\end{array}\right)\left(\begin{array}{l}
Y_{1} \\
Y_{2}
\end{array}\right)
$$


Note that the matrix is an $S O(2)$ rotation matrix with $\cos (\alpha)=1-2 m^{2}$. In terms of the coordinates (C.5), C.17) this implies an identification

$$
\tilde{\phi} \equiv \tilde{\phi}+\cos ^{-1}\left(1-2 m^{2}\right)
$$

Comparing with $(\overline{\mathrm{C} .18})$ we get a relation between the parameter $\gamma$ and the mass $m$

$$
1-2 m^{2}=\cos (2 \pi \sqrt{\gamma})
$$

\section{C.4 Euclidean sections and Thermal Physics}

Euclidean $\mathrm{AdS}_{3}$ can be defined by the global coordinates of (C.7) with the replacement $\mathrm{t}=-i t$, leading to the metric

$$
d s^{2}=l^{2}\left[\left(1+\frac{r^{2}}{l^{2}}\right) d \mathrm{t}^{2}+\frac{d r^{2}}{1+\frac{r^{2}}{l^{2}}}+r^{2} d \phi^{2}\right]
$$

where (cf. (C.8)

$$
\phi \equiv \phi+2 \pi
$$

Like (C.3) in the Lorentzian case, a point in Euclidean $\mathrm{AdS}_{3}$ can be alternatively defined as a hermitian matrix $(1 / l) \mathbf{Y}$ of unit determinant (the space of such matrices is called $\mathrm{H}$ ) where

$$
\mathbf{Y}=Y_{-1} \mathbf{1}+Y^{a} \tilde{\gamma}_{a}, a=0,1,2
$$

and the new $\tilde{\gamma}$ matrices are obtained by replacing $\gamma_{0}$ in (C.4) by $\tilde{\gamma}_{0}=i \gamma_{0}$. The determinant condition now reads

$$
-Y_{-1}^{2}+Y_{0}^{2}+Y_{1}^{2}+Y_{2}^{2}=-l^{2}
$$

The metric in this parameterization is

$$
d s^{2}=\operatorname{Tr} d \mathbf{Y}^{2}=-d Y_{-1}^{2}+d Y_{0}^{2}+d Y_{1}^{2}+d Y_{2}^{2}
$$

To make contact with the global coordinates of (C.23) we first introduce the by the following parameterization of $\mathrm{H}$

$$
\mathbf{Y} / l=\left(\begin{array}{cc}
e^{u} & 0 \\
0 & e^{-u}
\end{array}\right)\left(\begin{array}{cc}
\sqrt{1+r^{2} / l^{2}} & r / l \\
r / l & \sqrt{1+r^{2} / l^{2}}
\end{array}\right)\left(\begin{array}{cc}
e^{\bar{u}} & 0 \\
0 & e^{-\bar{u}}
\end{array}\right)
$$

In order to cover $\mathrm{H}$ only once, we must identify

$$
2 u \equiv 2 u+i 2 n \pi
$$

The metric (C.27) then becomes

$$
d s^{2}=l^{2}\left[\frac{(d u+d \bar{u})^{2}}{1+r^{2} / l^{2}}+\frac{d r^{2}}{1+r^{2} / l^{2}}-\frac{r^{2}}{l^{2}}(d u-d \bar{u})^{2}\right]
$$


A comparison of the peroidicities (C.29) and (C.24) suggests that we define

$$
2 u=\mathrm{t}+i \phi .
$$

With this definition we recover (C.23) from (C.30).

For Euclidean $\mathrm{AdS}_{3}$, the periodicity in the $t$ direction is to be supplied as an input from physics. A thermal ensemble implies in the usual fashion $t \equiv t+1 / T$. In addition to a temperature one may want to introduce an angular potential (conjugate to angular momentum $J \sim i \partial / \partial \phi$ ); such an ensemble, described by Tr $\exp [-H / T+i \Phi J]$ implies a more general (twisted) identification

$$
\phi+i \mathrm{t} \equiv \phi+i \mathrm{t}+\Phi+i / T:=\phi+i \mathrm{t}+i \beta
$$

where the second step is a definition of the "complex temperature" (cf. A.7))

$$
\beta=1 / T-i \Phi
$$

\section{Euclidean Poincare coordinates}

The Euclidean verison of (C.10) is defined by

$$
\mathbf{Y} / l=\left(\begin{array}{cc}
e^{u} & 0 \\
0 & e^{-u}
\end{array}\right)\left(\begin{array}{cc}
h+w \bar{w} / h & w / h \\
\bar{w} / h & 1 / h
\end{array}\right)
$$

The metric (C.27) becomes

$$
d s^{2}=\frac{1}{h^{2}}\left(d w d \bar{w}+d h^{2}\right)
$$

Euclidean BTZ

Euclidean BTZ is usually defined by defining $\mathrm{t}=-i t, J_{E}=i J$ in (C.11):

$$
d s^{2}=\left[\frac{r^{2}}{l^{2}}-M-\left(\frac{J_{E}}{2 r}\right)^{2}\right] d \mathrm{t}^{2}+\left[\frac{r^{2}}{l^{2}}-M-\left(\frac{J_{E}}{2 r}\right)^{2}\right]^{-1} d r^{2}+r^{2}\left[\frac{i J_{E}}{2 r^{2}} d \mathrm{t}+d \phi\right]^{2}
$$

where (cf. (C.11))

$$
\phi \equiv \phi+2 \pi
$$

Like in the Lorentzian case, we can obtain the metric (C.36) as $\mathrm{EBTZ}^{\mathrm{C}}=\mathrm{EAdS}_{3} / \mathrm{Z}$ [90, 191], as follows. Define a quotient of (C.25), by [92]

$$
\mathbf{Y} \equiv\left(\begin{array}{cc}
e^{-i \pi \tau} & 0 \\
0 & e^{i \pi \tau}
\end{array}\right) \mathbf{Y}\left(\begin{array}{cc}
e^{i \pi \bar{\tau}} & 0 \\
0 & e^{-i \pi \bar{\tau}}
\end{array}\right)
$$

In terms of (C.28) the above identification (C.38) reads

$$
2 u \equiv 2 u+2 \pi i n \tau
$$


Note that this identification is in addition to (C.29). The two identifications defines for us two independent cycles in the $u$-plane. We will show below that the description (C.36) follows by identifying the cycle (C.39) with the "space" cycle, namely (C.37). This can be easily done by defining

$$
2 u=-i \tau(\phi+i \mathrm{t})
$$

The cycle (C.29) now becomes the "time", unlike in the case of thermal $\mathrm{AdS}_{3}$, where it was "space". If we define

$$
\tau=\left|r_{-}\right|-i r_{+}
$$

and a new radial coordinate $\tilde{r}$ in terms of the $r$ of (C.28), as follows

$$
r^{2} / l^{2}=\frac{\tilde{r}^{2} / l^{2}-\tau_{2}^{2}}{|\tau|^{2}}
$$

where

$$
r_{ \pm}^{2}=\frac{l^{2} M}{2}\left[1 \pm \sqrt{1+\frac{J_{E}^{2}}{M^{2} l^{2}}}\right]
$$

(note that $r_{-}=i\left|r_{-}\right|$is purely imaginary), then the metric on $\mathrm{H}$, for $\tilde{r} \geq r_{+}$, becomes (C.36) once we drop the tilde from $\tilde{r}$. Temperature

Note that the periodicity along the new "time" cycle ( $\overline{\text { C.29 }}$, through $(\overline{\mathrm{C} .40})$ implies the following complex periodicity (complex temperature)

$$
\begin{aligned}
\phi+i \mathrm{t} & \equiv \phi+i \mathrm{t}+i \beta_{0}, \\
\beta_{0} & =2 \pi i(1 / \tau)=\frac{2 \pi r_{+}}{r_{+}^{2}-r_{-}^{2}}+i \frac{2 \pi\left|r_{-}\right|}{r_{+}^{2}-r_{-}^{2}}
\end{aligned}
$$

The real temperature $T_{0} \equiv\left(\Re \beta_{0}\right)^{-1}$ is given by

$$
T_{0}=\frac{r_{+}^{2}-r_{-}^{2}}{2 \pi r_{+}}
$$

which agrees with the expression in [92].

\section{Entropy}

As can be derived from the partition function calculation in Section 11.1, the entropy of the BTZ black hole is given by [92]

$$
S=\frac{2 \pi r_{+}}{4 G_{N}^{(3)}}
$$

which of course agrees with the Bekenstein-Hawking formula (1.10) as well. 


\section{References}

[1] S. W. Hawking and W. Israel, "General relativity: an Einstein centenary survey,". Cambridge, United Kingdom: Univ. Press. (1979) 919p.

[2] M. B. Green, J. H. Schwarz, and E. Witten, "Superstring theory. Vol. 1: Introduction; Vol. 2: Loop amplitudes, anomalies and phenomenology,". Cambridge, United Kingdom: Univ. Press. ( 1987) 469 P. ( Cambridge Monographs On Mathematical Physics).

[3] J. Polchinski, "String theory. Vol. 1: An introduction to the bosonic string; Vol. 2; superstring theory and beyond,". Cambridge, United Kingdom: Univ. Press. (1998) $402 \mathrm{p}$.

[4] T. Yoneya, "Connection of dual models to electrodynamics and gravidynamics," Prog. Theor. Phys. 51 (1974) 1907-1920.

[5] J. Scherk and J. H. Schwarz, "Dual models and the geometry of space-time," Phys. Lett. B52 (1974) 347.

[6] C. W. Misner, K. S. Thorne, and J. A. Wheeler, "Gravitation,". Freeman, 1970, $1279 \mathrm{p}$.

[7] R. M. Wald, "General relativity,". Chicago, USA: Univ. Pr. ( 1984) 491p.

[8] R. P. Feynman, F. B. Morinigo, W. G. Wagner, and B. Hatfield (Ed.), "Feynman lectures on gravitation,". Reading, USA: Addison-Wesley (1995) 232 p. (The advanced book program).

[9] S. Weinberg, "Gravitation and cosmology: principles and applications of the general theory of relativity,". Wiley, 1972, $657 \mathrm{p}$.

[10] S. Hawking and G. Ellis, "The large scale structure of space time,". Cambridge Univ. Press, 1973, 391p.

[11] G. T. Horowitz and R. C. Myers, "The value of singularities," Gen. Rel. Grav. 27 (1995) 915-919, gr-qc/9503062.

[12] C. V. Johnson, N. Kaloper, R. R. Khuri, and R. C. Myers, "Is string theory a theory of strings?," Phys. Lett. B368 (1996) 71-77, hep-th/9509070.

[13] C. V. Johnson, A. W. Peet, and J. Polchinski, "Gauge theory and the excision of repulson singularities," Phys. Rev. D61 (2000) 086001, hep-th/9911161.

[14] S. S. Gubser, "Curvature singularities: The good, the bad, and the naked," hep-th/0002160.

[15] M. Natsuume, "The singularity problem in string theory," gr-qc/0108059. 
[16] S. W. Hawking, "Particle creation by black holes," Commun. Math. Phys. 43 (1975) 199-220.

[17] S. W. Hawking, "Breakdown of predictability in gravitational collapse," Phys. Rev. D14 (1976) 2460-2473.

[18] G. 't Hooft, "Black holes, Hawking radiation, and the information paradox," Nucl. Phys. Proc. Suppl. 43 (1995) 1-11.

[19] S. B. Giddings, "The black hole information paradox," hep-th/9508151.

[20] S. M. Carroll, "The cosmological constant," Living Rev. Rel. 4 (2001) 1, astro-ph/0004075.

[21] S. Weinberg, "The cosmological constant problems," astro-ph/0005265.

[22] E. Witten, "The cosmological constant from the viewpoint of string theory," hep-ph/0002297.

[23] A. Celotti, J. C. Miller, and D. W. Sciama, "Astrophysical evidence for the existence of black holes," astro-ph/9912186.

[24] P. T. Chrusciel, "No hair' theorems: Folklore, conjectures, results," gr-qc/9402032.

[25] J. D. Bekenstein, "Black hole hair: Twenty-five years after," gr-qc/9605059.

[26] B. Carter, "Has the black hole equilibrium problem been solved?," gr-qc/9712038.

[27] D. Marolf, "Some brane-theoretic no-hair results (and their field- theory duals)," gr-qc/9908017.

[28] G. W. Gibbons and S. W. Hawking, "Action integrals and partition functions in quantum gravity," Phys. Rev. D15 (1977) 2752-2756.

[29] J. D. Bekenstein, "Black holes and entropy," Phys. Rev. D7 (1973) 2333-2346.

[30] J. D. Bekenstein, "Generalized second law of thermodynamics in black hole physics," Phys. Rev. D9 (1974) 3292-3300.

[31] J. M. Bardeen, B. Carter, and S. W. Hawking, "The four laws of black hole mechanics," Commun. Math. Phys. 31 (1973) 161-170.

[32] S. W. Hawking, "Black holes and thermodynamics," Phys. Rev. D13 (1976) 191-197.

[33] G. 't Hooft, "The scattering matrix approach for the quantum black hole: An overview," Int. J. Mod. Phys. A11 (1996) 4623-4688, gr-qc/9607022. 
[34] L. Susskind, "Some speculations about black hole entropy in string theory," hep-th/9309145.

[35] G. T. Horowitz and J. Polchinski, "A correspondence principle for black holes and strings," Phys. Rev. D55 (1997) 6189-6197, hep-th/9612146;.

[36] G. T. Horowitz and J. Polchinski, "Self gravitating fundamental strings," Phys. Rev. D57 (1998) 2557-2563, hep-th/9707170.

[37] T. Damour and G. Veneziano, "Self-gravitating fundamental strings and black holes," Nucl. Phys. B568 (2000) 93-119, hep-th/9907030.

[38] A. Dhar, G. Mandal, and S. R. Wadia, "Absorption vs decay of black holes in string theory and T- symmetry," Phys. Lett. B388 (1996) 51-59, hep-th/9605234.

[39] S. R. Das and S. D. Mathur, "Comparing decay rates for black holes and D-branes," Nucl. Phys. B478 (1996) 561-576, hep-th/9606185.

[40] J. Maldacena and A. Strominger, "Black hole greybody factors and D-brane spectroscopy," Phys. Rev. D55 (1997) 861-870, hep-th/9609026.

[41] O. Aharony, S. S. Gubser, J. Maldacena, H. Ooguri, and Y. Oz, "Large N field theories, string theory and gravity," Phys. Rept. 323 (2000) 183-386, hep-th/9905111.

[42] J. Maldacena, "The large $N$ limit of superconformal field theories and supergravity," Adv. Theor. Math. Phys. 2 (1998) 231-252, hep-th/9711200.

[43] J. de Boer, "Six-dimensional supergravity on $S^{3} \times A d S_{3}$ and 2 d conformal field theory," Nucl. Phys. B548 (1999) 139-166, hep-th/9806104.

[44] C. G. Callan and J. M. Maldacena, "D-brane approach to black hole quantum mechanics," Nucl. Phys. B472 (1996) 591-610, hep-th/9602043.

[45] K. S. Stelle, "BPS branes in supergravity," hep-th/9803116.

[46] N. A. Obers and B. Pioline, "U-duality and M-theory," Phys. Rept. 318 (1999) 113-225, hep-th/9809039.

[47] J. P. Gauntlett, "Intersecting branes," hep-th/9705011.

[48] J. M. Maldacena, "Black holes in string theory," hep-th/9607235.

[49] M. Cvetic, "Properties of black holes in toroidally compactified string theory," Nucl. Phys. Proc. Suppl. 56B (1997) 1-10, hep-th/9701152.

[50] D. Youm, "Black holes and solitons in string theory," Phys. Rept. 316 (1999) 1-232, hep-th/9710046. 
[51] J. R. David, "String theory and black holes," hep-th/9911003.

[52] G. Mandal, "A review of the D1/D5 system and five dimensional black hole from supergravity and brane viewpoints," hep-th/0002184.

[53] S. R. Wadia, "Lectures on the microscopic modeling of the 5-dim black hole of IIB string theory and the D1/D5 system," hep-th/0006190.

[54] A. W. Peet, "The Bekenstein formula and string theory (N-brane theory)," Class. Quant. Grav. 15 (1998) 3291-3338, hep-th/9712253.

[55] K. Skenderis, "Black holes and branes in string theory," Lect. Notes Phys. 541 (2000) 325-364, hep-th/9901050.

[56] M. J. Duff, "Lectures on branes, black holes and anti-de Sitter space," hep-th/9912164.

[57] P. K. Townsend, "The eleven-dimensional supermembrane revisited," Phys. Lett. B350 (1995) 184-187, hep-th/9501068.

[58] E. Witten, "String theory dynamics in various dimensions," Nucl. Phys. B443 (1995) 85-126, hep-th/9503124.

[59] G. Papadopoulos and P. K. Townsend, "Intersecting M-branes," Phys. Lett. B380 (1996) 273-279, hep-th/9603087.

[60] A. A. Tseytlin, "Harmonic superpositions of M-branes," Nucl. Phys. B475 (1996) 149-163, hep-th/9604035.

[61] M. J. Duff and K. S. Stelle, "Multi-membrane solutions of D = 11 supergravity," Phys. Lett. B253 (1991) 113-118.

[62] E. Witten, "Anti-de Sitter space and holography," Adv. Theor. Math. Phys. 2 (1998) 253-291, hep-th/9802150.

[63] S. S. Gubser, I. R. Klebanov, and A. M. Polyakov, "Gauge theory correlators from non-critical string theory," Phys. Lett. B428 (1998) 105-114, hep-th/9802109.

[64] E. Cremmer, B. Julia, and J. Scherk, "Supergravity theory in 11 dimensions," Phys. Lett. B76 (1978) 409-412.

[65] J. A. de Azcarraga, J. P. Gauntlett, J. M. Izquierdo, and P. K. Townsend, "Topological extensions of the supersymmetry algebra for extended objects," Phys. Rev. Lett. 63 (1989) 2443.

[66] G. W. Gibbons and P. K. Townsend, "Vacuum interpolation in supergravity via super p-branes," Phys. Rev. Lett. 71 (1993) 3754-3757, hep-th/9307049.

[67] S. Surya and D. Marolf, "Localized branes and black holes," Phys. Rev. D58 (1998) 124013, hep-th/9805121. 
[68] G. T. Horowitz and A. Strominger, "Black strings and p-branes," Nucl. Phys. B360 (1991) 197-209.

[69] S. F. Hassan, "T-duality, space-time spinors and R-R fields in curved backgrounds," Nucl. Phys. B568 (2000) 145-161, hep-th/9907152.

[70] R. Gregory and R. Laflamme, "Black strings and p-branes are unstable," Phys. Rev. Lett. 70 (1993) 2837-2840, hep-th/9301052.

[71] A. Strominger and C. Vafa, "Microscopic origin of the Bekenstein-Hawking entropy," Phys. Lett. B379 (1996) 99-104, hep-th/9601029.

[72] D. Garfinkle and T. Vachaspati, "Cosmic string traveling waves," Phys. Rev. D42 (1990) 1960-1963.

[73] M. S. Bremer, H. Lu, C. N. Pope, and K. S. Stelle, "Dirac quantisation conditions and Kaluza-Klein reduction," Nucl. Phys. B529 (1998) 259-294, hep-th/9710244.

[74] M. Cvetic and A. A. Tseytlin, "Non-extreme black holes from non-extreme intersecting M-branes," Nucl. Phys. B478 (1996) 181-198, hep-th/9606033.

[75] G. T. Horowitz, J. M. Maldacena, and A. Strominger, "Nonextremal black hole microstates and U-duality," Phys. Lett. B383 (1996) 151-159, hep-th/9603109.

[76] A. Sen, "Strong coupling dynamics of branes from M-theory," JHEP 10 (1997) 002, hep-th/9708002.

[77] E. Eyras and S. Panda, "The spacetime life of a non-BPS D-particle," Nucl. Phys. B584 (2000) 251-283, hep-th/0003033.

[78] Y. Lozano, "Non-BPS D-brane solutions in six dimensional orbifolds," Phys. Lett. B487 (2000) 180-186, hep-th/0003226.

[79] P. Brax, G. Mandal, and Y. Oz, "Supergravity description of non-BPS branes," Phys. Rev. D63 (2001) 064008, hep-th/0005242.

[80] M. Bertolini et al., "Is a classical description of stable non-BPS D-branes possible?," Nucl. Phys. B590 (2000) 471-503, hep-th/0007097.

[81] P. Bain, "Taming the supergravity description of non-BPS D-branes: The D/anti-D solution," JHEP 04 (2001) 014, hep-th/0012211.

[82] K. A. Intriligator, M. Kleban, and J. Kumar, "Comments on unstable branes," JHEP 02 (2001) 023, hep-th/0101010.

[83] Y. C. Liang and E. Teo, "Black diholes with unbalanced magnetic charges," Phys. Rev. D64 (2001) 024019, hep-th/0101221. 
[84] R. Emparan and E. Teo, "Macroscopic and microscopic description of black diholes," Nucl. Phys. B610 (2001) 190-214, hep-th/0104206.

[85] S. H. S. Alexander, "Inflation from D - anti-D brane annihilation," Phys. Rev. D65 (2002) 023507, hep-th/0105032.

[86] G. L. Alberghi, E. Caceres, K. Goldstein, and D. A. Lowe, "Stacking non-BPS D-branes," Phys. Lett. B520 (2001) 361-366, hep-th/0105205.

[87] J. M. Maldacena and J. G. Russo, "Large N limit of non-commutative gauge theories," JHEP 09 (1999) 025, hep-th/9908134.

[88] A. Dhar, G. Mandal, S. R. Wadia, and K. P. Yogendran, "D1/D5 system with B-field, noncommutative geometry and the CFT of the Higgs branch," Nucl. Phys. B575 (2000) 177-194, hep-th/9910194.

[89] S. Ferrara, G. W. Gibbons, and R. Kallosh, "Black holes and critical points in moduli space," Nucl. Phys. B500 (1997) 75-93, hep-th/9702103.

[90] J. Maldacena and A. Strominger, " $A d S_{3}$ black holes and a stringy exclusion principle," JHEP 12 (1998) 005, hep-th/9804085.

[91] K. Sfetsos and K. Skenderis, "Microscopic derivation of the bekenstein-hawking entropy formula for non-extremal black holes," Nucl. Phys. B517 (1998) 179-204, hep-th/9711138.

[92] M. Banados, C. Teitelboim, and J. Zanelli, "The black hole in three-dimensional space-time," Phys. Rev. Lett. 69 (1992) 1849-1851, hep-th/9204099.

[93] O. Coussaert and M. Henneaux, "Supersymmetry of the $(2+1)$ black holes," Phys. Rev. Lett. 72 (1994) 183-186, hep-th/9310194.

[94] G. Mandal, A. M. Sengupta, and S. R. Wadia, "Classical solutions of two-dimensional string theory," Mod. Phys. Lett. A6 (1991) 1685-1692.

[95] E. Witten, "On string theory and black holes," Phys. Rev. D44 (1991) 314-324.

[96] S. Elitzur, A. Forge, and E. Rabinovici, "Some global aspects of string compactifications," Nucl. Phys. B359 (1991) 581-610.

[97] N. Itzhaki, J. M. Maldacena, J. Sonnenschein, and S. Yankielowicz, "Supergravity and the large $N$ limit of theories with sixteen supercharges," Phys. Rev. D58 (1998) 046004, hep-th/9802042.

[98] J. M. Maldacena and A. Strominger, "Semiclassical decay of near-extremal fivebranes," JHEP 12 (1997) 008, hep-th/9710014.

[99] J. Maharana and J. H. Schwarz, "Noncompact symmetries in string theory," Nucl. Phys. B390 (1993) 3-32, hep-th/9207016. 
[100] E. Bergshoeff, C. M. Hull, and T. Ortin, "Duality in the type II superstring effective action," Nucl. Phys. B451 (1995) 547-578, hep-th/9504081.

[101] C. G. Callan, S. S. Gubser, I. R. Klebanov, and A. A. Tseytlin, "Absorption of fixed scalars and the D-brane approach to black holes," Nucl. Phys. B489 (1997) 65-94, hep-th/9610172.

[102] N. D. Birrell and P. C. W. Davies, "Quantum fields in curved space,". Cambridge, United Kingdom: Univ. Pr. ( 1982) 340p.

[103] I. R. Klebanov and M. Krasnitz, "Fixed scalar greybody factors in five and four dimensions," Phys. Rev. D55 (1997) 3250-3254, hep-th/9612051.

[104] M. Krasnitz and I. R. Klebanov, "Testing effective string models of black holes with fixed scalars," Phys. Rev. D56 (1997) 2173-2179, hep-th/9703216.

[105] M. M. Taylor-Robinson, "Absorption of fixed scalars," hep-th/9704172.

[106] H. W. Lee, Y. S. Myung, and J. Y. Kim, "Absorption of fixed scalars in scattering off 5d black holes," Phys. Rev. D58 (1998) 104006, hep-th/9708099.

[107] S. S. Gubser, "Dynamics of D-brane black holes," hep-th/9908004.

[108] J. R. David, G. Mandal, and S. R. Wadia, "Absorption and Hawking radiation of minimal and fixed scalars, and AdS/CFT correspondence," Nucl. Phys. B544 (1999) 590-611, hep-th/9808168.

[109] J. Polchinski, "TASI lectures on D-branes," hep-th/9611050.

[110] M. R. Douglas, J. Polchinski, and A. Strominger, "Probing five-dimensional black holes with D-branes," JHEP 12 (1997) 003, hep-th/9703031.

[111] S. F. Hassan and S. R. Wadia, "Gauge theory description of D-brane black holes: Emergence of the effective SCFT and Hawking radiation," Nucl. Phys. B526 (1998) 311-333, hep-th/9712213.

[112] W. Taylor IV, "D-brane field theory on compact spaces," Phys. Lett. B394 (1997) 283-287, hep-th/9611042.

[113] E. Witten, "On the conformal field theory of the Higgs branch," JHEP 07 (1997) 003, hep-th/9707093.

[114] N. Seiberg and E. Witten, "The D1/D5 system and singular CFT," JHEP 04 (1999) 017, hep-th/9903224.

[115] J. L. Cardy, "Operator content of two-dimensional conformally invariant theories," Nucl. Phys. B270 (1986) 186-204.

[116] C. Vafa, "Instantons on D-branes," Nucl. Phys. B463 (1996) 435-442, hep-th/9512078. 
[117] M. R. Douglas, "Branes within branes," hep-th/9512077.

[118] E. Witten, "Sigma models and the ADHM construction of instantons," J. Geom. Phys. 15 (1995) 215-226, hep-th/9410052.

[119] R. Dijkgraaf, "Instanton strings and hyperKaehler geometry," Nucl. Phys. B543 (1999) 545-571, hep-th/9810210.

[120] C. Vafa, "Gas of D-branes and Hagedorn density of BPS states," Nucl. Phys. B463 (1996) 415-419, hep-th/9511088.

[121] A. Sen, "U-duality and intersecting D-branes," Phys. Rev. D53 (1996) 2874-2894, hep-th/9511026.

[122] M. Yu, "The unitary representations of the $N=4, S U(2)$ extended superconformal algebras," Nucl. Phys. B294 (1987) 890.

[123] O. Aharony, M. Berkooz, and E. Silverstein, "Non-local string theories on $A d S_{3} \times S^{3}$ and stable non- supersymmetric backgrounds," Phys. Rev. D65 (2002) 106007, hep-th/0112178.

[124] E. Witten, "Multi-trace operators, boundary conditions, and AdS/CFT correspondence," hep-th/0112258.

[125] L. J. Dixon, J. A. Harvey, C. Vafa, and E. Witten, "Strings on orbifolds," Nucl. Phys. B261 (1985) 678-686.

[126] L. J. Dixon, J. A. Harvey, C. Vafa, and E. Witten, "Strings on orbifolds. 2," Nucl. Phys. B274 (1986) 285-314.

[127] R. Dijkgraaf, G. W. Moore, E. Verlinde, and H. Verlinde, "Elliptic genera of symmetric products and second quantized strings," Commun. Math. Phys. 185 (1997) 197-209, hep-th/9608096.

[128] L. J. Dixon, D. Friedan, E. J. Martinec, and S. H. Shenker, "The conformal field theory of orbifolds," Nucl. Phys. B282 (1987) 13-73.

[129] M. Cvetic, "Effective lagrangian of the (blownup) oribifolds,". Superstrings, Unified Theories And Cosmology, ed. G. Furlan et. al,. (1987).

[130] S. F. Hassan and S. R. Wadia, "D-brane black holes: Large-N limit and the effective string description," Phys. Lett. B402 (1997) 43-52, hep-th/9703163.

[131] R. Dijkgraaf, E. Verlinde, and H. Verlinde, "5d black holes and matrix strings," Nucl. Phys. B506 (1997) 121-142, hep-th/9704018.

[132] E. Witten, "Constraints on supersymmetry breaking," Nucl. Phys. B202 (1982) 253. 
[133] H. J. Boonstra, B. Peeters, and K. Skenderis, "Duality and asymptotic geometries," Phys. Lett. B411 (1997) 59-67, hep-th/9706192.

[134] P. Claus et al., "Black holes and superconformal mechanics," Phys. Rev. Lett. 81 (1998) 4553-4556, hep-th/9804177.

[135] M. Gunaydin, G. Sierra, and P. K. Townsend, "The unitary supermultiplets of d $=3$ anti-de Sitter and d $=2$ conformal superalgebras," Nucl. Phys. B274 (1986) 429 .

[136] J. R. David, "Anti-de Sitter gravity associated with the supergroup $S U(1,1 \mid 2) \times S U(1,1 \mid 2), "$ Mod. Phys. Lett. A14 (1999) 1143-1148, hep-th/9904068.

[137] E. Gava, A. B. Hammou, J. F. Morales, and K. S. Narain, "D1/D5 systems in $\mathcal{N}=4$ string theories," Nucl. Phys. B605 (2001) 17-63, hep-th/0012118.

[138] E. Gava, A. B. Hammou, J. F. Morales, and K. S. Narain, "AdS/CFT correspondence and D1/D5 systems in theories with 16 supercharges," JHEP 03 (2001) 035, hep-th/0102043.

[139] A. Giveon, D. Kutasov, and N. Seiberg, "Comments on string theory on $A d S_{3}$," Adv. Theor. Math. Phys. 2 (1998) 733-780, hep-th/9806194.

[140] E. Witten, "AdS/CFT correspondence and topological field theory," JHEP 12 (1998) 012, hep-th/9812012.

[141] J. R. David, G. Mandal, and S. R. Wadia, "D1/D5 moduli in SCFT and gauge theory, and Hawking radiation," Nucl. Phys. B564 (2000) 103-127, hep-th/9907075.

[142] F. Larsen and E. J. Martinec, "U(1) charges and moduli in the D1-D5 system," JHEP 06 (1999) 019, hep-th/9905064.

[143] C. Vafa, "Quantum symmetries of string vacua," Mod. Phys. Lett. A4 (1989) 1615 .

[144] A. Mikhailov, "D1D5 system and noncommutative geometry," Nucl. Phys. B584 (2000) 545-588, hep-th/9910126.

[145] O. Aharony and M. Berkooz, "IR dynamics of $d=2, \mathcal{N}=(4,4)$ gauge theories and DLCQ of 'little string theories'," JHEP 10 (1999) 030, hep-th/9909101.

[146] P. S. Aspinwall, "Enhanced gauge symmetries and K3 surfaces," Phys. Lett. B357 (1995) 329-334, hep-th/9507012.

[147] E. Witten, "Some comments on string dynamics," hep-th/9507121.

[148] C. P. Bachas, "Lectures on D-branes," hep-th/9806199. 
[149] G. Arutyunov, A. Pankiewicz, and S. Theisen, "Cubic couplings in D =6 N = 4b supergravity on $A d S_{3} \times S^{3}$," Phys. Rev. D63 (2001) 044024, hep-th/0007061.

[150] D. Z. Freedman, S. D. Mathur, A. Matusis, and L. Rastelli, "Correlation functions in the $\mathrm{CFT}_{d} / \mathrm{AdS}_{d+1}$ correspondence," Nucl. Phys. B546 (1999) 96-118, hep-th/9804058.

[151] O. Lunin and S. D. Mathur, "Correlation functions for $M^{N} / S^{N}$ orbifolds," Commun. Math. Phys. 219 (2001) 399-442, hep-th/0006196.

[152] O. Lunin and S. D. Mathur, "Three-point functions for $M^{N} / S^{N}$ orbifolds with n = 4 supersymmetry," Commun. Math. Phys. 227 (2002) 385-419, hep-th/0103169.

[153] G. E. Arutyunov and S. A. Frolov, "Virasoro amplitude from the $S^{N} R^{24}$ orbifold sigma model," Theor. Math. Phys. 114 (1998) 43-66, hep-th/9708129.

[154] G. E. Arutyunov and S. A. Frolov, "Four graviton scattering amplitude from $S^{N} R^{8}$ supersymmetric orbifold sigma model," Nucl. Phys. B524 (1998) 159-206, hep-th/9712061.

[155] S. S. Gubser, "Absorption of photons and fermions by black holes in four dimensions," Phys. Rev. D56 (1997) 7854-7868, hep-th/9706100.

[156] D. Birmingham, I. Sachs, and S. Sen, "Three-dimensional black holes and string theory," Phys. Lett. B413 (1997) 281-286, hep-th/9707188.

[157] H. J. W. Muller-Kirsten, N. Ohta, and J.-G. Zhou, "Ad $S_{3} / C F T$ correspondence, poincare vacuum state and greybody factors in BTZ black holes," Phys. Lett. B445 (1999) 287-295, hep-th/9809193.

[158] N. Ohta and J.-G. Zhou, "Thermalization of poincare vacuum state and fermion emission from $A d S_{3}$ black holes in bulk-boundary correspondence," JHEP 12 (1998) 023, hep-th/9811057.

[159] H. W. Lee and Y. S. Myung, "Holographic connection between the BTZ black hole and 5d black hole," Phys. Rev. D58 (1998) 104013, hep-th/9804095.

[160] H. W. Lee and Y. S. Myung, "Scattering from an $A d S_{3}$ bubble and an exact Ad $S_{3}, "$ Phys. Rev. D61 (2000) 024031, hep-th/9903054.

[161] E. Teo, "Black hole absorption cross-sections and the anti-de Sitter-conformal field theory correspondence," Phys. Lett. B436 (1998) 269-274, hep-th/9805014.

[162] I. R. Klebanov, A. Rajaraman, and A. A. Tseytlin, "Intermediate scalars and the effective string model of black holes," Nucl. Phys. B503 (1997) 157-176, hep-th/9704112.

[163] S. Cecotti, " $N=2$ Landau-Ginzburg versus Calabi-Yau sigma models: Nonperturbative aspects," Int. J. Mod. Phys. A6 (1991) 1749-1814. 
[164] E. Witten, "Dynamical breaking of supersymmetry," Nucl. Phys. B188 (1981) 513.

[165] J. M. Maldacena, G. W. Moore, and A. Strominger, "Counting BPS black holes in toroidal type II string theory," hep-th/9903163.

[166] S. R. Das, "The effectiveness of D-branes in the description of near-extremal black holes," Phys. Rev. D56 (1997) 3582-3590, hep-th/9703146.

[167] A. B. Zamolodchikov, "Irreversibility' of the flux of the renormalization group in a 2-d field theory," JETP Lett. 43 (1986) 730-732.

[168] I. Pesando, "The GS type IIB superstring action on $A d S_{3} \times S^{3} \times T^{4}$," JHEP 02 (1999) 007, hep-th/9809145.

[169] J. Rahmfeld and A. Rajaraman, "The GS string action on $A d S_{3} \times S^{3}$ with Ramond-Ramond charge," Phys. Rev. D60 (1999) 064014, hep-th/9809164.

[170] J. Park and S.-J. Rey, "Green-Schwarz superstring on $A d S_{3} \times S^{3}$," JHEP 01 (1999) 001, hep-th/9812062.

[171] M. Yu and B. Zhang, "Light-cone gauge quantization of string theories on $A d S_{3}$ space," Nucl. Phys. B551 (1999) 425-449, hep-th/9812216.

[172] N. Berkovits, C. Vafa, and E. Witten, "Conformal field theory of AdS background with Ramond-Ramond flux," JHEP 03 (1999) 018, hep-th/9902098.

[173] L. Dolan and E. Witten, "Vertex operators for AdS(3) background with Ramond-Ramond flux," JHEP 11 (1999) 003, hep-th/9910205.

[174] J. Maldacena and H. Ooguri, "Strings in $A d S_{3}$ and $S L(2, R)$ WZW model. I," $J$. Math. Phys. 42 (2001) 2929-2960, hep-th/0001053.

[175] J. Maldacena, H. Ooguri, and J. Son, "Strings in $A d S_{3}$ and the $S L(2, R)$ WZW model. II: Euclidean black hole," J. Math. Phys. 42 (2001) 2961-2977, hep-th/0005183.

[176] J. Maldacena and H. Ooguri, "Strings in $A d S_{3}$ and the $S L(2, R)$ WZW model. III: Correlation functions," hep-th/0111180.

[177] P. Lee, H. Ooguri, and J. Park, "Boundary states for $A d S_{2}$ branes in $A d S_{3}$," hep-th/0112188.

[178] B. Ponsot, V. Schomerus, and J. Teschner, "Branes in the Euclidean $A d S_{3}$," hep-th/0112198.

[179] L. J. Dixon, M. E. Peskin, and J. Lykken, " $N=2$ superconformal symmetry and SO $(2,1)$ current algebra," Nucl. Phys. B325 (1989) 329-355. 
[180] P. Breitenlohner and D. Z. Freedman, "Positive energy in anti-de Sitter backgrounds and gauged extended supergravity," Phys. Lett. B115 (1982) 197.

[181] J. M. Evans, M. R. Gaberdiel, and M. J. Perry, "The no-ghost theorem for $A d S_{3}$ and the stringy exclusion principle," Nucl. Phys. B535 (1998) 152-170, hep-th/9806024.

[182] Y. Satoh, "On string theory in $A d S_{3}$ backgrounds," hep-th/0005169.

[183] M. Wakimoto, "Fock representations of the affine lie algebra A1(1)," Commun. Math. Phys. 104 (1986) 605-609.

[184] C. G. Callan, J. A. Harvey, and A. Strominger, "Supersymmetric string solitons," hep-th/9112030.

[185] D. J. Gross, M. J. Perry, and L. G. Yaffe, "Instability of flat space at finite temperature," Phys. Rev. D25 (1982) 330-355.

[186] S. W. Hawking and D. N. Page, "Thermodynamics of black holes in anti-de Sitter space," Commun. Math. Phys. 87 (1983) 577.

[187] M. Nishimura and Y. Tanii, "Super weyl anomalies in the AdS/CFT correspondence," Int. J. Mod. Phys. A14 (1999) 3731-3744, hep-th/9904010.

[188] H. Nicolai and H. Samtleben, "N $=8$ matter coupled $A d S_{3}$ supergravities," Phys. Lett. B514 (2001) 165-172, hep-th/0106153.

[189] E. Witten, "Anti-de Sitter space, thermal phase transition, and confinement in gauge theories," Adv. Theor. Math. Phys. 2 (1998) 505-532, hep-th/9803131.

[190] J. R. David, G. Mandal, S. Vaidya, and S. R. Wadia, "Point mass geometries, spectral flow and $\mathrm{AdS}_{3}-\mathrm{CFT}_{2}$ correspondence," Nucl. Phys. B564 (2000) 128-141, hep-th/9906112.

[191] R. Dijkgraaf, J. Maldacena, G. W. Moore, and E. Verlinde, "A black hole farey tail," hep-th/0005003.

[192] S. Deser, R. Jackiw, and G. 't Hooft, "Three-dimensional Einstein gravity: Dynamics of flat space," Ann. Phys. 152 (1984) 220.

[193] S. Deser and R. Jackiw, "Three-dimensional cosmological gravity: Dynamics of constant curvature," Annals Phys. 153 (1984) 405-416.

[194] J. M. Izquierdo and P. K. Townsend, "Supersymmetric space-times in $(2+1)$ ads supergravity models," Class. Quant. Grav. 12 (1995) 895-924, gr-qc/9501018.

[195] A. Achucarro and P. K. Townsend, "Extended supergravities in d $=(2+1)$ as Chern-Simons theories," Phys. Lett. B229 (1989) 383. 
[196] V. Balasubramanian, J. de Boer, E. Keski-Vakkuri, and S. F. Ross, "Supersymmetric conical defects: Towards a string theoretic description of black hole formation," Phys. Rev. D64 (2001) 064011, hep-th/0011217.

[197] J. Maldacena and L. Maoz, "De-singularization by rotation," hep-th/0012025.

[198] E. J. Martinec and W. McElgin, "String theory on AdS orbifolds," hep-th/0106171.

[199] H.-J. Matschull, "Black hole creation in 2+1-dimensions," Class. Quant. Grav. 16 (1999) 1069-1095, gr-qc/9809087.

[200] M. W. Choptuik, "Universality and scaling in gravitational collapse of a massless scalar field," Phys. Rev. Lett. 70 (1993) 9-12.

[201] Y. Peleg and A. R. Steif, "Phase transition for gravitationally collapsing dust shells in (2+1)-dimensions," Phys. Rev. D51 (1995) 3992-3996, gr-qc/9412023.

[202] D. Birmingham, "Choptuik scaling and quasinormal modes in the AdS/CFT correspondence," Phys. Rev. D64 (2001) 064024, hep-th/0101194.

[203] U. H. Danielsson, E. Keski-Vakkuri, and M. Kruczenski, "Spherically collapsing matter in ads, holography, and shellons," Nucl. Phys. B563 (1999) 279-292, hep-th/9905227.

[204] U. H. Danielsson, E. Keski-Vakkuri, and M. Kruczenski, "Black hole formation in ads and thermalization on the boundary," JHEP 02 (2000) 039, hep-th/9912209.

[205] S. B. Giddings and A. Nudelman, "Gravitational collapse and its boundary description in ads," JHEP 02 (2002) 003, hep-th/0112099.

[206] N. Dorey, T. J. Hollowood, V. V. Khoze, M. P. Mattis, and S. Vandoren, "Multi-instantons and Maldacena's conjecture," JHEP 06 (1999) 023, hep-th/9810243.

[207] M. Blau, K. S. Narain, and G. Thompson, "Instantons, the information metric, and the AdS/CFT correspondence," hep-th/0108122.

[208] S. R. Das, S. Naik, and S. R. Wadia, "Quantization of the Liouville mode and string theory," Mod. Phys. Lett. A4 (1989) 1033.

[209] A. Dhar, T. Jayaraman, K. S. Narain, and S. R. Wadia, "The role of quantized two-dimensional gravity in string theory," Mod. Phys. Lett. A5 (1990) 863.

[210] S. R. Das, A. Dhar, and S. R. Wadia, "Critical behavior in two-dimensional quantum gravity and equations of motion of the string," Mod. Phys. Lett. A5 (1990) 799.

[211] A. Dhar and S. R. Wadia, "Noncritical strings, RG flows and holography," Nucl. Phys. B590 (2000) 261-272, hep-th/0006043. 
[212] K. Krasnov, "3D gravity, point particles and Liouville theory," Class. Quant. Grav. 18 (2001) 1291-1304, hep-th/0008253.

[213] K. Krasnov, "Lambda $<0$ quantum gravity in 2+1 dimensions. I: Quantum states and stringy S-matrix," hep-th/0112164.

[214] K. Krasnov, "Lambda $<0$ quantum gravity in 2+1 dimensions. II: Black hole creation by point particles," hep-th/0202117.

[215] T. Banks, M. R. Douglas, G. T. Horowitz, and E. J. Martinec, "AdS dynamics from conformal field theory," hep-th/9808016.

[216] V. Balasubramanian, S. B. Giddings, and A. E. Lawrence, "What do CFTs tell us about anti-de Sitter spacetimes?," JHEP 03 (1999) 001, hep-th/9902052.

[217] J. D. Brown and M. Henneaux, "Central charges in the canonical realization of asymptotic symmetries: An example from three-dimensional gravity," Commun. Math. Phys. 104 (1986) 207-226.

[218] R. Dijkgraaf, E. Verlinde, and H. Verlinde, "5d black holes and matrix strings," Nucl. Phys. B506 (1997) 121-142, hep-th/9704018.

[219] D. Kabat, G. Lifschytz, and D. A. Lowe, "Black hole entropy from non-perturbative gauge theory," Phys. Rev. D64 (2001) 124015, hep-th/0105171.

[220] S. A. Abel, J. L. F. Barbon, I. I. Kogan, and E. Rabinovici, "Some thermodynamical aspects of string theory," hep-th/9911004.

[221] J. L. F. Barbon and E. Rabinovici, "Closed-string tachyons and the hagedorn transition in AdS space," hep-th/0112173.

[222] A. Dabholkar, "Tachyon condensation and black hole entropy," Phys. Rev. Lett. 88 (2002) 091301, hep-th/0111004.

[223] A. Adams, J. Polchinski, and E. Silverstein, "Don't panic! closed string tachyons in ALE space-times," JHEP 10 (2001) 029, hep-th/0108075.

[224] D. Berenstein, J. Maldacena, and H. Nastase, "Strings in flat space and pp waves from $N=4$ super yang mills," hep-th/0202021.

[225] L. A. P. Zayas and J. Sonnenschein, "On Penrose limits and gauge theories," hep-th/0202186.

[226] V. Kazakov, I. K. Kostov, and D. Kutasov, "A matrix model for the two-dimensional black hole," Nucl. Phys. B622 (2002) 141-188, hep-th/0101011.

[227] J. M. Maldacena, "Statistical entropy of near extremal five-branes," Nucl. Phys. B477 (1996) 168-174, hep-th/9605016. 
[228] A. Hanany, N. Prezas, and J. Troost, "The partition function of the two-dimensional black hole conformal field theory," hep-th/0202129.

[229] A. Sen, "Rotating charged black hole solution in heterotic string theory," Phys. Rev. Lett. 69 (1992) 1006-1009, hep-th/9204046.

[230] G. T. Horowitz, "The dark side of string theory: Black holes and black strings," hep-th/9210119.

[231] J. G. Russo and A. A. Tseytlin, "Waves, boosted branes and BPS states in M-theory," Nucl. Phys. B490 (1997) 121-144, hep-th/9611047.

[232] M. Banados, M. Henneaux, C. Teitelboim, and J. Zanelli, "Geometry of the (2+1) black hole," Phys. Rev. D48 (1993) 1506-1525, gr-qc/9302012. 